\title{
Nematic Liquid Crystals and Nematic Colloids in Microfluidic Environment
}

\section{Dissertation}

zur Erlangung des mathematisch-naturwissenschaftlichen Doktorgrades

"Doctor rerum naturalium"

der Georg-August-Universität Göttingen

im Promotionsprogramm ProPhys

der Georg-August University School of Science (GAUSS)

vorgelegt von

\section{Anupam Sengupta}

aus Burnpur, Indien

Göttingen, 2012 


\section{Betreuungsausschuss}

Betreuer : Prof. Dr. Jörg Enderlein, Drittes Physikalisches Institut, Universität Göttingen

Betreuer : Prof. Dr. Stephan Herminghaus, Dynamik komplexer Fluide, MPIDS ${ }^{\dagger}$, Göttingen

\section{Mitglieder der Prüfungskommission}

Referent： Prof. Dr. Jörg Enderlein, Drittes Physikalisches Institut, Universität Göttingen

Koreferent : Prof. Dr. Stephan Herminghaus, Dynamik komplexer Fluide, MPIDS ${ }^{\dagger}$, Göttingen

Koreferent : Prof. Dr. Pawel Pieranski, Laboratoire de Physique des Solides, University Paris-South, Paris

\section{Weitere Mitglieder der Prüfungskommission}

Prof. Dr. Tim Salditt, Institut für Röntgenphysik, Universität Göttingen

Dr. Christian Bahr, Dynamik komplexer Fluide, MPIDS ${ }^{\dagger}$, Göttingen

Dr. Simone Techert, Strukturdynamik (bio)chemischer Systeme, MPIBPC ${ }^{\ddagger}$, Göttingen

Prof. Dr. Sarah Köster, Institut für Röntgenphysik, Universität Göttingen

Tag der mündlichen Prüfung : 18.12.2012

$\dagger$ Max-Planck-Institut für Dynamik und Selbstorganisation

¥ Max-Planck-Institut für Biophysikalische Chemie 
Where the mind is without fear and the head is held high;

Where knowledge is free;

Where the world has not been broken up into fragments by narrow domestic walls;

Where words come out from the depth of truth;

Where tireless striving stretches its arms towards perfection;

Where the clear stream of reason has not lost its way into the dreary desert sand of dead habit;

Where the mind is led forward by thee into ever-widening thought and action Into that heaven of freedom, my Father, let my country awake.

Rabindranath Tagore (1861 - 1941) 

To my parents, my brother, and all the sacrifices they made for me 



\section{Abstract}

The doctoral thesis presented here is one of the first systematic attempts to unravel the wonderful world of liquid crystals within microfluidic confinements, typically channels with dimensions of tens of micrometers. The present work is based on experiments with a roomtemperature nematic liquid crystal, 5CB, and its colloidal dispersions within microfluidic devices of rectangular cross-section, fabricated using standard techniques of soft lithography. To begin with, a combination of physical and chemical methods was employed to create well defined boundary conditions for investigating the flow experiments. The walls of the microchannels were functionalized to induce different kinds of surface anchoring of the 5CB molecules: degenerate planar, uniform planar, and homeotropic surface anchoring. Channels possessing composite anchoring conditions (hybrid) were additionally fabricated, e. g. homeotropic and uniform planar anchoring within the same channel. On filling the microchannels with 5CB in the isotropic phase, different equilibrium configurations of the nematic director resulted, as the sample cooled down to nematic phase. For a given surface anchoring, the equilibrium director configuration varied also with the channel aspect ratio. The static director field within the channel registered the initial conditions for the flow experiments. The static and 
dynamic experiments have been analyzed using a combination of polarization, and confocal fluorescence microscopy techniques, along with particle tracking method for measuring the flow speeds. Additionally, dual-focus fluorescence correlation spectroscopy is introduced as a generic velocimetry tool for liquid crystal flows.

The flow of nematic liquid crystals is inherently complex due to the coupling between the flow and the nematic director. The presence of the four confining walls and the nature of surface anchoring on them complicate the flow-director interactions further. In microchannels possessing degenerate planar anchoring, four different flow-induced defect textures were identified with increasing Ericksen number: $\pi$-walls, disclination lines pinned to the channel walls, disclination lines with one pinned and one freely suspended end, and disclination loops freely flowing in a chaotic manner. However, such textures and sequence of defects were not observed for flows within channels with homogeneous anchoring.

Using experiments and numerical modeling the flow-director coupling was investigated within homeotropic microchannels. Complex non-Poiseuille multi-stream flow profiles emerged which provided a direct route to controlled shaping of the flow profile in a microfluidic channel. The dynamics have been characterized by the de Gennes characteristic shear-flow lengths $e_{1}$ and $e_{2}$ which, together with the channel's aspect ratio $w / d$, control the relative stability of the flow regimes. Additionally, by applying a local temperature gradient across the channel, the nematic flow could be steered in the transverse direction via mechanisms of viscosity anisotropy. The flow-director coupling was quantified through optical birefringence and in situ velocity measurements within a diverging microchannel. When a cylindrical obstacle 
was placed in the flow path, a reversible sequence of topological defects originated at the obstacle. The appearance of the topological structures has been analyzed on the basis of the flow-director interactions at different flow speeds. Using the dual-focus fluorescence correlation method, the velocity distribution within the defect structure was experimentally assessed.

The flow of nematic 5CB within a microchannel with hybrid surface anchoring (combination of surfaces having uniform planar and homeotropic anchoring) generated and stabilized a topological defect line along the entire length of the microchannel. Colloid particles and small water droplets, the 'working horses' of common-style droplet-based microfluidics, were trapped at the disclination lines and consequently followed them through the microfluidic device. The topological defect line was utilized as a 'soft rail' whose position was controlled through easily accessible experimental parameters. Controlled threading of a defect line at a channel bifurcation and in situ switching of the defect guidance demonstrate the high potential of this technique, especially for the transport of a wide range of microfluidic cargo. The topological soft rail introduces a unique platform for targeted delivery of single particles, droplets, or clusters of such entities, paving the way to flexible micro-cargo concepts in microfluidic settings.

Colloidal particles transported through the nematic matrix were further utilized to extract the information about the flow-induced local director field. The dependence of the particle orientation flowing through the ordered $5 \mathrm{CB}$ has been proposed as a route to stereo-selective transport of colloidal inclusions (with shape anisotropy) under appropriate boundary conditions. In addition, the interplay between the viscous and elastic interactions present in such 
systems has been utilized to derive the particle-disclination trapping force. A number of new questions evolved during the course of the research work. Suggestive experiments to address those questions, and a perspective view on the research of liquid crystal based microfluidics, are presented in the concluding parts of the dissertation. 


\section{Kurzzusammenfassung}

Die vorliegende Doktorarbeit beschreibt eine systematische Untersuchung des Verhaltens von nematischen Flüssigkristallen in Mikrofluidik-Kanälen mit Abmessungen im Bereich von 10 bis $100 \mu \mathrm{m}$. Die experimentellen Arbeiten wurden mit der Flüssigkristallverbindung 5CB, die bei Raumtemperatur in der nematischen Phase vorliegt, sowie mit Dispersionen von Kolloidteilchen in 5CB durchgeführt; die Mikrofluidik-Kanäle wiesen einen rechteckigen Querschnitt auf und wurden mit Standardtechniken der "weichen" Lithographie hergestellt. Zunächst wurde eine Kombination von physikalischen und chemischen Methoden zur Erzeugung einer definierten Oberflächenverankerung des nematischen Direktors entwickelt. Die Oberflächen der Kanalwände wurden so funktionalisiert, dass entweder ein entarteter planarer, ein uniformer planarer oder ein homöotroper Verankerungszustand erzeugt wurde. Auch Kanäle mit einer hybriden Verankerung, bei der z. B. drei Wände eine homöotrope und eine Wand eine planare Verankerung aufwiesen, konnten hergestellt werden. Die Art der Oberflächenverankerung sowie das Aspektverhältnis der Kanäle bestimmten das statische Direktorfeld, das sich nach dem Füllen der Kanäle mit dem nematischen Flüssigkristall ausbildete und das den Anfangszustand der nachfolgenden Untersuchungen des Fließverhaltens 
definierte. Polarisationsmikroskopie und konfokale Fluoreszenzmikroskopie wurden eingesetzt, um das statische und dynamische Direktorfeld zu bestimmen. Die lokale Strömungsgeschwindigkeit wurde mit Partikel-Tracking-Methoden sowie mit Hilfe der Dual-Fokus-Fluoreszenzkorrelationsspektroskopie gemessen.

Das Fließverhalten von nematischen Flüssigkristallen besitzt eine inhärente Komplexität aufgrund der Kopplung zwischen der Strömung, der Orientierung des Direktors und der Viskosität. Die Begrenzung des Volumens durch die Mikrofluidik-Kanäle sowie der Einfluss der Oberflächenverankerung sind weitere wesentliche Faktoren, die das Fließverhalten beeinflussen. Der generische Verankerungszustand, der in Mikrofluidik-Kanälen ohne weitere Funktionalisierung erhalten wird, ist entartet planar (der Direktor orientiert sich parallel zur Kanalwand, wobei keine bevorzugte Richtung der Orientierung in der Wandebene besteht). In Mikrofluidik-Kanälen mit entartet planarer Oberflächenverankerung wurden mit zunehmender Ericksen-Zahl (d. h. mit zunehmender Strömungsgeschwindigkeit und/oder zunehmendem hydraulischen Durchmesser) vier Bereiche beobachtet, die sich durch ihre Texturen und Defektstrukturen unterscheiden: $\pi$-Wände; Disklinationslinien, deren beide Enden an den Kanalwänden haften; Disklinationslinien mit einem freien und einem haftenden Ende; in sich geschlossene Disklinationsschleifen, die frei in der Strömung treiben und sich dabei irregulär und chaotisch bewegen. Mikrofluidik-Kanäle mit homöotroper oder uniform planarer Verankerung weisen strömungsinduzierte Zustände auf, die in wesentlich geringerem Ausmaß durch Defektstrukturen charakterisiert sind.

In Mikrofluidik-Kanälen mit homöotroper Oberflächenverankerung (der Direktor orien- 
tiert sich senkrecht zur Kanalwand) wurde die Kopplung zwischen der Strömung und der Orientierung des Direktors experimentell untersucht und mit Ergebnisen von numerischen Simulationen verglichen. Mit zunehmender Strömungsgeschwindigkeit wurden verschiedene Bereiche mit teilweise komplexen Stömungsprofilen beobachtet, die nicht einem einfachen Poiseuille-Profil entsprechen. Die Stabilität dieser Bereiche wird durch die von de Gennes beschriebenen Scherströmungslängen $e_{1}$ und $e_{2}$ sowie dem Aspektverhältnis der Kanäle bestimmt. Es wurde gezeigt, dass die Gegenwart eines Temperaturgradienten senkrecht zur Kanallängsachse einen erheblichen Einfluss auf die Form des Strömungsprofils ausübt. Mithilfe eines Mikrofluidik-Kanals mit divergierender Breite wurde eine quantitative Anwendung zur Messung der Strömungsgeschwindigkeit in situ demonstriert, die auf der Direktorreorientierung und der dadurch induzierten Doppelbrechung im fließenden nematischen Flüssigkristall beruht. Weiterhin wurden die Effekte untersucht, die die Platzierung eines zylindrischen Hindernisses im Mikrofluidik-Kanal hervorruft. Dabei wurde in Abhängigkeit der Strömungsgeschwindigkeit eine reversible Abfolge verschiedener Defektstrukturen beobachtet und jeweils mit Hilfe der Dual-Fokus-Fluoreszenzkorrelationspektroskopie das Strömungsgeschwindigkeitsprofil bestimmt.

Für Mikrofluidik-Kanäle mit hybriden Verankerungsbedingungen (homöotrop auf drei Wänden, uniform planar auf der vierten Wand) wurde gezeigt, dass in der fließenden nematischen Phase eine durchgehende Disklinationslinie entlang der Kanallängsachse im gesamten Mikrofluidik-Kanal erzeugt und stabilisiert werden kann. Es wurde demonstriert, dass die Disklinationslinie als "weiche Schiene" für den gelenkten Transport von Kolloidteilchen und 
viii

wässrigen Tröpfchen, die von der Disklinationslinie "eingefangen" wurden, genutzt werden kann. Weiterhin wurde gezeigt, dass die Richtung der uniformen planaren Verankerung an der vierten Kanalwand dazu genutzt werden, kann die Lage der Disklinationslinie im Kanal sowie ihr Verhalten an einer Kanal-Bifurkation zu steuern. Das hier beschriebene Konzept eröffnet neue Möglichkeiten für den kontrollierten Transport von Teilchen und Tröpfchen in der Mikrofluidik.

Kolloidpartikel, die mit der nematischen Matrix transportiert wurden, konnten auch dazu genutzt werden, Informationen über die lokale Orientierung des Direktors in der fließenden nematischen Phase zu erhalten. Die Kopplung zwischen Strömung und Direktor ermöglichte außerdem für formanisotrope Kolloidteilchen, oder für lineare Aggregate von Kolloidteilchen, eine Auswahl, ob die Teilchen bzw. Aggregate mit ihrer Längsachse parallel oder senkrecht zur Strömungsrichtung transportiert werden. Die anziehende Wechselwirkung zwischen den Kolloidteilchen und den Disklinationslinien konnte im Mikrofluidik-Kanal bestimmt werden. Die Dissertation schließt mit einem Ausblick auf die Mikrofluidik mit thermotropen Flüssigkristallen und mit Vorschlägen für weitere Experimente, die Fragestellungen betreffen, die sich im Verlauf der vorliegenden Arbeit ergeben haben. 


\section{Contents}

Abstract ............................. i

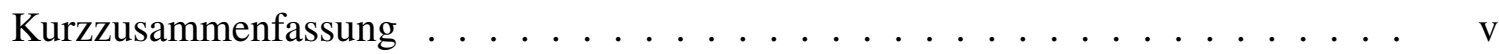

Contents ............................ . . . . . . .

List of Figures $\ldots \ldots \ldots \ldots \ldots \ldots$. . . . . . . . . . . . . . . . . . . . . .

List of Tables . . . . . . . . . . . . . . . . . . . xvii

1 Introduction 1

1.1 Liquid crystals: Complex anisotropic fluids $\ldots \ldots \ldots \ldots$. . . . . . . . 1

1.2 Microfluidics . . . . . . . . . . . . . . . . . . 2

1.3 Motivation . . . . . . . . . . . . . . . . . . 2

1.4 Thesis outline $\ldots \ldots \ldots \ldots \ldots \ldots \ldots \ldots$

2 Liquid crystal theory 5

2.1 Liquid crystal mesophases $\ldots \ldots \ldots \ldots \ldots$

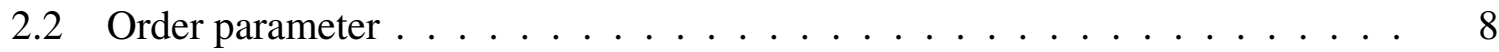

2.3 Landau-de Gennes theory . . . . . . . . . . . . . . . . . . . . . . 10

2.3 .1 Phase transition . . . . . . . . . . . . . . . . . . . . 10

2.3 .2 Nematoelasticity . . . . . . . . . . . . . . . 12

2.3 .3 Landau-de Gennes free energy . . . . . . . . . . . . . . . . . . 14

2.4 Surface anchoring . . . . . . . . . . . . . . . . . . . . . 15

2.5 Anisotropy in liquid crystals $\ldots \ldots \ldots \ldots \ldots \ldots$

2.5 .1 Optical anisotropy $\ldots \ldots \ldots \ldots$. . . . . . . . . . . . 17

2.5 .2 Viscosity . . . . . . . . . . . . . . . . . . . . . . . . . 19

2.6 Topological defects $\ldots \ldots \ldots \ldots \ldots \ldots$

2.7 Flow of nematic liquid crystals: Nematodynamics . . . . . . . . . . . . 25

2.7 .1 Ericksen-Leslie theory of nematodynamics . . . . . . . . . 26

2.7 .2 Poiseuille flow of nematic liquid crystals $\ldots \ldots \ldots \ldots$ 
2.7 .3 Topological defects in flow $\ldots \ldots \ldots \ldots \ldots \ldots$

2.8 Nematic colloids . . . . . . . . . . . . . . . . . . . . . . . 34

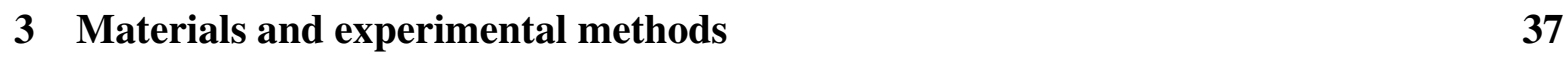

$3.1 \quad$ Nematic liquid crystal $\ldots \ldots \ldots \ldots . \ldots \ldots$

3.2 Preparation of the nematic colloids . . . . . . . . . . . . . . 40

3.3 Microfluidic confinement and flow set-up . . . . . . . . . . . . . 41

3.3.1 Fabrication of microfluidic devices . . . . . . . . . . . . . . 41

3.3 .2 Flow setup $\ldots \ldots \ldots \ldots \ldots \ldots$

3.3 .3 Functionalization of microfluidic devices . . . . . . . . . . . 44

3.4 Characterization techniques . . . . . . . . . . . . . . . . . . . . . . . . . 44

$3.4 .1 \quad$ Polarization optical microscopy $\ldots \ldots \ldots \ldots \ldots$. . . . . . 44

$3.4 .2 \quad$ Fluorescence confocal polarization microscopy . . . . . . . . . . 47

3.4 .3 Particle tracking method $\ldots \ldots \ldots \ldots \ldots$

3.4 .4 Dual-focus fluorescence correlation spectroscopy . . . . . . . . . 50

4 Functionalization of microfluidic devices $\quad 53$

4.1 Non-trivial aspects of microchannel functionalization . . . . . . . . . . . 53

4.2 Anchoring characterization of functionalized substrates . . . . . . . . . 55

4.3 Microchannel functionalization and characterization of surface anchoring . . 60

$4.3 .1 \quad$ Degenerate planar anchoring . . . . . . . . . . . . . 60

4.3 .2 Uniform planar anchoring $\ldots \ldots \ldots \ldots \ldots \ldots$

4.3 .3 Homeotropic anchoring $\ldots \ldots \ldots \ldots \ldots$

4.3 .4 Hybrid anchoring . . . . . . . . . . . . . . . 66

$5 \quad$ Nematic liquid crystals confined within a microfluidic device: Static case

$5.1 \quad$ Liquid crystals in confinements . . . . . . . . . . . . . . . . . . . 69

5.2 Microchannels with planar surface anchoring $\ldots \ldots \ldots \ldots 70$

5.3 Microchannels with homeotropic surface anchoring . . . . . . . . . . 72

5.4 Homeotropic microchannel with cylindrical micro-pillar . . . . . . . . . 77

5.5 Microchannels with hybrid anchoring $\ldots \ldots \ldots \ldots . \ldots . \ldots 81$

$6 \quad$ Flow of nematic liquid crystals in a microfluidic environment 85

$6.1 \quad$ Elastic, surface and viscous interactions on a microfluidic platform . . . . . 85

6.2 Nematic flow due to pressure gradient . . . . . . . . . . . . . . 87

6.3 Nematic flow in a degenerate planar microchannel . . . . . . . . . . . . 91 
6.4 Nematic flow in a homeotropic microchannel . . . . . . . . . . . . . . . . 101

6.4 .1 Tunable flow shaping . . . . . . . . . . . . . . . . . . . 102

6.4 .2 Application of a transverse temperature gradient . . . . . . . . 108

6.4 .3 Opto-fluidic velocimetry in a diverging channel . . . . . . . . . . . 109

6.5 Nematic flow past a cylindrical micro-pillar . . . . . . . . . . . . . 116

$6.5 .1 \quad$ Semi-integer defect loop . . . . . . . . . . . . . . . . . . 120

6.5 .2 Morphology of the wall defect . . . . . . . . . . . . . . . . 123

6.5 .3 Dynamics of the wall defect $\ldots \ldots \ldots \ldots . \ldots . \ldots 125$

6.5 .4 Flow reversal: Bloch wall to Néel wall transformation . . . . . . . . 127

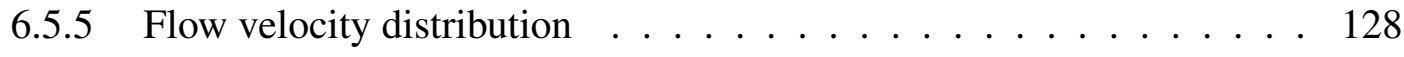

6.6 Nematic flow in a hybrid microchannel . . . . . . . . . . . . . . . . . . 131

6.6 .1 Creation-cum-stabilization of the topological soft rail . . . . . . . . . 132

6.6 .2 Navigating the topological defect at a flow bifurcation . . . . . . . 135

6.7 Transition to the chaotic regime $\ldots \ldots \ldots \ldots \ldots$

7 Nematic colloids in microfluidic confinement 143

7.1 Guided transport of microfluidic cargo on soft rails . . . . . . . . . . . 143

7.2 Measurement of the particle-disclination interaction . . . . . . . . . . . 147

7.3 Director field mapping . . . . . . . . . . . . . . . . . . . . . 148

8 Ongoing research 151

$\begin{array}{lll}9 & \text { Conclusion } & 153\end{array}$

\begin{tabular}{ll}
\hline References & 157
\end{tabular}

\begin{tabular}{lr}
\hline Acknowledgements & 171
\end{tabular} 



\section{List of Figures}

$2.1 \quad$ Shape-anisotropy and liquid crystal phases . . . . . . . . . . . . . 6

2.2 Generic shape of the distribution function in an uniaxial nematic . . . . . . . 9

2.3 Variation of free energy density and order parameter. . . . . . . . . . 12

2.4 Elastic deformations in nematic liquid crystals . . . . . . . . . . . . . . 13

2.5 Anchoring of nematic liquid crystals on a surface $\ldots \ldots \ldots \ldots$

2.6 Anchoring angles $\ldots \ldots \ldots \ldots \ldots \ldots$

2.7 Optical anisotropy $\ldots \ldots \ldots \ldots \ldots \ldots$

2.8 Anisotropy in viscosity coefficients $\ldots \ldots \ldots \ldots \ldots \ldots$

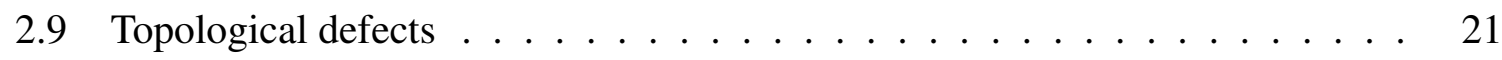

2.10 Optical micrograph of point defects $\ldots \ldots \ldots \ldots \ldots \ldots 22$

2.11 Nematic textures . . . . . . . . . . . . . . . . . . . . . 23

2.12 Topological defects at my finger tip . . . . . . . . . . . . . . . . 24

2.13 Viscosities in nematodynamics . . . . . . . . . . . . . . . . . . . 27

2.14 Transverse pressure gradient in Poiseuille flow of nematic LCs . . . . . 30

2.15 Effect of backflow on defect speed $\ldots \ldots \ldots \ldots \ldots$

2.16 Nematic colloids . . . . . . . . . . . . . . . . . . . . . . . . 34

2.17 Self-assembled structures in nematic colloids . . . . . . . . . . . . . 36

$3.1 \quad$ Material compatibility . . . . . . . . . . . . . . . . . . . 39

3.2 Long chain silane molecule, DMOAP $\ldots \ldots \ldots \ldots$

3.3 Fabrication of microfluidic devices $\ldots \ldots \ldots \ldots . \ldots . \ldots 42$

3.4 PDMS-glass microchannel and flow equipments . . . . . . . . . . . . 43

3.5 Polarization optical microscopy $\ldots \ldots \ldots \ldots \ldots \ldots$

$3.6 \quad$ Optical retardation using $\lambda$ plate $\ldots \ldots \ldots \ldots \ldots$

$3.7 \quad$ Fluorescence confocal polarization microscopy $\ldots \ldots \ldots$

3.8 Flow measurement using 2fFCS technique $\ldots \ldots \ldots \ldots \ldots \ldots$ 
4.1 Cartesian coordinates defining the micro-flow . . . . . . . . . . . 55

4.2 Wettability of 5CB on glass and PDMS surfaces . . . . . . . . . . . 56

$4.3 \quad$ LC anchoring on untreated glass surface . . . . . . . . . . . . . . . . . . 57

4.4 LC anchoring on untreated PDMS surface . . . . . . . . . . . . . . . 58

4.5 Degenerate planar anchoring in microchannels . . . . . . . . . . . . . . 60

4.6 Photoalignment technique to generate uniform planar surface anchoring . . . 61

4.7 Uniform planar anchoring in microchannels using photoalignment method . . 62

$4.8 \quad$ FCPM intensity distributions for functionalized microchannels . . . . . . . . 63

4.9 Uniform planar anchoring using pneumatic buffing . . . . . . . . . . . . . . . 64

4.10 Homeotropic anchoring in microchannels . . . . . . . . . . . . 65

4.11 Microchannels functionalized for hybrid orientation . . . . . . . . . . . 67

4.12 Fluorescence intensity distribution within a hybrid aligned microchannel . . . 68

5.1 Static director equilibrium in channels having uniform planar anchoring . . . 71

5.2 Nematic 5CB confined within a homeotropic microchannel . . . . . . . . 73

5.3 Influence of aspect ratio on nematic director equilibrium . . . . . . . . . . 74

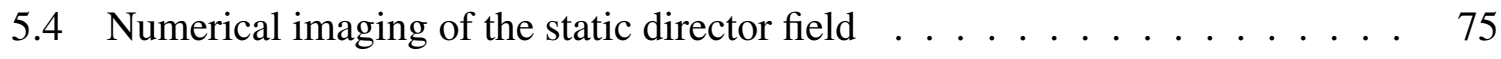

5.5 Topological defect line parallel to the channel wall $\ldots \ldots$. . . . . . . 76

5.6 Director configuration at channel corners . . . . . . . . . . . . . 77

$5.7 \quad$ Microfluidic confinement with cylindrical micro-pillar . . . . . . . . . 78

$5.8 \quad$ POM and FCPM imaging of the confined NLC around the micro-pillar . . . . 79

5.9 Director configuration around the micro-pillar . . . . . . . . . . . . 80

5.10 Defect loop around a micro-pillar . . . . . . . . . . . . . . . 80

5.11 Microfluidic confinement for hybrid alignment . . . . . . . . . 81

5.12 Equilibrium of the nematic director in hybrid alignment conditions . . . . . . 82

6.1 Pressure-driven nematic flow . . . . . . . . . . . . . . . . . 87

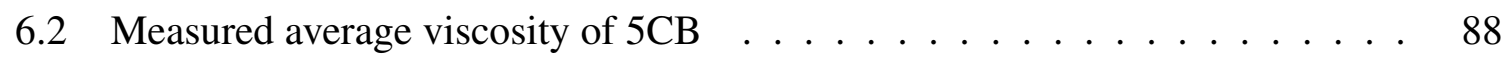

6.3 Composite microchannel . . . . . . . . . . . . . . . . . 9 90

6.4 Flow and nematic-to-isotropic phase transition . . . . . . . . . . . . . 91

6.5 Microchannel schematic showing degenerate planar anchoring . . . . . . . . 92

6.6 Stages of formation of a $\pi$-wall due to the nematic flow . . . . . . . . . . 93

6.7 Flow-induced textures in parameter space of velocity and channel depth . . . 94

6.8 Morphology of a $\pi$-wall created from hyperbolic -1 disclination line . . . . . 96

6.9 Morphology of a $\pi$-wall created from $+1 / 2$ disclination line . . . . . . . . . . 97

6.10 Manipulation of stable $\pi$-wall structures . . . . . . . . . . . . . . . 99 
6.11 Morphology of a surface-stabilized disclination . . . . . . . . . . . . 101

6.12 Flow alignment in microchannels with homeotropic surfaces $\ldots . . . . . .102$

6.13 No-flow nematic profile in a rectangular homeotropic channel . . . . . . 103

6.14 Three basic flow regimes in the homeotropic microchannel . . . . . . . . . 104

6.15 Experimental flow profiles of the weak, medium, and strong flow regimes . . 105

6.16 Numerical flow profiles of the weak, medium, and strong flow regimes . . . . 106

6.17 de Gennes length scales calculated from the experiments . . . . . . . . . 107

6.18 Steering of the micro-flow profile by a transverse temperature gradient . . . . 108

6.19 Nematic flow within a diverging homeotropic microchannel . . . . . . . . 110

6.20 Perturbation of the nematic director $\ldots \ldots \ldots \ldots \ldots$

6.21 Fluorescence intensity distribution due to director perturbation . . . . . . . 112

6.22 Evolution of birefringent domains with flow . . . . . . . . . . . . . 114

6.23 Director deformation with flow . . . . . . . . . . . . . . . . . . 115

6.24 Evolution of defect structures in nematic flow past an obstacle . . . . . . . 119

6.25 Extension of the singular loop $\ldots \ldots \ldots \ldots \ldots$

6.26 Transition to flow alignment $\ldots \ldots \ldots 121$

6.27 Evolution of wall defect in a shallow channel $\ldots \ldots \ldots$

6.28 Evolution of wall defect in a deeper channel . . . . . . . . . . . . . . . 124

6.29 Length of the defect wall . . . . . . . . . . . . . . . . . . . . . 125

6.30 Wall velocity . . . . . . . . . . . . . . . . . . . . 126

6.31 Defect sequence on flow reversal $\ldots \ldots \ldots \ldots \ldots$

6.32 Velocity distribution across the wall defect . . . . . . . . . . . . . . . 129

6.33 Collapse of disclination towards a channel corner . . . . . . . . . . 132

6.34 Laying down the 'soft rail' . . . . . . . . . . . . . . . . . . . . 133

6.35 Generation of disclination lines while filling the channel $\ldots \ldots \ldots$

6.36 Instability of the disclination line . . . . . . . . . . . . . . 135

6.37 Lateral positioning of the disclination line . . . . . . . . . . . 136

6.38 Navigating the disclination at a flow bifurcation $\ldots \ldots \ldots$

6.39 Role of secondary pressure gradient $\ldots \ldots \ldots \ldots$

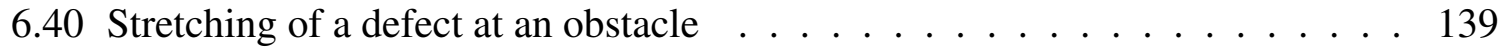

6.41 Effect of surface anchoring on topological defect population . . . . . . . 141

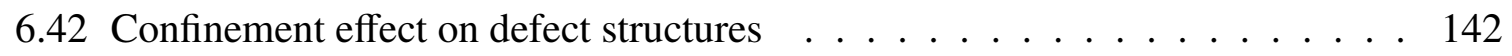

7.1 Micro-cargo transport in degenerate planar microchannels $\ldots . . . . . .144$

7.2 Trap-and-transport processes $\ldots \ldots \ldots \ldots$. . . . . . . . . . . 146 
7.3 Particle-disclination interaction measurement . . . . . . . . . . . . . . . . . 147

7.4 Director-field mapping by colloids in flow . . . . . . . . . . . . . . . . . . 148

7.5 Stereo-selective transport of nematic colloids . . . . . . . . . . . 150 


\section{List of Tables}

2.1 Anisotropic viscosity coefficients due to Miesowicz . . . . . . . . . . 20

3.1 Thermodynamic properties of $5 \mathrm{CB} \ldots \ldots \ldots \ldots \ldots$

3.2 Viscous and elastic of $5 \mathrm{CB} \ldots \ldots \ldots \ldots \ldots$

3.3 Leslie coefficients of $5 \mathrm{CB} \ldots \ldots \ldots \ldots \ldots$

3.4 Optical birefringence of $5 \mathrm{CB} \ldots \ldots \ldots \ldots$

$4.1 \quad$ Dynamic contact angle of 5CB on functionalized surfaces . . . . . . . . . 59

4.2 Thickness of polymer deposition on different substrates $\ldots \ldots \ldots$ 



\section{Chapter 1}

\section{Introduction}

\subsection{Liquid crystals: Complex anisotropic fluids}

Fluids: Simple and complex, are ubiquitous in our day-to-day life. While water, for all practical reasons, is a simple fluid exhibiting Newtonian behaviour, most of the food, personal care products and polymer solutions are complex non-Newtonian fluids. Complex fluids can be broadly considered as 'deformable solids' with physical attributes intermediate between solids and liquids. Typically, at short time scales the solid properties manifest more prominently over the liquid ones. However, at long times - from fractions of seconds to hours or days - the overall flow properties become visible. These different time scales define the characteristic non-linear mechanical response (deformation) of complex fluids to shear stresses and render them viscoelastic [1]. The non-Newtonian behaviour of fluids has been exploited in a number of engineering applications. Particularly, the use of tiny amounts polymers (few ppm) in water astonishingly reduce the turbulent drag [2], thereby significantly lowering the pumping power required for the flow. Furthermore, such non-Newtonian polymer solutions can lead to elasticity-mediated turbulent flows [3] even at low Reynolds number microfluidic flows [4]. Viscoelastic fluids like the polymer solutions exhibit these special properties due to breaking down of the internal fluid structures by flow, finally resulting in the change in the entropic elasticity [5].

Liquid crystals are mesophases of rod-like or disk-like molecules, intermediate between ordered solid phase (crystalline) and disordered liquid phase (isotropic). While classical viscoelastic liquids are isotropic, liquid crystals constitute a special class of complex fluid possessing anisotropic properties due to the spontaneously broken internal symmetry [6]. On one 
hand they possess hydrodynamic properties of complex fluids, on the other hand, they exhibit anisotropy in physical properties due to the inherent ordering existing in the mesophase. Hence, the anisotropy in elasticity, viscosity, and other physical properties of liquid crystals arises due to their orientational order, and is crucial for devising different kinds of applications, e. g. liquid crystal displays. Consequently, the flow of such anisotropic fluids is not only more complex due to the coupling between the liquid-crystal-ordering and the flow field, but also provides a physically rich system to investigate different competing effects characteristic to these systems.

\subsection{Microfluidics}

Microfluidics is a miniaturized and versatile platform to study the flow through micronsized confinements, typically capillaries, channels, or a network of such conduits. While the miniaturization owes to the micron or sub-micron dimension of the individual conduits, transporting fluids in volumes of microliter $(\mu \mathrm{L})$, nanoliter $(\mathrm{nL})$, or picoliter $(\mathrm{pL})$, the versatility of this platform is due to the wide range and highly diverse set of investigations that can be carried out in them. Since its emergence in the early 1990s [7], microfluidics has seen an ever-expanding reach in practically all fields of modern science and technology. Thereby, it has significantly contributed to the development of interdisciplinary research - converging physics, chemistry, biology, and technology - all on one platform.

Microfluidics derives its forte from the ability to control and manipulate flows precisely. Using a variety of techniques available [7], the conduits can be fabricated with high dimensional precision with or without morphological structures for flow manipulation: micropumps, micro-valves, and flow-guiding paths etc [8]. The microfluidic devices can be seamlessly integrated to an external field for enhanced functionality [9], or to existing flow characterization techniques [10]. The individual components can be subsequently put together to construct large-scale-integrated networks of varied levels of complexity, resulting in highly efficient lab-on-a-chip devices [11].

\subsection{Motivation}

Considering the umpteen possibilities microfluidic techniques can offer, my decision to investigate liquid crystals flowing through micron-sized confinements was rather straightfor- 
ward. The use of an anisotropic fluid as a continuous phase could offer possibilities beyond microfluidics based on isotropic fluids. However, quite astonishingly, a consequent review of the existing literature (till 2009, when I started my doctoral research) on liquid crystal flows revealed that this domain was practically unexplored. Nevertheless, the fundamentals of liquid crystal flows were already laid down by the numerical works of Ericksen [12, 13], Leslie [14, 15], and Parodi [16]. Almost parallely, a combination of numerical and experimental investigations were undertaken by the Orsay Group in Paris. While Dubois-Violette and Manneville [17-19] provided the numerical support during the 1970s, it was the experimental works of Pieranski and Guyon [20-23] that provided significant insights to the practical aspects of liquid crystal flows. A period of lull followed till the novel colloidal interactions in liquid crystals were discovered by the seminal work of Poulin et al. in the late 1990s [24]. Several numerical investigations were undertaken thereafter, especially on flow past inclusions [25] and the effects of flow on topological defects [26] around the inclusions [27--29].

In the more recent past, microfluidics was starting to be used as a tool to generate liquid crystal droplets [30-32], to study their wonderful properties [33-36], and to investigate confinement and motion of topological defects [37-40], and potential applications [41]. However, the possibility to use the available microfluidic techniques for studying the fundamental behaviour of liquid crystal flows within minute confinements was never explored. Especially, the effects of multiple surfaces in close proximity, and the influence of different surface properties on them, could be studied in a great detail by exploiting the precision microfluidics offered. Due to lack of adequate fabrication techniques previously, the fundamental experimental studies by Pieranski et al. were conducted within confinements with only one characteristic length, i. e. the channel depth, typically few hundred micrometers. A set of promising experiments investigating the effect of confinement and surface properties were initiated by Sambles and co-workers in Exeter, UK [42-44] using wide channels (width $\gg$ depth $\approx 30 \mu \mathrm{m}$ ).

Thus, in this doctoral thesis, I have tried to conduct the first systematic experiments in strict microfluidic confinements, and to explore the possibilities of novel applications based on liquid crystal microfluidics. Investigations were carried out for different surface functionalities within channels having a range of depths, from $5 \mu \mathrm{m}-100 \mu \mathrm{m}$, and aspect ratios. The promising outcomes provided subsequent impetus to investigate flows within channels of different geometries and flow past microscopic obstacles. The ability to control the generation and navigation of flow-induced topological defects led to the idea of controllably guiding colloids and droplets using defect lines as soft rails. However, the number of prospective possibilities which emerged during the course of this work outweighs that of the realized ones. 
Due to the distinct yet generic capabilities offered by the anisotropic liquid crystal microfluidics vis-à-vis isotropic microfluidics, I consider this work still at its infancy.

\subsection{Thesis outline}

The thesis is divided into nine chapters. Chapter 2 aims to provide an introduction to the physics of liquid crystals in general, and to that of the nematic phase in particular. The fundamental concepts of order parameter and the Landau-de Gennes theory are covered in the initial part of this chapter, followed by concepts of surface anchoring, anisotropy in liquid crystals, and topological defects. Thereafter, the theory of nematic liquid crystal flows is introduced. The chapter concludes with the theory of dispersions of nematic liquid crystals.

Chapter 3 gives a comprehensive description of the materials and the experimental methods employed in this work.

Chapters 4-7 constitute the main results of the thesis. In Chapter 4, we shall discuss a variety of physico-chemical techniques which has been developed to functionalize the walls of the microchannels for investigating the flow of nematic liquid crystals. Chapter 5 focuses on the confinement-induced equilibrium of the nematic liquid crystal within functionalized microchannels and about cylindrical micro-pillars. Additionally, we shall look into the effect of the channel aspect ratio on the equilibrium orientation of the nematic molecules. The outcome of a flow within the microfluidic confinements is presented in Chapter 6. The results are described within the parameter space of surface anchoring properties and channel dimensions. Finally, in Chapter 7, we shall look into nematic dispersions in microfluidic environment. A number of applications based on liquid crystal microfluidics is presented and proposed in this chapter.

In Chapter 8 , I shall discuss some of the ongoing investigations on microfluidics of nematic liquid crystals. Additionally, a perspective view on this field of research, especially addressing the open questions and the potential of liquid crystal microfluidics in applications, is presented, before concluding the thesis with some final remarks in Chapter 9 


\section{Chapter 2}

\section{Liquid crystal theory}

The basic theoretical approaches concerning liquid crystals and liquid crystal flows are presented in this chapter. The first half briefly reviews the concepts of order parameter and the Landau-de Gennes free energy. This will be followed by the concepts of surface anchoring, anisotropy, and the topological theory of defects. The second half focuses on the nematodynamic theory of liquid crystal flows, and the interaction between flow and topological defects. The chapter concludes with a short review on liquid crystal dispersions.

\subsection{Liquid crystal mesophases}

Matter exists in different states such as solid, liquid or gas. The distinction between the states is made by the degree and type of ordering the building blocks of matter - the molecules - exhibit with respect to their neighbours. While crystalline solids have highly ordered structures, gases do not show any positional or orientational order at all. The liquid state possesses only short-range, but no long-range ordering. Consequently, liquids have the highest possible symmetry, and crystalline solids a significantly lower symmetry [45]. In between solid and liquid states, there exists an intermediate mesophase, which exhibits long-range orientational order. Sometimes these mesophases can have an additional positional order. Liquid crystals (LCs) are such mesophases, comprising molecules with high shape-anisotropy. Due to the high asymmetry in shape, LC molecules are generally modeled as rigid rods or ellipsoids of revolution, as shown in Fig. 2.1 a. Broadly, liquid crystalline materials can be divided into two classes: thermotropic LCs and lyotropic LCs. While in thermotropic LCs the characteristic ordering depends only on temperature, the ordering in lyotropic LCs - typically formed 


\section{(a)}

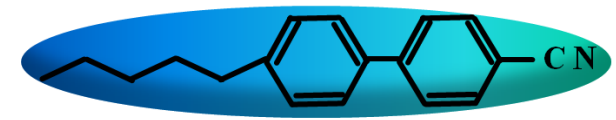

(b)

(X)

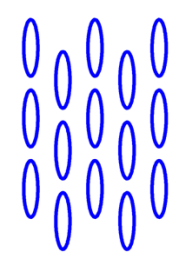

$T_{X S m}$

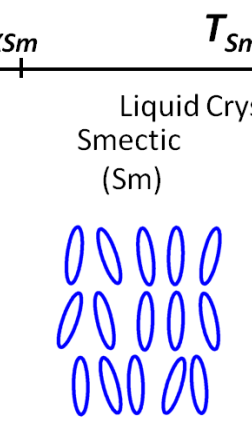

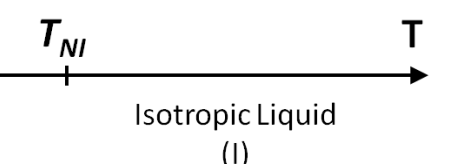

(I)

(N)

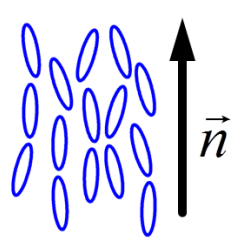

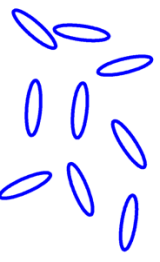

Figure 2.1: Shape anisotropy and liquid crystal phases. (a) Anisotropic shape of a typical liquid crystal molecule. The molecule shown here is Pentylcyanobiphenyl, commonly known as $5 \mathrm{CB}$, which exists in the nematic phase at room temperature. (b) Phase transition with temperature $(T)$ variation ( $T$ increasing from left to right). The liquid crystal phases exist within a particular temperature range $T_{X S m}<T_{L C}<T_{N I}$. Within this temperature range, different LC phases can exist, e. g. smectic $(S m)$ and nematic $(N)$. Vector $\vec{n}$ denotes the local director field.

by aqueous solution of amphiphilic molecules (surfactants) [46] - additionally depends also on the surfactant concentration. Thermotropic LCs are oily to touch, and are composed of organic molecules. Available either as single component compounds or as multi-component mixtures, they account for $90 \%$ of the world's display market in the form of liquid crystal displays (LCDs).

At high temperatures, the axes of the molecules randomly orient, resulting in the isotropic phase (Fig. 2.1 b). On cooling, the nematic phase nucleates first. Characterized by orientational order but no positional order, the nematic phase is the least ordered of the mesophases [47]. The molecules in the nematic phase on average align parallel to a particular direction defined by the unit vector $\vec{n}$ called the director [6]. This is indicated by the arrow in Fig. 2.1b. The nematic liquid crystalline phase can be categorized on the basis of the structure of the constituent molecules: calamitic nematic and discotic nematic. The calamitic nematic materials are formed by molecules which have rod-like structure, whereas, the discotic nematic phase comprises disc-shaped molecules that stack up one over the other. Commonly, the discotic nematic phase has a high tendency to form columnar phase. A variant of the nematic phase is the cholesteric phase or the chiral nematic phase, in which the director changes its direction 
in a helical fashion [48]. Very often a chiral nematic phase is obtained by doping the nematic mesophase with a chiral molecule (e. g. cholesterol nonanoate). On cooling the sample further, a second mesophase having positional ordering evolves in certain compounds. This is known as the smectic phase [49]. The segregation of the molecules into planes (Fig. 2.1p) leads to the additional ordering in smectic LCs. Depending upon the extent of ordering, smectic liquid crystals are further categorized as smectic $\mathrm{A}$, smectic $\mathrm{B}$, and smectic $\mathrm{C}$ phases. While smectic $\mathrm{A}$ and $\mathrm{C}$ phases retain their fluidity as an essential feature, the smectic B phase manifests as a lamellar phase with apparent similarities with traditional crystalline solids. On going down in temperatures, eventually the crystalline state is recovered. Such a temperature cycle is reversible, and by temperature stabilization, a specific mesosphase can be equilibrated. In this present work a room-temperature nematic mesophase has been used.

Historically, the first liquid crystalline compound was discovered by the Austrian botanist Friedrich Reinitzer in 1888 [50]. He had observed that cholesteryl benzoate on heating first showed a turbid liquid state, which on further heating produced a clear liquid. Surprised by this unusual melting behaviour, Reinitzer had consulted the German physicist Otto Lehmann, who carried out the optical characterizations of the turbid phase. Using polarization microscopy, Lehmann concluded that the turbid liquid could rotate the polarization state of the transmitted light and exhibited optical birefringence. Due to the apparent similarities with crystalline materials, Lehmann coined the new term crystalline liquid for this material [51]. Although similar observations followed for other fluids, it was only in 1922 that the French crystallographer Georges Friedel convincingly argued that liquid crystals represented a new state of matter, and the observations were not a mere coincidence [50]. Further investigations revealed that liquid crystals were more ubiquitous than previously thought. LC phases were identified in phospholipid cell membranes, a lipid material protecting the nerves, and even in some concentrated DNA and protein solutions, e. g. in the secretion of a spider that is used to generate silk. In modern world, LCs are omnipresent. Besides its popularity as display materials, they are present in high strength plastics, snail slime, detergents, textile fibers, components of crude oil, insect wings, eye shadow and even lipstick [52]. The scientific interest in LC materials was fuelled by the diverse application potentials that these materials offered, especially for tunable optical devices such as LC based displays [53, 54]. Simultaneously, the development of LC theory initiated, most notable among them being the Maier-Saupe microscopical theory [55] and the de Gennes phenomenological model [56], based on the Landau theory. Over the last years, liquid crystals have emerged as a promising candidate for functionalized smart materials for controlled self-assembly, high response electro-optic devices, biological 
and biotechnological applications and polymer sciences. In addition, LC materials provide a unique platform to investigate cosmological interactions and evolutionary dynamics within a usual laboratory set up.

\subsection{Order parameter}

The distinction between an isotropic liquid and the nematic mesophase arises due to the extent of orientational ordering of the molecules. The order parameter is the quantifying parameter of this orientational order. Although the centers of mass of the nematic molecules are not long-range correlated, the average orientation of the molecules shows a long-range order, denoted by the director, $\vec{n} \equiv-\vec{n}$. The equivalence of $\vec{n}$ and $-\vec{n}$ signifies that no change occurs on turning the molecules upside-down (no ferroelectricity) in an ordinary nematic [57]. The director field can be further generalized by accounting for the spatial $(\vec{r})$ and temporal $(t)$ variations: $\vec{n}(\vec{r}, t)$. While, in experiments, the spatial variations arise due to the variation of surface properties or presence of localized fields, temporal changes become conspicuous during the processes involving equilibration of the director, for instance, at short times after a thermal quench.

Due to the thermal fluctuations, the individual rod-shaped nematic molecules are generally skewed off the director, $\vec{n}$. If the orientation of the rod-shaped molecules is characterized by a vector $\vec{u}$, along the long axis of the molecule, the fluctuations can be formally accounted or quantified - by the scalar order parameter, $S$. $S$ is evaluated as the ensemble average of the second Legendre polynomials of the scalar product between molecular $\vec{u}$ and $\vec{n}$.

Let us consider a cartesian coordinate system, with the director $\vec{n}$ parallel to the $z$ axis. The orientation of individual molecules, characterized by the vector $\vec{u}$, can be consequently parametrized with

$$
u_{x}=\sin \theta \cos \phi, \quad u_{y}=\sin \theta \sin \phi, \text { and } u_{z}=\cos \theta
$$

Here $\theta$ and $\phi$ denote the angular deviations of the molecule along the polar and azimuthal directions respectively. The probability $\mathrm{d} \Psi$ to find a molecule oriented within a solid angle $\mathrm{d} \Omega$ is given by:

$$
\mathrm{d} \Psi=\frac{1}{4 \pi} f(\theta, \phi) \mathrm{d} \Omega
$$




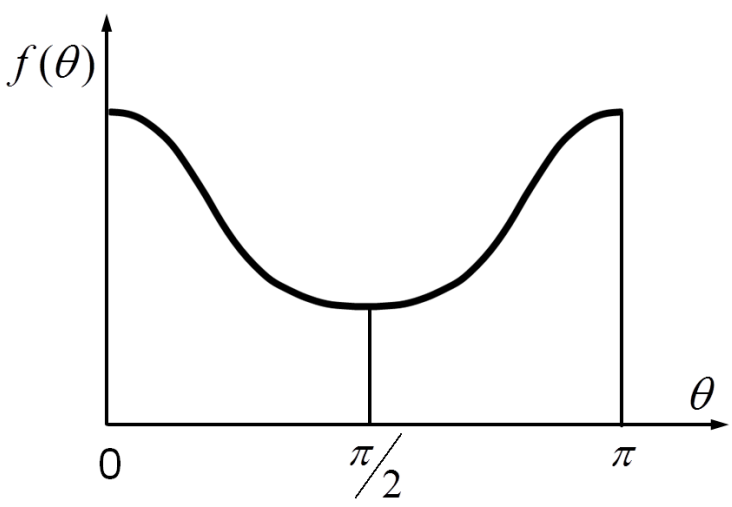

Figure 2.2: Generic shape of the distribution function $f(\theta, \phi)$ in an uniaxial nematic.

where $f(\theta, \phi)$ is the distribution function describing the general state of molecular orientation. The generic shape of $f(\theta, \phi)$ is shown in Fig. 2.2. Furthermore, for uniaxial nematics, which are axially symmetric about $\vec{n}, f(\theta, \phi)=f(\theta)$ holds good. In general, $f(\theta)$ can be expanded as a sum of the Legendre polynomials:

$$
f(\theta)=\sum_{n=0}^{\infty} f_{n} P_{n}(\cos \theta)
$$

Here, $P_{n}(x)$ is the $n^{\text {th }}$ Legendre polynomial and $f_{n}=\frac{2 n+1}{2} \int_{-1}^{1} f(\theta) P_{n}(\cos \theta) \mathrm{d}(\cos \theta)$.

Additionally, as a consequence of $\vec{u} \equiv-\vec{u}, f(\theta)=f(\pi-\theta)$. Hence, the non-zero contributions are due to the even terms only, with $f_{0}=1$. The scalar order parameter, $S$ is defined by the quadrupolar term:

$$
S=\frac{1}{5} f_{2}=\frac{1}{2} \int_{-1}^{1} f(\theta) P_{2}(\cos \theta) \mathrm{d}(\cos \theta)=\left\langle P_{2}(\cos \theta)\right\rangle
$$

The scalar order parameter consequently lies within the interval $-1 / 2 \leq S \leq 1$, where a value of $S=1$ indicates perfect ordering (all molecules along $\vec{n}$ ), and $S=0$ corresponds to the situation of maximum symmetry of isotropic state with no order. The negative value of the lower bound, $-1 / 2$, signifies an ordered state along a plane perpendicular to the director.

A single uniaxial tensorial order parameter, $\mathbb{Q}^{U}$, can be subsequently obtained by coupling the director and the scalar order parameter. Considering the orientational probability function in terms of $S$ up to the second order, we can obtain: 


$$
\begin{aligned}
f(\theta) & =1+5 S P_{2}(\cos \theta)=1+\frac{5}{2} S\left(3(\vec{n} \cdot \vec{u})^{2}-1\right)=1+\frac{5}{2} S\left(3 n_{i} n_{j}-\delta_{i j}\right) u_{i} u_{j} \\
& =1+\frac{5}{2} S\left(3(\vec{n} \otimes \vec{n})_{i j}-I_{i j}\right) u_{i} u_{j}=1+5 Q_{i j}^{U} u_{i} u_{j}
\end{aligned}
$$

Thus,

$$
\mathbb{Q}^{U}=\frac{1}{2} S(3 \vec{n} \otimes \vec{n}-\mathbb{I})
$$

Here, $\delta_{i, j}$ is the Kronecker delta and $I_{i, j}$ is the identity matrix, with $i, j$ denoting the summation over the repeated indices. $\mathbb{Q}^{U}$ is a symmetric traceless matrix, whose largest eigen value is the nematic order $S$, with the director $\vec{n}$ as the eigen vector. Two other directions $\vec{e}_{1}$ and $\vec{e}_{2}$ form an orthonormal triad with $\vec{n}$, and define the general order parameter tensor, $\mathbb{Q}$,

$$
\mathbb{Q}=\frac{1}{2} S(3 \vec{n} \otimes \vec{n}-\mathbb{I})+\frac{1}{2} B\left(\vec{e}_{1} \otimes \vec{e}_{1}-\vec{e}_{2} \otimes \vec{e}_{2}\right)
$$

where,

$$
B=\frac{3}{2}\left\langle\sin ^{2} \theta \cos 2 \theta\right\rangle
$$

signifies the sample biaxiality. Biaxility is generally observed in liquid crystal polymers and lyotropic liquid crystals. For a uniaxial nematic, like the one discussed in this work, $B=0$.

\subsection{Landau-de Gennes theory}

\subsubsection{Phase transition}

As we have briefly seen, the ordering in nematic mesophase can be altered by tuning the temperature. By varying the temperature significantly a phase transition can be effected, at which the mesophase microstructure and, consequently, the symmetry are considerably changed. Generally, this is accompanied by observable changes in physical properties like density, viscosity, and optical transmission. Thermodynamically, such a change implies change in the system entropy, given by:

$$
\mathrm{S}=-(\partial F / \partial T)_{V}
$$

where $F$ is the free energy defined at a given temperature $T$ and volume $V$. The order of 
the phase transition is determined by $\mathrm{S}$. A discontinuous variation of $\mathrm{S}$ with temperature is first order, whereas a continous variation of $S$ is a second order phase transition. The discontinuity in a first order transition results in exchange of latent heat $Q=T_{c} \Delta \mathrm{S}$ at the phase transition temperature $T_{c}$. The nematic-to-isotropic phase transition is a first order transition, characterized by the order parameter $S$ vanishing at the transition temperature, $T_{N I}$.

The transition can be described theoretically by using the Landau formalism. The free energy volume density reads as [6]:

$$
f=\frac{1}{2} a\left(T-T_{N I}^{*}\right) \operatorname{tr} \mathbb{Q}^{2}+\frac{1}{3} B \operatorname{tr} \mathbb{Q}^{3}+\frac{1}{4} C\left(\operatorname{tr} \mathbb{Q}^{2}\right)^{2}
$$

Here, $T_{N I}^{*}$ is the super-cooling temperature, and $a>0, B<0$, and $C>0$ are phenomenological material constants. The phase transition is driven by the temperature-dependent prefactor $\frac{1}{2} a\left(T-T_{N I}^{*}\right)$. The free energy functional can be rewritten in terms of the uniaxial order parameter tensor using the following relations:

$$
\begin{gathered}
\mathbb{Q}^{U}=\frac{1}{2} S(3 \vec{n} \otimes \vec{n}-\mathbb{I}) \\
\operatorname{tr}\left(\mathbb{Q}^{U}\right)^{2}=\frac{3}{2} S^{2} \text { and } \operatorname{tr}\left(\mathbb{Q}^{U}\right)^{3}=\frac{3}{4} S^{3} \\
f=\frac{3}{4} a\left(T-T_{N I}^{*}\right) S^{2}+\frac{1}{4} B S^{3}+\frac{9}{16} C S^{4}
\end{gathered}
$$

Equation 2.13 thus yields the dependence of the free energy within a given volume as a function of the scalar order parameter $S$. Clearly, the first term is responsible for the phase transition, the second term arises due to $S \neq-S$, and the third term provides the lower bound of $S$. Thus, the equilibrium of the system can be evaluated by minimizing the free energy for the entire volume $F=\int f \mathrm{~d} V$ at a given temperature. Consequently, the equilibrium order parameter is given by:

$$
S_{e q}=\frac{B}{2 C}\left(1+\sqrt{1-\frac{4 a C}{B^{2}}\left(T-T_{N I}^{*}\right)}\right), \forall T<T_{N I}
$$

Above the nematic-isotropic transition temperature, $S_{e q}=0$. Typical values of $a, B$, and $C$ are of the orders of $10^{5} \mathrm{~J} / \mathrm{m}^{3} \mathrm{~K},-10^{6} \mathrm{~J} / \mathrm{m}^{3}$, and $10^{6} \mathrm{~J} / \mathrm{m}^{3}$ respectively. Figure 2.3 a shows the variation of the Landau free energy density as a function of the scalar order parameter. $T_{N I}^{* *}$ refers to the highest temperature of the super-heated nematic phase. The general behaviour of 

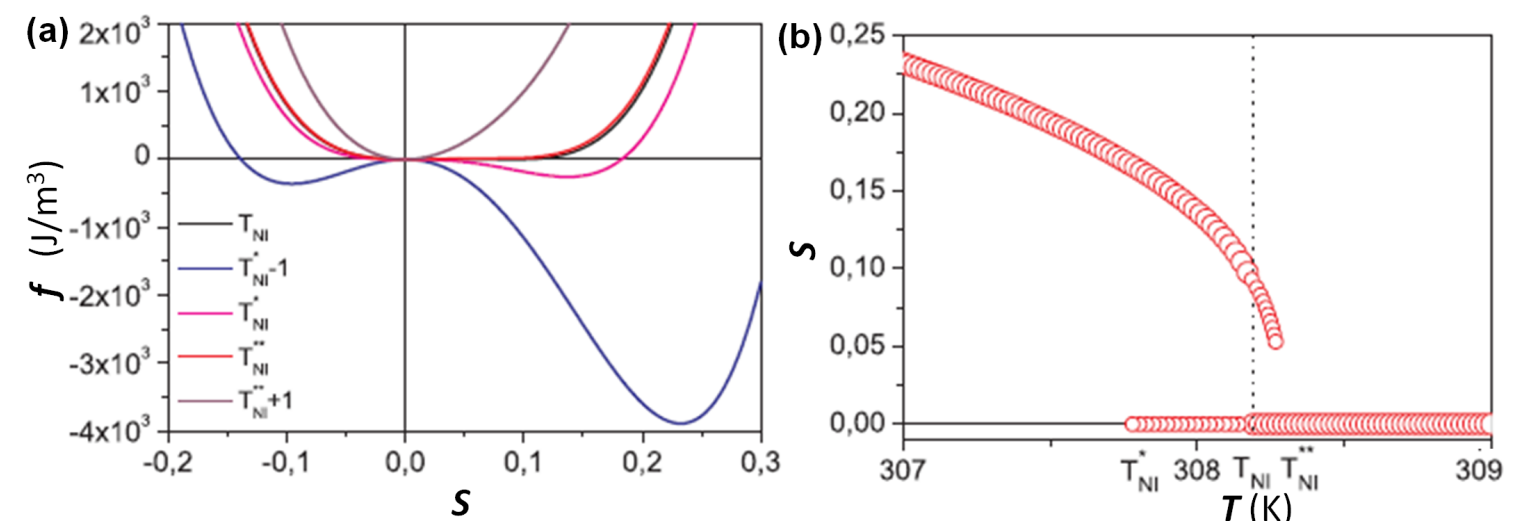

Figure 2.3: Free energy density and nematic order parameter variation. (a) Free energy density as a function of order parameter for different temperatures above and below $T_{N I}$. (b) Variation of the nematic order parameter as a function of temperature, shown here for nematic $5 \mathrm{CB}$. Adapted from [58].

the order parameter in a first order transition is shown in Fig. 2.3p. As shown, the order parameter vanishes at the nematic-isotropic transition temperature, $T_{N I}$. The Landau coefficients have been experimentally estimated by measuring the birefringence induced by a magnetic field (Cotton-Mouton effect) or equivalently by using an optical or electrical field [57].

\subsubsection{Nematoelasticity}

The Landau free energy discussed in Section 2.3.1 was derived assuming that the nematic is free of any external influence. However, this is seldom the case: Even the presence of the confining boundaries may significantly alter the overall free energy of the system. The surface-induced order is generally different from the equilibrium bulk value, and leads to elastic deformations in the director field. Typically, the nematic deformation falls into one or a combination of any of the basic modes: splay, twist, and bend. The deformations are schematically shown in Fig. 2.4.

In the limit of weak deformations, the free energy contributions corresponding to the elastic deformations can be formed out of the first order space derivatives $\partial Q_{i j} / \partial x_{k}$ of the order tensor and the elastic constants $L_{1}, L_{2}$, and $L_{3}$ [6]:

$$
f_{E}=\frac{1}{2} L_{1} \frac{\partial Q_{i j}}{\partial x_{k}} \frac{\partial Q_{i j}}{\partial x_{k}}+\frac{1}{2} L_{2} \frac{\partial Q_{i j}}{\partial x_{j}} \frac{\partial Q_{i k}}{\partial x_{k}}+\frac{1}{2} L_{3} Q_{i j} \frac{\partial Q_{k l}}{\partial x_{i}} \frac{\partial Q_{k l}}{\partial x_{j}}
$$




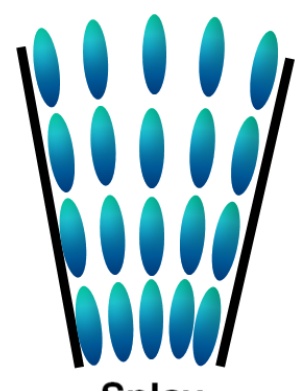

Splay

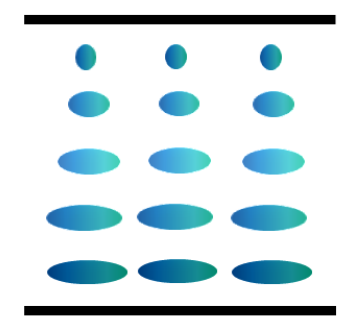

Twist

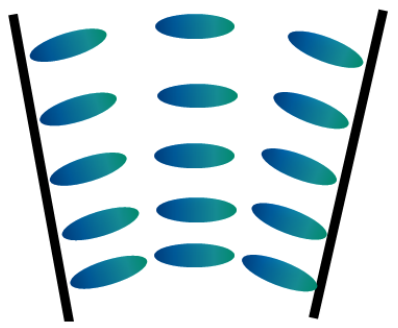

Bend

Figure 2.4: Elastic deformations in nematic liquid crystal (NLC): (a) splay, (b) twist, and (c) bend.

In terms of the director $\vec{n}$, the free energy expression yields the elastic energy density in the Frank-Oseen form [59]:

$$
f_{E}^{F O}=\frac{1}{2} K_{11}(\nabla \cdot \vec{n})^{2}+\frac{1}{2} K_{22}\left[\vec{n} \cdot(\nabla \times \vec{n})+q_{0}\right]^{2}+\frac{1}{2} K_{33}[\vec{n} \times(\nabla \times \vec{n})]^{2}
$$

where, $L_{1}=\left(K_{33}+2 K_{22}-K_{11}\right) / 9 S^{2}, L_{2}=4\left(K_{11}-K_{22} / 9 S^{2}\right.$, and $L_{3}=2\left(K_{33}-K_{11}\right) / 9 S^{2}$. Thus, the expressions of $K_{i i}$ inherently take into account the order parameter dependence of the nematic director deformation [60]. The basic modes of nematic deformation are directly addressed by the Frank elastic constants: $K_{11}$ signifies splay deformation with $(\nabla \cdot \vec{n})^{2} \neq 0$, $K_{22}$ corresponds to the twist deformation $[\vec{n} \cdot(\nabla \times \vec{n})]^{2} \neq 0$, and $K_{33}$ represents the bend deformation $[\vec{n} \times(\nabla \times \vec{n})]^{2} \neq 0$. The constant $q_{0}$ appearing in the twist deformation term is permitted only in systems lacking inversion symmetry, namely in the chiral phase. Further extensions can be incorporated in the Frank-Oseen free energy by the divergence of the energy terms [61, 62]:

$$
\begin{aligned}
& f_{13}=K_{13} \nabla \cdot[\vec{n}(\nabla \cdot \vec{n})] \\
& f_{24}=-K_{24} \nabla \cdot[\vec{n}(\nabla \cdot \vec{n})+\vec{n} \times(\nabla \times \vec{n})]
\end{aligned}
$$

$K_{13}$ and $K_{24}$ are mixed elastic constants and known as the splay-bend and saddle-splay constants respectively. Their effects are primarily limited to the surface of the nematic liquid crystals [46]. Furthermore, the Frank elastic constants are often simplified by assuming a one elastic constant approximation: $K_{11}=K_{22}=K_{33}=K \approx 6 \times 10^{-12} \mathrm{~N}$ for nematic 5CB. Under 
the one constant approximation, the Frank-Oseen free energy expression reduces to:

$$
f_{E}^{F O}=\frac{1}{2} K\left[(\nabla \cdot \vec{n})^{2}+(\nabla \times \vec{n})^{2}\right]
$$

\subsubsection{Landau-de Gennes free energy}

The Landau-de Gennes ( $\mathrm{LdG}$ ) formalism is a mean-field model for nematic liquid crystals that incorporates the free energy contributions from the nematic order and from the elasticity into a single functional:

$$
f_{L d G}=f_{E}+f
$$

The existence of the two contributing terms in the LdG formalism introduces a characteristic length of the variation of the nematic order. This length is known as the nematic correlation length $\xi_{N}$ and is evaluated by minimization of $f_{L d G}$ using the Euler-Lagrange formalism. Considering the one constant approximation of the elastic constants, the equilibrium condition for $S$ yields:

$$
\frac{3}{2} L \nabla^{2} S=\frac{\partial F_{L d G}}{\partial S}
$$

Assuming small spatial perturbations $\left(\Delta S\left(x_{i}\right) \propto \exp \left(\frac{x_{i}}{\xi_{N}}\right)\right)$ at equilibrium, the order parameter can be written as $S\left(x_{i}\right)=S_{e q}+\Delta S\left(x_{i}\right)$. Linearization of the Euler-Lagrange equation yields the nematic correlation length:

$$
\xi_{N}=\sqrt{\frac{3}{2} \frac{L}{\left.\frac{\partial^{2} f_{L d G}}{\partial S^{2}}\right|_{S_{e q}}}}=\sqrt{\frac{L}{a\left(T-T_{N I}^{*}\right)+B S_{e q}+\frac{9}{2} C S_{e q}}}
$$

The correlation length thus increases on approaching the transition temperature $T_{N I}$. Typically, $\xi_{N}$ is of the order of few nanometers. The Landau-de Gennes formalism is one of the most general models for characterizing LC phenomena. It is relevant for different length scales, boundary conditions, and can also be extended to cases in which external fields are involved. However, at nanometer scales, the model faces certain limitations owing to the mean-field approximations. 


\subsection{Surface anchoring}

In absence of any external fields or interacting surfaces, the equilibrium nematic director field is uniform, decided by the internal ordering of the mesophase. However, the presence of an interface: solid, liquid, or gas, can affect the inherent ordering, leading to a modified equilibrium state. Typically, the molecules in the vicinity of a surface attain an easy axis, along which they are locally biased to orient. Formally, this is represented by a surface-induced order parameter tensor, $\mathbb{Q}_{0}=\frac{1}{2} S_{0}\left(3 \vec{n}_{0} \otimes \vec{n}_{0}-\mathbb{I}\right)$, where $\vec{n}_{0}$ and $S_{0}$ denote the direction of easy axis and surface-induced order respectively. This additional order parameter signifies the state of molecular orientation on a given surface, the latter commonly known as surface anchoring [63]. Figure 2.5] shows different possible states of surface anchoring of nematic LCs. The first observation of surface anchoring was reported by Mauguin for a mica substrate [64]. The LC molecules in this case oriented uniformly parallel to the substrate plane. The other commonly encountered anchoring is homeotropic anchoring, where the molecules anchor perpendicular to the substrate plane (Fig. 2.5b). Different modifications of the basic anchoring states do occur in nature (Fig. 2.5; and d).

(a)

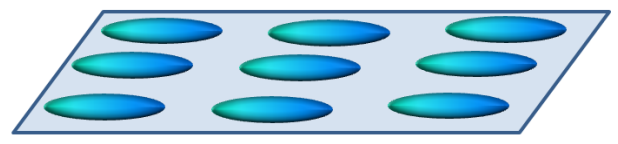

(c)

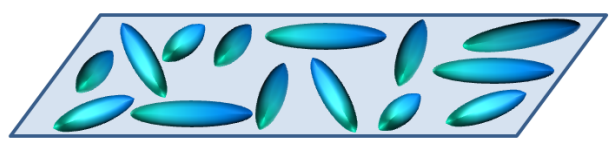

(b)

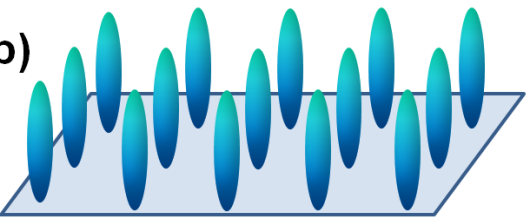

(d)

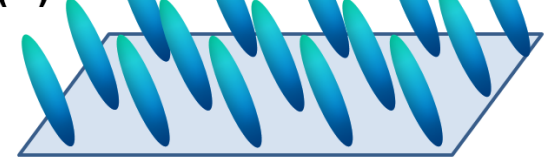

Figure 2.5: Anchoring of nematic liquid crystal on a surface: (a) uniform planar anchoring and (b) homeotropic anchoring. Different modified anchoring states can occur: (c) degenerate planar anchoring and (d) tilted anchoring.

In general, the director $\vec{n}$ alignment in absence of any elastic torque along the easy axis is determined by the interaction between the LC and the aligning surface. Orientation of the molecules on the surface is characterized using two angular components [65]: azimuthal anchoring $\varphi$ and zenithal anchoring $\theta$ as shown in Fig. 2.6. The angle $\varphi$ between the director projection and a reference direction on the substrate plane gives the measure of the azimuthal orientation of $\vec{n}$. The zenithal angle, frequently referred as pretilt angle, $\theta$ between $\vec{n}$ and the 


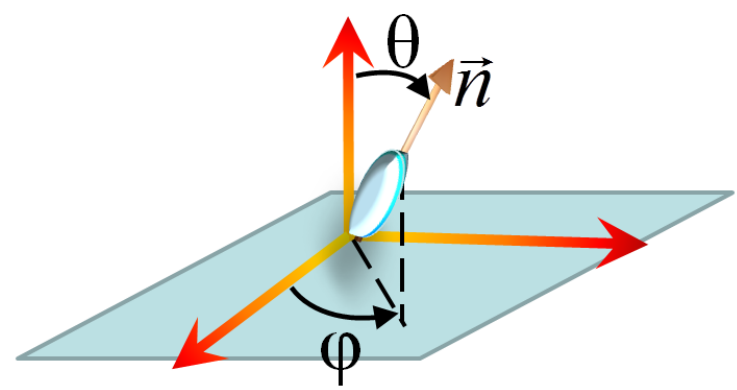

Figure 2.6: Schematic representation defining the two characteristic anchoring angles: polar anchoring angle $(\theta)$ and the azimuthal anchoring angle $(\varphi)$.

substrate normal gives the tilt of $\vec{n}$. Thus, orientation of the director represented in Fig. 2.5 $\mathrm{d}$ can be addressed in terms of the polar and the azimuthal angles: $\theta=\pi / 2, \varphi=$ constant for uniform planar, $\theta=0$ for homeotropic, $\theta=\pi / 2, \varphi=$ arbitrary for degenerate planar, and $0<\theta<\pi / 2, \varphi=$ constant for tilted anchoring. As a consequence of the long-range ordering, the orientation of $\vec{n}$ at the surface extends into the bulk material, minimizing the elastic energy of the system. A finite amount of energy is necessary to deviate the director $\vec{n}$ from the easy axis. This characterizes the anchoring strength quantitatively and is usually called the anchoring energy. For a nematic LC in confinements comprising surfaces with different anchoring, energy minimization leads to a continuous tilt of $\vec{n}(\vec{r})$, either globally or locally. This is known as hybrid alignment.

In practical situations, the actual surface ordering $\mathbb{Q}_{s}$ is typically different from $\mathbb{Q}_{0}$. The difference is accounted by the contribution of a surface term to the free energy. For uniaxial nematics, the surface contribution to the free energy thus reads:

$$
f_{s}=\frac{9}{8} W_{e}\left[\frac{2}{3} S_{s}^{2}+\frac{2}{3} S_{0}^{2}-2 S_{s} S_{0}\left(\left(\vec{n}_{s} \cdot \vec{n}_{0}\right)^{2}-\frac{1}{3}\right)\right]
$$

Here $W_{e}$ represents the strength of anchoring in units of energy. Typically, the anchoring energy varies from $10^{-3} \mathrm{~J} / \mathrm{m}^{2}$ (strong anchoring) to $10^{-7} \mathrm{~J} / \mathrm{m}^{2}$ (weak anchoring) [66]. The expression can be simplified further with $S_{0}=S_{s}$, yielding the Rapini-Papoular form [67]:

$$
f_{s}^{R P}=-\frac{1}{2} W_{e}^{R P} \cos ^{2} \alpha
$$

Clearly, from equation 2.24, one observes that the free energy density now depends only on $\cos \alpha=\vec{n}_{0} \cdot \vec{n}_{s}$, i. e. on the deviation of the nematic director with respect to the easy axis. 
Consequently, the anchoring energy can be evaluated from it.

In addition to $W_{e}$, the strength of anchoring on a substrate can be also described by a characteristic length scale, called the surface extrapolation length, $\xi_{S}$, due to Kléman and de Gennes [6, 68]. Defined as:

$$
\xi_{S}=K / W_{e}^{R P}
$$

the extrapolation length gives an estimation of the nematic elasticity in comparison to the surface anchoring. In the limit of strong anchoring, the extrapolation length is of the order of few nanometers. However, for surfaces possessing weak anchoring, this length may go up to few micrometers [69].

\subsection{Anisotropy in liquid crystals}

Liquid crystals provide a rather ready access to a class of complex fluids with anisotropic properties. From single-component organic LC compounds to multi-component LC mixtures, the mesophases exhibit anisotropy in a variety of physical properties. In this section, we shall discuss the general aspects of LC anisotropy and focus on two of them: optical anisotropy and anisotropy in viscosity, which we shall frequently come across in the subsequent chapters.

The anisotropy in LC phases stems from the anisotropic shape of the constituting molecules and the resulting ordering characteristic of the mesophases. In general, the magnitude of a physical property measured along the average molecular orientation, i. e. along the director vector $\vec{n}$, is different from that measured orthogonal to the director. For example, measurement of the Stokes drag on a particle in an NLC mixture conclusively shows the existence of anisotropic diffusion constants [70]. Similarly, anisotropic behaviour is observed in other properties as well: optical, magnetic, electrical and thermal conductivity, flow properties etc.

\subsubsection{Optical anisotropy}

The optical anisotropy in nematic mesophases is made manifest by the distinct values of the refractive indices along the optical axis (director): $n_{\|}$, and perpendicular to it: $n_{\perp}$. Most of the nematic and smectic phases, are optically positive: $n_{\|}>n_{\perp}$. Optical anisotropy is generalized by the indicatrix, an ellipsoid of revolution whose major and minor axes correspond to $n_{\|}$ and $n_{\perp}$ respectively. For an optically negative specie, $n_{\|}<n_{\perp}$, as shown in Fig. 2.7A. Further- 
(a)

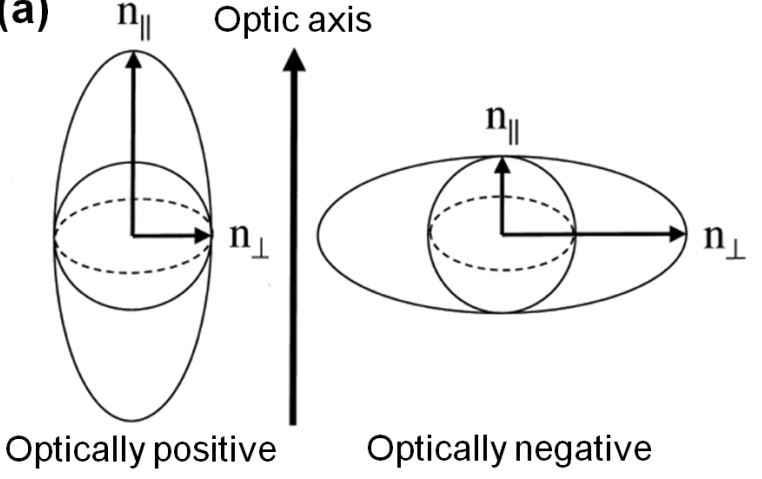

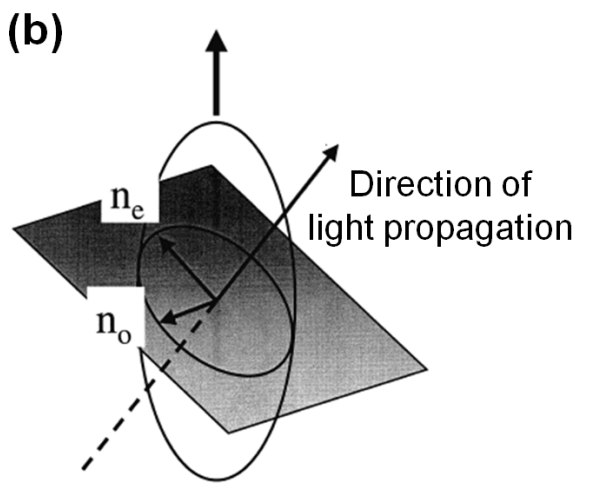

Figure 2.7: Optical anisotropy. (a) Schematic representing optically positive (left) and optically negative (right) samples. (b) Transmission of light through an optically anisotropic medium yields an ordinary $n_{o}$ and an extraordinary $n_{e}$ component. Adapted from [72].

more, uniaxial nematics are characterized by one principal optical axis, making them optically uniaxial [71]. Similarly, biaxial species have two principal axes, i. e. they are optically biaxial.

When a light beam is transmitted at some angle $\phi$ relative to the optic axis, it is split into two components: the ordinary ray having index of refraction, $n_{o}$, and the extraordinary ray having index of refraction, $n_{e}$. This is shown in Fig. 2.7b. The refractive indices corresponding to the ordinary and extraordinary components are related to $n_{\|}$and $n_{\perp}$ through the relative angle $\phi:$

$$
\begin{aligned}
& n_{e}=\frac{n_{\|} n_{\perp}}{\sqrt{n_{\|}^{2} \cos ^{2} \phi+n_{\perp}^{2} \sin ^{2} \phi}} \\
& n_{o}=n_{\perp}
\end{aligned}
$$

Consequently, the ordinary and extraordinary rays propagate through the sample at different speeds, resulting in a phase difference within a sample of optical distance $d$ :

$$
\delta=\frac{2 \pi}{\lambda}\left(n_{e}-n_{o}\right) d
$$

where $\lambda$ is the vacuum wavelength of the propagating beam. The parameter of particular interest here is the difference between the ordinary and extraordinary refractive indices, termed as the optical birefringence:

$$
\Delta n=n_{e}-n_{o}
$$


When a linearly polarized light is passed, it is converted into elliptically polarized light, with a component that can pass through a crossed polarizer, also referred as the analyzer [72]. The intensity of the light finally coming out beyond the analyzer is given by:

$$
I=I_{0} \sin ^{2} 2 \varphi \sin ^{2} \frac{\delta}{2}
$$

where, $I_{0}$ is the light intensity after the first polarizer, and $\varphi$ is the angle between the analyzer and the optic axis projection on the sample plane. While the first term in equation 2.30 quantifies the intensity of the light transmitted through the crossed polarizers on rotating the sample, the second term is responsible for the birefringent colours in thin nematic films. We shall revisit this while characterizing anchoring within microchannels using polarization microscopy in Chapter 4 .

\subsubsection{Viscosity}

Nematic liquids in general are shear thinning in nature [1]. When confined as thick samples (few hundred micrometers), an average bulk viscosity characterizes the rheological behaviour of the mesophase. However, as the confinement dimensions are progressively reduced, the surface-induced ordering increasingly contributes to the equilibrium director field. Perturbation of the equilibrium state by flow is then dependent not only on the direction of the flow field relative to the director field, but also on the relative configuration of the flow gradient and the director field. Hence, a set of viscosity coefficients are obtained depending upon the mutual orientation of the flow and director fields:

(i) $\vec{n}$ parallel to the flow direction: $\eta_{1}$,

(ii) $\vec{n}$ parallel to the gradient of flow: $\eta_{2}$, and

(iii) $\vec{n}$ perpendicular to the flow direction, and to the gradient of flow: $\eta_{3}$

Experimentally, this was first demonstrated by Miesowicz [73]. To measure the respective viscosity coefficients, the director field was stabilized using a strong magnetic field. Table 2.1 summarizes the results of the experiments conducted by him.

Subsequently other experiments have confirmed the observations of Miesowicz and added the effects of temperature on the viscosity anisotropy. Figure 2.8 shows the variation of the three viscosity coefficients with temperature for $p^{\prime}$-methoxybenzylidene- $p$ - $n$-butylaniline (MBBA) [74]. It can be seen that the anisotropy in viscosity is least just below the nematicto-isotropic transition temperature. As the temperature is decreased, the viscosity anisotropy 


\begin{tabular}{|c|c|c|c|}
\hline Substance and temperature & $\eta_{1}(\vec{v} \| \vec{n})$ & $\eta_{2}(\nabla \mathrm{v} \| \vec{n})$ & $\eta_{3}(\vec{v}, \nabla \mathrm{v} \perp \vec{n})$ \\
\hline \hline$p$-Azoxyanisol $\left(122^{\circ} \mathrm{C}\right)$ & $0.024 \pm 0.0005$ & $0.092 \pm 0.004$ & $0.034 \pm 0.003$ \\
\hline$p$-Azoxyphenetol $\left(144.4^{\circ} \mathrm{C}\right)$ & $0.013 \pm 0.0005$ & $0.083 \pm 0.004$ & $0.025 \pm 0.003$ \\
\hline
\end{tabular}

Table 2.1: Anisotropic viscosity coefficients measured by Miesowicz for two different LC materials. The director field was stabilized by a strong external magnetic field [73].

increases. Incidentally, the experiments of Gähwiller provide a direct method to evaluate the five independent coefficients which appear in the dissipative part of the stress tensor, formulated by Ericksen [12] and Leslie [14]. These five coefficients, $\alpha_{1}$ till $\alpha_{5}$, have the dimension of viscosity, and are known as the Leslie coefficients. In addition to these, one can define an effective coefficient of viscosity when the molecules undergo a rotational motion [75]. In Section 2.7 we shall see how the Miesowicz coefficients and the rotational viscosity, $\gamma$ can be derived from a combination of the Leslie coefficients.

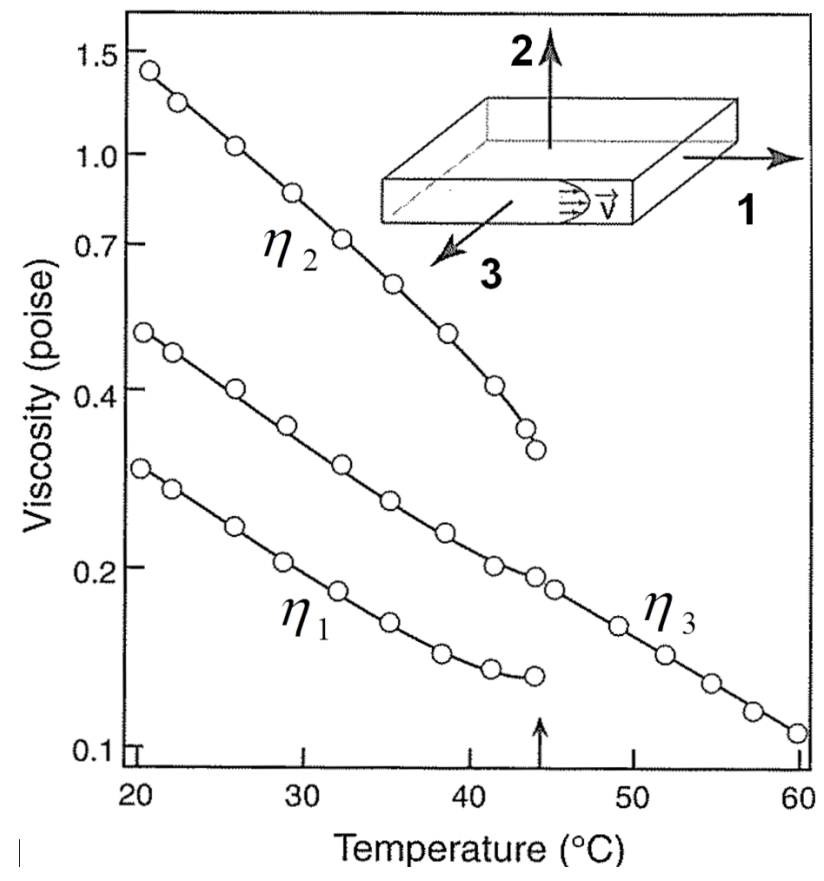

Figure 2.8: Anisotropy in viscosity coefficients. The plot shows three different viscosity coefficients measured for MBBA. Inset represents the velocity field relative to the director orientation along the three directions: 1, 2, and 3. Adapted from [76]. 


\subsection{Topological defects}

Topological defects [26, 77] abound systems with broken symmetries: in ordered media like magnetic materials, crystalline materials - both solids and liquids, in superfluid helium, and in quantum Hall fluids [45, 78, 84]. Owing to the similarities in nature of the scaling laws characterizing such systems, liquid crystals provide an easily accessible platform for investigations. Even certain cosmological models can be verified in the laboratory by simply studying the defect dynamics of liquid crystals [85, 86]. Incidentally, the current knowledge we have about defects (dislocation theory) in crystalline solids owes significantly to the early investigations which were carried out on liquid crystalline media by O. Lehmann, G. Friedel, and F. Grandjean [87-92]. Furthermore, numerous biological structures exhibit liquid crystalline ordering, motivating investigations about the origin of life based on optical microscopy of LCs [93].

(a)

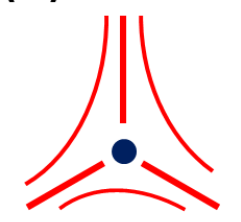

$-1 / 2$

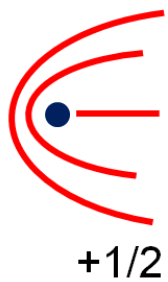

(b)

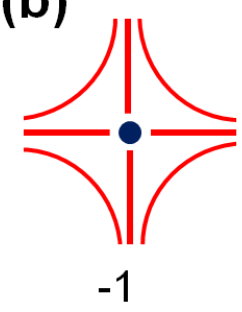

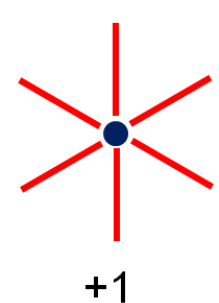

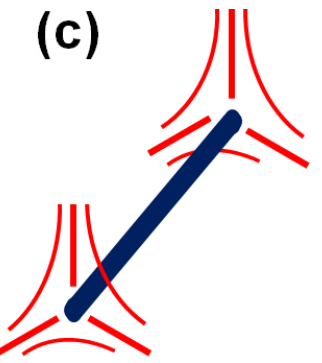

Figure 2.9: Topological defects. (a) Negative (left) and positive (right) point defects of topological rank 1/2 (semi-integer defects). (b) LC defects of topological rank 1. (c) Schematic representation of a disclination line with $-1 / 2$ defect core. Red lines indicate the director field in the vicinity of the defect core (blue).

Put simply, defects in liquid crystals can be defined as certain localized spots within the sample where the order parameter (and hence the director orientation) is ill-defined. Naturally, a disordered spot is a discontinuity or singularity within the otherwise ordered phase. The presence of the defects not only alters the physical properties in their vicinity, but also increases the overall free energy of the system. Consequently, an ideal ordered medium is free of defects. However, the influence of the surfaces, external fields, or a lowering of the symmetry (isotropic to nematic transition) can spontaneously and/or controllably create and stabilize topological defects. 

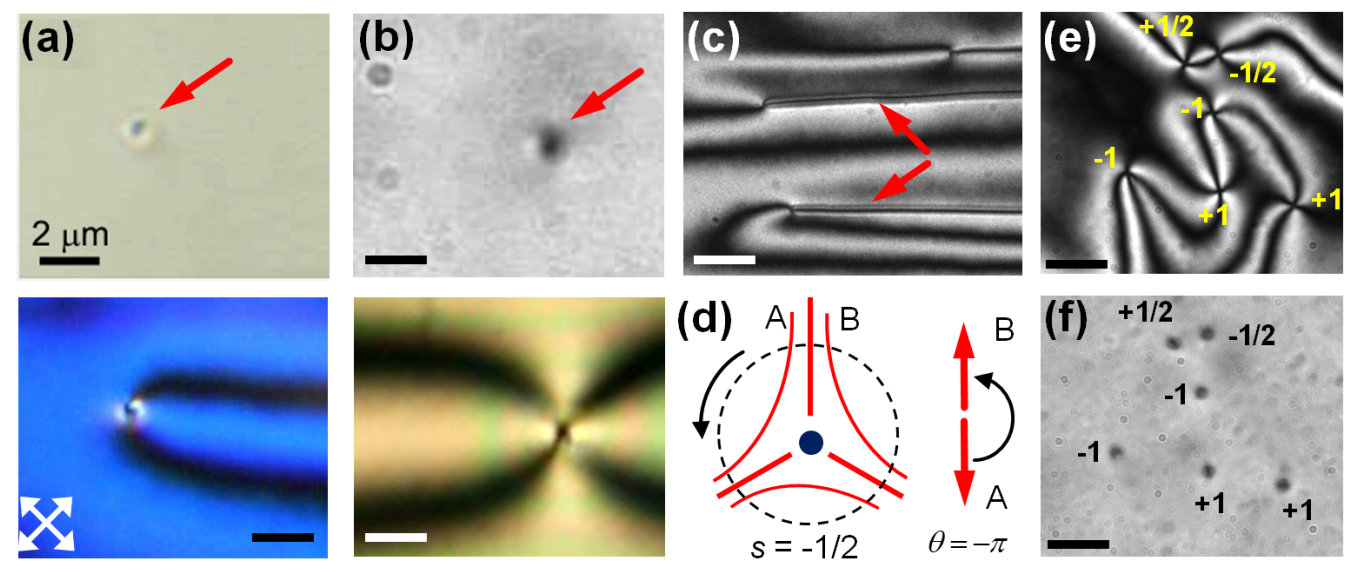

Figure 2.10: Optical micrograph of point defects. (a) Micrograph showing a semi-integer point defect viewed in white light (top) and between crossed polarizers (below). (b) Corresponding micrographs for an integer point defect. Note the scattering of light from the defect core in the micrographs (top panel). The polarizers are indicated by the crossed arrows. (c) Disclination lines originating at semi-integer point defects. (d) Burgers circuit constructed around a wedge defect. By traversing from A to B the director rotates by angle $\pi$. Additionally, the sense of the director at A and B are mutually opposite. (e) Polarization optical micrographs of a typical nematic texture comprising integer and semi-integer defect pairs. Corresponding white light micrograph is shown in (f).

The singular spots which constitute LC defects can be of various dimensions. If the singularity is at a point, it is called a point defect, which is a 0-dimensional structure (Fig. 2.9 a and b). Line defects are 1-dimensional structures, as shown schematically in Fig. 2.9c. They are widely found in LC systems: during the isotropic to nematic phase transition, or can be readily created by perturbing the liquid crystal sample, e. g. by simply stirring the LC with a spatula. Commonly referred to as disclinations (due to Frank [94]) or rotation dislocations [26], they are essentially distinct from crystalline dislocations. While in the latter the translation symmetry is broken along a line, it is the rotation symmetry that is broken in line defects in liquid crystals [46]. Defects can be generalized further as 2-dimensional structures in defect walls. When observed under optical microscopes, the defects appear as dark points or lines (as the case may be) due to the scattering of light at the defect core $(\approx 10 \mathrm{~nm}$ diameter). Owing to the reduced order within the defect core, the thermal fluctuation of the molecules is significantly higher than that of the ordered matrix. Consequently, the anisotropy of the ordered phase vanishes at the core, rendering the defects optically distinct. Figure 2.10 shows defect 


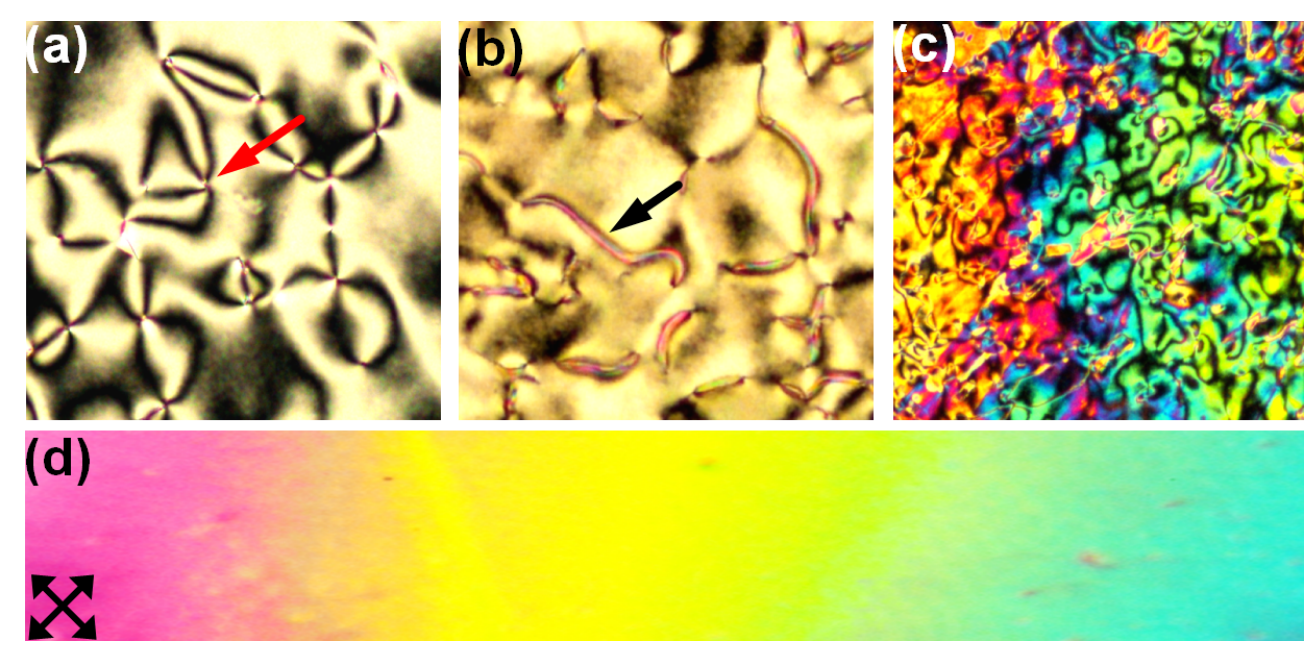

Figure 2.11: Nematic textures observed between crossed polarizers. (a) Schlieren texture consisting of integer and semi-integer point defects. (b) Nema or thread-like texture comprising disclination lines connecting the surfaces. (c) Schlieren texture observed in a thin film of nematic LC. The birefringent colours are characteristic of thin films. (d) Marble texture showing different birefringent domains. The domains were controlled by creating a gradient of film thickness.

structures commonly observed in nematic liquid crystals. Such singularities are often modeled as isotropic cores [48]. In the absence of uniform alignment, either surface induced or otherwise, many defects agglomerate within the nematic sample, giving rise to a variety of nematic textures. Figure 2.11 shows polarization micrographs of commonly observed textures. The observations were made for $5 \mathrm{CB}$ at room temperature without any external field.

Disclinations in nematic liquid crystals are broadly divided into two classes: wedge disclinations and twist disclinations [94, 95]. While the line singularity lies perpendicular to the plane of the molecules in the disclinations of the wedge type, the line lies in the plane of the molecules for the twist type disclinations. We shall however focus more on the wedge type disclinations because of two reasons: (a) defects in nematic liquid crystals are primarily wedge type, and (b) wedge type disclinations are much simpler to comprehend than their twist counterparts.

The concepts of rank and the charge of the topological defects can be explained by constructing a hodograph, an imaginary closed path, around the defect (positive sign in counterclockwise direction). It is also commonly referred to as the Burgers circuit [45], shown by the 
(a)

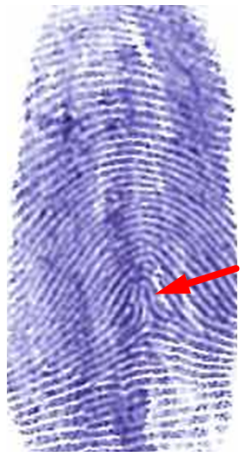

Semi-integer pair (b)

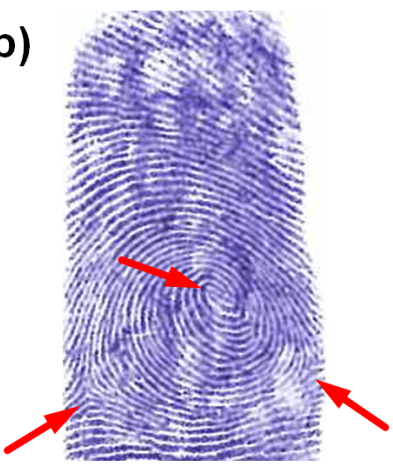

Integer and semi-integer

Figure 2.12: Topological defects at my finger tip. (a) Fingerprints on my index finger resemble a semi-integer defect pair $( \pm 1 / 2)$. (b) Fingerprints on my thumb comprise an integer $(+1)$ defect in the middle (center of the spiral) with two semi-integer defects on the periphery $(2 \times-1 / 2)$. Interestingly, the total topological charge is conserved.

dotted lines in Fig. 2.10d. Starting from a point on this path, over one complete angular trip of $2 \pi$, the director vector, $\vec{n}$ rotates by an angle $\theta$, given by:

$$
\theta=2 \pi s
$$

where $s$ is the topological rank of the defect, also frequently referred to as the winding number. Due to the director equivalence $(\vec{n} \equiv-\vec{n})$ in nematics, $s$ takes integer or semi-integer values. Depending upon the sense of rotation, $\theta$ can be positive or negative, thus determining the topological charge of the defects. Topological analysis of the defect shown in Fig. $2.10 \mathrm{~d}$ yields $s=1 / 2$ carrying a negative charge. Although topological defects can be energetically stable, usually they do not correspond to the lowest free energy state [48]. Under the one elastic constant approximation, the energy of an isolated dislination line per unit length is given by:

$$
E=\int_{r_{c}}^{R} f_{E}(2 \pi r) \mathrm{d} r=\pi K s^{2} \ln \left(\frac{R}{r_{c}}\right)
$$

Here, $R$ is the sample size, and $r_{c}$ is known as the core radius, which is usually of molecular dimensions. The quadrupolar order parameter within the core reduces to zero [96, 97]. Additionally, the average energy of the disclination varies as square of the topological rank, $s$. Hence, defects of higher ranks (integer defects) generally transform [98] into low energy 
semi-integer defect states or transform into a continuous director field through out-of-plane transformations [99]. In presence of multiple topological defects, the average separation between the lines as well as the topological charge are considered for estimating the energy. This will be discussed in Chapter 5. It is worthwhile to mention here that topological defects always occur in pairs with opposite signs. In essence this implies that the net topological charge of a system remains conserved. Interestingly, this is readily observed in our finger prints. As shown in Fig. 2.12, the finger prints share a close resemblance with the topological structures discussed so far. Indeed, the corresponding topological charges are conserved. Hence, in case of confined LC systems, the overall director field need to statisfy an additional condition of topological charge conservation. In Chapters 5 and 6 we shall see how topological constraints become apparent in determining the overall director configuration in absence or presence of flow fields. Furthermore, topological defects with opposite signs attract each other which might lead to annihilation of the defect pair, leaving behind a defect-free state [100]. In the following section, we shall see how flow, director, and topological defects interact with each other, due to the flow-director coupling, and thereby affect the overall dynamics of the system.

\subsection{Flow of nematic liquid crystals: Nematodynamics}

External perturbations are commonly employed to drive ordered fluids like nematic liquid crystals out of their thermodynamic equilibrium. Effected by electric, magnetic [101-104], optical [105-107], or flow fields, out-of-equilibrium states are ubiquitous in the realm of liquid crystal research and applications. While the dynamics are distinct (and more complicated) from those of an isotropic fluid, they are also rich with phenomena which have no analogy in isotropic fluids. Owing to the backflow mechanism - the coupling between the director field $\vec{n}$ and the velocity field $\vec{v}$ - NLCs provide capabilities beyond isotropic fluidics. Unlike isotropic fluids, perturbation of nematics alter the director alignment, and conversely, the director reorientation induces nematic flow.

A macroscopic theoretical approach of the flow-director coupling has been formalized by the works of Ericksen [12, 108], Leslie [14, 15], and Parodi [16]. The theoretical frame work is based on classical mechanics. However, a microscopic approach, was undertaken by the Harvard group using correlation functions. Later, this was extended to other mesomorphic phases using a macroscopic formalism [109-111]. The equivalence of these two different approaches was established by de Gennes [6]. In this section we shall have a concise look into 
the constitutive equations of nematodynamics due to Ericksen and Leslie. Further details and derivations of the equations can be found in [75, 76, 112].

\subsubsection{Ericksen-Leslie theory of nematodynamics}

Let us consider the nematic director orientation, $\vec{n}$, in cartesian coordinates. In terms of $\theta$ and $\phi$, which define the polar and azimuthal angles, the director vector parametrization yields:

$$
\vec{n}=(\sin \theta \cos \phi, \sin \theta \sin \phi, \quad \cos \theta)
$$

The velocity field $\vec{v}$ in terms of its components $(u, v$, and $w)$ and the pressure $p$ is written as:

$$
\begin{aligned}
\vec{v} & =(u(x, y, z), v(x, y, z), w(x, y, z)) \\
p & =p(x, y, z)
\end{aligned}
$$

Thus we can write the conservation equations involving the mass, linear momentum, and angular momentum terms:

$$
\begin{gathered}
v_{i, i}=0 \\
\rho F_{i}-\tilde{p}_{, i}+\Gamma_{k}^{v i s} \frac{\partial n_{k}}{\partial \theta} \theta_{, i}+\tilde{\sigma}_{i k, k}=0 \\
\left(\frac{\partial W}{\partial \theta_{, i}}\right)_{, i}-\frac{\partial W}{\partial \theta}+\Gamma_{i}^{v i s} \frac{\partial n_{i}}{\partial \theta}=0
\end{gathered}
$$

where $\rho$ is the density of the NLC, $\vec{F}$ is the external force (body force) per unit mass. The above equations assume homogeneous incompressibility of the nematic $(\nabla \rho=0)$ and negligible inertial effects. Furthermore, $\tilde{p}=p+W$, is a modified pressure term which accounts for the orientational elasticity, $W$ being the elastic energy density:

$$
2 W=K_{11}(\nabla \cdot \vec{n})^{2}+K_{22}[\vec{n} \cdot(\nabla \times \vec{n})]^{2}+K_{33}[\vec{n} \times(\nabla \times \vec{n})]^{2}+\text { mixed elastic terms (optional) }
$$

The constitutive laws for viscous torque, $\Gamma^{\mathbf{v i s}}$, and the stress tensor $\tilde{\boldsymbol{\sigma}}$ are respectively given by: 

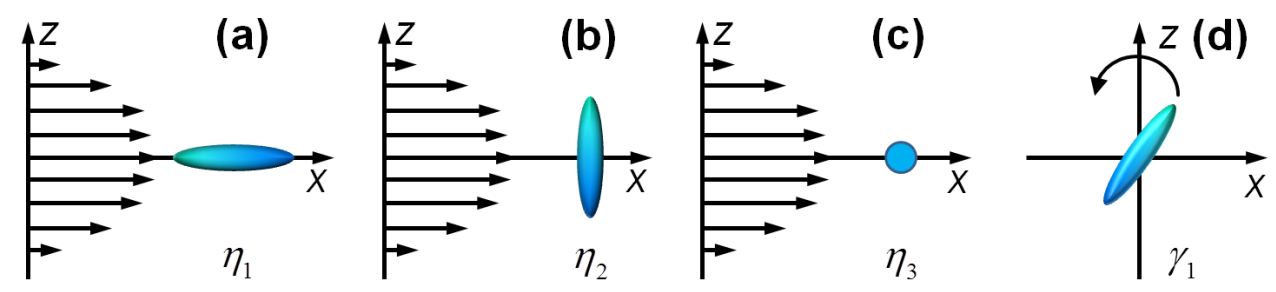

Figure 2.13: Viscosities in nematodynamics. $\frac{1}{2}\left(-\alpha_{2}+\alpha_{4}+\alpha_{5}\right)$, (c) $\eta_{3}=\frac{1}{2} \alpha_{4}$, and (d) rotational viscosity, $\gamma_{1}=\alpha_{3}-\alpha_{2}$.

$$
\begin{gathered}
\Gamma_{i}^{v i s}=-\left(\gamma_{1} N_{i}+\gamma_{2} A_{i, j} n_{j}\right), \text { and } \\
\tilde{\sigma}_{i, j}=\alpha_{1}\left(n_{k} A_{k l} n_{l}\right) n_{i} n_{j}+\alpha_{2} n_{j} N_{i}+\alpha_{3} n_{i} N_{j}+\alpha_{4} A_{i j}+\alpha_{5} n_{j} n_{k} A_{k i}+\alpha_{6} n_{i} n_{k} A_{k j}
\end{gathered}
$$

Here, $\gamma_{1}=\alpha_{3}-\alpha_{2}$ and $\gamma_{2}=\alpha_{6}-\alpha_{5}$ denote the rotational viscosity and the torsional coefficient, respectively. Additionally,

$$
A_{i, j}=\frac{1}{2}\left(\frac{\partial v_{i}}{\partial x_{j}}+\frac{\partial v_{j}}{\partial x_{i}}\right)
$$

denotes the components of the symmetric part of the shear rate tensor analogous to isotropic fluids. The angular velocity characterizing the director rotation relative to the fluid is given by $\vec{N}$ as under:

$$
\begin{aligned}
\vec{N} & =\frac{\mathrm{D} \vec{n}}{\mathrm{Dt}}-\vec{\Omega} \times \vec{n} \\
\text { with, } \Omega_{i, j} & =\frac{1}{2}\left(\frac{\partial v_{i}}{\partial x_{j}}-\frac{\partial v_{j}}{\partial x_{i}}\right)=\vec{\omega} / 2
\end{aligned}
$$

Here, $\vec{\Omega}$ and $\vec{\omega}$ vectors respectively denote the local rotation rate and the vorticity. The constants $\alpha_{1}$ to $\alpha_{6}$ are known as the Leslie coefficients, which have the dimensions of dynamic viscosity. The six Leslie coefficients however reduce to five independent values by considering the Parodi relation [16]:

$$
\alpha_{2}+\alpha_{3}=\alpha_{6}-\alpha_{5}
$$


The linear combinations of the Leslie coefficients defining the three Miesowicz viscosities (Fig. 2.13) are summarized through the following equations:

$$
\begin{aligned}
& \eta_{1}=\frac{1}{2}\left(\alpha_{3}+\alpha_{4}+\alpha_{6}\right), \\
& \eta_{2}=\frac{1}{2}\left(-\alpha_{2}+\alpha_{4}+\alpha_{5}\right), \text { and } \\
& \eta_{3}=\frac{1}{2} \alpha_{4}
\end{aligned}
$$

In fact, the Leslie coefficient $\alpha_{4}$ corresponds to the usual viscosity of an isotropic fluid.

\subsubsection{Poiseuille flow of nematic liquid crystals}

Nematic liquid crystals are classified into two categories with respect to their nematodynamic behaviour: (i) flow-aligning, for which $\alpha_{2} \alpha_{3}>0$, and (ii) tumbling nematic LCs, in which $\alpha_{2} \alpha_{3}<0$. We shall focus on NLCs of the flow-aligning kind.

Flow of nematic liquid crystals generally involves three different competing contributions namely, inertial, viscous and the elastic effects. In isotropic fluids the hydrodynamic stabilty is decided by the competing inertial and viscous effects, characterized by the Reynolds number:

$$
R e=\frac{\text { inertial effects }}{\text { viscous effects }}=\frac{\rho v L}{\mu}=\frac{v L}{v}
$$

The hydrodynamic instability manifests at high Reynolds numbers, typically occuring at high flow speeds, $v$ and low dynamic viscosities, $\mu$. The factor $L$ represents the characteristic lenght scale of the system, generally the hydraulic diameter [113]. In contrast to this, NLC flows show significant instabilities even at very low $R e \ll 1$. Nematodynamic instabilities occur primarily due to the contribution of the elastic effects. The flow stability in this case is characterized by the Ericksen number:

$$
E r=\frac{\text { viscous effects }}{\text { elastic effects }}=\frac{\mu \nu L}{K}=\frac{\nu L}{D}
$$

where, $D=K / \mu \approx 10^{-9} \mathrm{~m}^{2} / \mathrm{s}$ defines the diffusion constant for the director reorientation [76] as a function of the elastic constant $K$ and dynamic viscosity $\mu$. Clearly, the diffusion of vorticity is much more rapid than that of the director, $v \gg D$. Thus, even when a flow is hydrodynamically laminar, elastic effects may render the flow unstable. The loss of flow stabil- 
ity within microfluidic confinements is consequently characterized by the Ericksen numbers, rather than by the low $R e \approx 10^{-4}$.

In the case of flow-aligning NLCs, there exists specific director orientation in the shear plane at which the viscous torque on the director vanishes. If the director is oriented at an angle $\theta$ with the velocity, the viscous torque given by equation 2.40 yields:

$$
\Gamma_{z}^{v i s}=\left(\alpha_{2} \sin ^{2} \theta-\alpha_{3} \cos ^{2} \theta\right) \frac{\partial v}{\partial z} z
$$

which gives two solutions, stable and unstable, for $\theta$ as under:

$$
\begin{aligned}
\theta_{\text {stable }} & =+\tan ^{-1} \sqrt{\alpha_{3} / \alpha_{2}} \text { and } \\
\theta_{\text {unstable }} & =-\tan ^{-1} \sqrt{\alpha_{3} / \alpha_{2}}
\end{aligned}
$$

for $\alpha_{2} \alpha_{3}>0$ only [76]. Clearly, for $\alpha_{2} \alpha_{3}<0$, the solutions always lead to instability (tumbling nematics).

The anisotropy of the nematic viscosity leads to distinct effects in Poiseuille flow, with no isotropic analogy. One such effect is the generation of a transverse viscous stress [17, 23] when the director is held at an angle $\phi$ to the shear plane, shown in Fig. 2.14a. The director is then represented as $\vec{n}=(\cos \phi, \sin \phi, 0)$. Consequently,

$$
\begin{aligned}
\tilde{\sigma}_{x, z} & =\eta_{e f f} \frac{\partial v}{\partial z} \text { where } \\
\eta_{e f f} & =\eta_{1} \cos ^{2} \phi+\eta_{3} \sin ^{2} \phi
\end{aligned}
$$

Similarly,

$$
\tilde{\sigma}_{y, z}=\left[\left(\eta_{3}-\eta_{1}\right) \cos \phi \sin \phi\right] \frac{\partial v}{\partial z}
$$

Additionally, employing the conservation of mass and momentum and the constitutive equation of the stress tensor, we can write, 

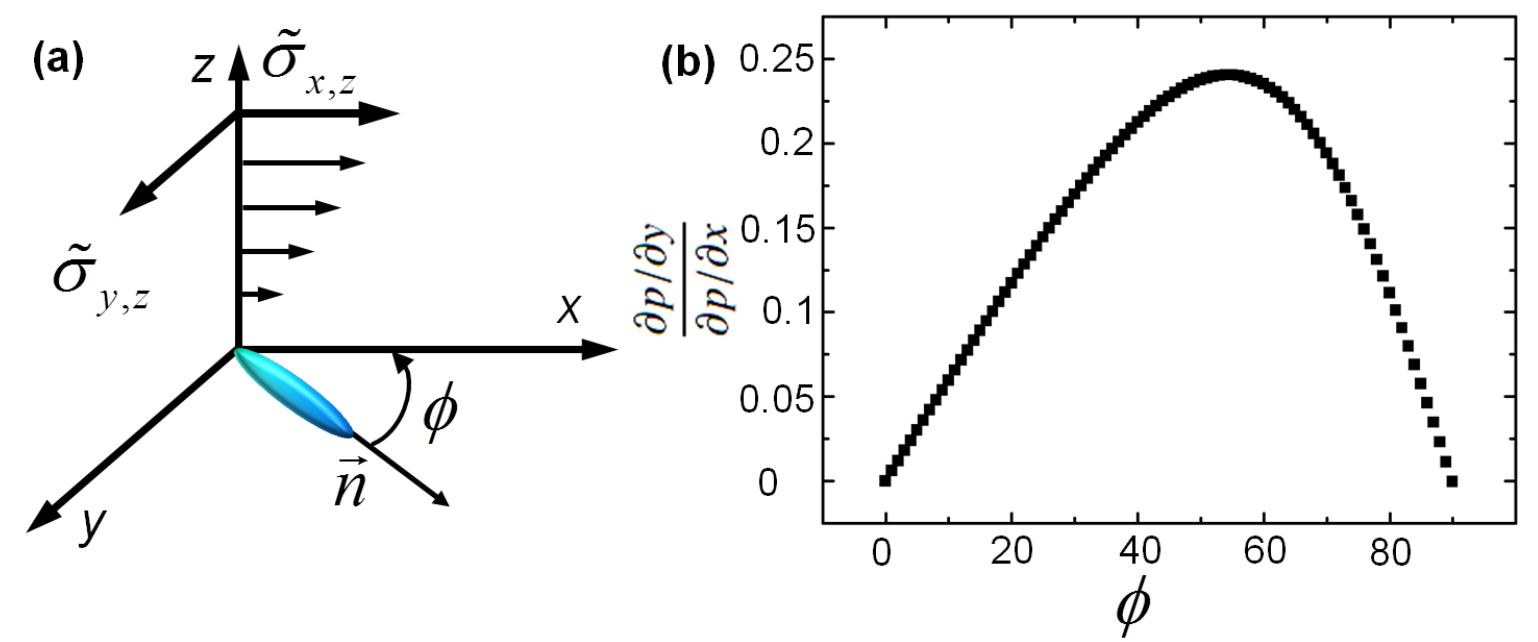

Figure 2.14: Transverse pressure gradient in Poiseuille flow of nematic LCs. (a) The schematic representation shows the orientation of the director relative to the flow field. The director was held fixed using a strong magnetic field. (b) Variation of the transverse pressure gradient normalized with the primary pressure gradient plotted as a function of $\phi$. For 5CB, $\eta_{1}=0.0204$ Pas and $\eta_{3}=0.0326$ Pas were considered.

$$
\begin{aligned}
& 0=-\frac{\partial p}{\partial x}+\eta_{e f f} \frac{\partial^{2} v}{\partial z^{2}} \text { and } \\
& 0=-\frac{\partial p}{\partial y}+\left(\eta_{3}-\eta_{1}\right) \cos \phi \sin \phi \frac{\partial^{2} v}{\partial z^{2}}
\end{aligned}
$$

By eliminating $\partial^{2} v / \partial z^{2}$, we obtain the ratio between the transverse and longitudinal pressure gradients:

$$
\frac{\partial p / \partial y}{\partial p / \partial x}=\frac{\left(\eta_{3}-\eta_{1}\right) \cos \phi \sin \phi}{\eta_{1} \cos ^{2} \phi+\eta_{3} \sin ^{2} \phi}
$$

Figure $2.14 \mathrm{~b}$ shows the variation of this pressure gradient ratio as a function of the angle $\phi$ for 5CB in nematic phase. The transverse pressure gradient has a maximum of $24 \%$ of the primary pressure gradient at around $\phi=54$ degrees. 


\subsubsection{Topological defects in flow}

Topological defects and flow interact with each other due to the coupling between the flow and the director field. This interaction is manifested usually in two forms: (i) Influence of topological defects on the flow field and vice versa [98], and (ii) possible generation of flow due to the interaction between topological defects [114, 115].

To begin with, we shall look into the average friction force experienced by a disclination moving through a nematic sample. For reasons of clarity, the estimations will be done assuming the one elastic constant approximation and the absence of any backflow effects. Let us consider a disclination line of rank $s$, parallel to the $z$ axis moving with a speed $v$ along $x$ direction. In the stationary regime, $\vec{n}(X, Y, t)=\vec{n}(x-v t, y, t)$, such that $X=x-v t$ and $Y=y$. Then, the energy dissipated in the system per unit time is given in terms of the rotational viscosity, $\gamma_{1}$ as:

$$
\begin{aligned}
E_{\text {diss }} & =\gamma_{1} \int\left(\vec{n} \times \frac{\partial \vec{n}}{\partial t}\right)^{2} \mathrm{~d} x \mathrm{~d} y \\
& =\gamma_{1} v^{2} \int\left(\frac{\partial \vec{n}}{\partial X}\right)^{2} \mathrm{~d} X \mathrm{~d} Y \\
& =\gamma_{1} v^{2} \int\left(\frac{\partial \varphi}{\partial X}\right)^{2} \mathrm{~d} X \mathrm{~d} Y
\end{aligned}
$$

where, $\varphi$ is the angle made by the director with $X$ axis, which is obtained from the balance of the viscous and elastic torques:

$$
\begin{aligned}
\Gamma^{v i s} & =\Gamma^{\text {elastic }} \\
\gamma_{1} v \frac{\partial \varphi}{\partial X} & =K \Delta \varphi
\end{aligned}
$$

By normalizing the lengths with the core radius, $r_{c}$, one obtains:

$$
\begin{gathered}
X^{*}=X / r_{c}, Y^{*}=Y / r_{c}, \text { and } \\
E r=\gamma_{1} v r_{c} / K
\end{gathered}
$$


Consequently,

$$
\Delta \varphi=\operatorname{Er} \frac{\partial \varphi}{\partial X^{*}}
$$

Hence, the dissipation outside the core radius can be calculated by integrating equation 2.62 over the limits $\left(r_{c}, \infty\right)$, yielding a dependence on the $E r$ as:

$$
E_{\text {diss }}=\gamma_{1} v^{2} f(E r),
$$

where $f(E r)$ is defined by the relation given by Ryskin and Kremenetsky as a function of the topological rank, $s$ [116],

$$
f(E r)=\pi s^{2} \ln \left(\frac{3.6}{E r}\right)
$$

From the dissipation theorem in stationary regime, $E_{\text {diss }}=-\vec{F}_{v i s} \cdot \vec{v}$, the friction force acting on the disclination is given by:

$$
\vec{F}_{v i s}=-\pi \gamma_{1} s^{2} \ln \left(\frac{3.6 K}{\gamma_{1} v r_{c}}\right) \vec{v}
$$

Additionally, the total dissipation can be approximated further in the limits of infinite sample size and small Ericksen numbers as:

$$
E_{\text {diss }}=\pi \gamma_{1} v^{2} s^{2} \ln \left(\frac{1}{E r}\right)
$$

Recent numerical investigations have revealed that the coupling between the director and the velocity fields has significant influence on the overall motion of topological defects. In particular, the defect speed is dependent not only on the topological strength, but also on the sense of rotation of the director about the core [114]. Figure 2.15a, b show the development of director and flow fields around two oppositely charged topological defects undergoing annihilation. The flow-director coupling was found to be dependent on the nature of the defect. While the speed of the $+1 / 2$ defect changed by $\approx 100 \%$ due to the backflow effect, on the $-1 / 2$ defect, the influence of backflow was marginal. As is seen clearly in Fig. 2.15k, d, the speed of the positive defect is significantly higher than the negative one, especially at small separations. Furthermore, Svenšek and Žumer showed that the backflow mechanism is responsible for changing the director relaxation completely [117]. Subsequently, the numerical results were verified experimentally for semi-integer [60] and integer [118, 119] defect pairs. 


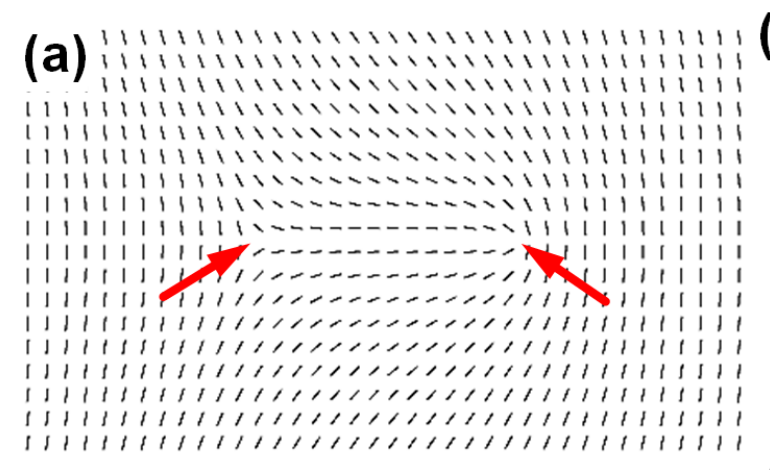

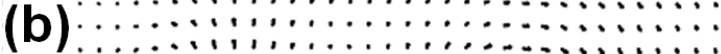
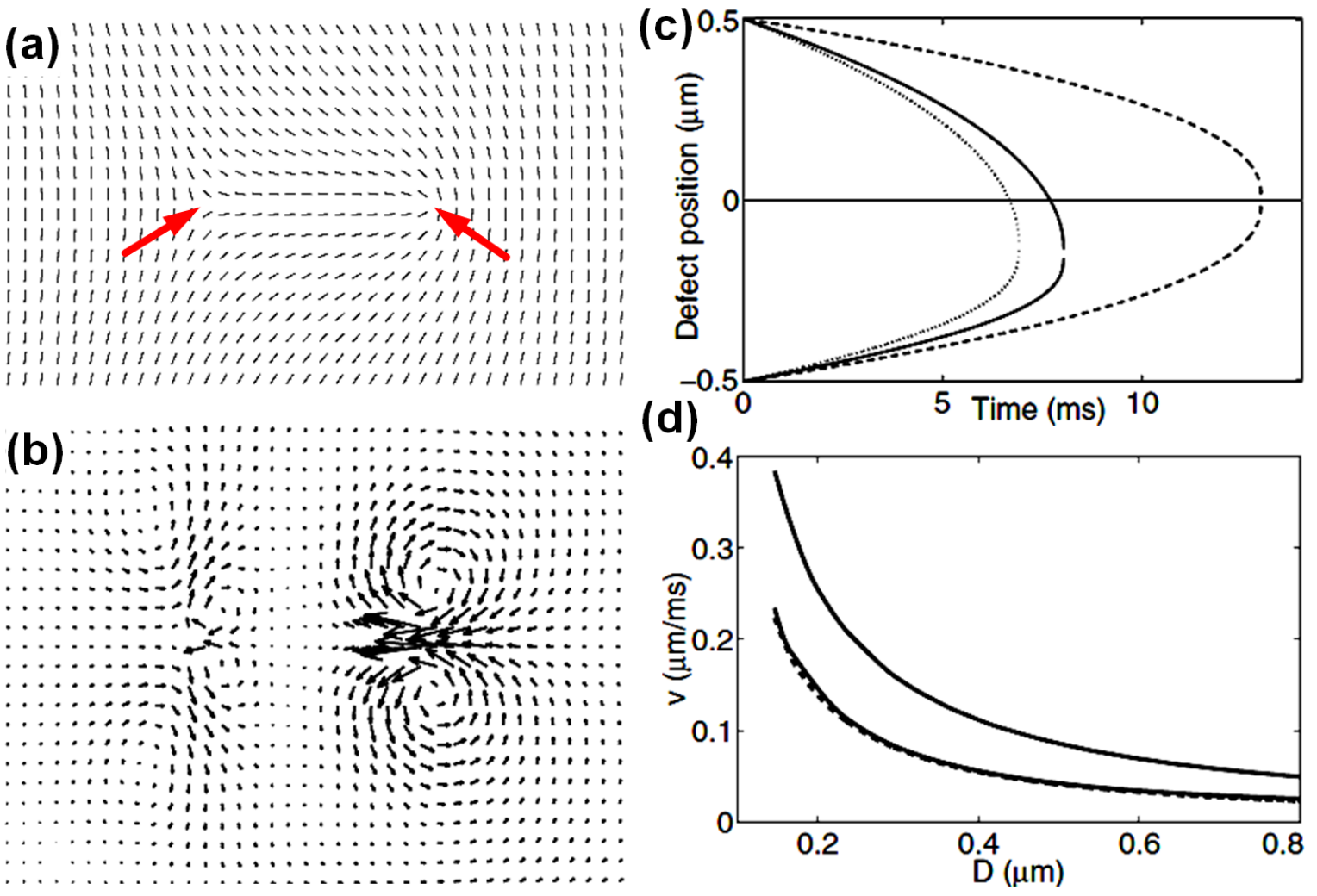

Figure 2.15: Effect of backflow on defect speed. (a) Director field of a pair of topological defects undergoing annihilation. The left and right arrows indicate $-1 / 2$ and $+1 / 2$ defects respectively. (b) Velocity field corresponding to the defect pair. (c) Time evolution of the defect position. The lower and upper curves correspond to $-1 / 2$ and $+1 / 2$ defects respectively. (d) Speed of the defects as a function of separation between them. The $+1 / 2$ defect travels faster than the $-1 / 2$ defect. Reproduced from [114]. 


\subsection{Nematic colloids}

Nematic colloids can be broadly defined as the dispersion of minute micrometer sized particles (colloid particles) in a continuous phase consisting of a nematic liquid crystal. The interactions between nematic colloids stem from the long-range orientational ordering [120], which is disturbed due to the presence of the inclusions. In contrast to the colloidal interactions in an isotropic fluid (typically van der Waals type), the interaction between nematic colloids are much stronger - of the order of $1000 k_{B} T$ - originating from the nematic elasticity [121]. Furthermore, the magnitude of the nematic interaction can be varied by tuning the surface properties of the colloids. Different boundary conditions at the colloid-nematic interface lead to different orientations of the director at the surface [122]. This results in a wide variety of phenomena [25], which has consequently lead to novel routes to self-assembled structures [123-128].
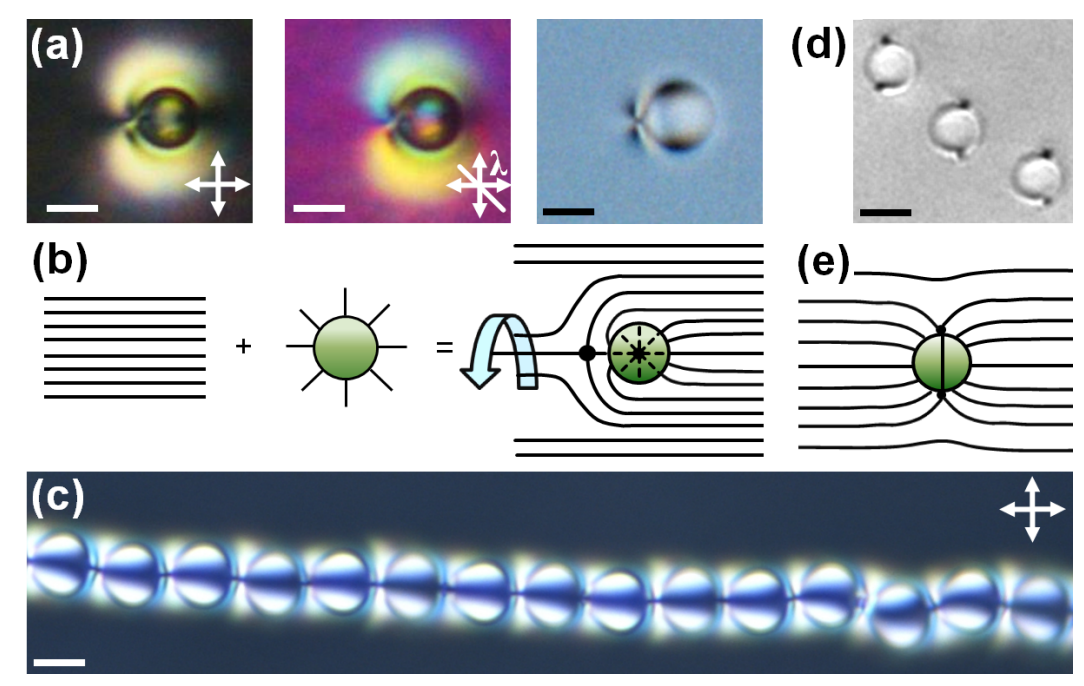

Figure 2.16: Nematic colloids with homeotropic surface anchoring. (a) Point-like hedgehog defect residing on a nematic colloid. From left to right, the micrographs were taken between crossed polarizers, crossed polarizers with $\lambda$ retardation plate, and in white light. (b) Schematic representation of the director field. (c) Colloidal chain formed due to self-assembly of dipolar structures. (d) Nematic colloid of quadrupolar symmetry observed in white light. (e) Schematic of the director field representing a Saturn-ring defect. Scale bar in the micrographs correspond to $5 \mu \mathrm{m}$.

Interestingly, nematic colloids or rather emulsions of water droplets, were first employed for revealing the nematic director field [129]. The droplets self-assembled into chains and ori- 
ented parallel or oblique to the director field $\vec{n}$. The self-assembly of droplets was investigated in details by Poulin and co-workers [24]. It was made evident that the inherent anisotropy of the host was responsible for such interactions. Coupled with the effects of the boundary conditions existing on the particles, it was found that the topological constraints due to the particles led to different types of interactions [121].

Particles possessing homeotropic anchoring yields two types of topological defects, as shown in Fig. 2.16, Figure 2.16a shows a particle accompanied by a point-like defect. The presence of the homeotropic anchoring on the particle induces strong distortions in the director field close to the surface resulting in the generation of the point defect. The colloid and its accompanying defect resemble an electric dipole due to the director field around them. The director field around the particle was resolved by polarization microscopy. As shown in Fig. 2.16 , the dipole comprises the point-like defect, known as hyperbolic hedgehog defect [25], and the colloid particle itself, which behaves as a source of an orientational field, a radial hedgehog defect. Topologically, hyperbolic and radial hedgehogs correspond to -1 and +1 defects, respectively. Far away from the particle, the director field is free of distortions, determined simply by the surface properties of the confinement.

However, within thin nematic samples, the dipolar field around the particles is strongly influenced by the confining surfaces [121]. Strong confinement effects may ultimately split up the hyperbolic hedgehog into a defect loop encircling the particle [25, 130, 131], as shown in Fig. 2.16d. The defect loop, also referred to as the Saturn-ring defect, is a topological equivalent of the electrical quadrupole (Fig. 2.16e). Nematic colloids of dipolar and quadrupolar symmetries interact to form long chains, as shown in Fig. 2.16.

As a consequence of long-range ordering, the effects of the elastic distortions are propagated through distances much larger than the characteristic length of the system. As a result, the interaction between nematic colloids is also termed as structural force. Structural forces observed in nematic systems are significantly anisotropic. In close proximity, the director distortions of the particles overlap, leading to repulsive or attractive interaction potentials [132]. Depending upon the relative orientation of the dipolar and quadrupolar structures [133], a wide gamut of colloidal assemblies in $1 D, 2 D$, and $3 D$ can be realized (Fig. 2.17).

It may be reasonable to assume here that such elastcity-mediated interactions also hold good for a nematic colloid particle in the vicinity of a disclination line. Indeed, it has been shown by Galerne and co-workers, that colloidal particles show anisotropic attractive or repulsive forces in the vicinity of a defect line. Please refer to [126, 134] for futher details. 

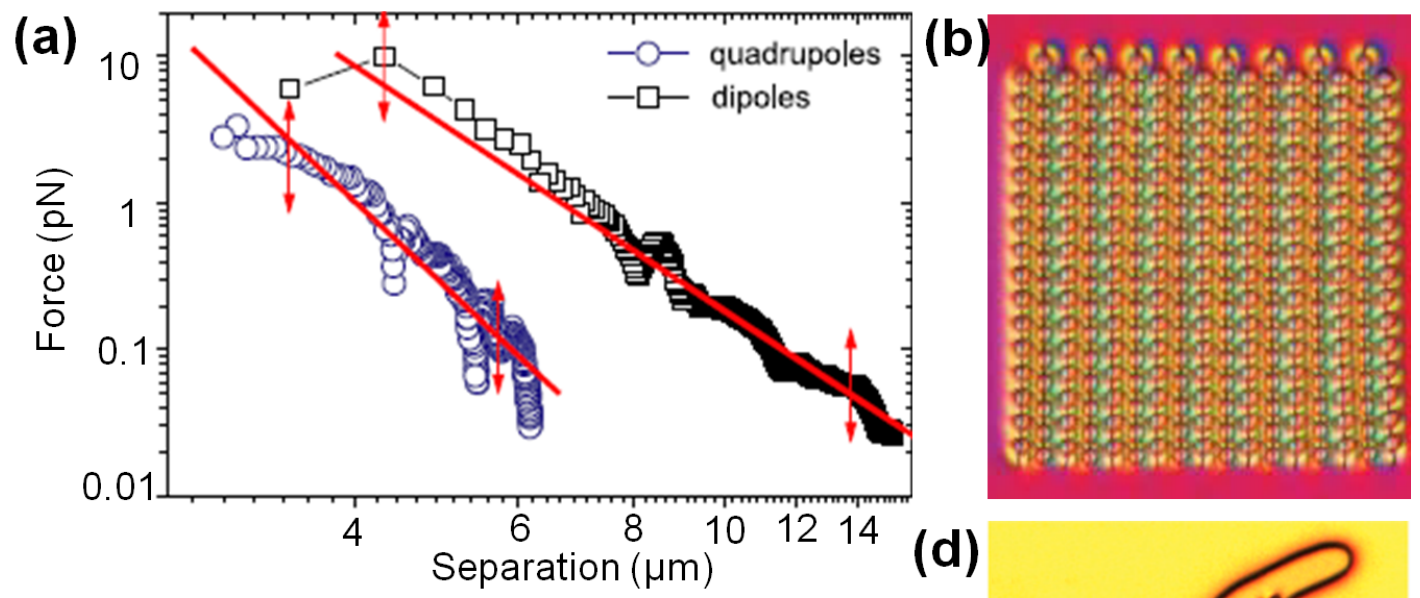

(C)
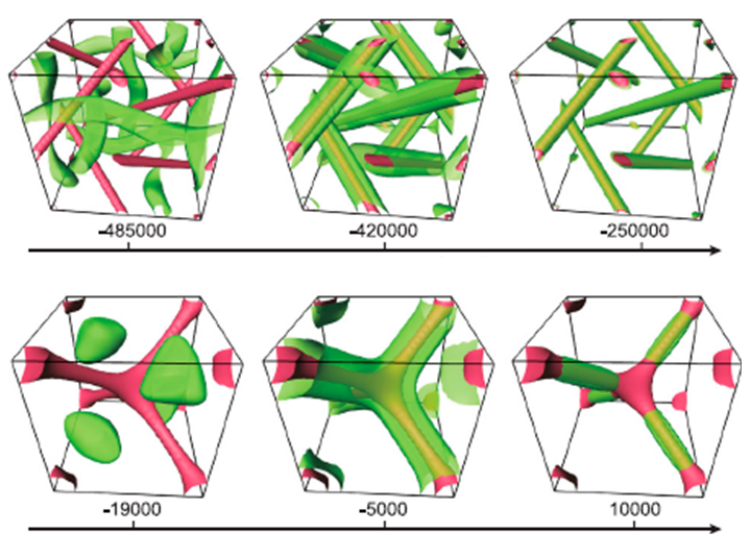

Free energy density $\left(\mathrm{k}_{\mathrm{B}} T / \mu \mathrm{m}^{3}\right)$

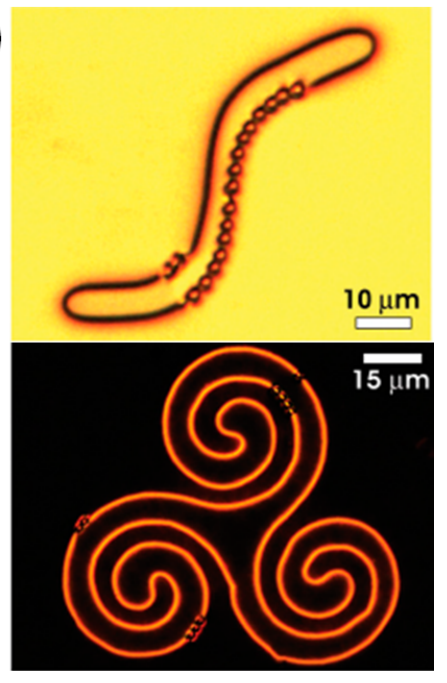

Figure 2.17: Self-assembled structures in nematic colloids. (a) Pair-interaction force of dipolar and quadrupolar particles as a function of separation. Measurements by Muševič et al. [133]. (b) Self-assembled 2D crystal. Adapted from [121]. (c) Defect-mediated 3D colloidal crystal in exotic blue phase, demonstrated by Ravnik et al. [128]. Note the exceptionally high free energy density values. (d) Demonstration of colloid trapping in optoelastic traps developed by Smalyukh and co-workers [135]. 


\section{Chapter 3}

\section{Materials and experimental methods}

This chapter focuses on the materials and the experimental methods employed for this work. In the context of materials, the properties relevant to the current work are emphasized. The list of materials broadly includes nematic liquid crystals, colloidal particles, and the fluorescent dyes. Additionally, reagents for generating specific surface attributes on colloidal particles and water droplets will be presented here. The experimental methods involve multiple components: microfluidic device and flow set up, flow and surface characterization techniques, and velocity measurement tools. Functionalization of microchannels, involving a combination different physico-chemical methods, will be dealt in a separate chapter.

\subsection{Nematic liquid crystal}

Throughout this study, the single component thermotropic nematic liquid crystal 4-cyano4'-pentyl-1,1'-biphenyl was used (Fig. 2.1). The compound is commonly known as 5CB or $\mathrm{K} 15$, and constitutes a primary component of most of the LC mixtures used for commercial purposes. 5CB was procured from Synthon Chemicals (Germany), and used without additional purification. The molecule consists of two consecutively bonded phenyl rings attached to a nitrile group (CN) on one end and a five-carbon alkyl chain $\left(\mathrm{C}_{5} \mathrm{H}_{11}\right)$ on the other end. While the former renders the molecule strongly polar, the latter contributes in making it highly anisotropic in shape. With respect to LC properties, $5 \mathrm{CB}$ is uniaxial and exhibits only the nematic phase at room temperatures. At $T_{N I} \approx 34.5^{\circ} \mathrm{C}, 5 \mathrm{CB}$ undergoes a transition to the isotropic phase. The milky appearance of $5 \mathrm{CB}$ changes to that of a clear one during the nematic-to-isotropic transition. Below $T_{X N} \approx 22.4{ }^{\circ} \mathrm{C}, 5 \mathrm{CB}$ crystallizes to solid state. Higher 
homologues of 5CB can however differ in their phase behaviour: While 8CB (containing an eight-carbon alkyl chain) shows both nematic and smectic phases, 10CB (ten carbons in the alkyl chain) exhibits only smectic, but no nematic phase. An exhaustive list of material properties of different homologues of 5CB can be found in [136]. The choice of the nematic liquid crystal, 5CB as the flowing matrix was motivated due to the following reasons:

i) $5 \mathrm{CB}$ is in nematic phase at usual room-temperatures,

ii) lack of positional ordering in nematics makes them suitable for flow experiments, and

iii) $5 \mathrm{CB}$ is stable within microfluidic confinements fabricated using soft lithography.

Tables 3.1, 3.2, 3.3, and 3.4 enlist values of different physical properties of 5CB. The values have been adapted from references [75, 136, 137]. Various methods which have been applied to estimate these values are discussed in [138-140].

\begin{tabular}{|c||c||c|c||c|c|}
\hline $\begin{array}{c}\mathrm{M} \\
(\mathrm{g} / \mathrm{mol})\end{array}$ & $\begin{array}{c}\rho(I) \\
\left(\mathrm{kg} / \mathrm{m}^{3}\right)\end{array}$ & $\begin{array}{c}T_{X N} \\
\left({ }^{\circ} \mathrm{C}\right)\end{array}$ & $\begin{array}{c}T_{N I} \\
\left({ }^{\circ} \mathrm{C}\right)\end{array}$ & $\begin{array}{c}\Delta \mathrm{H}_{X N} \\
\left(10^{6} \mathrm{~J} / \mathrm{m}^{3}\right)\end{array}$ & $\begin{array}{c}\Delta \mathrm{H}_{N I} \\
\left(10^{6} \mathrm{~J} / \mathrm{m}^{3}\right)\end{array}$ \\
\hline \hline 249.359 & 1002.4 & 22.4 & 34.5 & 68.5 & 1.56 \\
\hline
\end{tabular}

Table 3.1: Thermodynamic properties of 5CB.

\begin{tabular}{|c||c|c|c|c||c|c|c|}
\hline $\begin{array}{c}T \\
\left({ }^{\circ} \mathrm{C}\right)\end{array}$ & $\begin{array}{c}\eta_{1} \\
(\mathrm{mPas})\end{array}$ & $\begin{array}{c}\eta_{2} \\
(\mathrm{mPas})\end{array}$ & $\begin{array}{c}\eta_{3} \\
(\mathrm{mPas})\end{array}$ & $\begin{array}{c}\gamma_{1} \\
(\mathrm{mPas})\end{array}$ & $\begin{array}{c}K_{11} \\
(\mathrm{pN})\end{array}$ & $\begin{array}{c}K_{22} \\
(\mathrm{pN})\end{array}$ & $\begin{array}{c}K_{33} \\
(\mathrm{pN})\end{array}$ \\
\hline \hline $25^{\circ} \mathrm{C}$ & 22 & 123 & 43 & 77 & 6.4 & 3.2 & 9 \\
\hline $36^{\circ} \mathrm{C}$ & 26 & 26 & 26 & - & 4.9 & 2 & 4 \\
\hline
\end{tabular}

Table 3.2: Viscosity coefficients and elastic constants of 5CB at nematic and isotropic phases.

\begin{tabular}{|c||c|c|c|c|c|c|}
\hline $\begin{array}{c}T \\
\left({ }^{\circ} \mathrm{C}\right)\end{array}$ & $\begin{array}{c}\alpha_{1} \\
(\mathrm{mPas})\end{array}$ & $\begin{array}{c}\alpha_{2} \\
(\mathrm{mPas})\end{array}$ & $\begin{array}{c}\alpha_{3} \\
(\mathrm{mPas})\end{array}$ & $\begin{array}{c}\alpha_{4} \\
(\mathrm{mPas})\end{array}$ & $\begin{array}{c}\alpha_{5} \\
(\mathrm{mPas})\end{array}$ & $\begin{array}{c}\alpha_{6} \\
(\mathrm{mPas})\end{array}$ \\
\hline \hline $26{ }^{\circ} \mathrm{C}$ & -6 & -81.2 & -3.6 & 65.2 & 64 & -20.8 \\
\hline
\end{tabular}

Table 3.3: Leslie coefficients of 5CB.

The stability of a nematic material with respect to the microchannel surfaces - glass and a transparent polymer mould - deserves a mention here. Although glass as a material is chemically stable towards LCs, the same might not be true for the polymer mould onto which 


\begin{tabular}{|c||c|c|c|}
\hline$T\left({ }^{\circ} \mathrm{C}\right)$ & $n_{\|}$ & $n_{\perp}$ & $\Delta n$ \\
\hline \hline $25{ }^{\circ} \mathrm{C}$ & 1.727 & 1.539 & 0.188 \\
\hline $36{ }^{\circ} \mathrm{C}$ & 1.65 & 1.65 & 0 \\
\hline
\end{tabular}

Table 3.4: Refractive indices and optical birefringence of 5CB at nematic and isotropic phases. The values correspond to $\lambda=546.1 \mathrm{~nm}$.

microchannels are cast. Due care was taken that the nematic 5CB did not react with the microchannel surfaces (3.3.1), either due to physcial absorption, or due to chemical interactions. While 5CB was found to be very stable within microfluidic devices, a commercially available NLC mixture, ZLI-4792 (Merck KGaA, Germany), was found to be incompatible with the polymer material. Though the commercially available LC mixture offered interesting properties like low birefringence - suitable for confocal microscopy - it was not suited for long-term microfluidic experiments. In comparision to $5 \mathrm{CB}$, which was stable for months, ZLI-4792 degraded much more rapidly within the the PDMS-glass microchannels. This got clearly manifested through the nematic textures formed when the microchannels were filled by the two different materials. While 5CB textures were stable for days, the nematic textures due to ZLI-4792 varied drastically within hours. A possible reason for this might lie in certain chemical components of the mixture reacting preferentially with the polymer material (PDMS) used for fabricating the channels. Figure 3.1 shows a comparison between the fate of the textures formed by 5CB and ZLI-4792 within PDMS-glass microchannels, 24 hours after filling them up.
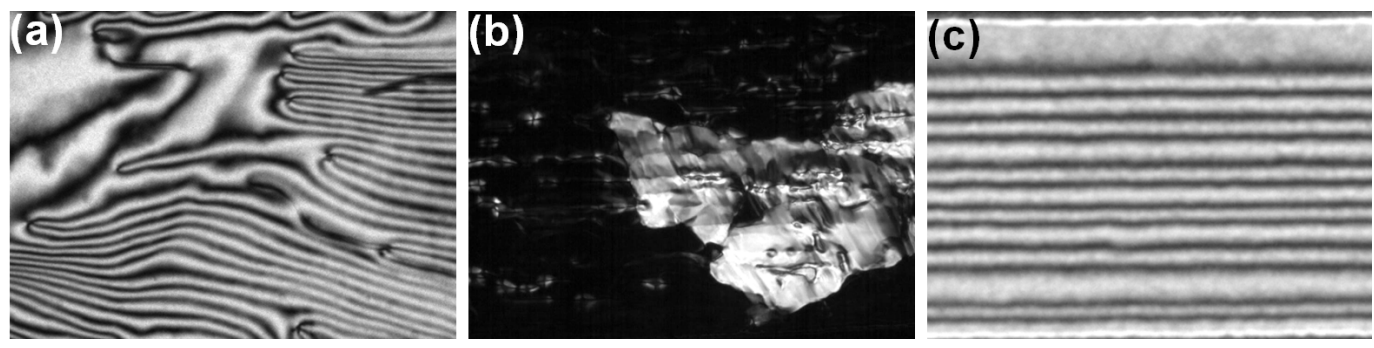

Figure 3.1: Material compatibility. (a) Nematic texture formed by ZLI-4792 within a glassPDMS microchannel possessing degenerate surface anchoring. (b) Degradation of the nematic texture after $\approx 12 \mathrm{~h}$ from (a). (c) Nematic texture formed by flow of 5CB. The texture was observed to stable for days. 


\subsection{Preparation of the nematic colloids}

Nematic colloidal dispersions were prepared using polystyrene (Licristar, Japan) and silica (microparticles $\mathrm{GmbH}$, Germany) beads. Although preliminary investigations were conducted using the polystyrene beads of mean diameter $\approx 4.5 \mu \mathrm{m}$, subsequently silica $\left(\mathrm{SiO}_{2}\right)$ were employed due to procurement reasons. The diameter of the silica particles was chosen between $2 \mu \mathrm{m}$ and $10 \mu \mathrm{m}$. Detailed material properties of the particles can be found in reference [141].

Prior to preparation of the nematic dispersion, the particles were surface-treated for inducing homeotropic anchoring of the 5CB molecules on them. A long-chain silane molecule, Octadecyldimethyl (3-trimethoxysilyl propyl) ammoniumchlorid, conc. 60\% in methanol (ABCR GmbH, Germany), commonly known as DMOAP, was used for this purpose (Fig. 3.2). A low concentration $(\approx 0.1-1 \%$ by weight) aqueous solution of DMOAP was first prepared, into which the dry colloidal particles were introduced. After about ten minutes, the aqueous dispersion was centrifuged for $60 \mathrm{~s}$ at $3000 \mathrm{rpm}$ and the DMOAP solution was decanted. Thereafter the particles were rinsed with millipore water followed by centrifuging. This step was repeated several times. The residual solvent was removed by thermal treatment in an oven at $80^{\circ} \mathrm{C}$ for $15 \mathrm{~min}$ and at $110^{\circ} \mathrm{C}$ for $1 \mathrm{~h}$. The overall treatment reduced the surface energy, rendering the particles hydrophobic. The particles were then suspended at low concentrations $\approx 1 \%$ by weight in isotropic $5 \mathrm{CB}$. The dispersion was sonicated and then allowed to cool down, resulting in a homogeneous dispersion colloidal particles in nematic 5CB. In general, DMOAP is expected to induce strong homeotropic anchoring of the LC molecules [58, 142]. In Chapter 4 we shall once again use DMOAP to induce homeotropic anchoring on the walls of the microchannels.

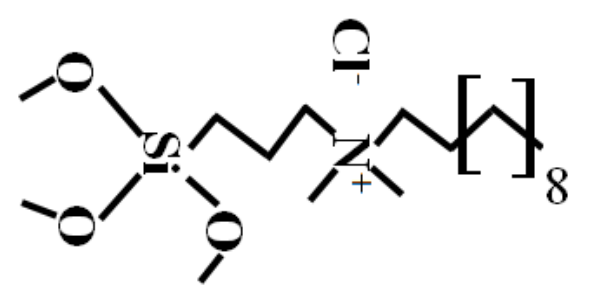

Figure 3.2: Octadecyldimethyl (3-trimethoxysilyl propyl) ammoniumchlorid (DMOAP) molecule.

Water droplets of sizes similar to the colloid particles were also dispersed within the nematic host. Generally, homeotropic anchoring at the water-LC interface is achieved using 
a suitable surfactant [143]. In this present study, aqueous solution of the surfactant CTAB (conc. below $C M C$ ) has been used. The surfactant-water solution (1\% by weight) was mixed with 5CB in isotropic phase and sonicated for ten minutes. The emulsion was then allowed to cool down to the nematic phase. This yielded polydispered water droplets with homeotropic anchoring at the water-5CB interface. Alternatively, existing ways of generating highly monodispersed droplets can be used [144].

\subsection{Microfluidic confinement and flow set-up}

The nematic liquid crystal and the nematic colloids were flowed through microchannels of different geometry and dimensions. The microchannels were fabricated using the techniques of soft lithography. Additionally, the walls of the channels were functionalized to induce different kinds of nematic anchoring, providing a wider platform to investigate nematic micro-flows. While most of the investigations were done at a constant volume flow rate, $Q$, some experiments also employed a constant pressure gradient along the channel to create the nematic flow. The director dynamics were studied using a combination of polarization and fluorescence confocal microscopy techniques. The measurements of flow speeds were carried out either by tracking tracer particles over time, or using the technique of dual-focus fluorescence correlation spectroscopy.

\subsubsection{Fabrication of microfluidic devices}

Microfluidic devices were fabricated using the techniques of soft photolithography [145]. Photolithography is a standard method employed to pattern silicon substrates. Figure 3.3 shows the steps involved in the fabrication process schematically. The microfluidic devices were fabricated within an in-house clean room facility. A photo-sensitive material called photoresist is first spin-coated on a silicon substrate and baked at moderate temperatures to drive away traces of solvent, see Fig. 3.3 (i). The speed and duration of the spin-coating process dictates the thickness of deposited photoresist, which is effectively also the depth of the microchannels. A patterned photomask is then placed on the photoresist, followed by brief exposure to UV light, as depicted in Fig. 3.3 a(ii). In this present work, I have used a negative photoresist: Negative photoresists cross link post exposure. UV light thus cures the photroresist layer only at regions exposed to it. After this process, the coated silicon substrate is immersed and stirred in a suitable solvent, known as the developer. Portions of the pho- 
(a)

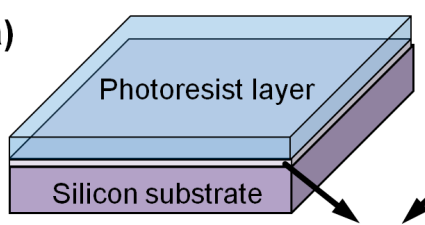

(i)

(b)

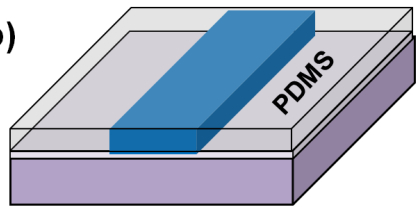

(i)

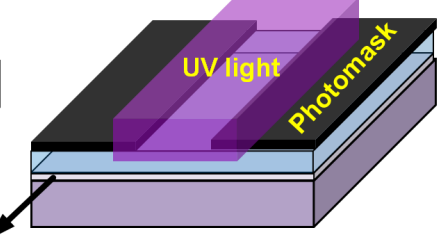

(ii)

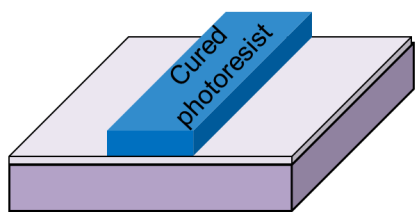

(iii)

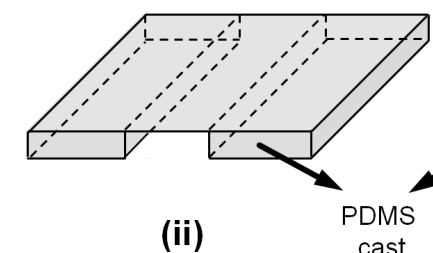

(ii)

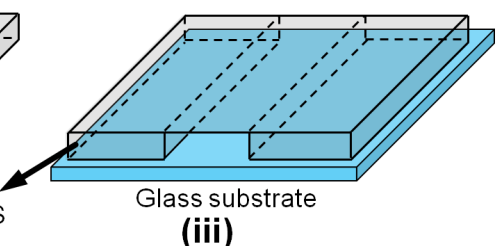

(iii)
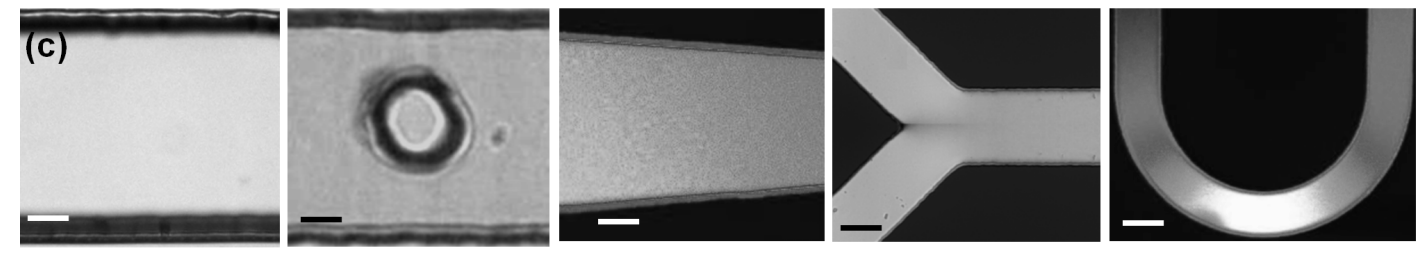

Figure 3.3: Fabrication of microfluidic devices. (a) Photolithography technique. (i) Deposition of photoresist on a silicon substrate. (ii) Exposure to UV light through the photomask. (iii) Post development, the UV-cured photoresist layer remains, whereas rest of the layer is washed away. (b) Preparation of PDMS cast. (i) PDMS and cross-linker mixture is poured over the silicon substrate with photoresist. (ii) The PDMS layer is thermally cured and pulled out from the silicon substrate, leaving behind the PDMS relief. (iii) Glass and PDMS are exposed to plasma and surface bonded, producing the PDMS-glass microchannel. (c) Microfluidic geometry. Optical micrographs of microchannel sections, from left to right: Linear, linear channel with micro-pillar, diverging, Y, and U shaped microchannels. Scale bar: $20 \mu \mathrm{m}$

toresist film which did not undergo cross-linking are washed away by the developer, leaving behind patterned features, Fig. 3.3a(iii).

Subsequently, PDMS reliefs were prepared following the standard soft lithography techniques [146]. Polydimethylsiloxane, PDMS (Sylgard 184, Dow Corning) was first mixed with a cross-linker in 10:1 proportion (by weight), and poured on the photoresist (Fig. $3.3 \mathrm{~b}(\mathrm{i})$ ). The moulded reliefs were obtained by pulling off the PDMS after thermal curing, see Fig. 3.3b(ii). Subsequently, the reliefs were bonded to glass substrates after being exposed to air/oxygen plasma, as shown in Fig. 3.3 p(iii). Different geometries - linear, U, T, L, and Y shaped, meandering, diverging, and channels with micro-pillars - were prepared using above techniques 
(Fig. 3.3 ). The channels had rectangular cross-section, with the channel depth $d$ varying between 5 and $100 \mu \mathrm{m}$, while the channel width $w$ was typically maintained between $50 \mu \mathrm{m}$ to $500 \mu \mathrm{m}$. The distance between the inlet and the outlet port was generally set to $20 \mathrm{~mm}$, defining the length $l$ of the channel. To ensure that the measurements were carried out in the fully developed flow regime, the length $l$ was maintained much longer than the entrance length - the minimum channel length required for the flow to be fully developed. No perceptible swelling of PDMS by 5CB was observed during the course of experiments. This is in agreement with the observed dependence of the material swelling ratio on the substance polarity $(\approx 4.8 \mathrm{D}$ for 5CB) [147].

\subsubsection{Flow setup}

At both ends of the microchannel, cylindrical holes with $750 \mu \mathrm{m}$ diameter were punched through the PDMS to provide housing for the flow tubings. Two teflon tubes with $300 \mu \mathrm{m}$ inner diameter and $760 \mu \mathrm{m}$ outer diameter were inserted into each of the housings and served as connectors for inlet-to-source and outlet-to-sink, respectively. Figure 3.4 shows such a microchannel. The housing diameter was kept smaller than that of the tubes to ensure good mechanical fitting and to avoid leakage.
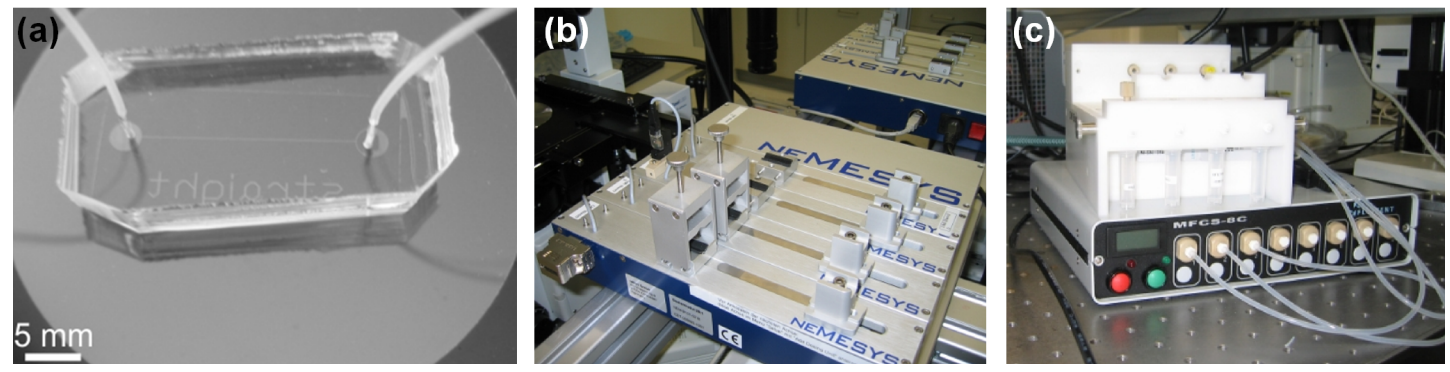

Figure 3.4: PDMS-glass microchannel and flow equipments. (a) Image shows a typical PDMS-glass microchannel. The teflon tubings inserted into the PDMS cast serve as the inlet and outlet connectors. (b) Pumps used for micro/nanoliter flows. (c) Equipment for constant pressure flows. Image courtesy for (b) and (c): J. C. Baret and group.

The inlet tube was connected to a gas-tight microlitre syringe (1001LT, Hamilton Bonaduz), which was driven by a gear pump (neMESYS, Cetoni) with a flow rate precision of nanoliter per hour, which is around $\pm 0.02 \%$ of the corresponding flow rates used. Alternatively, Poiseuille flow was created within the microchannels by applying a pressure difference be- 
tween the inlet and outlet ports using commercially available Fluigent Maesflow Flow Controller (France). The outlet pressure in the experiments was set to atmospheric pressure. In some cases, pressure-driven flow was created by using a gravitational head as well.

\subsubsection{Functionalization of microfluidic devices}

Microfluidic devices were functionalized for investigating nematic flows within microchannels possessing a variety of surface anchoring conditions. Specific anchoring states within the microfluidic devices were achieved using a combination of different physical and chemical methods. Due to a rather diverse set of steps involved, Chapter 4 is separately devoted towards studying the functionalization techniques (see (3) on the publication list).

\subsection{Characterization techniques}

Nematic micro-flows involve two separate aspects of characterization: director orientation, and flow speed measurement. While director orientation was resolved using polarization optical microscopy (POM) and fluorescence confocal polarization microscopy (FCPM), flow speed was measured by tracking the displacement of tracers over time, and by looking at po-

sitional correlations of dye molecules doped in the nematic 5CB. Generally, the director and the flow speed were resolved within the same microscopy setup.

\subsubsection{Polarization optical microscopy}

In Chapter 2.5.1, the concept of optical birefringence was introduced, and thereby the expression for the transmitted polarized light intensity was presented. Here, we shall look into the experimental aspects of polarization optical microscopy (POM). All experiments were conducted using transmission microscopy, i. e., the light passing through the sample was analyzed. Figure 3.5 a presents the overall experimental technique. Unpolarized white light from the microscope illumination source is first passed through a polarizer. A polarizer is an optical filter that converts unpolarized light and passes only a specific linear polarization decided by the polarizer axis (indicated by the double-headed arrow in Fig. 3.5a). The polarized light on reaching the NLC sample is split into the ordinary and extraordinary components as shown in Fig. 2.7. Consequently, an elliptically polarized light is obtained after the LC sample, which then passes through another polarizer, known as the analyzer, before reaching the microscope 

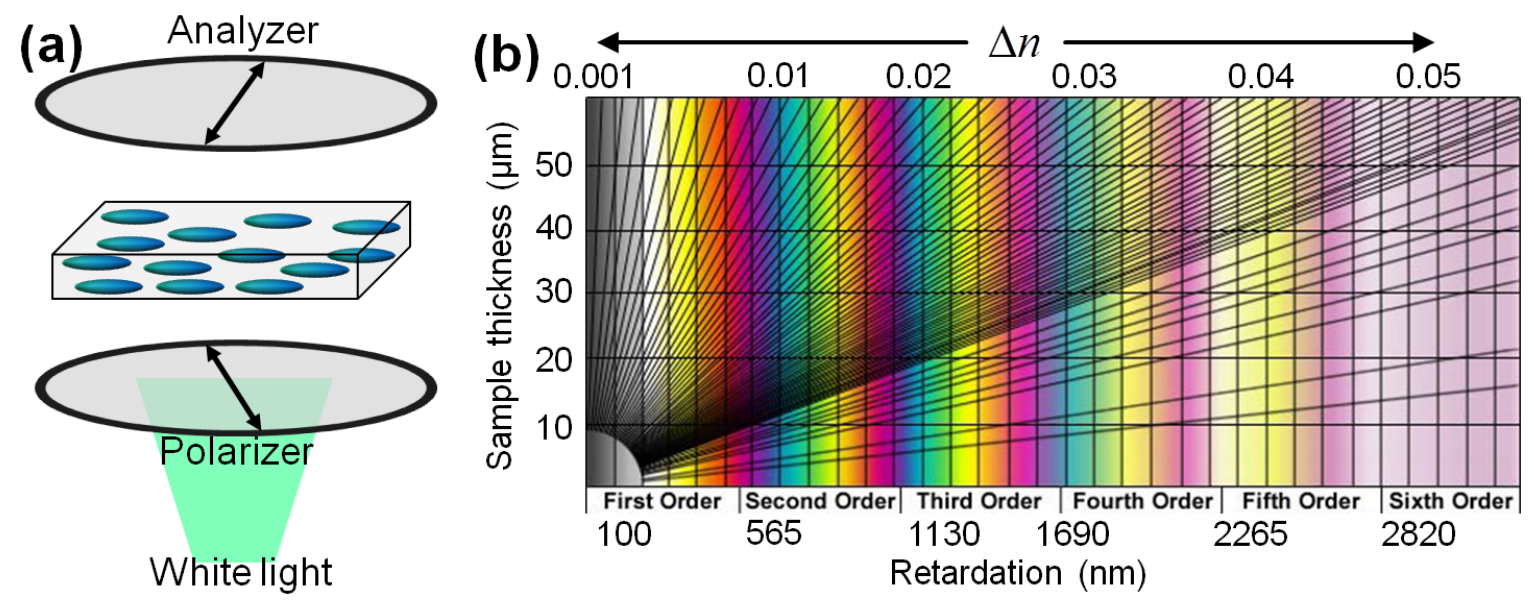

Figure 3.5: Polarization optical microscopy (POM). (a) Schematic representation of the POM principle. (b) Michel-Lévy birefringence chart [148].

ocular or the high-sensitivity CCD camera. In fact, the analyzer is also a polarizer, whose axis can be parallel, perpendicular, or at any intermediate angle relative to the polarizer. In the present setup, both the polarizer and the analyzer could be rotated with a precision of $\pi / 180=1^{\circ}$. When the polarizer and analyzer axes are mutually perpendicular it is termed as crossed polarized state. Additionally, by placing the microchannel on a rotating stage, the angle of the sample relative to the crossed polarizers was changed. When a component of the elliptically polarized light is transmitted through the crossed analyzer, the sample appears bright. No light is transmitted when the optical axis of the NLC is parallel to one of the polarizer axes. This renders a dark appearance to the sample when observed between the crossed polarizers.

The static and the flow-induced equilibrium director orientation of 5CB in the microchannels was determined using a Nikon Eclipse LV100 (Japan) polarizing microscope. Depending upon the region of interest and optical resolution desired, a set of different objectives was used: $5 \times, 10 \times, 20 \times, 40 \times, 60 \times$, and $100 \times$. Barring the $60 \times$ and $100 \times$ oil objectives, the rest were air objectives. The numerical aperture, NA, for lower magnification objectives was typically around 0.5 , while for the oil objectives the NA varied from 1.25 to 1.4. In addition to the orthoscopic studies, conoscopy [149] was performed by introducing a Bertrand lens between the analyzer and the ocular. Although conoscopy is a frequently used technique in the field of minerology, it is particularly helpful in the detection of the axiality (uniaxial or biaxial), and the optical characterization (optically positive or negative) of LC samples [72]. Besides 
complementing observations from the standard POM methods, the conoscopic technique is also useful in identifying the exact nature of the surface anchoring in the LC samples [150].

In the previous chapter, we have seen that the intensity of the polarized light after the analyzer depends upon the sample birefringence, thickness, and the orientation of the LC optical axis relative to the analyzer axis (see equation 2.30). When the sample is illuminated using white light, one observes a colourful birefringent texture, especially in thin samples. Michel Lévy, a French geologist, correlated the dependence of the birefringence (and its order) on the sample thickness and presented it using a chart, as shown in Fig. 3.5b.

In a homeotropic sample, the direction of the light propagation coincides with that of the optical axis, i. e. $\phi=0$, and from the equations 2.26 and 2.27 .

$$
\begin{aligned}
n_{e} & =\frac{n_{\|} n_{\perp}}{\sqrt{n_{\|}^{2} \cos ^{2} 0+n_{\perp}^{2} \sin ^{2} 0}} \\
n_{o} & =n_{\perp} \\
\Delta n & =n_{e}-n_{o}=0
\end{aligned}
$$

Consequently $I=0$, the birefringence vanishes for a sample aligned homeotropically. Irrespective of the sample orientation relative to the crossed polarizers, it appears dark due to complete extinction of the transmitted light. Thus, LC samples aligned homeotropically are also referred to as pseudo-isotropic [72]. On the other hand, for samples aligned unidirectionally, $\phi=\pi / 2$, and correspondingly $\Delta n=n_{e}-n_{o}=n_{\|}-n_{\perp}$. The transmitted intensity now varies only with $\varphi$ (see equation 2.30). As a result of this, a uniformly aligned sample appears dark (akin to homeotropic) when the optical axis is parallel (or perpendicular) to one of the polarizers. The maximum of the transmitted intensity is obtained at a sample orientation of $\pi / 4$ relative to the crossed polarizers. The schlieren textures presented in Fig. 2.11 evolves due to different orientations of the nematic director within a confinement possessing random anchoring conditions.

Essentially, rotation of the sample stage or the crossed polarizers forms the first step towards reconstruction of the in-plane director field within a nematic sample. Owing to the quadratic dependence of the transmitted intensity, $I$, on $\varphi: I \propto \sin ^{2} 2 \varphi$, the transmitted signal obtained for symmetrical values of $\varphi= \pm \varphi_{0}$ cannot be distinguished. For this purpose an optical retardation plate was used, inserted between the sample and analyzer, at angle of $\pi / 4$ relative to the crossed polarizers. The quartz retardation plate is characterized by two primary 

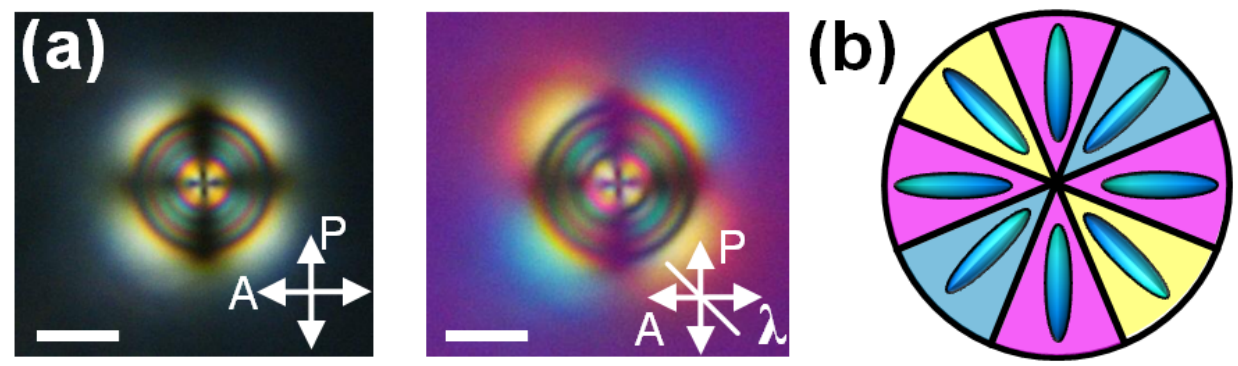

Figure 3.6: Optical retardation using $\lambda$ plate. (a) Polarized micrographs of a colloid with perpendicular anchoring on its surface, dispersed in a homeotropic matrix of nematic 5CB. Insertion of the $\lambda$ plate (on the right) helps to distinguish regions of similarly bright intensity (left micrograph). (b) Colour scheme showing LC director field observed with a $\lambda$ plate at $\pi / 4$ relative to the crossed polarizers.

axes: fast and slow along which the refractive indices are minimum and maximum respectively. In the present work, a retardation plate producing a phase shift of $530 \mathrm{~nm}$ was used (also known as $\lambda$ plate). The regions of minimum intensity appear red due to the reduced phase retardation. Correspondingly, the brighter regions acquire different colours depending upon the orientation of the director relative to the fast and slow axes. As an example, the director field around a colloid particle within a homeotropic matrix of 5CB is shown in Fig. 3.6. The bright regions observed between crossed-polarizers show different colours on insertion of the $\lambda$ plate. Figure 3.6b represents the colour scheme expected from different director orientations on introduction of a $\lambda$ plate.

\subsubsection{Fluorescence confocal polarization microscopy}

"...the study of liquid crystals is complicated because it involves several different scientific disciplines... and also a certain sense of vision in three-dimensional space in order to visualize complex molecular arrangements."

The above lines are taken from the preface written by P. G. de Gennes for the first edition of his book The Physics of Liquid Crystals [151]. The need for a three-dimensional visualization of the LC ordering can hardly be overemphasized [152]. Although different techniques available through polarization optical microscopy can be utilized to resolve the director orientation, the results are, at best, in-plane projections of the director field, obtained as an integrated image of the actual $3 D$ configuration. The technique of confocal microscopy, 
conceptualized by Marvin Minsky [153], however, heralded the beginning of a high-resolution point-by-point image construction method. Combined with fluorescence imaging, confocal fluorescence microscopy has emerged as an integral part of modern science [154-156].

Through the seminal work of Smalyukh, Shiyanovskii, and Lavrentovich [157], the knowledge of confocal fluorescence microscopy was extended to fluorescence confocal polarization microscopy (FCPM) technique, which resolves the director field within an LC sample spatially in the three dimensions. FCPM is based on the working principles of the conventional confocal technique, however with two fundamental modifications:

(i) use of a polarized light source for illuminating the sample, and

(ii) replacing usual fluorescent dyes with long dye molecules aligning along the $\mathrm{LC}$ director. A list of dye molecules suitable for the FCPM technique can be found in reference [158].

Figure 3.7 a schematically shows the FCPM working principle employed in this work. A polarized laser was used to excite the fluorescent dye molecules, oriented along the local director field. Alternatively, unpolarized light of a specific wavelength can be first passed through a polarizer before guiding it to the dye-doped LC sample [159]. The fluorescence signal from the dye molecules was directed to the detection unit using a dichroic mirror. Additionally, the fluorescent light is passed through a pinhole to reduce the amount of defocussed light entering the detector.

The selection of the appropriate fluorophore is based on the alignment of the dye molecules along the liquid crystal molecules: The transition dipole moment of the fluorescence dye is parallel to the $\mathrm{LC}$ director. When linearly polarized light excites the fluorescence, maximum emission signal obtained from those regions where the director and laser polarization are parallel. Conversely, the minimum intensity corresponds to mutually perpendicular orientation of the laser and LC director. In general, the fluorescence intensity $I_{F C P M} \propto \cos ^{4} \phi$, where $\phi$ refers to the laser polarization relative to the director. Additionally, due care needs to be taken regarding the dye concentration and intensity of the exciting laser. At high laser intensities the nematic molecules can reorient due to the strong optical field [160].

The three dimensional director field in the present research was resolved with the help of the fluorescent dye Nile Red (Sigma Aldrich, Germany). 5CB was doped with a small amount of the fluorescent dye $(0.01 \%$ conc. by weight). The molecular structure of Nile Red is shown in Fig. 3.7k. The dye molecules, which align along the long molecular axis of 5CB [161], thus trace the NLC director field when excited using light of appropriate wavelength. An optimal fluorescence signal $\left(\lambda_{e x c}=553 \mathrm{~nm}, \lambda_{e m}=640 \mathrm{~nm}\right)$ was obtained using linearly polarized laser light, $\lambda=543 \mathrm{~nm}$ ) at a power of $50 \mu \mathrm{W}$. The laser power was low enough to avoid any light- 


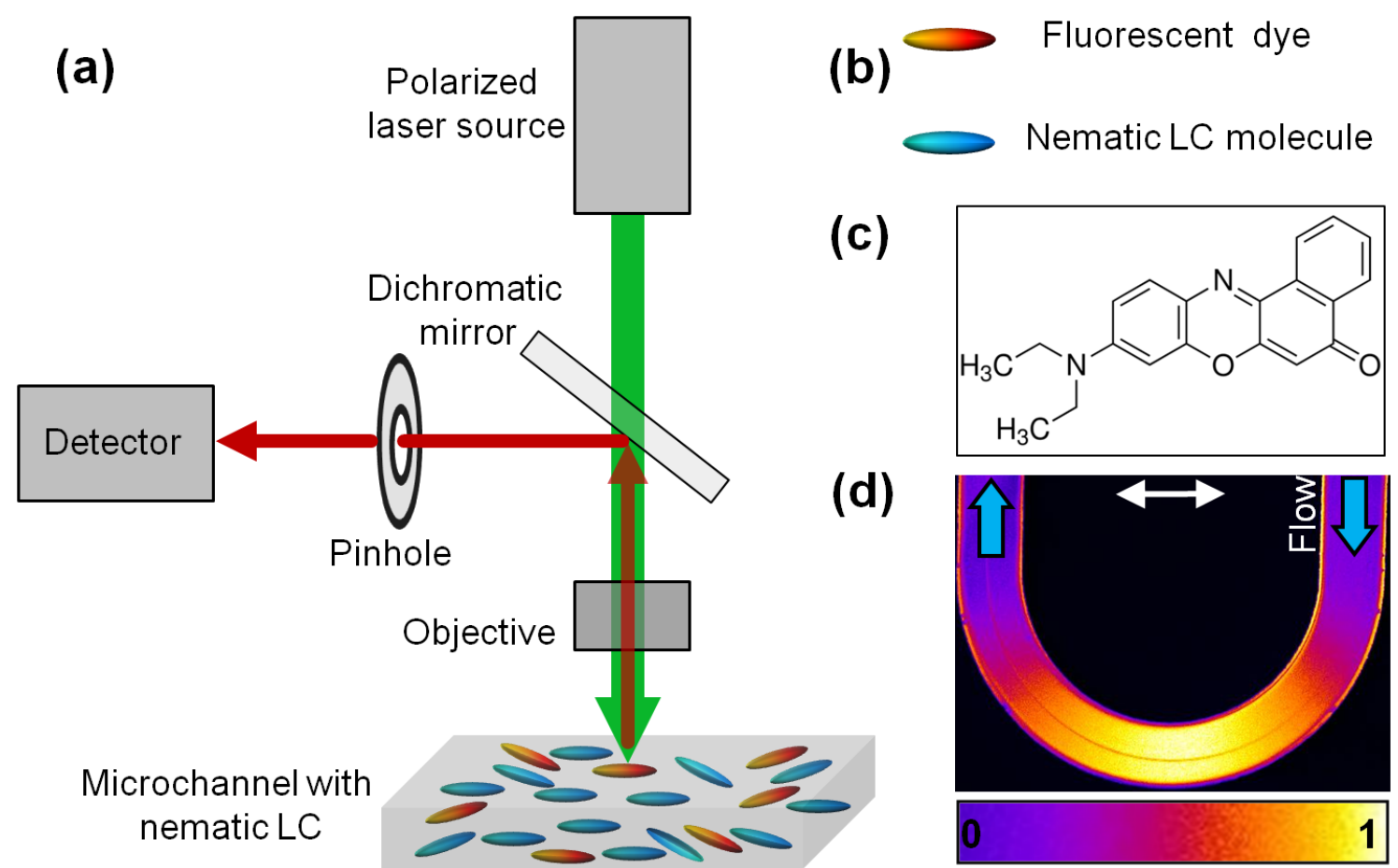

Figure 3.7: Fluorescence confocal polarization microscopy (FCPM). (a) Working principle of the FCPM technique [159]. (b) The nematic and dye molecules are shown in blue and red colours respectively. (c) Molecular structure of the Nile Red dye. (d) Example showing detection of the flow-aligned director field within a U-shaped microchannel ( $x y$ image at $z=$ $d / 2$ ). The channel width is $100 \mu \mathrm{m}$. Bright signal ( 1 on the intensity scale) is observed in the regions where the director is parallel to the laser polarization (double-headed white arrow). Minimum intensity ( 0 on the intensity scale) is obtained when the director and laser polarization are mutually perpendicular. For other intermediate angles, the signal intensity varies between 1 and 0 .

induced reorientation and photobleaching of the dye molecules. FCPM experiments were conducted in two different scan directions: $x y$ scanning with stacking in $z$ direction, generally at $500 \mathrm{~nm}$ intervals, and $y z$ scanning with stacking in $x$ direction, typically at $750 \mathrm{~nm}$ intervals. In Fig. 3.7 d, the flow-induced director alignment is demonstrated within a U-shaped channel. The $x y$ micrograph was obtained at half of the channel depth $(z=d / 2)$. Additionally, in the experiments, the direction of the laser beam polarization has been varied between from 0 to 180 degrees relative to the NLC sample. By combining the FCPM measurements at different scanning planes and the POM data, the three dimensional director profile was reconstructed. 


\subsubsection{Particle tracking method}

The majority of the velocity measurements has been carried out using standard particle tracking technique. The colloidal particles dispersed within the nematic host served as tracer elements in the measurements. Although colloidal particles self-assemble to form extended chains, such a possibility was avoided in the present system by keeping the colloid concentration very low, and by using the dispersions freshly after sonication. Using optical microscopy, movies of the nematic flow were acquired typically at a rate of $\approx 10$ frames per second. Depending upon the desirables from the experiments, this rate was altered between 1 and 25 frames per second. The image files were then analyzed using a standard algorithm of tracking and trajectory analysis [162] available through an open source image processing package, FIJI (formerly known as ImageJ). Due to the low tracer density and flow speeds typically less than $1000 \mu \mathrm{m} / \mathrm{s}$, the usual complications encountered in image analyses are generally absent in the current experiments.

\subsubsection{Dual-focus fluorescence correlation spectroscopy}

Dual-focus fluorescence correlation spectroscopy (2fFCS) complemented the particle tacking experiments. The technique was employed for measuring the velocity profile of the flowing LC, particularly in systems involving flow of an NLC matrix coexisting with topological defects. The use of particle tracking velocimetry for such coexisting systems is unsuitable due to the interaction of the tracer particles with the topological defects. For 2fFCS, the LC was doped with the lipophilic dye ATTO Oxa12 (Atto-Tec GmbH, Germany) at nanomolar concentration. The dye-doped LC was flowed within a microfluidic chip bonded to a microscopy cover slip. Measurements were performed on a standard inverted confocal microscope for time resolved fluorescence measurements (MicroTime 200 with dual-focus option, Pico-Quant $\mathrm{GmbH}$, Germany), schematically depicted in Fig. 3.8 [163]. Light from two identical pulsed diode lasers $\left(\lambda_{e m}=640 \mathrm{~nm}\right.$, pulse duration $50 \mathrm{ps}$ fwhm) with orthogonal polarization were combined with a polarizing beam splitter and coupled into a polarization-maintaining fiber. Both lasers were pulsed alternating with a repetition rate of $20 \mathrm{MHz}$, with an average laser power adjusted to $20 \mu \mathrm{W}$ each. The light from the fiber is collimated and reflected by a dichroic mirror into the microscope objective (UPLSAPO 60x W, 1.2 N.A., Olympus Deutschland $\mathrm{GmbH}$, Germany). The fluorescence signal is collected by the same objective and focused on a confocal pinhole (diameter $150 \mu \mathrm{m}$ ). The signal is further split by a polarizing beam splitter onto two single-photon avalanche diodes (SPCM-AQR-13, PerkinElmer Optoelectronics, 
Germany). Prior to each flow-profile acquisition, a cross-sectional image of the channel was acquired by moving the channel with the attached piezo electrical stage (P-562.3CD, Physik Instrumente, Germany). Flow profiles were acquired at discrete points along a line at half the height of the channel. Typical acquisition time was two minutes per point. Data analysis and fitting were subsequently performed using custom Matlab routines (Fig. 3.8p). 


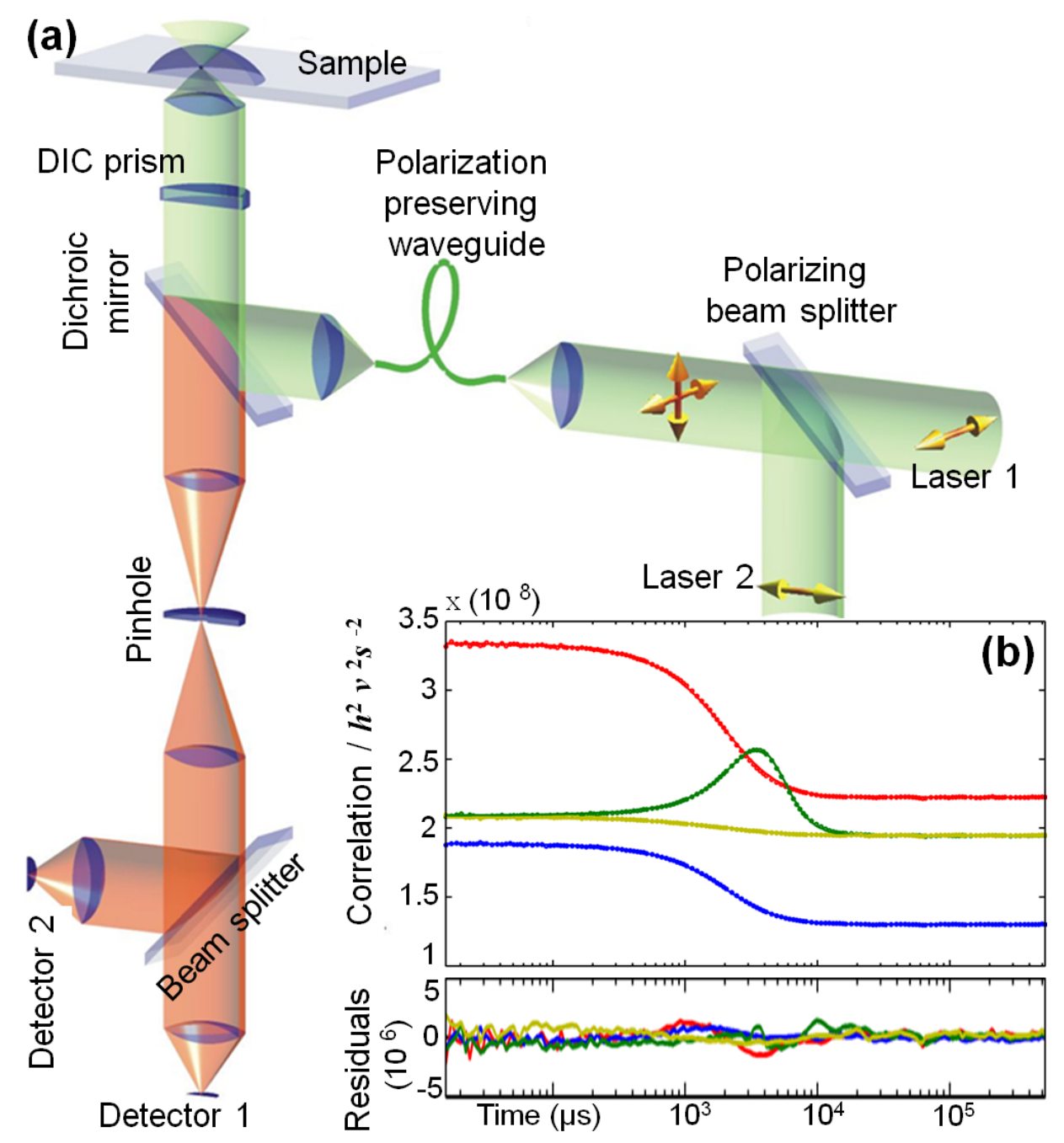

Figure 3.8: Flow velocity (v) measurement using 2fFCS. (a) Schematic of the 2fFCS setup. The light of two perpendicularly polarized laser beams is combined by a polarizing beam splitter, passed through an optical fiber, and reflected towards the objective by a dichroic mirror. Before entering the objective, the light is passed through a Normarski prism which deflects the light depending on its polarization. After focusing the beams through the objective, two overlapping foci are generated. The resulting fluorescence light is collected by the same objective and then focused through a confocal aperture and detected by two single photon avalanche diodes. (b) Typical 2fFCS correlation curves for Atto Oxa12 in NLC 5CB flowing through a rectangular microfluidic channel with a volume flow rate of $0.05 \mu \mathrm{l} / \mathrm{min}$. The four curves comprise two autocorrelations (one from each focus; blue and red curve) and the forward and reverse cross-correlations (green and yellow curve). Solid lines are fits to the data (squares). The data shown here correspond to measurements in the parallel orientation, and therefore show a maximum in the forward cross-correlation. Resulting flow rate as determined by 2fFCS measurement was $124.1 \mu \mathrm{m} / \mathrm{s}$. 


\section{Chapter 4}

\section{Functionalization of microfluidic devices}

Systematic studies of thermotropic liquid crystals in confinement, such as liquid crystals in microfluidic channels, require control of the anchoring conditions on the surfaces. Especially for the case of uniform planar anchoring, the standard method involves a mechanical treatment (rubbing) of the surface that is not applicable to microfluidic devices. In this chapter methods for the achievement of well-defined anchoring conditions in microfluidic channels consisting of polydimethylsiloxane and glass will be presented. The development of these techniques were motivated by the primary need to integrate the domains of microfluidics using soft lithography and investigations of LC flows. Various physico-chemical techniques developed during the course of this research enable to establish homeotropic, degenerate planar, uniform planar, and hybrid anchoring conditions on the surface of the channel walls. The director field for each case was analyzed using a combination of POM and FCPM techniques. Achievement of controlled surface anchoring of the LC molecules within microchannels is expected to be of growing importance, leading to novel possibilities in liquid crystal research in general.

\subsection{Non-trivial aspects of microchannel functionalization}

In the preceding chapters, we have acquainted ourselves with some unique attributes of liquid crystals as a material. We have (hopefully) appreciated the significance of surface anchoring and its influence on the equilibrium director field orientation. Microfluidic investigations, as in any other realm of LC research, thus require a precise knowledge and control of the anchoring conditions on the microchannel walls. For flat surfaces of macroscopic dimension, particularly motivated by the industrial application of display devices, extensive knowledge 
about the attainment of a desired anchoring condition exists [63, 66, 164]. However, these methods cannot, or only to a certain extent, be transferred to microfluidic confinements due to the small dimensions and possible complex architectures.

Liquid crystal anchoring conditions as the ones described in Chapter2(Fig.2.5), are generated on substrates by employing a variety of chemical and physical methods [63, 66, 164, 165]. In spite of their wide acceptance in both application and research of LCs, attempts to transfer the technology in microfluidic systems have never been reported. This is partly attributed to the inherent nature of the functionalization methods. Many of these techniques employ mechanical processes on open, macroscopic substrates, which cannot be extended to functionalize confinements having micrometer dimensions. For instance, the technologically important case of uniform planar anchoring is usually obtained by coating the surface with a thin polymer layer followed by a mechanical rubbing. In a microfluidic device, such a process is not feasible due to a number of reasons. The scale of the confinement inhibits the use of any intrusive method. After the fabrication of the microfluidic device, functionalization can be effected either by flow or some non-intrusive (e. g. optical) techniques. Consistent functionalization along the flow path adds to the challenge especially with increasing complexity of the microchannel architecture. The fabrication of the microfluidic devices requires the PDMS and glass surfaces to be exposed to plasma (which usually is the final step) for effecting strong bonding between the elements. This process modifies the surfaces chemically and thus negates the effects of any chemical treatment carried out beforehand. The methods presented here demonstrate ways to functionalize microchannels after the fabrication step. As an alternative approach, substrates possessing specific anchoring conditions can be bonded using parafilm [44] or spacers, to create a functionalized microfluidic environment. However, such channels are restricted only to simple geometry and the channel depth is dependent on the film/spacer thickness. The surface morphology and anchoring on the film/spacer lead to additional inhomogeneities. The use of patterned surfaces [166] and non-intrusive techniques [167], though promising, face practical constraints in being explored as generic routes to microfluidic functionalizations.

The methods discussed here are highly compatible with the channels fabricated using standard soft lithography techniques [146]. The general scheme of the coordinate system which will be followed hereupon is presented in Fig. 4.1. In the first segment of the chapter we shall look into modifications of the surfaces that are relevant from a microfluidics perspective and determine the wettability and surface anchoring of NLC molecules on them. The second part focuses on the realization of the surface anchoring (degenerate planar, uniform planar, 


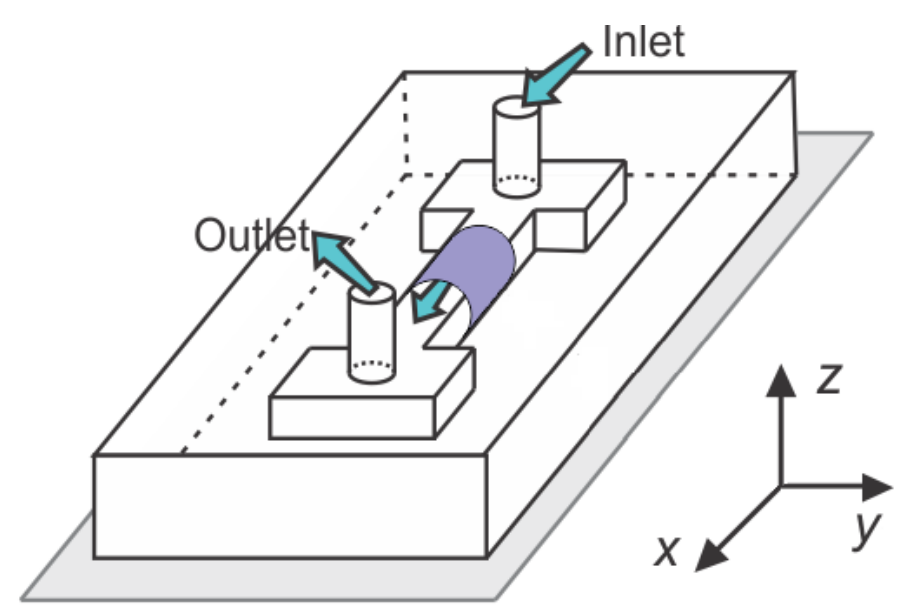

Figure 4.1: Cartesian coordinates defining the micro-flow. The flow direction is along $x$, the channel width along $y$, and the channel depth is along $z$.

homeotropic, and hybrid) within the microfluidic confinements. Although the functionalizations have been carried out within simple linear channels with a rectangular cross-section, the methods can be equally applied for architectures of higher complexity. The channel dimensions considered here are depth $d \approx 30 \mu \mathrm{m}$, width $w \approx 50 \mu \mathrm{m}$, and length $l=20 \mathrm{~mm}$. The orientation of the LC molecules on the channel walls was characterized by measuring the bulk molecular orientation in absence of any external field with the help of polarizing optical microscopy (POM) and fluorescence confocal polarizing microscopy (FCPM). Different regions along the channel length were observed under microscope to estimate the uniformity of functionalization. The section is concluded with comments on the homogeneity and temporal stability of the functionalization carried out within the microchannels.

\subsection{Anchoring characterization of functionalized substrates}

Microfluidic devices fabricated using soft lithography have two chemically distinct surfaces, namely PDMS and glass (channel bottom). In absence of any surface treatment, PDMS is hydrophobic whereas glass is hydrophilic in nature. PDMS and glass surfaces were characterized for wettability and anchoring with 5CB in standard laboratory conditions. The substrates were cleaned thoroughly with isopropanol and dried by blowing nitrogen gas. Possible traces of isopropanol were driven off by placing the surfaces in a thermal chamber $\left(110^{\circ} \mathrm{C}\right.$ for 30 min). The dynamic sessile drop method was used to estimate the wettability of the NLC on 


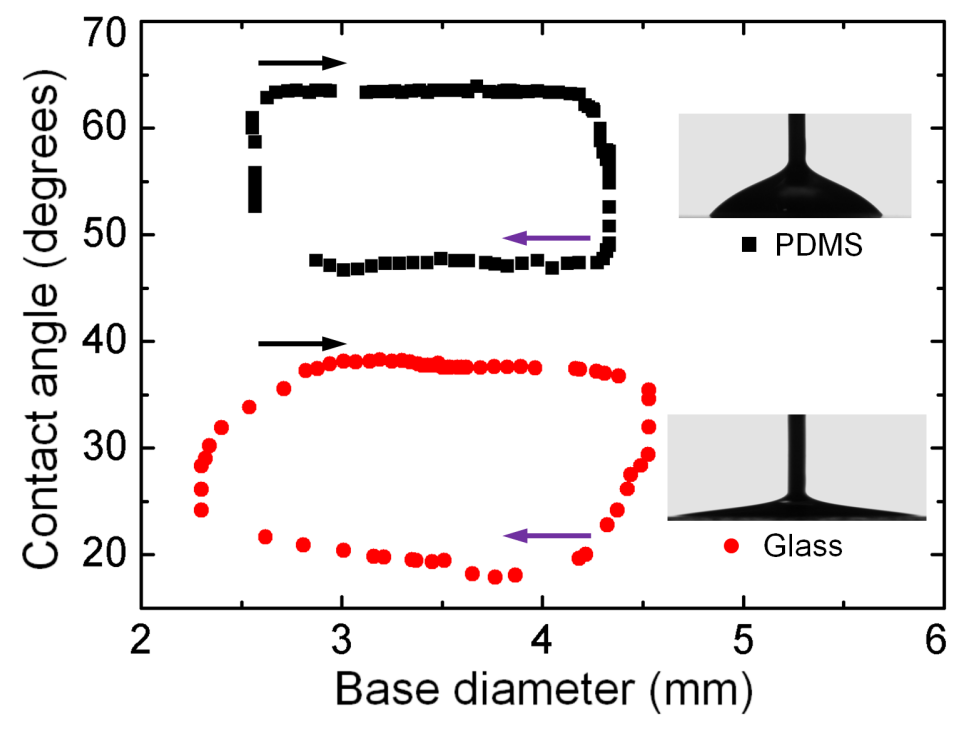

Figure 4.2: Wettability characterization of glass and PDMS surfaces. Using dynamic contact angle measurements (sessile drop method), wettability of 5CB in nematic phase on PDMS (black squares) and glass (red circles) is presented. The black and purple arrows indicate the directions of the advancing and the receding contact angles, respectively.

the surfaces. Measurements were carried out for advancing and receding contact angles $(C A)$ of 5CB on PDMS and glass using a contact angle goniometer (Dataphysics OCA), which was calibrated for deionized (DI) water on both PDMS and glass. Figure 4.2 shows the measured contact angle hysteresis curves for $5 \mathrm{CB}$ on PDMS and glass surfaces. The $C A$ of $5 \mathrm{CB}$ on PDMS was found to lie within $\approx 63^{\circ}$ degrees (advancing) and $\approx 47^{\circ}$ (receding), indicating lower wettability relative to glass $\left(C A\right.$ varied between $18^{\circ}$ and $\left.38^{\circ}\right)$.

The other important surface property in this study is the anchoring of $5 \mathrm{CB}$ molecules on the substrates, resolved using POM and FCPM measurements. A standard LC cell is a single dimension confinement (only depth), comprising two substrates separated by spacers of fixed height. LC cells were prepared using thin glass substrates or glass coated with PDMS, which were separated by a pair of spacer strips with adhesive on either side $(\approx 30 \mu \mathrm{m}$ high $)$. The cells were filled up with 5CB in the isotropic phase using capillary action and were allowed to cool down to nematic phase. From Fig. $4.3 \mathrm{a}$ and b, degenerate planar anchoring on glass substrates is concluded through the observed POM textures. Since there is no preferential alignment of the director $\vec{n}$ along any direction, the molecules orient randomly forming a multidomain texture of different orientations of $\vec{n}$. Existence of the topological defects (Section 2.6) is 

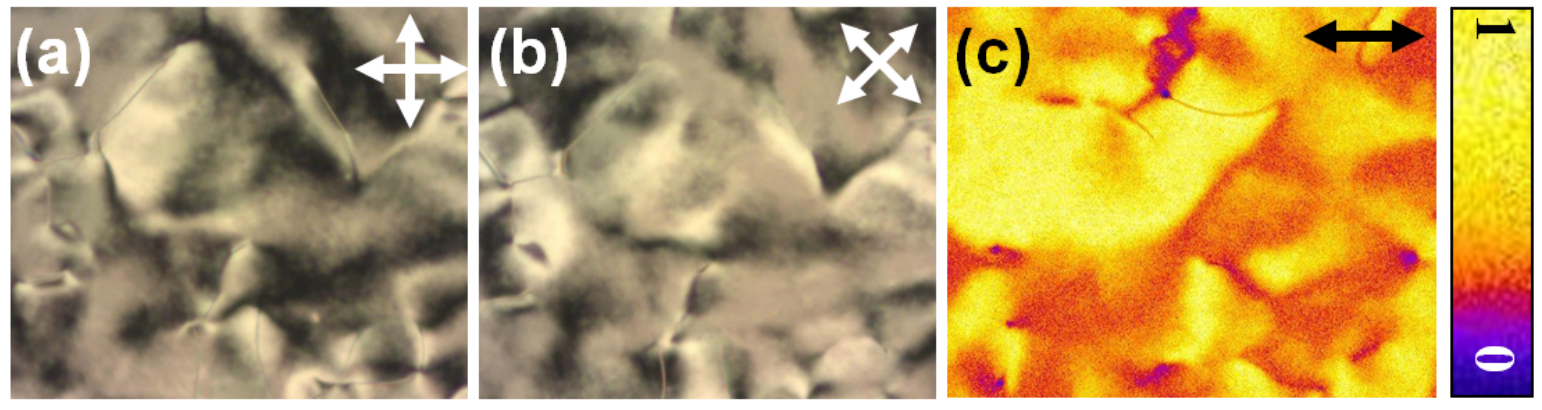

Figure 4.3: LC anchoring on untreated glass surface. (a), (b) Polarizing optical micrographs of $5 \mathrm{CB}$ confined in a glass LC cell observed between crossed polarizers at relative orientations of 0 and $\pi / 4$. (c) Corresponding FCPM micrograph obtained with polarized laser. The polarization state is denoted by the double-headed arrow. The domain structures observed through polarized light and fluorescence intensity distribution indicate random orientations of $\vec{n}$ in the azimuthal plane of the cell (degenerate planar anchoring). Colour intensity of 1 and 0 respectively correspond to molecular orientation parallel and perpendicular to the laser polarization in (c).

also a common feature of the NLCs confined between substrates possessing degenerate planar anchoring. Scattering of light by the defects and spatial variation of the transmitted light intensity through the sample indicate the presence of degenerate anchoring of the nematic $5 \mathrm{CB}$ on the surface. Additional FCPM measurements support the POM observations and confirm the presence of domains with random orientations of $\vec{n}$ within the LC cell. The fluorescence intensity distribution in the $x y$ plane is shown in Fig. 4.3.

Significantly low transmission of light was observed when 5CB was confined in PDMS cell and placed between crossed polarizers. Irrespective of the sample rotation on the azimuthal plane (Figure $4.4 \mathrm{a}$ and $\mathrm{b}$ ), the light transmission was found to be minimum. On introducing the Bertrand lens, concentric circular fringes with an extinction cross [150] were observed, as shown in Fig. 4.4k. The arms of the cross were parallel to the polarizers and remained static on rotation of the sample on the microscope stage. The conoscopic micrographs in addition to the FCPM studies confirm homeotropic orientation of the LC molecules in the bulk of the PDMS cell, in agreement with the available literature [168].

The aforementioned techniques were subsequently used for the characterization of the surfaces after they had been modified using various physico-chemical means. The surfaces were treated with each of the following agents: oxygen plasma, DMOAP, polyvinyl alcohol (PVA, average $\mathrm{Mw}=90 \mathrm{k}$ from Sigma Aldrich), and the well-known photoaligning polymer para- 

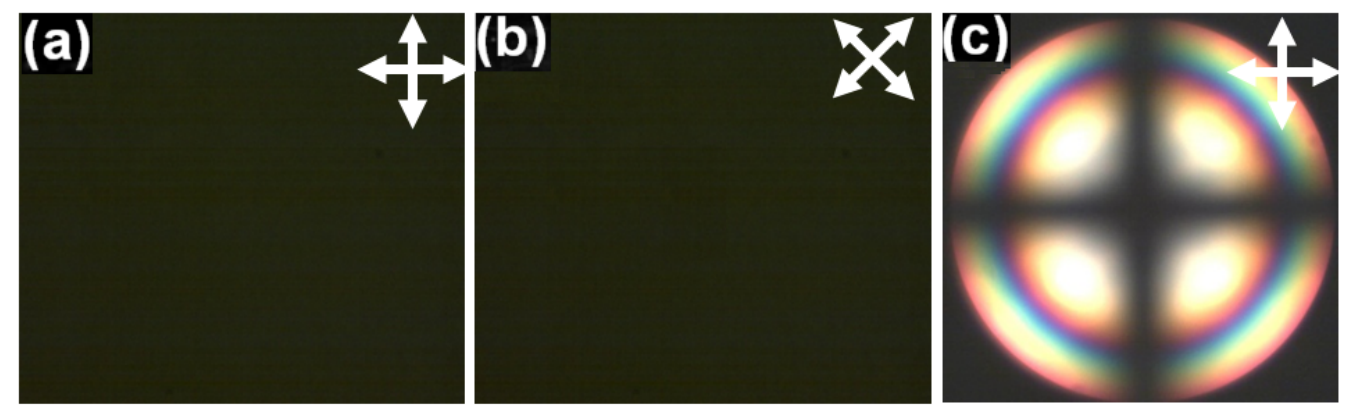

Figure 4.4: LC anchoring on untreated PDMS surface. (a), (b) Polarizing optical micrographs of 5CB in a PDMS LC cell for 0 and $\pi / 4$ orientations of the crossed polarizers. (c) Conoscopy of the sample shows the typical cross pattern, confirming homeotropic orientation of the molecules.

fluoro polyvinyl cinnamate (PVCN-F) [169]. Low molecular weight PVCN-F was employed as a solution (conc. $10 \mathrm{~g} / \mathrm{l}$ ) in 1,2 dichloroethane and chlorobenzene (1:1), whereas DMOAP and PVA were employed as aqueous solutions in deionized water (conc. $0.1 \%$ by weight). Further details will be provided at appropriate sections of this chapter.

Table 4.1 summarizes the wettability and anchoring of 5CB molecules that resulted from the surface modifications. Subsequent to exposure to oxygen plasma, both PDMS $\left(11-29^{\circ}\right)$ and glass $\left(11-20^{\circ}\right)$ show similar range of contact angles. The contact angles (lower value: receding and higher value: advancing $C A$ ) were measured within 10 minutes after plasma treatment. It can be concluded from the dynamic $C A$ measurements that both the glass and the PDMS surfaces possess similar orders of surface energy after exposure to plasma. Thus, exposure to plasma helps in reducing the otherwise inhomogeneity between the surfaces properties of the two materials. Correspondingly, the surface anchoring of NLCs on PDMS changes from homeotropic [39] to degenerate planar. Though PDMS becomes hydrophilic after plasma exposure, over time $(1 \mathrm{~h})$ the unreacted monomers from the PDMS bulk and the ambient humidity revert the surface back to a hydrophobic one [170, 171]. The hydrophilicity can be nevertheless retained by appropriate methods, to be discussed later in this chapter (Section 4.3.1). The plasma treated glass, on the other hand, undergoes a marginal increase in hydrophilicity and exhibits degenerate planar anchoring as well.

It is well known that an aqueous solution of DMOAP (Section 3.2) is an effective orientant of LCs on glass surfaces [63]. The alignment of the silane molecules influence the LC molecules to orient homeotropically. Current experiments suggest that DMOAP is an effec- 


\begin{tabular}{|c|c|c|c|c|}
\hline Substrate & Plasma $\mathrm{O}^{*}$ & DMOAP & PVA & PVCN-F \\
\hline \multirow{2}{*}{ Glass } & $11^{\circ}-20^{\circ}$ & $42^{\circ}-79^{\circ}$ & $8^{\circ}-22^{\circ}$ & $15^{\circ}-24^{\circ}$ \\
\hline & $\overline{\mathrm{DP}}$ & Homeotropic & $\mathrm{DP} \rightarrow \mathrm{UP}$ & $\mathrm{DP} \rightarrow \mathrm{UP}$ \\
\hline \multirow{2}{*}{ PDMS } & $11^{\circ}-29^{\circ}$ & $49^{\circ}-70^{\circ}$ & $13^{\circ}-28^{\circ}$ & $21^{\circ}-33^{\circ}$ \\
\hline & $\mathrm{DP}$ & Homeotropic & $\mathrm{DP} \rightarrow \mathrm{UP}$ & $\mathrm{DP} \rightarrow \mathrm{UP}$ \\
\hline
\end{tabular}

Table 4.1: Dynamic contact angle measurements for 5CB on functionalized glass and PDMS surfaces. The lower and higher values respectively indicate the receding and the advancing contact angles. Surface anchoring of 5CB on modified substrates were experimentally investigated and denoted here by DP (degenerate planar) and UP (uniform planar).

tive orientant also for PDMS (see Table 4.1). Treatment of glass with DMOAP significantly reduced its wettability as is evident from the $C A$ values $\left(42-79^{\circ}\right)$. In comparison, PDMS undergoes a marginal reduction $(\approx 10 \%)$ of wettability. The silanization of the substrates produced a homeotropic anchoring, which was observed to be stable for months.

PVA, when spin-coated and annealed on plasma treated glass and PDMS surfaces, gives degenerate planar orientation of the LC molecules. However, when rubbed unidirectionally, the LC molecules orient along the rubbing direction. The $C A$ s for PVA-coated glass lie between $8^{\circ}-22^{\circ}$ and that of PDMS between $13^{\circ}-28^{\circ}$. Compared to the existing PVA deposition method [172], the one presented here helps to bypass the intermediate silanization step.

We used a well-known photoalignment method [169] and the polymer PVCN-F to achieve a uniform planar anchoring of the LC on the substrates. Structurally, PVCN-F has a PVA main chain and photosensitive side-groups of fluorinated cinnamic acid. PVCN-F was spincoated on plasma-exposed surfaces $\left(2000 \mathrm{rpm}\right.$ for $30 \mathrm{~s}$ ) and baked at $150^{\circ} \mathrm{C}$ for $1 \mathrm{~h}$ after which linearly polarized ultraviolet (UV) light was normally incident on them. The polymer side-groups undergo cross linking, leading to a structural anisotropy that is subsequently transferred to the LCs, giving rise to a homogeneous orientation of LC molecules. The molecules generally align on the azimuthal plane in a direction perpendicular to the UV polarization. Alternative photosensitive polymers can effect the LC alignment even parallel to the UV polarization [165]. The contact angle values of PVCN-F coated surfaces are slightly higher than those with PVA coating on respective surfaces (Table 4.1). However, wettability of such films can be tuned either by temperature or by varying the irradiation time of the UV light [173]. 


\subsection{Microchannel functionalization and characterization of surface anchoring}

In spite of the disparate properties exhibited by PDMS and glass surfaces, similar attributes are realizable through appropriate physico-chemical processes. The desired surface properties were transferred to the fabricated microchannels through reliable engineering and minimal complexity. For characterization, the functionalized microfluidic devices were first filled with $5 \mathrm{CB}$ in the isotropic state and left to equilibrate at room temperature for $\approx 12 \mathrm{~h}$. Thereafter, the surface anchoring was inferred by resolving the three dimensional director field within the microchannels.

\subsubsection{Degenerate planar anchoring}

Since the fabrication of microfluidic devices involves plasma treatment of the surfaces in the final step (Section 3.3.1), the inherent anchoring of LC molecules in a freshly prepared microchannel is degenerate planar on all four walls. Once the channel has been filled with $5 \mathrm{CB}$, the molecular orientation in the bulk is observed to remain stable for days. However, the hydrophilicity of the PDMS degrades steadily unless stored under conducive conditions, e. g., by immersing it in deionized water or keeping it in a thermal chamber, both of which prolong the hydrophilicity of the surface [174].
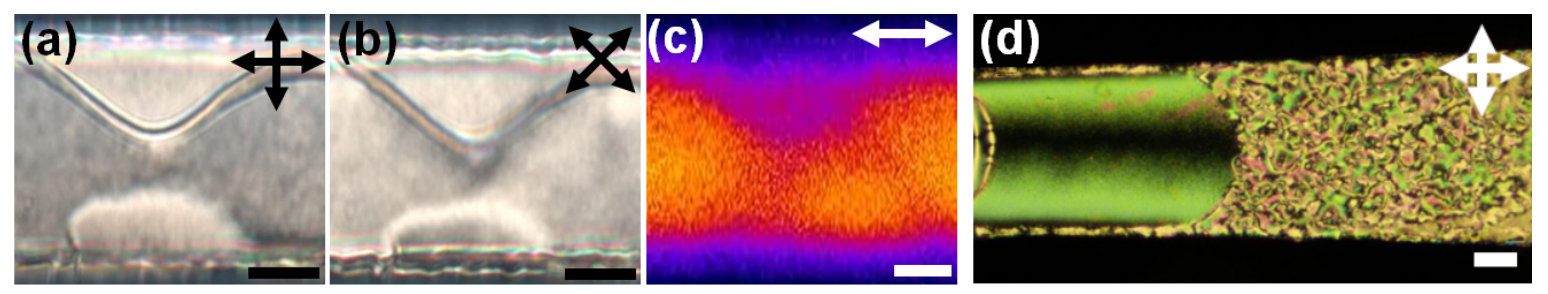

Figure 4.5: Degenerate planar surface anchoring. (a), (b) Polarizing optical micrographs of 5CB in microchannel functionalized for degenerate planar anchoring, observed between crossed polarizers at two different orientations indicated and the resulting (c) FCPM image of the channel azimuthal plane, obtained with a laser polarization along the channel length (indicated by the double-headed arrow). The fluorescence intensity distribution indicates domains with different orientations of $\vec{n}$ in the azimuthal plane of the cell. (d) Polarizing optical micrograph showing flow-induced alignment of in a degenerate channel (from left till middle of the channel). Scale bar in the micrographs corresponds to $15 \mu \mathrm{m}$. 
When observed between crossed polarizers, characteristic birefringent domains of randomly oriented 5CB appear as dark and bright regions as shown in Fig. $4.5 \mathrm{a}$ and b. The non-homogeneous intensity distribution in the FCPM micrograph (Fig. 4.5 ) of the functionalized microchannel confirms the realization of degenerate planar anchoring on the channel walls. The overall random orientation of $\vec{n}$ can be verified with the help of the normalized fluorescence intensity plotted as a function of the channel depth (Fig. 4.8). Strong fluctuations $\approx 70 \%$ of the average intensity indicate the presence of randomly oriented domains of LC molecules within the microchannel. If the channel is filled up in nematic phase, the occasional generation of a flow-induced easy axis is observed in some regions of the microchannel. The homogeneous birefringence in Fig. $4.5 \mathrm{~d}$ is representative of such a flow-induced alignment. It is interesting to note here that the alignment occurs in the narrower arm (higher flow velocity) of the diverging microchannel, indicating an apparent dependence on flow velocity.

\subsubsection{Uniform planar anchoring}

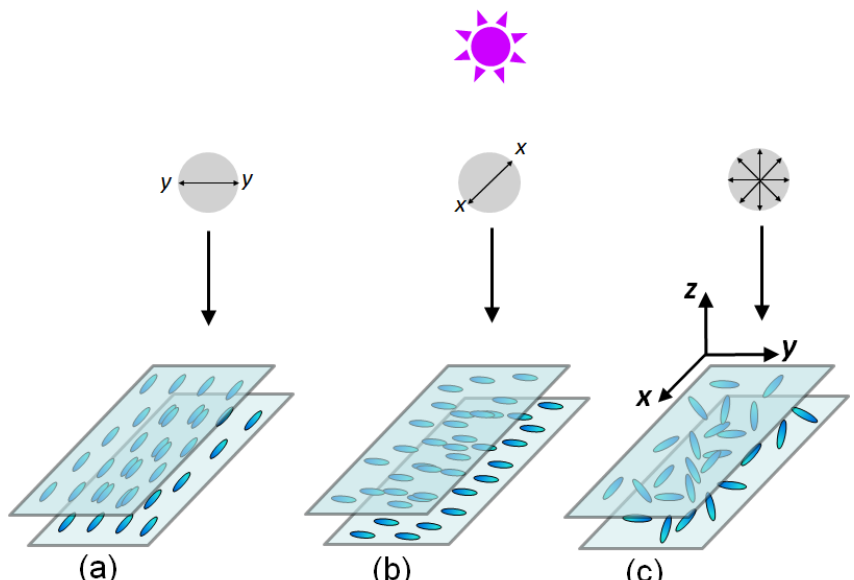

(a)

(b)

(c)

Figure 4.6: Photoalignment technique to generate uniform planar surface anchoring in microchannels. (a) When UV light (polarized perpendicular to flow direction) was incident on microchannel coated with PVCN-F, effective anchoring along the flow path was obtained. (b) Uniform planar surface anchoring along the channel width is achieved when the incident UV light was polarized along the flow direction. (c) Degenerate planar anchoring is obtained when unpolarized UV light was used.

A multi-step approach was followed for rendering uniform planar surface anchoring to the microchannels. PVCN-F was deposited on the channel walls by running the solution through freshly prepared microchannels for $\approx 2 \mathrm{~min}$. The thickness of the deposited PVCN-F 


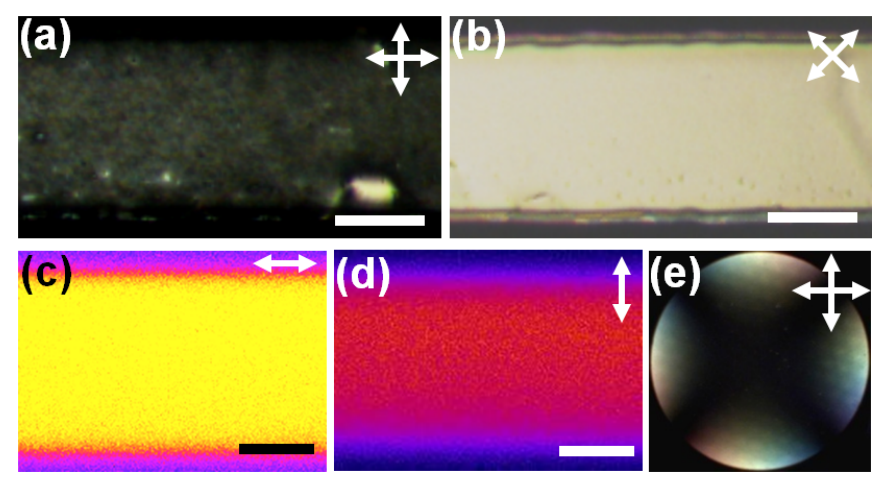

Figure 4.7: Uniform planar anchoring in microchannels using photoalignment method. (a) POM micrograph showing realization of LC anchoring along the channel. The sample was imaged between crossed polarizers, with one of the polarizers parallel to the resultant director orientation. (b) POM micrograph when the sample is rotated by $\varphi=\pi / 4$ shows a bright signal. (c) FCPM imaging of the director field in the $x y$ plane. The uniformly high fluorescence signal confirms the LC orientation along the laser polarization (indicated by the double-headed arrow). (d) The signal is reduced to minimum on changing the laser polarization by $\pi / 2$. (e) Characteristic conoscopy micrograph obtained from the functionalized channel. The scale bar corresponds to $20 \mu \mathrm{m}$

film was characterized using spectral interferometry techniques after it was stabilized via thermal treatment $\left(150^{\circ} \mathrm{C}\right.$ for $\left.1 \mathrm{~h}\right)$. The reflection spectrum, obtained by illuminating the coated layer with a white light source, was analyzed with the help of a spectrometer (AvaSpec-2048USB2, Avantes). Table 4.2 shows the thickness of the deposited PVCN-F on glass and PDMS. The layer thickness however could be altered by varying the time over which the solution was run through the channel. The microchannels were subsequently irradiated with polarized UV light ( $\approx 60$ min in Polylux PT UV cabinet). By varying the polarization of the UV light, alignment either parallel or perpendicular to the flow path was achieved, as is represented in Fig. 4.6 and b, respectively. In absence of any preferred direction of polarization of the UV light, degenerate planar anchoring is obtained on the channel walls (Fig. 4.6 c).

For uniform planar anchoring we expect a single birefringent domain possessing a homogeneous appearance throughout the sample. Figure $4.7 \mathrm{k}$ - e show optical micrographs of the equilibrium orientation of the $\mathrm{LC}$ molecules within a functionalized microchannel. The channel in this particular example was irradiated with UV light polarized along the channel width, thus generating alignment parallel to the length of the microchannel. The uniform planar orientation of the LC molecules was observed between crossed polarizers $(\varphi=0$ and $\varphi=\pi / 4)$ 


\begin{tabular}{|c|c|}
\hline Polymer - Substrate & Deposited thickness (nm) \\
\hline \hline PVCN - PDMS & $34 \pm 4$ \\
\hline PVCN - Glass & $96 \pm 10$ \\
\hline PVA - PDMS & $54 \pm 6$ \\
\hline PVA - Glass & $120 \pm 10$ \\
\hline
\end{tabular}

Table 4.2: Thickness of polymer deposition on different substrates calculated from reflection spectrum. PVA thickness on glass was obtained using AFM measurements.

as shown in Fig. $4.7 \mathrm{a}$ and b. A high fluorescent intensity was obtained with an excitation laser polarized along the channel length (Fig. 4.7k). This however reduced to minimum when the polarization direction was rotated by $\pi / 2$, shown in Fig. $4.7 \mathrm{~d}$. Furthermore, the characteristic hyperbolic isogyres, corresponding to uniform planar anchoring, were observed in conoscopy (Fig. 4.7k). The observations confirmed the uniform planar orientation of the LC molecules along the channel length.

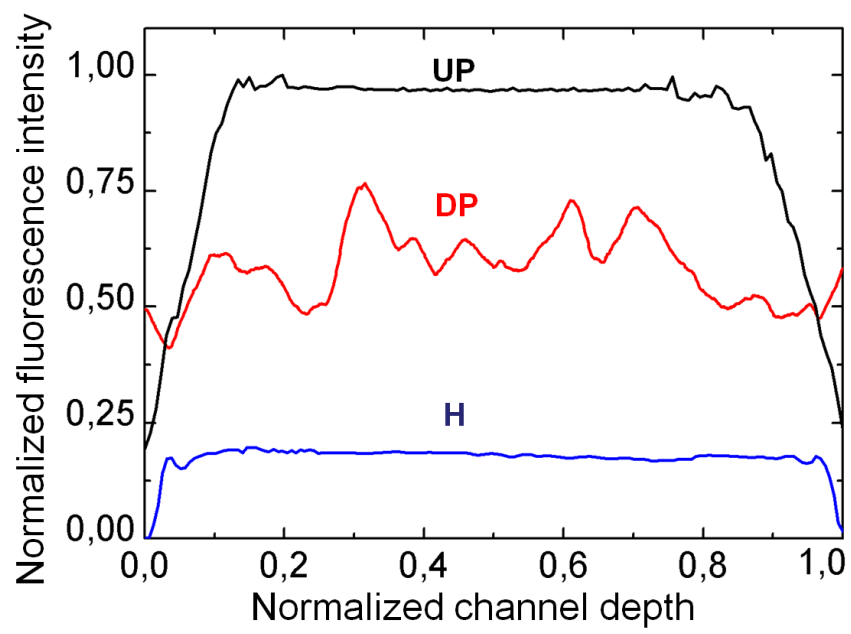

Figure 4.8: FCPM intensity distributions for functionalized microchannels. Normalized fluorescence intensity is plotted as a function of normalized channel depth. An overall high intensity uniformly distributed along the $z$ direction describes the homogeneity of the molecular orientation. Intensity distributions corresponding to degenerate planar (DP) and homeotropic (H) channels are represented comparatively.

The spatial orientation and uniformity of the alignment of the LC molecules within the functionalized channel were resolved by performing FCPM imaging along the cross-sections ( $y z$ plane). The results are presented as fluorescence intensity distributions along the channel 

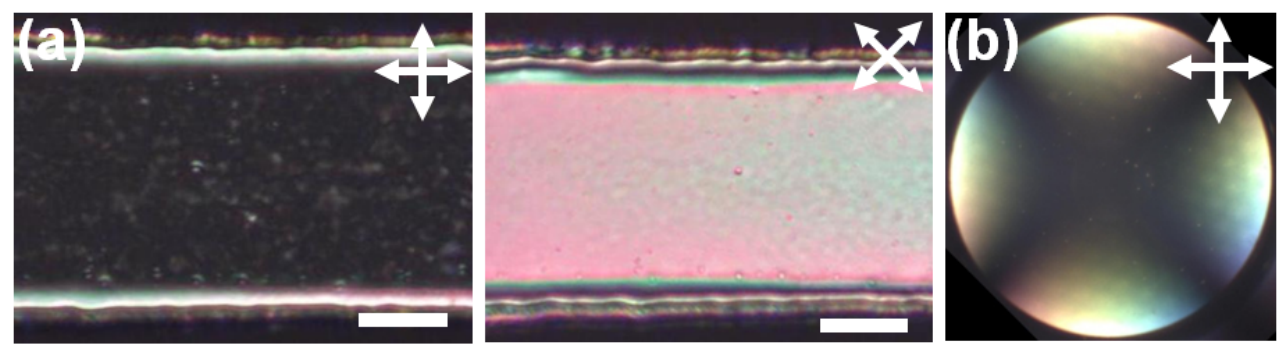

Figure 4.9: Uniform planar anchoring using pneumatic buffing. (a) POM images of microchannel functionalized by pneumatic buffing of PVA layer. (b) Conoscopic micrograph of the planar channel.

depth (Fig. 4.8). The variation of the intensity distribution was used as a measure of the spatial homogeneity of the equilibrium orientation within the functionalized microchannels. The distribution in the present case indicates a uniformly high intensity, except for regions very close to the walls, where local roughness leads to fluctuations of the signal. The distribution within the bulk shows low fluctuations, typically within $10 \%$ of the average fluorescence intensity. This is comparable with the range of intensity fluctuations obtained from a uniform planar LC cell. Combining POM and FCPM imaging, it is concluded that the LC anchoring is uniform planar within the microchannel. The anchoring has been found to be stable for the entire span of observation lasting for weeks.

Alternatively, a uniform planar anchoring could be achieved by a pneumatic buffing of a PVA coating on the channel surfaces. Freshly prepared microchannels were filled with an aqueous solution of PVA. The hydroxyl groups of PVA bond covalently to silanol groups on the glass surface [175]. Due to the presence of silanol groups on plasma treated PDMS as well, a similar mechanism attaches the PVA molecules to the PDMS surface. The bonding is additionally stabilized by baking the filled channel at $80^{\circ} \mathrm{C}$ for $15 \mathrm{~min}$ (soft baking) and followed by baking at $110^{\circ} \mathrm{C}$ for $1 \mathrm{~h}$ (hard baking). During the baking process, air was pumped into the channel at a low flow rate of $\approx 50 \mu \mathrm{l} / \mathrm{min}$ to assist the elimination of any residual moisture from the inner walls of the channel. On completion of the baking process, the random polymer chains could be aligned by continued infusion of air into the channel at high flow rate $(\approx 10 \mathrm{ml} / \mathrm{min}$ ) for $30 \mathrm{mins}$, producing an effect similar to mechanical buffing [176] of the polymeric chains. In contrast to the mechanical buffing, where shearing forces deform the polymer film, the viscous forces of air in pneumatic buffing aligned the PVA polymer chains in the direction of the air flow. The channel was thereafter filled with $5 \mathrm{CB}$ in the isotropic phase and left to equilibrate. Figure 4.9 presents the resulting anchoring within the microchannel. 
This method however encounters a number of limitations. Since the anchoring is induced due to the infusion of air, the resulting NLC orientation is necessarily constrained only along the channel length. Besides, the spatial homogeneity of the resulting alignment was relatively lower. Moreover, the pneumatic buffing produced a rather weak planar anchoring: Subsequent flow of 5CB along a direction opposite to that of the buffing frequently disturbed/reversed the anchoring induced by air-flow. Due to an especially low viscosity of air, the buffing action is envisaged to be rather mild.

\subsubsection{Homeotropic anchoring}

For homeotropic anchoring, the channel was first filled with a DMOAP solution for $\approx 10$ min. Thereafter, the channel was rinsed with deionized water and heated at $80^{\circ} \mathrm{C}$ for $15 \mathrm{~min}$ and at $110^{\circ} \mathrm{C}$ for $1 \mathrm{~h}$. After the functionalization, the channel was filled with $5 \mathrm{CB}$ in the isotropic phase and allowed to cool down to the nematic phase before characterizations were carried out.

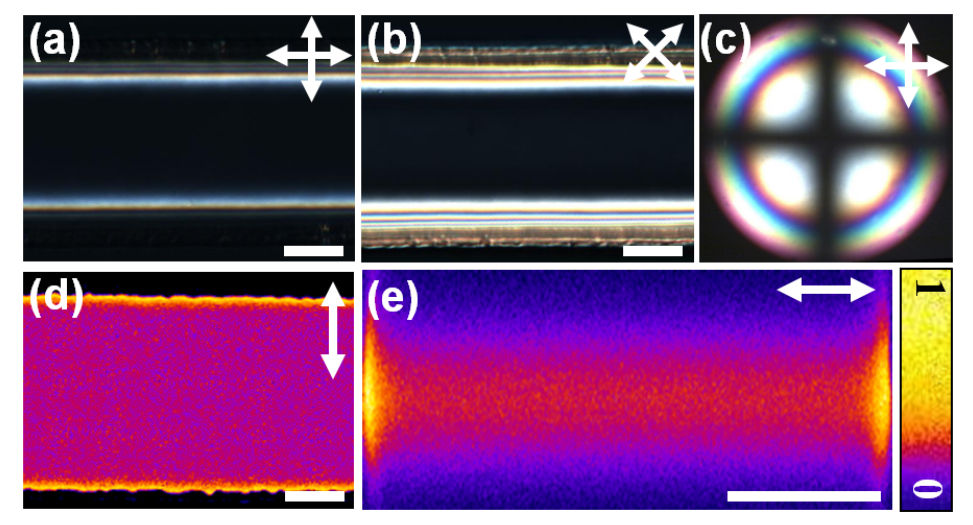

Figure 4.10: Homeotropic anchoring in microchannels. (a) Functionalized microchannel characterized between crossed polarizers. $\vec{n}$ is orthogonal to the azimuthal plane leading to minimum transmittance of light when the sample is placed between crossed polarizers. (b) No change of transmitted intensity is observed on rotation of the sample except close to the channel walls. (c) Maltese cross pattern observed when the sample is imaged conoscopically. (d) FCPM imaging in $x y$ plane yields minimum intensity, independent of the laser polarization direction. (e) The cross-sectional imaging shows uniform distribution of the intensity (minimum) across the channel depth. High intensity at the channel walls indicate that the LC molecules are parallel to the laser polarization (double-headed arrow). Scale bar corresponds to $15 \mu \mathrm{m}$.

Figure 4.10 summarizes the surface anchoring produced by the treatment of the chan- 
nel by DMOAP. When the sample was observed between crossed polarizers, the intensity of transmitted light was always low, independent of the polarizer position. However, close to the side walls of the channel, a higher transmittance is observed. Due to the homeotropic anchoring of the molecules, the director close to the side walls is confined on the azimuthal plane (Fig. 4.10 a, b). Consequently, an interference between the ordinary and extraordinary rays, with a maximum contrast at $\varphi=\pi / 4$, is produced in the POM micrographs. The order of the interference, indicated by dark and bright parallel lines near the walls, increased with channel depth. When the sample was viewed conoscopically, distinctive concentric circular fringes with an extinction cross [150] were observed (Fig. 4.10 c), representing homeotropic anchoring within the channel. The bulk orientation of $\vec{n}$ is further investigated using FCPM measurements. Figure $4.10 \mathrm{~d}$ and e respectively show the FCPM micrographs of the mid-plane $(x y)$ and the cross-sectional $(y z)$ view of the LC confined in the microchannel. In contrast to the uniform planar anchoring, the fluorescence intensity obtained from the $x y$ plane is close to minimum and is independent of the laser polarization. The distribution is found to be uniform $(\approx 0$ on the intensity bar) along the $z$ direction indicating that $\vec{n}$ remains homogeneously orthogonal to the confining surfaces. The perpendicular orientation of the LC molecules on the side walls was clearly captured when the laser polarization is parallel to the channel width, as shown in Fig. 4.10e. The intensity distribution across the channel depth (Fig. 4.8) lies around $15 \%$ of the average intensity for the uniform planar case. The microscopy results of the bulk orientation conclusively suggest the successful realization of a homeotropic anchoring on the channel walls. The anchoring was found to be inherently stable and is highly robust to temperature and humidity conditions.

\subsubsection{Hybrid anchoring}

The methods discussed in Section 4.3.1- 4.3.3 render identical surface anchoring properties on the surfaces that constitute the channel walls. However, the aforesaid techniques fall short of generating a continuous variation of the LC director (hybrid orientation) within the microchannel. In order to induce a hybrid orientation, different anchoring properties are desired on the confining surfaces. Consequently, different modification techniques are necessary for the same channel. To achieve this, the glass substrate and the PDMS mould were surface treated separately, nevertheless complying with the requirements of soft lithography.

The plasma treated glass surface was coated with PVA as described in Section 4.2. Due to overlapping refractive indices of glass and PVA, interferometry measurements for the film 

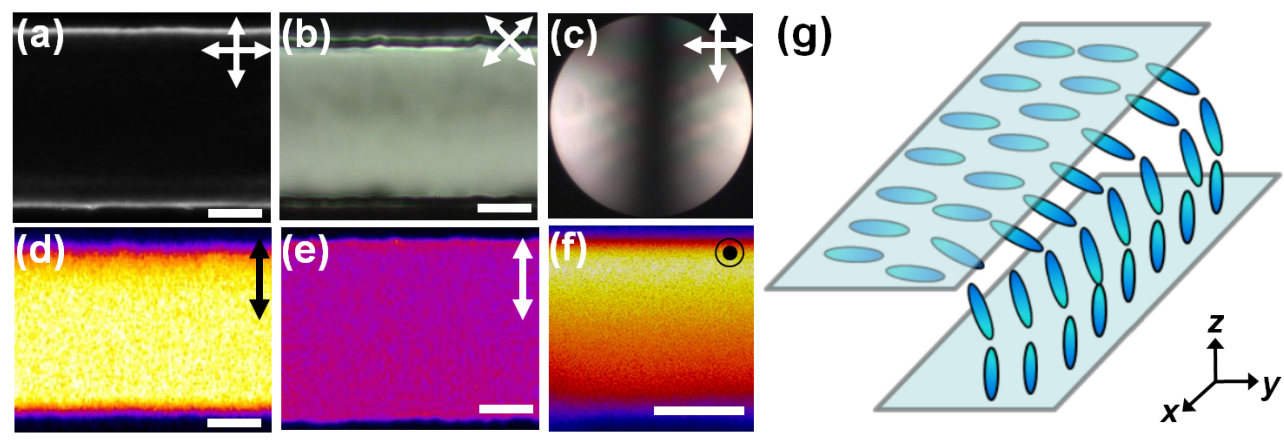

Figure 4.11: Microchannels functionalized for hybrid orientation. (a) Optical micrograph of a functionalized microchannel filled with $5 \mathrm{CB}$ shows very low transmission of light when placed between crossed polarizers, similar to homeotropic anchoring. (b) On rotating the sample by $\pi / 4$, the transmission reaches its maximum, similar to uniform planar anchoring. (c) Conoscopic image of showing the linear isogyre parallel to the direction of planar orientation. (d) High fluorescence signal is obtained close to the planar surface, and (e) low signal near the homeotropic surface. The laser polarization direction was fixed parallel to the planar alignment. (f) Cross-sectional view ( $z x)$ with the laser polarization perpendicular to the image plane. Gradual decay of the intensity distribution across the depth is observed. (g) Schematic representation of the resulting director pattern. Scale bar corresponds to $15 \mu \mathrm{m}$.

thickness are not suitable. Using atomic force microscopy (AFM), the thickness of the PVA layer was measured to be around $120 \mathrm{~nm}$ (Table 4.2). The polymer-coated glass substrate was then rubbed unidirectionally to generate uniform planar anchoring along the rubbing direction. Directly after exposing to oxygen plasma, the PDMS relief was mechanically pressed to the rubbed glass. This helps to bind the PDMS relief onto the PVA coated glass, through a mechanism similar to that of surface bonding of normal glass and PDMS in standard microfluidic device fabrication. Such a microfluidic device possesses degenerate planar anchoring on the PDMS surfaces and uniform planar anchoring on glass. When it is left for $\approx 12 \mathrm{~h}$ in ambient conditions, the PDMS recovers its homeotropic surface properties (owing to recovery of hydrophobicity) completely, whereas the glass surface still retains the uniform anchoring properties. This process effectively induces homeotropic anchoring on the PDMS walls and uniform planar anchoring on the glass wall.

In such confinements, the polar angle $\theta$ switches gradually from 0 (homeotropic) on the PDMS surface to $\pi / 2$ (planar) on the glass surface. Depending upon the placement of the PDMS relief on glass, the relative orientation of the flow-direction and direction of planarity can be tuned from 0 to $\pi / 2$. Thus, an entire range of relative angles between flow and director field can be obtained for studying flow in hybrid channels. For illustration, I have considered 
here a case where the direction of planar alignment is perpendicular to the flow sense. Between crossed-polarizers (one of the polarizers parallel to the channel width), one observes a minimum transmittance in $\operatorname{POM}(\varphi=0)$, as shown in Fig. 4.11a. As the sample was rotated by $\pi / 4$ (Fig. $4.11 \mathrm{p}$ ), the transmitted intensity monotonically increased. Though this is qualitatively similar to POM of uniform planar anchoring, the two cases were distinguished using conoscopy. In contrast to the hyperbolic isogyres of planar anchoring, the hybrid anchoring yields a linear isogyre along the direction of the planar alignment, as shown in Figure 4.11. In course of a rotation of the sample by $\pi / 2$, the linear isogyre first vanished at a position of $\pi / 4$, and reappeared at the perpendicular position. The spatial configuration of the director field is readily resolved by performing the FCPM measurements. Figure 4.11d and e respectively show the FCPM micrographs of the hybrid microchannel close to the two constituting surfaces. A bright intensity is captured close to the planar glass surface, (laser polarization parallel to the direction of planar alignment), whereas minimum intensity is obtained close to the homeotropic surface. The overall configuration was conclusively obtained from the crosssectional micrograph ( $z x$ plane) in Fig. 4.11f, demonstrating the continuous decrease of the fluorescence intensity from large values near the planar surface over intermediate values in the bulk to low values near the homeotropic surface, represented in Fig. 4.12. On combining the POM and FCPM measurements, the resulting three dimensional director field is shown schematically in Fig. 4.11g. The director field in the microchannel is observed to be stable for months.

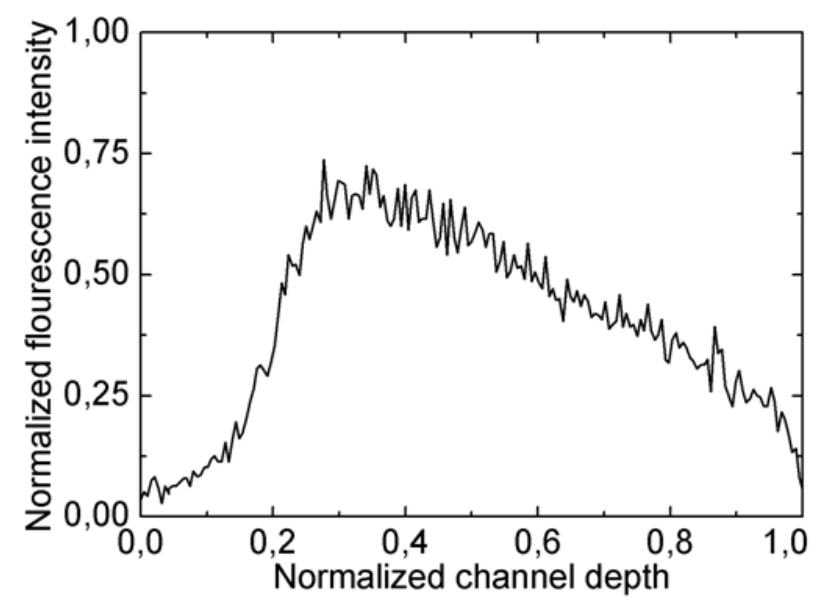

Figure 4.12: Fluorescence intensity distribution within a hybrid aligned microchannel. The intensity decays from maximum close to the planar surface to minimum near the homeotropic surface. 


\section{Chapter 5}

\section{Nematic liquid crystals confined within a microfluidic device: Static case}

Nematic liquid crystals within a microfluidic device offer interesting insights into the director equilibrium induced by the confining surfaces. Particularly, within microchannels possessing well defined boundary conditions, the nature of anchoring and proximity of the surfaces allow us to control the topology and evolution of defect structures. Furthermore, the surface-induced static equilibrium serves as the initial conditions for the subsequent flow phenomena. In this chapter we shall look into the static equilibrium of the nematic director field, and demonstrate experimental routes to controlled evolution of defects. By tuning the surface anchoring and channel dimensions, different topological possibilities are explored here. The relevant numerical simulations were carried out in collaboration with Miha Ravnik and Julia M Yeomans.

\subsection{Liquid crystals in confinements}

In Chapter 2, we have discussed that the director $(\vec{n})$ alignment in absence of any external torque is determined by the anisotropic interaction between the LC and the aligning surface. One of the more straightforward cases is that of an NLC droplet resting on a solid substrate. The LC droplet thereby experiences anchoring at two separate interfaces: LC-solid interface and LC-air interface. Given that the nematic anchoring at LC-air interface is typically homeotropic [177-179], the overall director field within the droplet is essentially determined by the boundary conditions at the solid substrate. For instance, if the solid substrate possesses uniform planar anchoring, then the director field is expected to equilibrate to a hybrid-aligned 
configuration (Section 4.3.4). Similarly, on replacing the LC-air interface by a solid substrate, different states of confinement-induced anchoring can be attained, as shown in Chapter 4 . Such confinements, popularly known was LC cells, are frequently employed both in research and in applications. In fact, by an appropriate selection of the surface anchoring, topological defects can be created with sufficient control and reproducibility [134, 180]. On the contrary, industrial applications rely on defect-free nematic samples. For instance, the display units present in personal computers, smart phones, or home-entertainment systems are various forms of LC cells possessing uniform alignment. A single characteristic length, the cell depth, is generally sufficient to describe the static or dynamic phenomena occurring within such confinements.

The presence of multiple boundaries, as in a microfluidic channel, enhances the complexity of the director interactions. In combination with appropriate surface properties, multiple boundaries provide the key towards incorporation of suitable topological constraints within the channels. Apart from the channel depth $d$, the channel width $w$ additionally contributes to the overall equilibration of the director. Nevertheless, the combined effects of the channel dimensions can be addressed by a non-dimensional parameter, aspect ratio, $A R=w / d$. Consequently, the limiting case $w \gg d, A R \rightarrow \infty$, defines the situation of an LC cell. Most of the experiments for the present work were done within microchannels with finite aspect ratio: typically spanning $1 \leq A R \leq 50$. In the following sections we shall try to appreciate the roles played by surface anchoring and channel aspect ratio in determining the static equilibrium of the nematic director within the microfluidic devices. The observations were carried out by filling the channel with 5CB in the isotropic phase and allowing it to cool down to the nematic phase at room temperature.

\subsection{Microchannels with planar surface anchoring}

Planar anchoring within microchannels signifies orientation of the LC molecules parallel to the confining surfaces. While degenerate planar anchoring typically furnishes the schlieren texture (Fig. 2.11), uniform planar orientation within the microchannel is expected to align the NLC molecules unidirectionally, without any defects (Fig. 4.7). And indeed this is the case: A gradual equilibration to the room temperature generally yields well aligned defectfree samples. However, in some cases of the microchannels with weak surface anchoring, as was observed on functionalizing the channel using PVA cum pneumatic buffing, domains of locally uniform planar director fields were observed. This is indicative of the weak anchoring 

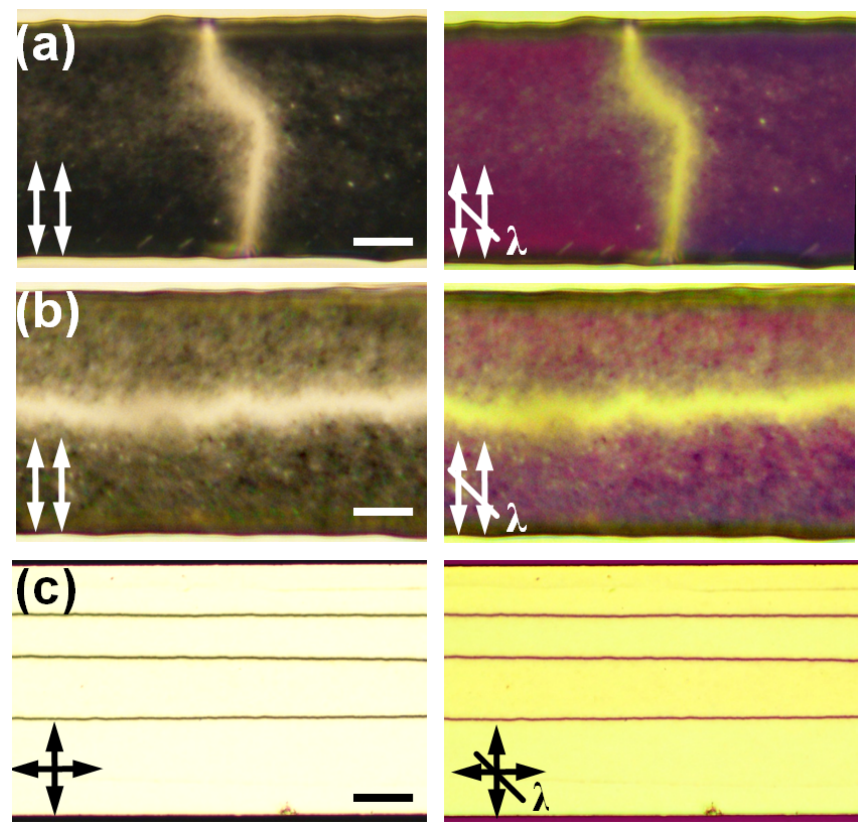

Figure 5.1: Static director equilibrium in channels having uniform planar anchoring. (a) Polarized micrographs with parallel polarizers show planar domains, separated by a domain boundary along the channel width, and (b) along the channel length. (c) Twist domains within uniform planar microchannel. Domains of opposite twists are separated by walls running along the channel length. The scale bar corresponds to $20 \mu \mathrm{m}$.

strength induced by the pneumatic buffing method. Some examples of such planar domains are presented in Fig. 5.1. The domain textures form spontaneously during the isotropic to nematic phase transition, and are separated by different kinds of wall structures. Consequently, the formation of such structures is beyond experimental control.

Figure 5.1 presents polarized micrographs of typical textures observed when the isotropic $5 \mathrm{CB}$ is cooled down to the nematic phase within planar microchannels functionalized using pneumatic buffing. In Fig. 5.1 a and b, the director field is confined to the azimuthal plane, and the domain wall characterizes a gradual in-plane bend deformation of the director. Such wall structures will be discussed in detail in the following chapter (Chapter 6.3). In Fig. 5.1k, the domain walls appear narrower than the ones discussed previously. Typically, they are formed due to the opposite director twists across the channel depth, and are referred to as twist walls [6]. The twists are distinguished from the image contrast using a $\lambda$ retardation plate. It is worthwhile to mention here that once formed, the twist domains are rather stable due to the relatively low values of the twist elastic constant, resulting in slow director relaxation. The implications of twist domains on the overall director field is far from trivial. The free energy 
minimization of the twist walls proceeds via complicated steps, generally setting off a cascade of defect dynamics: transformation of the wall to line defects (pincement) [181], and the interactions between the bulk and the surface disclination lines [68, 182, 183]. The formation of the twist or planarly aligned domains occurs at the phase transition of 5CB from isotropic to nematic phase. Consequently, the domain formation is a stochastic process that cannot be strictly controlled within a channel filled with 5CB. However, the appearance of domains could be suppressed either by filling up the channel in nematic phase, or by maintaining a flow during the phase transition. The viscous torque in this case biases the planar alignment necessarily along the flow direction. Once attained, the alignment was observed to be quite stable during the course of the experiments. It is conceivable that the side walls of the channels shall influence the overall director field as the aspect ratio is lowered.

\subsection{Microchannels with homeotropic surface anchoring}

Confinements possessing homeotropic anchoring, over the decades, have provided a very rich platform to study different aspects of topological defects. The investigations hitherto cover a wide range of homeotropic confinements: LC cells [178, 184-186], capillaries [99, 187-189], droplets [36, 190], micro-cavities [125, 191], and porous networks [192, 193]. In spite of the practical relevance of confinements with rectangular cross-sections, especially microchannels, the resulting equilibrium of the nematic director has not been experimentally analyzed yet.

The equilibrium nematic director in absence of flow was studied for a range of microchannel dimensions by varying the channel depth. Figure 5.2 presents polarization micrographs characterizing homeotropic anchoring on the walls of a $50 \mu \mathrm{m}$ wide and $\approx 15 \mu \mathrm{m}$ deep microchannel (Fig. 5.2). Close to the confining surfaces, the director field experiences a gradual elastic deformation to accommodate the existing boundary conditions. Effectively, bending of the director field near the surfaces alters the relative orientation of the optical axis and the light polarization, and generates ordinary and extraordinary components, which interfere to produce the dark and bright interference pattern parallel to the walls. Farther away from the confining surfaces, the LC director field equilibrated without any elastic deformation. Extinction of the transmitted light observed in the polarized optical micrographs (Fig. 5.2p and c) is supported by the in-plane FCPM image (Fig. 5.2 d). On scanning the sample at different horizontal planes $(x y)$ through the channel depth, the intensity of the fluorescence signal was found 

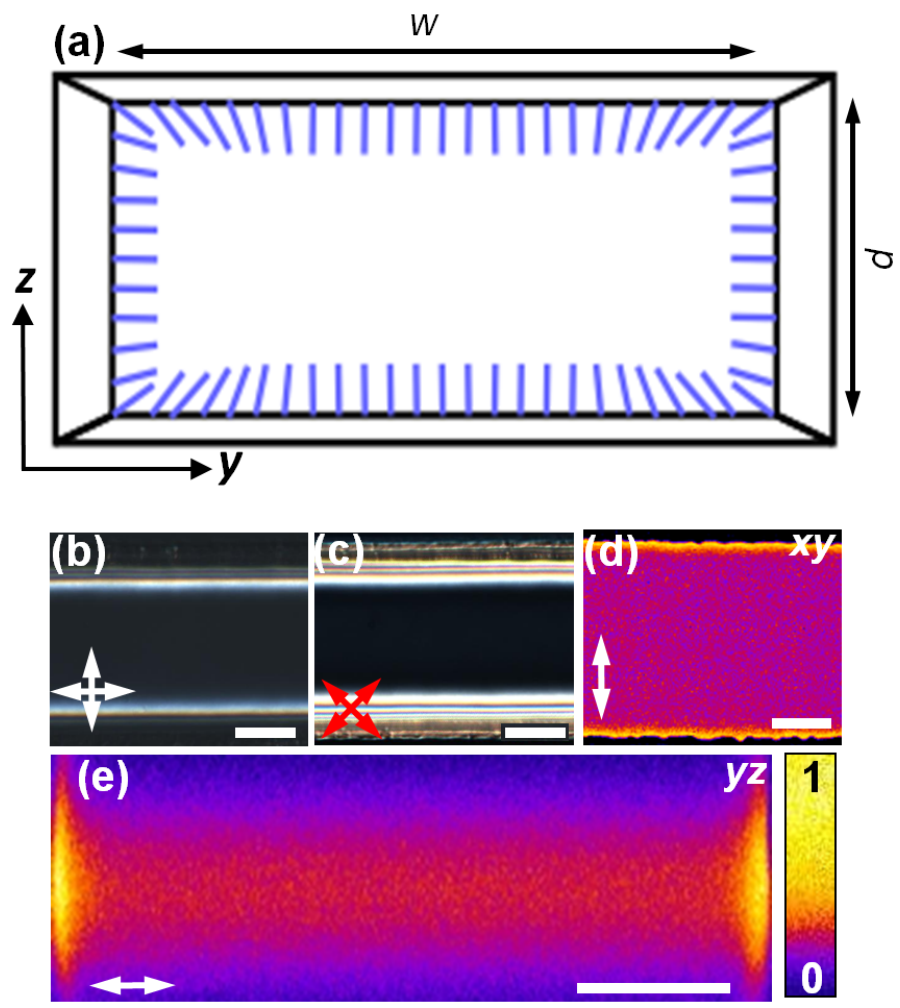

Figure 5.2: Nematic 5CB confined within a homeotropic microchannel. (a) Schematic representation of homeotropic anchoring on the channel walls. (b) and (c) show polarized micrographs of the channel at different orientations of the channel relative to the crossed polarizers. (d) In-plane FCPM image at $z=d / 2$. (e) FCPM image of the channel cross-section. The channel was $50 \mu \mathrm{m}$ wide and $\approx 15 \mu \mathrm{m}$ deep. The scale bar corresponds to $15 \mu \mathrm{m}$.

to be minimum irrespective of the direction of the laser polarization. The director field within the channel was indeed confirmed to be homeotropic, as seen in the cross-sectional view of the channel (Fig. 5.2e). Close to the side walls of the channel, high fluorescence intensity was noted since the nematic molecules oriented parallel to the laser polarization direction. In the rest of the sample, the intensity was uniformly low everywhere.

In comparison to the aforementioned channel, the optical micrographs corresponding to a shallower channel, $d \approx 5 \mu \mathrm{m}$, of similar width, exhibit visible differences (Fig. 5.3 $-\mathrm{d}$ ). The number of interference fringes appearing near the walls is reduced [76]. Consequently, the lateral spread of the interference pattern at the walls varied with the channel depth. As is shown in Fig. 5.3p, no significant interference is observed in a shallow channel. Due to the enhanced interaction between the horizontal surfaces (top and bottom) in a shallow channel, the elastic deformation close to the channel walls is inhibited. This is confirmed by the 
74 Chapter 5. Nematic liquid crystals confined within a microfluidic device: Static case

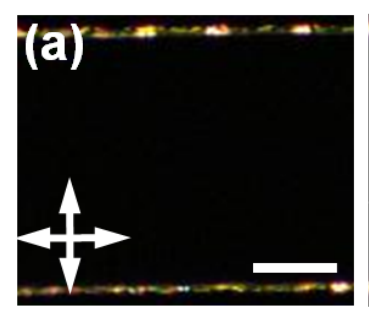

(d)
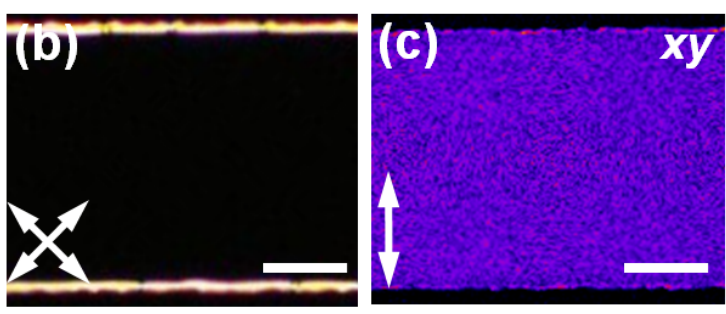

yz

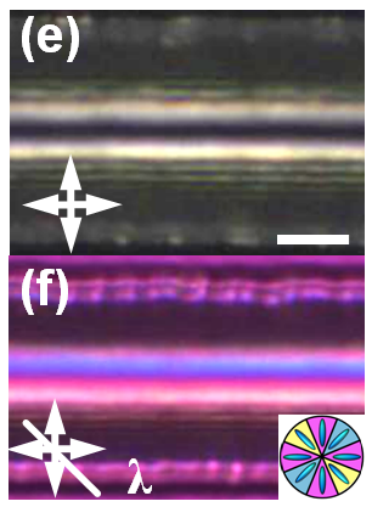

Figure 5.3: Influence of aspect ratio on nematic director equilibrium. (a) and (b) Polarized micrographs of a shallow channel at different orientations relative to the crossed polarizers. (c) and (d) Weak FCPM signal obtained from the in-plane $x y$ and cross-section $y z$ planes. (e) POM image of director escape in a homeotropic microchannel with $A R \approx 1$. (f) The director distortion in an escaped configuration identified by a $\lambda$ retardation plate. Scale bar: $5 \mu \mathrm{m}$ (a d), and $15 \mu \mathrm{m}(\mathrm{e}, \mathrm{f})$.

FCPM micrographs (Fig. 5.3 $\mathrm{c}$ and d). In addition to the overall fluorescence intensity from the bulk being weaker, the high fluorescence intensity near the walls observed previously, is suppressed as the director is strongly stabilized in the vertical direction. On the contrary, as the channel depth was increased, such that $A R \rightarrow 1(d \approx 50 \mu \mathrm{m})$, the anchoring contribution from the side walls matched up to that from the top and bottom surfaces. Thus, within a channel with $A R=w / d \approx 1$, the molecules have equal tendency to orient orthogonal to either of the $x y$ or $z x$ planes. Effectively this results in an integer singularity at the center of the channel, which however is seldom observed experimentally. Instead, the director conforms to an energetically favourable configuration by escaping in the third dimension (Fig. 5.3 and f), as is also observed in capillaries [188]. Such confinement-induced stabilization affects the director perturbation by flow: While even small fluctuations can reorient the director in deep channels, one requires stronger flows to induce the director deformation in shallow channels (Chapter6).

Subsequent numerical modeling provided further insights into the possible free energy states of nematic $5 \mathrm{CB}$ within homeotropic microchannels. The fluorescence confocal signal intensity $I$ was calculated from the local director $\vec{n}$ and laser polarization $\vec{P}$ as $I \propto(\vec{n} \cdot \vec{P})^{4}$ and POM micrographs were calculated with the Jones $2 \times 2$ matrix formalism [194]. Figure 5.4 summarizes the different possible static equilibria, and their corresponding FCPM and POM representations. For a given aspect ratio of the channel (calculated here for $A R=4$ ), four 
(a)
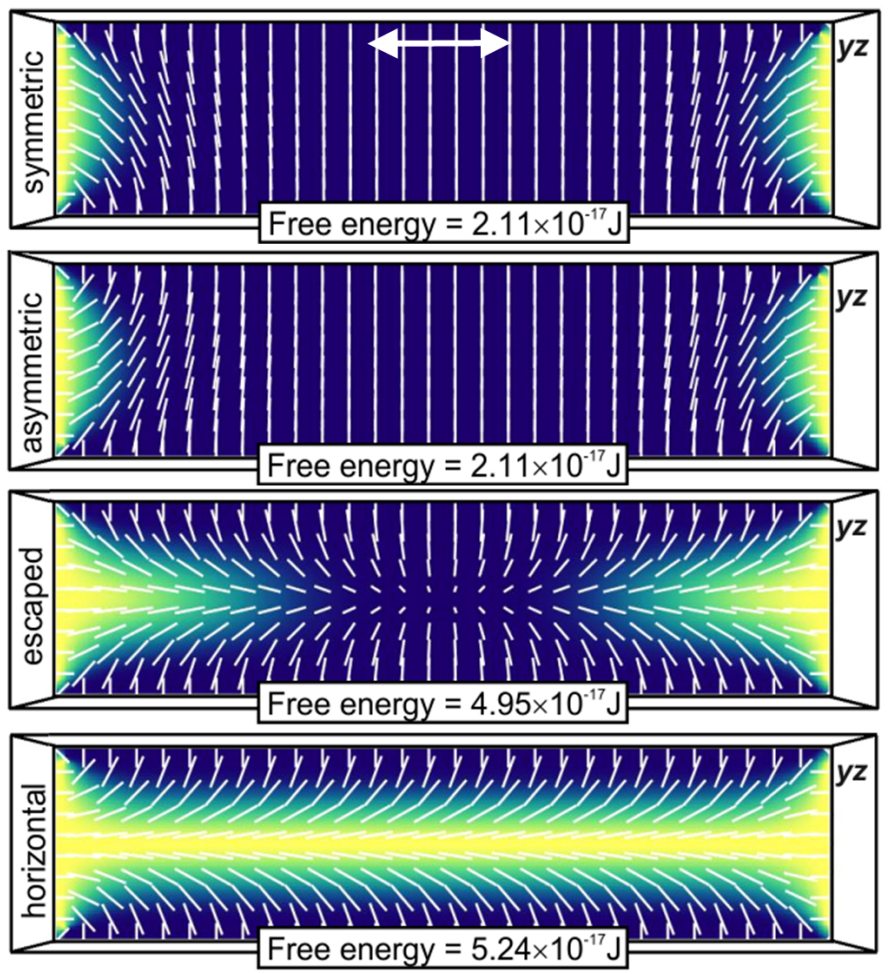

(b)
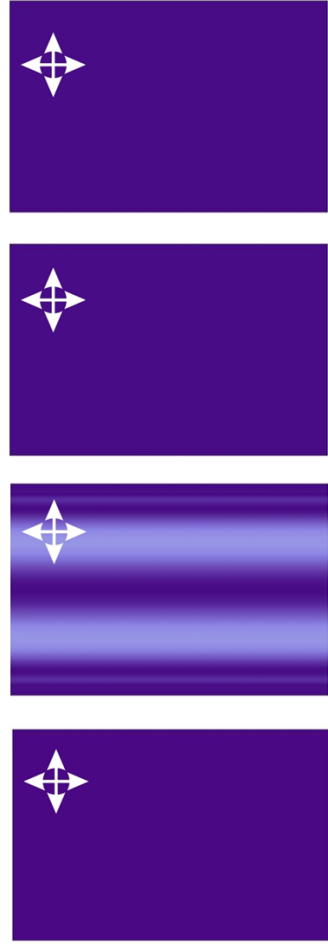

(c)
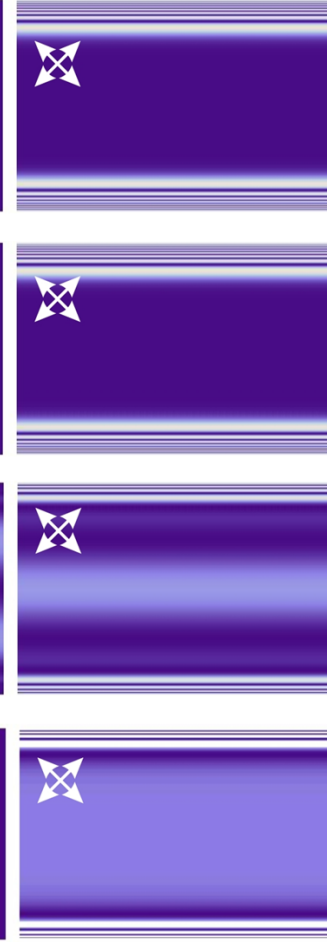

Figure 5.4: Numerical imaging of the static director field. (a) FCPM imaging of the channel cross-section reveals four different free energy states: symmetric, asymmetric, escaped, and horizontal. (b) and (c) Corresponding numerical polarized optical micrographs. Numerical modeling was carried out by Miha Ravnik.

different equilibrium director configurations were observed numerically: symmetric, asymmetric, escaped, and horizontal. While the symmetric and the asymmetric configurations corresponded to the lowest free energy states $\left(2.11 \times 10^{-17} \mathrm{~J}\right)$, the horizontal configuration was energetically least favourable $\left(5.24 \times 10^{-17} \mathrm{~J}\right)$. The free energy of the escaped structure was intermediate at a value of $4.95 \times 10^{-17} \mathrm{~J}$. Consequently, the occurence of the symmetric (or asymmetric) configuration is energetically favourable. This is in agreement with the experimental observations. However, both the symmetric and asymmetric orientations are optically identical, when observed numerically between crossed polarizers (Fig. 5.4p and c). As a result of this, these two cases are experimentally not distinguishable. Additionally, numerical modeling of channels having different aspect ratios shall help to verify other experimental observations reported here, especially the cases of shallow channels and channels with $A R \rightarrow 1$.

Interestingly, the numerical calculations do not suggest the creation and/or stabilization 
76 Chapter 5. Nematic liquid crystals confined within a microfluidic device: Static case

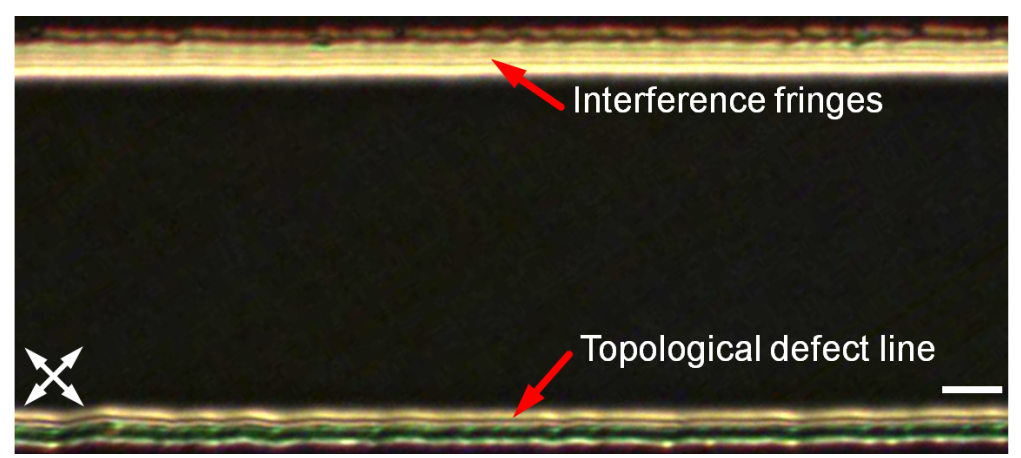

Figure 5.5: POM image shows a topological defect line parallel to the channel wall. Interestingly, close to the opposite wall, the expected interference pattern is observed. Due to scattering of light at the defect core, the line appears as thin dark line. The corresponding director field is presented in Fig. 5.6d. Scale bar: $15 \mu \mathrm{m}$.

of topological defects close to the channel walls. Instead, a continuous deformation of the nematic director field is observed in all cases. Although this is in reasonable agreement with the experimental findings, topological defect lines, extending along the channel, were also observed close to the side walls. One such instance is presented in Fig. 5.5, where the topological line and the expected interference pattern coexist in the same channel. The defect line appears as a thin dark line, due to scattering of light at the defect core. Possible reasons for this could lie in surface irregularities, and local curvature at the glass-PDMS bonding site. In the following section we shall discuss the evolution of topological defects close to the channel surfaces.

In addition to the nature of anchoring, the topological outcome of the director field is strongly influenced by the local curvature of the anchoring substrate. It is reasonable to expect that the microchannels possess curved (slightly rounded) corners, rather than perfectly perpendicular ones. Figure 5.6 shows different possible director deformations at the channel corners. While Fig. 5.6 shows continuous director deformation at all the channel corners, Fig. 5.6b - $\mathrm{d}$, present cases where the director is discontinuous at the topological defects, owing to the existing boundary and curvature conditions. Particularly, at perpendicular corners where the PDMS mould is surface-bonded to the glass substrate (shown as hatched lines), defect lines can be generated. Topologically the defect lines are semi-integer in nature. Figure 5.6d presents the director configuration relevant to the experimental observation of Fig. 5.5, in which both continuous and discontinuous director configurations exist at each of the PDMSglass corners. The experimental observations can be thus commented upon with the help of the surface curvature existing at the channel corners. 


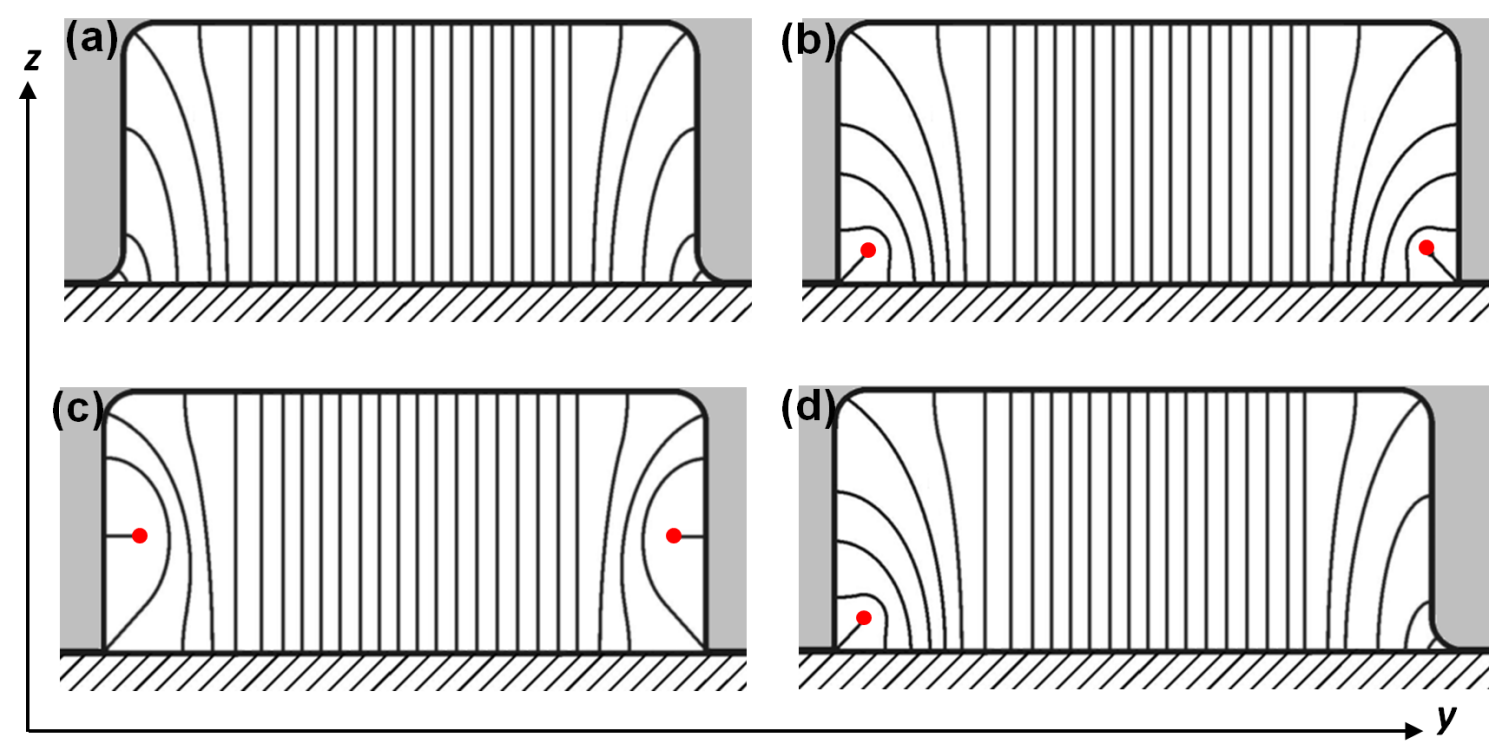

Figure 5.6: Director configuration at channel corners. (a) Absence of sharp corners leads to continuous director orientation. (b) - (d) Discontinuity in the director field results in evolution of semi-integer topological defects (red) at sharp corners formed by PDMS (grey hue) and glass (hatched lines) surfaces. The defect lines are parallel to the channel walls, going into the image plane. Schematic courtesy: Stephan Herminghaus.

\subsection{Homeotropic microchannel with cylindrical micro-pillar}

The soft lithography technique allows us to integrate micro-structures of different geometries and dimensions within the microchannel, e. g. micro-pillars of circular, and rectangular cross-sections. A schematic representation of such a microfluidic chip is shown in Fig. 5.7a. In this section we shall look into simple linear channels with a rectangular cross-section and a cylindrical pillar placed at the middle of the channel. The depth of the channel, $d$, was varied between $8 \mu \mathrm{m}$ and $50 \mu \mathrm{m}$, and the pillar diameter $2 r$ was chosen between $10 \mu \mathrm{m}$ and $60 \mu \mathrm{m}$ (Fig. 5.7p). All our experiments were conducted within $w \approx 100 \mu \mathrm{m}$ wide and sufficiently long (20 mm) channels. In Fig. 5.7 a, $x, y$, and $z$ denote the flow direction, channel width and channel depth, respectively.

The equilibrium director configuration of nematic $5 \mathrm{CB}$ was studied for a range of aspect ratios $(w / d)$ and pillar dimensions $(r)$. Figure 5.8 shows micrographs of a $(r, d, w)=(20$, $40,100) \mu \mathrm{m}$ channel filled with nematic 5CB, and observed under white light and confocal microscopes. The white light micrograph (top) clearly shows the channel boundary and the 
78 Chapter 5. Nematic liquid crystals confined within a microfluidic device: Static case
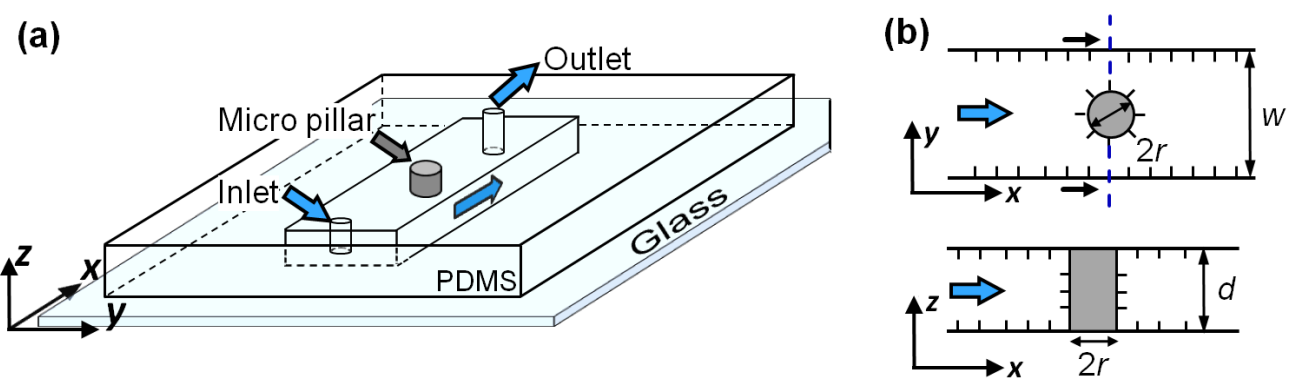

Figure 5.7: Microfluidic confinement with cylindrical micro-pillar. (a) Microchannel with a micron-sized cylindrical pillar positioned symmetrically in the flow path. (b) Schematic representation of the plan view and the cross-sectional view ( $x z$ plane position shown in the plan view). The channel and pillar surfaces possess homeotropic surface anchoring. $r, w$, and $d$ denote the pillar radius, channel width and depth, respectively.

cylindrical micro-pillar. When crossed polarizers were introduced, distinct dark and bright patterns were observed in the vicinity of the confining walls and the pillar surface. This is in accordance to the interference phenomenon discussed previously. Farther away from the pillar and the confining surfaces, the LC director field equilibrated without any elastic deformation. The whole-field director configuration was further confirmed from the FCPM measurements ( $x y$ scan, laser polarized along $y$ ). As seen in the FCPM micrograph (Fig. 5.87), a strong fluorescence signal is observed at the PDMS boundaries, whereas a weak signal is obtained from the rest of the channel. The lateral spread of the interference pattern at the surfaces varied with the channel depth. As is shown in Fig. 5.8 $\mathrm{p}$ (top), no significant interference is observed within a channel of depth $d \approx 10 \mu \mathrm{m}$. The reduction in the number of the interference fringes within shallow channels additionally led to a sharp appearance of the channel and pillar boundaries. On the contrary, the interference pattern is significantly prominent in the $(20,40$, 50) $\mu \mathrm{m}$ channel (Fig. 5.8p, bottom). By varying the aspect ratio $(w / d>1)$ of the channel, one can essentially tune the homeotropic coverage within the microchannels.

Figure 5.8c shows POM and FCPM images obtained in a $(30,40,100) \mu \mathrm{m}$ channel. The FCPM intensity distribution from the channel cross-section along sections 1 and 2 (Fig. 5.8 d) clearly resolves the homeotropic orientation on the involved surfaces. Interestingly, the lateral spread of the bend deformation at the side walls, seen as the bright fluorescence signal, is wider in section $\mathbf{1}$ as compared to that in section $\mathbf{2}$. This difference occurs due to larger elastic deformations effected by the micro-pillar. For a given aspect ratio, the larger the pillar size, the more significant is the elastic interaction. For example, a $40 \mu \mathrm{m}$ diameter pillar (Fig. 5.8 $\mathrm{a}$ ) reduced the lateral spread by $\approx 50 \%$, whereas, the presence of a $60 \mu \mathrm{m}$ pillar reduced the 

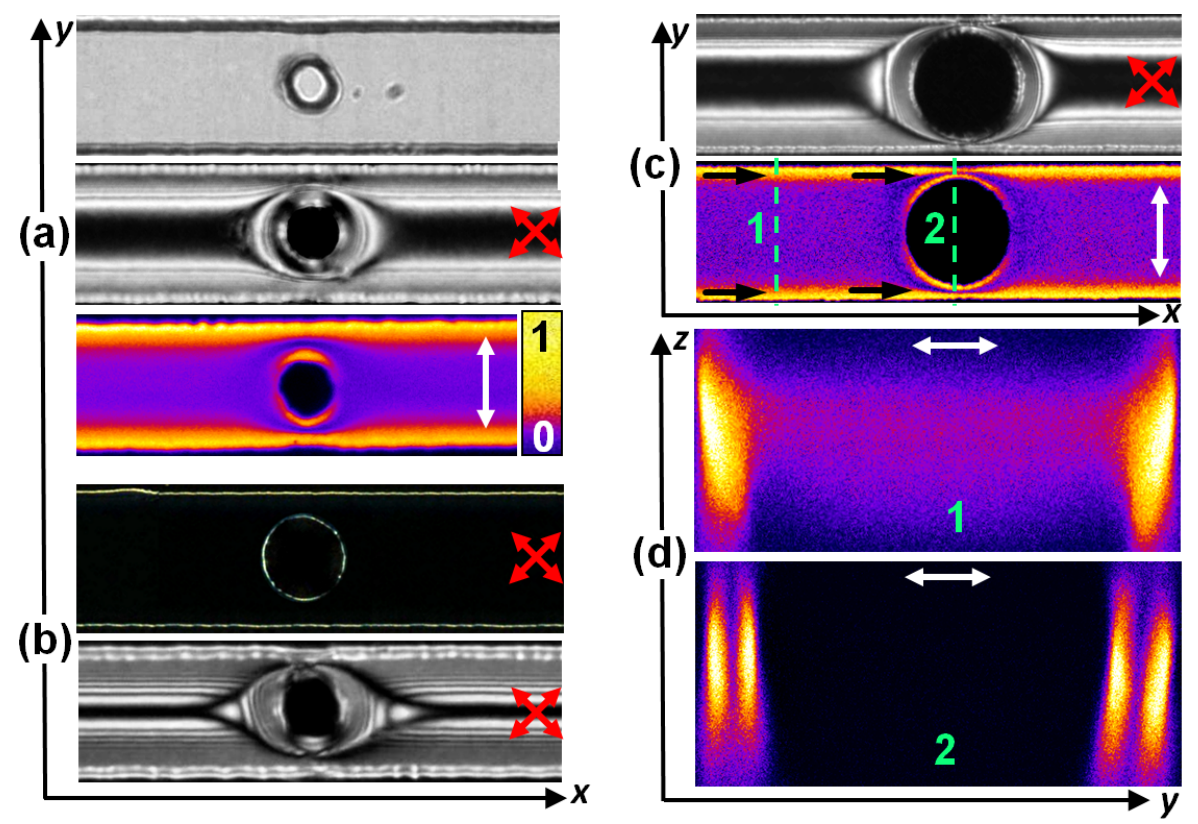

Figure 5.8: POM and FCPM imaging of the confined NLC around the micro-pillar. Polarized micrographs are indicated by a pair of crossed arrows (polarizers at $\pi / 4$ to the channel length). (a) From top: White light, polarized optical, and fluorescence confocal polarized micrographs (laser polarization at $\pi / 2$ to the channel length, indicated by the double-headed arrow) of a $(r, d, w)=(20,40,100) \mu \mathrm{m}$ channel. 0 and 1 on the FCPM intensity scale bar correspond to director orientations parallel and perpendicular to the laser polarization direction. (b) From top: POM images of a $(r, d, w)=(30,10,100)$ and $(r, d, w)=(20,40,50) \mu \mathrm{m}$ microchannel respectively. Note the reduction of the number of interference fringes in the shallow $(r, d, w)=$ $(30,10,100) \mu \mathrm{m}$ channel. (c) POM and FCPM images of $(r, d, w)=(30,40,100)$ microchannel.(d) FCPM imaging of the $y z$ cross-sections along the lines $\mathbf{1}$ and $\mathbf{2}$ in (c). The polarization of the excitation laser is shown with the double-headed arrow.

spread further to $\approx 75 \%$ (Fig. 5.8 ). The overall confinement-induced behaviour, discussed so far, is generally observed also in presence of multiple pillars within a homeotropic matrix.

The director field around the micro-pillar, reconstructed from the POM and FCPM data, is shown in Fig. 5.9a and b. At the confining walls and the micro-pillar, LC molecules anchored homeotropically. Consequently, the director is normal to the $x y$ plane away from the pillar, represented by black dots in Fig. 5.9p. In the vicinity of the side walls and the pillar, the director undergoes a transition from the in-plane to the out-of-plane orientation through the gradual bend deformation (indicated by the nail heads in Fig. 5.9p). Additionally, the director orientation around the micro-pillar preserves a rotational symmetry about its axis $(z)$. Two possible director orientations about the $y z$ plane - singular $+1 / 2$ defect loop (sharp pillar 


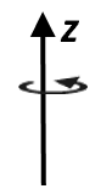

(a)
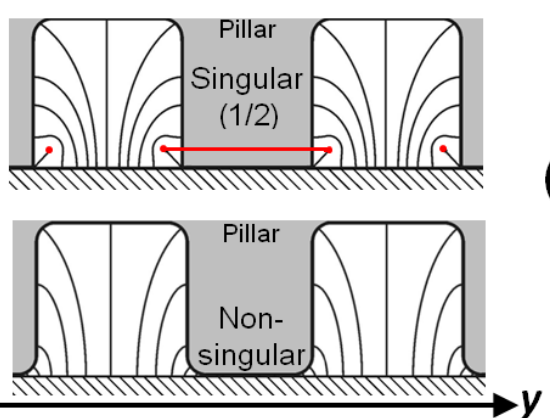

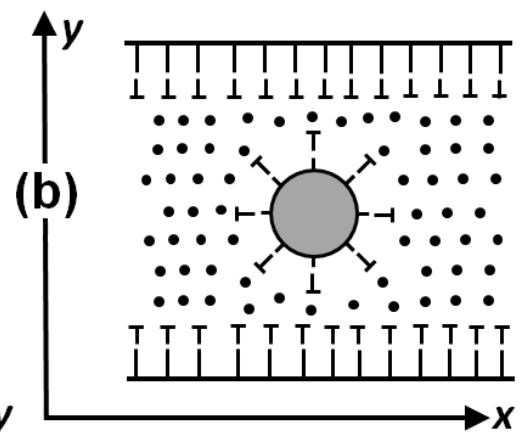

Figure 5.9: Director configuration around the micro-pillars. (a) Reconstruction of the director field from the POM and FCPM images. Hatched lines depict the glass surface. Cross-sectional view $(y z)$ through the pillar (shown in grey colour) shows two possible director orientations around it: singular $+1 / 2$ defect loop (top) or a non-singular director configuration (bottom). Both configurations possess symmetry about the cylinder $z$ axis. (b) Schematic nail representation of the director field on $x y$ plane. The nail heads indicate director tilted partially into the image plane.

base), and the continuous non-singular director field (rounded pillar base) - are shown in Fig. 5.9 a. Although symmetric projections of the above two cases are shown in Fig. 5.9a, asymmetric director configurations, as shown in Fig. 5.4, are also likely [195]. Typically in the experiments presented here, the non-singular configuration was observed long time after equilibration of 5CB from the isotropic to the nematic phase. The singular loop manifested itself typically at elevated temperatures (Fig. 5.10), under external perturbations, or due to pinning of the defect loop at surface irregularities.
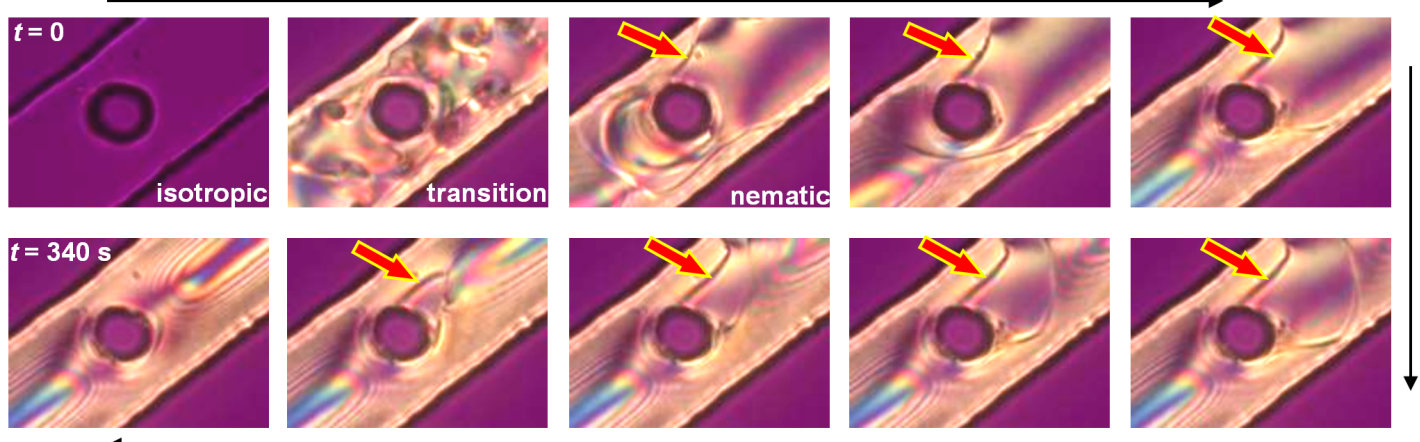

Figure 5.10: Defect loop around a micro-pillar $(r=25 \mu \mathrm{m})$ is observed through the sequence of optical micrographs, obtained during the transition of 5CB from isotropic to the nematic phase. Time proceeds along the black arrow. Different stages of the loop formation is pointed by the red arrow head on the micrographs. Time interval between the images is not uniform. 


\subsection{Microchannels with hybrid anchoring}

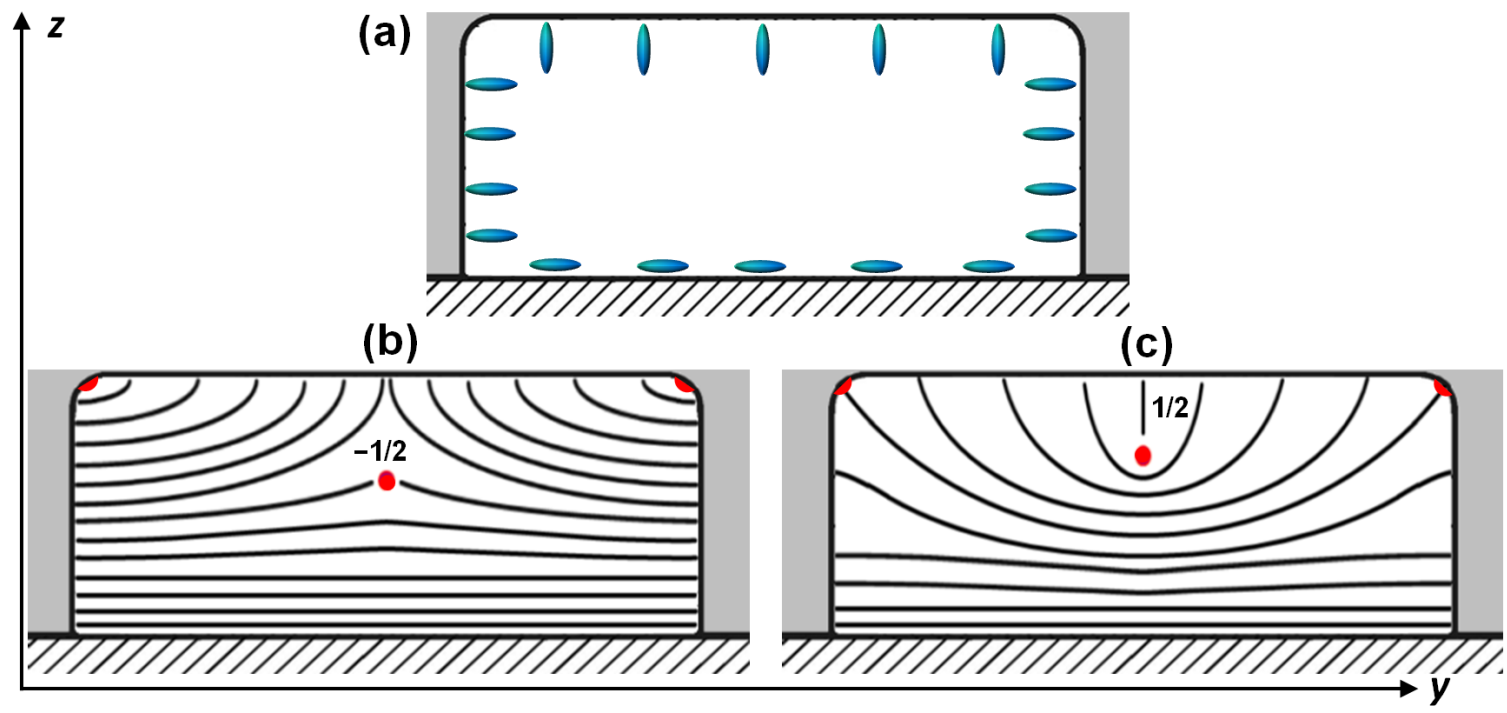

Figure 5.11: Microfluidic confinement for hybrid alignment. (a) Anchoring on the channel walls: On PDMS walls, the molecules orient perpendicular to the surface, and glass (bottom) supports planar anchoring orthogonal to the flow direction (perpendicular to the image plane). Two possible equilibrium director configurations, conserving the net topological charge, emerge: (a) Either a structure equivalent to topological rank $+1 / 4$ and a singular defect of rank $-1 / 2$, or (c) the the topological complement of the configuration shown in (b). These configurations however do not correspond to the minimum free energy state.

The practical realization of different possible hybrid alignments within microchannels was discussed in Chapter 4.3.4. In this section, we shall consider PDMS-glass microfluidic devices which possess homeotropic anchoring on the PDMS walls, and uniform planar anchoring on the glass surface. Most of the studies were conducted within $\approx 15 \mu \mathrm{m}$ deep channels. The channel width was varied typically between $50 \mu \mathrm{m}$ and $500 \mu \mathrm{m}$. The planar orientation on the glass surface was tailored for a range of director alignments relative to the channel length (flow direction). The discussions here will be confined only to the case of alignment perpendicular to the channel length. Figure 5.11 a shows a schematic representation of the surface anchoring on the microchannel walls. At room temperature, the LC equilibrated to the nematic phase, and the director field developed following the interplay of surface anchoring and longrange ordering [6], as depicted in Fig. 5.11b and c. At corners formed by two PDMS walls, the alignment encounters a situation of nonconformity, which is resolved through two possible elastic deformations of the director field. In topological terms, the deformations correspond 
82 Chapter 5. Nematic liquid crystals confined within a microfluidic device: Static case

(a)

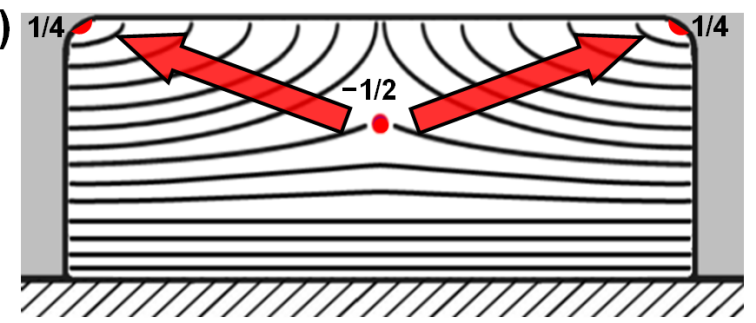

(b)

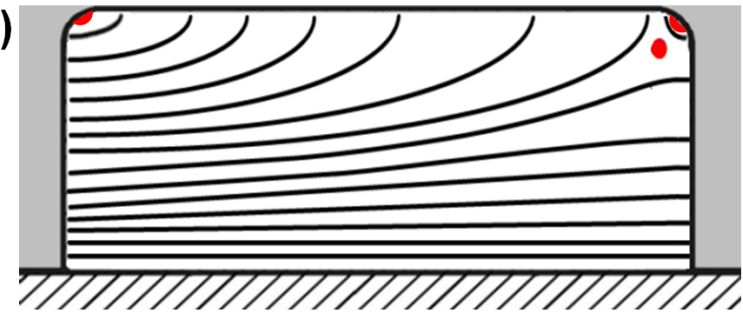

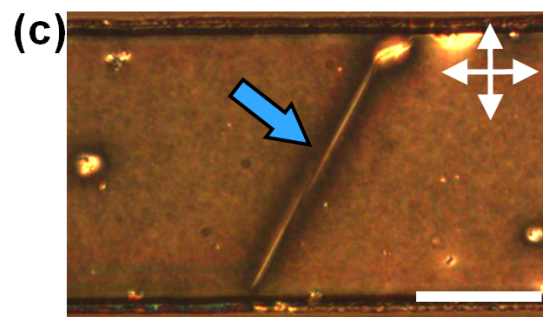

(d)

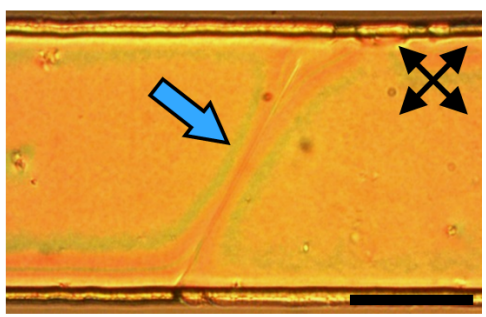

(e)

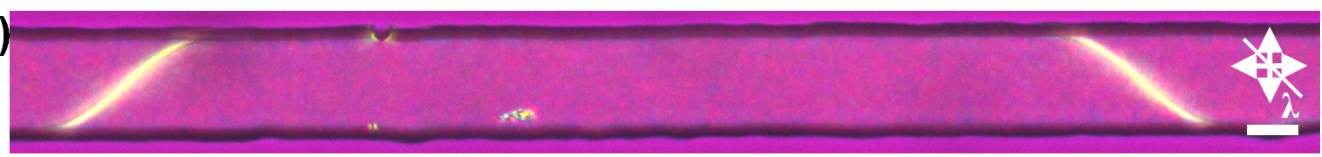

Figure 5.12: Equilibrium of the nematic director in hybrid alignment conditions. (a) The bulk disclination line experiences an attractive force due to the opposite topological charges at the corners. (b) The defect settles at the corner in equilibrium state. (c) - (e) Polarization micrographs showing cross-over of the disclination line from one side of the channel to the other. Multiple cross-overs were also observed (e). Scale bar: $50 \mu \mathrm{m}$.

to regions of topological rank $1 / 4$ at each corner. The net topological charge is nevertheless conserved through the development of a singular line defect, of opposite strength and rank $1 / 2$, within the nematic bulk. However, these configurations do not correspond to the state of minimum free energy.

As a consequence of a (logarithmic) attractive potential [76], defects of opposite topological charges approach each other (Fig. 5.12 $\mathrm{k}$ ). Hence, the semi-integer defect line settles at one of the corners (Fig. 5.12p). Using the relations of Dafermos [196] and Ericksen [13] for isotropic elasticity $\left(K=5.5 \times 10^{-12} \mathrm{pN}\right)$, the attractive force between the bulk disclination and each of the oppositely charged topological entity at a corner was estimated. The total energy $E_{12}^{\text {elastic }}$ and force of interaction $F_{12}^{\text {elastic }}$ between two disclinations is given by:

$$
\begin{aligned}
& E_{12}^{\text {elastic }}=2 \pi K s_{1} s_{2} \ln \left(\frac{r_{12}}{r_{c}}\right) \\
& F_{12}^{\text {elastic }}=2 \pi K\left(\frac{s_{1} s_{2}}{r_{12}}\right)
\end{aligned}
$$


Here, $s_{1}$ and $s_{2}$ are the topological ranks of the interacting defects, and $r_{12}$ is the separation between them. It needs is to be noted that the charge of the topological defects (positive or negative) has to be considered in the estimation of forces. In the present calculations, the interaction between the defects at the corners was neglected due to the large separation between them. The bulk disclination was thus found to be attracted with a force of $F_{\text {elastic }} \approx 4 \mu \mathrm{N} / \mathrm{m}$ at a separation of $1 \mu \mathrm{m}$ away from the corner. At the isotropic-to-nematic transition, the defect equilibrates with similar probability at either corner, resulting in a cross-over from one to the other side of the longitudinal confinement $(l \gg w)$, as observed clearly in polarization micrographs (Fig. 5.12k - e). Figure 5.12p shows the resulting director field in the vicinity of the cross-over. The tendency of the defects to dwell near the walls can be overcome, however, by using electric fields [184]. In Chapter 6, we shall look at an alternative method to shift the director equilibrium - by utilizing the viscous drag forces - in presence of a flow field. 



\section{Chapter 6}

\section{Flow of nematic liquid crystals in a microfluidic environment}

The flow of anisotropic liquid crystals in functionalized microchannels is uniquely distinct from its isotropic counterpart. The gamut of interesting phenomena presented in this chapter can be generalized as the effects of the surface-induced ordering on the flow behaviour, and the effects of flow on the surface-induced ordering of the LC phase. However, such a classification for the presented experiments is seldom true: The director and flow fields are allowed to evolve dynamically in these experiments following the coupling-mediated interplay of the elastic, surface, and viscous forces. In this chapter, we shall see how microfluidics of anisotropic liquid crystals provide attributes that are typically unobserved in isotropic fluidics. The numerical simulations for flow in linear homeotropic channels were carried out in collaboration with Miha Ravnik and Julia M Yeomans.

\subsection{Elastic, surface and viscous interactions on a microfluidic platform}

The advent of microfluidics [145] has opened up possibilities to study flows within micronsized confinements and past minute obstacles. It has evolved as the major technological platform on which bio-technology, material science and other related fields have seen a tremendous growth in the last decade [197-200]. In contrast to the flows at macro scales, flow within microchannels is fundamentally different due to the drastic reduction of the intertial effects, resulting in very low Reynolds numbers [201]. In general, the flow of Newtonian fluids in a 
microchannel is laminar. However, studies have shown that such a hydrodynamic regime may not be strict when a non-Newtonian fluid e.g. polymer solution [3] is flowed, or in presence of appropriate morphological patterning of the microchannel [202]. In particular, it has been recognized that microfluidic devices can be beneficial - or instrumental - in a much greater range of applications as one moves away from utilizing homogeneous liquids as the transport medium. The use of emulsions, where typically aqueous droplets are embedded in an immiscible carrier fluid such as oil, has become known as droplet-based microfluidics. The approach is now widely used in essentially all fields where microfluidic devices are employed [144]. Several methods have been proposed to control the motion and position of individual droplets or other cargo within the carrier fluid by means of external fields [203] and 'railed microfluidics' [204, 205]. The necessity of applying external fields and patterning grooves, however, imposes important limitations in terms of design possibilities and accessible parameter ranges.

The average orientation of the liquid crystal molecules, the director $(\vec{n})$, plays a decisive role in determining the rheological properties of the anisotropic liquid crystal [73]. The dynamics of liquid crystal flows, which are inherently complex due to the coupling between flow and orientational order [14, 15], lead to a variety of interesting phenomena like the generation of a transverse pressure gradient in Poiseuille flow [21], anomalous colloidal rheology [25], and the backflow mechanism [114]. The results in the present chapter further demonstrate that the use of an anisotropic fluid (liquid crystal) as the flowing matrix within microfluidic devices can induce effects distinct from those observed for an isotropic fluid (see (5), (6), and (7) on the publication list). By controlling the anchoring on the channel walls, the flow speed, and the channel aspect ratio $(w / d)$, different attributes of nematic flows were revealed, consequently leading to potential applications based on lab-on-a-chip.

We shall continue our discussions from Chapter 5 and introduce a flow field to perturb the nematic 5CB confined within the functionalized microfluidic devices. We shall initially look at the bulk rheology of the nematic 5CB, by allowing the flow to develop within differently functionalized channels. The flow was induced by applying a pressure difference between the inlet and the outlet (Section 6.2). Subsequent experiments were carried out by flowing nematic $5 \mathrm{CB}$ at a constant rate using a precision pump. The first set of such experiments is aimed to study the flow-induced evolution of topological defects within channels possessing degenerate planar anchoring (Section 6.3). In Section 6.4, the flow of a nematic liquid crystal in a homeotropic microchannel is investigated and discussed. Using experiments and numerical modeling, the flow and the LC orientation coupling is conclusively demonstrated. The flowdirector coupling was subsequently utilized to devise a non-intrusive opto-fluidic velocimetry 
technique on a microfluidic platform. The flow-induced birefringence was analyzed within a diverging channel, which, in a single step and in real time, determined the flow speed and the director deformation. Within homeotropic confinements, the study was further extended to nematic flows past micron-sized obstacles placed in the flow path (Section 6.5). Flow through microchannels supporting hybrid alignment is investigated in Section 6.6. Interestingly, the director field in this case resulted in the creation (and stabilization) of a disclination line that extended through the entire length of the channel $(\geq 2 \mathrm{~cm})$. By tailoring the surface anchoring in an appropriate manner, the line was guided at a flow bifurcation. Finally, in Section 6.7 we shall discuss the nematic flows at high Ericksen numbers. The flow undergoes a defectmediated transition to a chaotic regime, which was found to depend on the surface properties and the depth of the microchannel.

\subsection{Nematic flow due to pressure gradient}

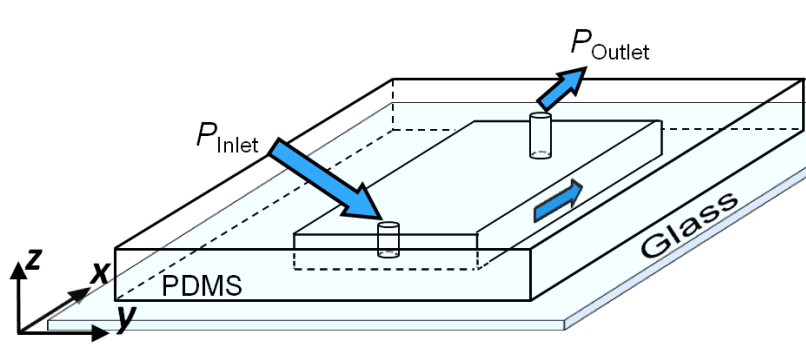

(a) (b)

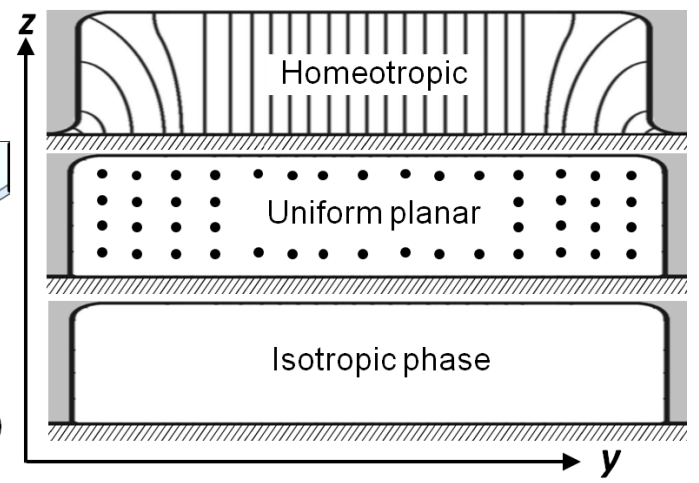

Figure 6.1: Pressure-driven nematic flow. (a) Schematic of the microchannel, flow is along $x$ direction. (b) Relative orientation of the initial director equilibrium and flow direction: homeotropic $(\vec{v} \perp \vec{n}, \nabla \mathrm{v} \| \vec{n})$, uniform planar $(\vec{v} \| \vec{n})$, and flow in isotropic phase.

The flow of nematic 5CB, induced by the application of a pressure gradient, provides us insights into the rheological behaviour of the material. To this effect, the microchannel inlet was connected to a pressure controller (Chapter 3.3.2), and the outlet was left at atmospheric pressure. The outlet tubing was was immersed within a $5 \mathrm{CB}$ sink placed on a sensitive mass balance, which measured the total mass of 5CB that flowed through the channel with a precision of $\pm 100 \mu \mathrm{g}$. For ensuring a reduced influence of the side walls, channels of very high 


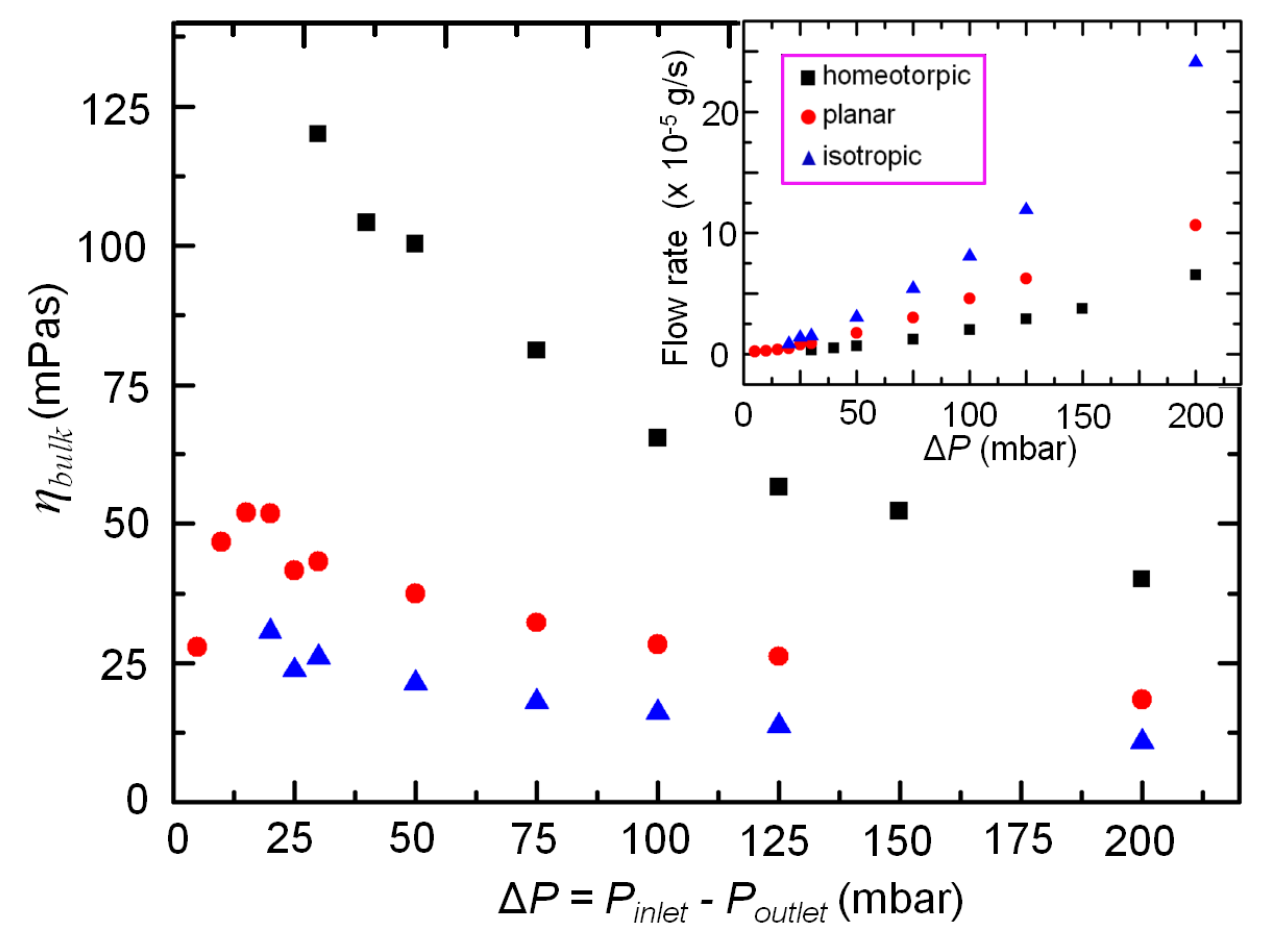

Figure 6.2: Average viscosity of 5CB measured using pressure-driven flow for homeotropic $(\vec{v} \perp \vec{n}, \nabla \mathrm{v} \| \vec{n})$, uniform planar $(\vec{v} \| \vec{n})$, and isotropic cases, shown respectively by the black squares, red dots, and blue triangles. The measurements for the homeotropic and the uniform planar anchoring cases were done at room temperature. The inset plots the measured mass flow rate as a function of applied pressure difference.

aspect ratio $(A R=w / d \approx 133)$, with $w \approx 2 \mathrm{~mm}$ and $d \approx 15 \mu \mathrm{m}$, were considered for the measurements. Three different flow cases were measured, based on the initial director equilibrium within the channels: $\vec{v} \perp \vec{n}$ and $\nabla \mathrm{v} \| \vec{n}$ (initially homeotropic), $\vec{v} \| \vec{n}$ (initially parallel to the channel length), and for $5 \mathrm{CB}$ in isotropic phase. Figure 6.1 summarizes the experimental cases considered.

Figure 6.2 plots the experimental estimation of the effective dynamic viscosity of 5CB as a function of applied pressure difference $\Delta P=P_{\text {inlet }}-P_{\text {outlet }}$. The effective viscosities were calculated by measuring the mass flow rate of $5 \mathrm{CB}$ at different pressure differences:

$$
\eta=\frac{w d^{3}}{12 l} \rho\left(\frac{\Delta P}{\dot{m}}\right)
$$

Here, $w, d$, and $l \approx 4 \mathrm{~cm}$ denote the channel dimensions. $\rho, \Delta P$, and $\dot{m}$ signify 5CB density, applied pressure difference, and the measured mass flow rate, respectively. Two different 
values of 5CB density were considered: in nematic phase $\rho=1025 \mathrm{~kg} / \mathrm{m}^{3}$, and in isotropic phase $\rho=1013 \mathrm{~kg} / \mathrm{m}^{3}$ [136]. The expansion of PDMS at $\approx 35^{\circ} \mathrm{C}$ (isotropic phase) was neglected in the calculations. Additionally, the pressure drop at the inlet tubing of the channel $(\approx 0.3$ mbar at maximum flow rates), was neglected in determining the actual pressure at the inlet port.

As seen clearly in Fig. 6.2, the effective viscosity of 5CB depends not only on the thermodynamic phase, but also on the nature of the boundary conditions prevalent. The flow was observed to experience a significantly higher viscous resistance under homeotropic conditions $\left(\eta_{\text {homeotropic }}\right)$, compared to the flow where the velocity and initial director were mutually parallel $\left(\eta_{\text {planar, },}\right)$. Consequently, for a similar pressure difference between the inlet and the outlet ports, nematic 5CB flowed slower in the homeotropic channel than in the channel with uniform planar boundary conditions. It is thus logical to expect that the viscosity $\eta_{\text {planar }, \perp}$ for the initial configuration $\vec{v} \perp \vec{n}-\nabla \mathrm{v} \perp \vec{n}$, shall lie intermediate between the $\eta_{\text {homeotropic }}$ and $\eta_{\text {planar,\| }}$ values. As the applied pressure was increased, the difference of the viscosity values reduced. It might be worthwhile to mention here that the bulk nematic 5CB exhibits a shear thinning behaviour [1]. The present experiments additionally show that this behaviour holds true for disparate anchoring conditions, and also in isotropic phase (Fig. 6.2, inset). Moreover, the variation of the viscosity within the planar microchannel shows an initial increase, before going down at higher applied pressures. This is attributed to the director reorientation. On starting the flow, the director reorients slightly away from the initial state (parallel relative to the flow direction), due to the interplay of the viscous and the elastic torques. Consequently, the effective viscosity has an additional contribution from the Miesowicz viscosity $\eta_{3}$ (Chapter 2.7.2). The reorientation continues till the viscous torque vanishes at the critical Leslie angle [76]. Thereafter, the viscosity reduces monotonically, as in the case of the isotropic and homeotropic flows. The discussed anisotropic attributes offer interesting avenues, especially the flow-director coupling can be utilized to devise novel applications based on LC microfluidics. For instance, spatially modulated flow fields can be created within microchannels by incorporating composite boundary conditions, as the one shown in Fig. 6.3

Further experiments on nematic flows demonstrated the reduction of the flow resistance at elevated temperatures $\left(\approx 35^{\circ} \mathrm{C}\right)$. This is shown in Fig. 6.4. The temperature-induced reduction of the effective viscosity is significant considering that in an isotropic fluid the corresponding variation is rather marginal. In the present experiments, upon transition to isotropic phase, the mass flow rate enhanced by $\approx 100 \%$ in planar case (at 120 mbar), and by $200 \%$ in homeotropic case (165 mbar). The distinct flow behaviour of nematic 5CB can be related 


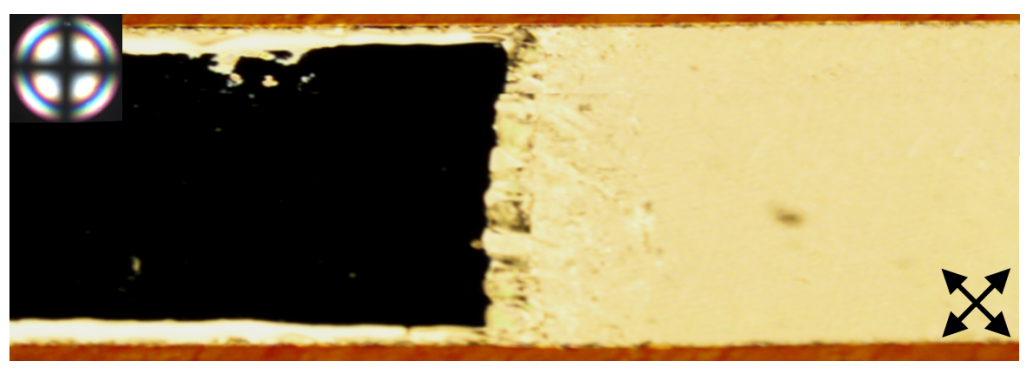

Figure 6.3: Polarized micrograph of a microchannel possessing composite boundary conditions. Shown here: the left half is homeotropic, and right half is uniform planar. Such microchannels can be utilized to produce micro-flows with spatially modulated velocities.

to the temperature-induced director alignment over and above the usual reduction of material viscosity with temperature. As the temperature goes up, the nematic molecules show a transition typically from homeotropic to planar [206]. In the present context, such an occurrence reduces the viscosity, as the molecules are now increasingly aligned along the flow direction. However, as the temperature increases further, the director alignment reduces abruptly close to the transition temperature, marked by increased viscosity. The flow phenomena close to the transition temperature were investigated experimentally by Gähwiller [207], and the phenomenological relations were given by W. Helfrich [208, 209]. Just below the transition temperature, where the nematic order is lowest, the Leslie angle (equation 2.51) increases. The flow-aligned molecules, owing to the reduced order, have an increased degree of freedom with respect to the aligned orientation. At this stage, the molecules can even rotate about their short axes. Consequently, the molecules behave as elongated structures under shear flow. The long axes of such molecules align preferentially at an angle of $45^{\circ}$ between the direction of flow and the velocity gradient [210]. The situation however changes as the temperature is reduced: The degree of order becomes larger again, and the effective viscosity is now determined by the relative orientation between the director and the flow. Some interesting observations on the rheology of nematic 5CB under the application of an electric field have been reported by K. Negita [211]. Investigations for a more detailed understanding of the flow behaviour at the nematic-to-isotropic transition are underway. The characteristic dependence of the nematic flows on boundary and temperature conditions can be potentially applied for flow-field and particle manipulations. 


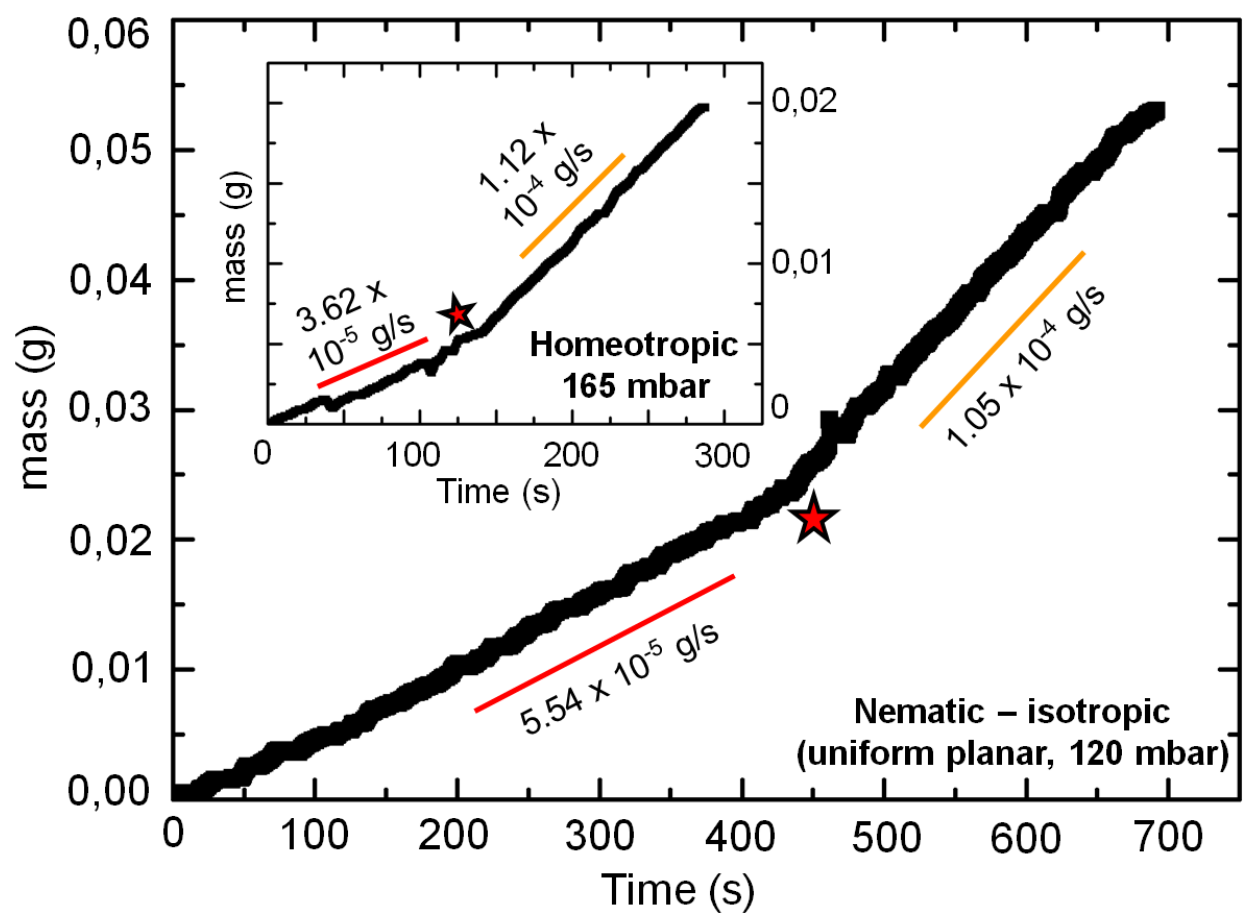

Figure 6.4: Flow before and after nematic-to-isotropic transition, indicated by the star symbol. The mass flow rate increased by $\approx 100 \%$ in planar case (at 120 mbar), and by $200 \%$ in homeotropic case (165 mbar).

\subsection{Nematic flow in a degenerate planar microchannel}

The study of nematic flows within microchannels possessing degenerate planar surface anchoring teems with topological defect structures of different forms and nature. On one hand, this is optically appealing, on the other hand, the controlled creation of the defects within such confinements is rather challenging. However, over a large number of experiments, the general statistics of the defect evolution could be resolved. Depending on the channel dimensions and the flow rates, the formation of different textures and topological defect structures was observed and studied using polarizing optical microscopy and fluorescence confocal polarizing microscopy. The observed structures comprise $\pi$-walls, disclination lines pinned to the channel walls, disclination lines with one pinned and one freely suspended end, and disclination loops freely flowing in a chaotic manner. Although the defect analyses appeared daunting at first glance, the phenomena observed within these channels provided the motivation and the direction for a number of subsequent experiments in which defects could be generated and 


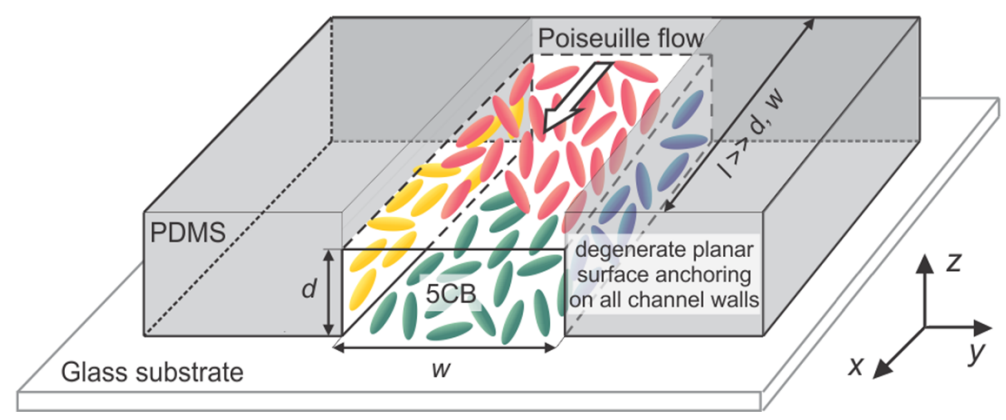

Figure 6.5: Schematic of Poiseuille flow within a microchannel possessing degenerate planar anchoring.

guided with good control. Such defects were applied for guiding the transport of colloidal particles, quantifying the interaction of defects and colloids, or whole-field estimation of the flow-induced director field.

Liquid crystal textures in absence of any external field were discussed in Chapter 2.6. In this present section we shall discuss defects which were generated and stabilized (or destabilized) by a nematic flow within degenerate micro-confinements (Fig. 6.5). The appearance of the four different nematic textures depended on the parameter space of flow velocity and channel depth. Investigations into the morphology of the defects revealed interesting director patterns imprinted by the flow field, which could be manipulated using pressure changes. Owing to the degenerate surface conditions, the molecules failed to orient along any preferred direction, thereby giving rise to topological singularities. When the channel is filled up with $5 \mathrm{CB}$ in the nematic phase, these defects acted as the starting points of defect lines connecting two equal topological charges on top and bottom of the channel. Figure 6.6a schematically shows the initial stages of alignment of such a disclination line under flow. The stretched line slides on the confining surfaces and starts to move downstream, simultaneously reducing its length. Its length reduction, favoured by the free energy minimization and assisted by the viscous drag, results into a straight vertical disclination. This process takes place in tens of milliseconds.

By varying the Ericksen number (flow velocity $v$ and/or channel depth $d$ ), different forms of flow and confinement induced textures evolved. High Ericksen numbers suggest that these textures emerge under non-equilibrium conditions. For $E r \leq 900(d<10 \mu \mathrm{m}, 0.2 \mathrm{~mm} / \mathrm{s}$ $<v<15 \mathrm{~mm} / \mathrm{s}$ ), the director field in the bulk undergoes a spontaneous bend deformation in the azimuthal plane creating the $\pi$-walls [48, 212-214] (also referred to as planar soliton textures), 
(a)
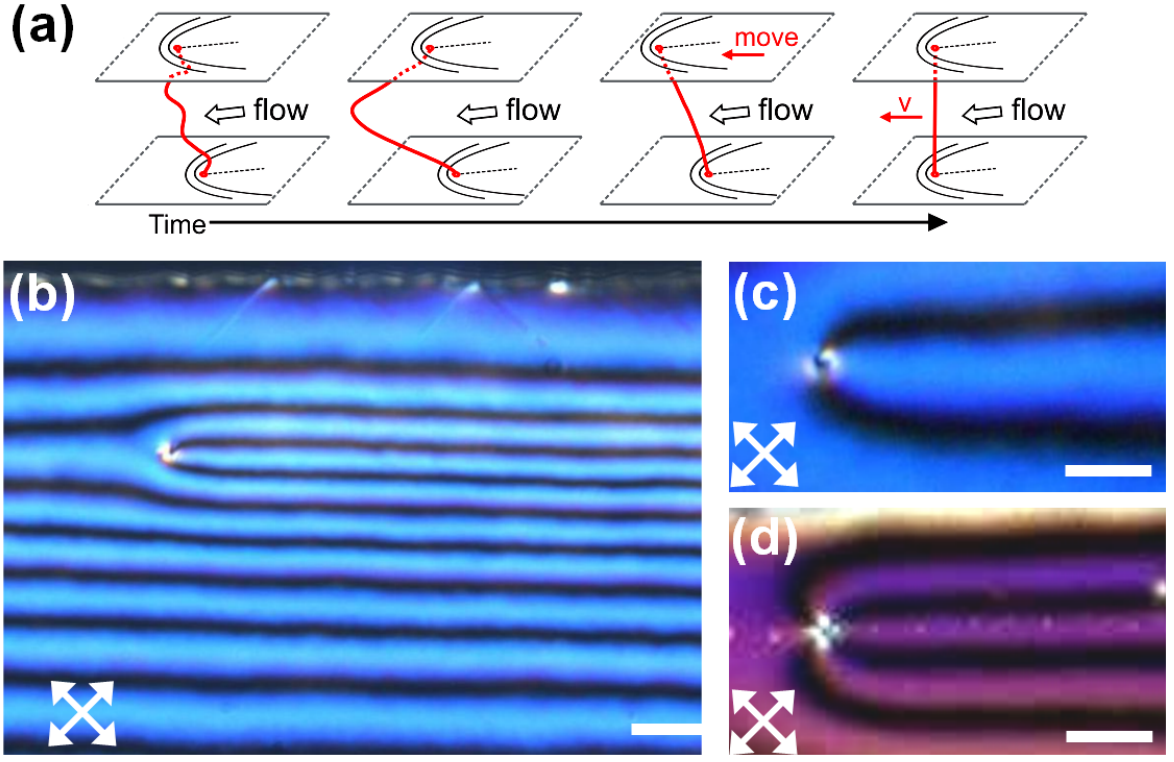

Figure 6.6: Formation of a $\pi$-wall due to the nematic flow. (a) Flow-induced creation of $\pi$-walls in microchannels with degenerate planar anchoring. The defect line evolves spontaneously, which over time, reduces in length and stabilizes by aligning vertically within the channel. The defect formation and subsequent alignment takes place in tens of milliseconds. (b) Polarization optical micrograph of parallel set of $\pi$-walls formed by Poiseuille flow. Isolated $\pi$-wall structures: (c) semi-integer, (d) integer $\pi$-wall. Flow direction is from right to left. Scale bar: $5 \mu \mathrm{m}$.

shown in Fig. 6.6p - d. This leads to an assortment of alternating dark and bright stripes, and is recognized as a set of parallel extinction branches between crossed polarizers. Such a set of parallel stripes is created by sliding of vertical disclination lines, which are stabilized by confinement, only along the channel depth. This is in contrast to the observations within circular and square microchannels $(d=w \leq 10 \mu \mathrm{m})$, where the disclinations were stabilized also across the channel width. Using a rectangular microchannel $(w>3 d)$, the disclinations were thus biased to stabilize in the vertical direction.

At higher values of the Ericksen number $(900<E r<1250)$, the disclination line at the leading end of the $\pi$-wall got depinned from the glass surface. The flow field consequently oriented the hitherto vertical defect line horizontally along the flow direction and eventually got repinned on the PDMS surface downstream. This was observed either at a relatively small flow rate $(\approx 6 \mathrm{~mm} / \mathrm{s})$ and larger channel depth $(10 \mu \mathrm{m}<d<20 \mu \mathrm{m})$ or at higher flow rate $(\approx 18 \mathrm{~mm} / \mathrm{s})$ and smaller channel depth $(\approx 10 \mu \mathrm{m})$. Due to the role played by the channel 


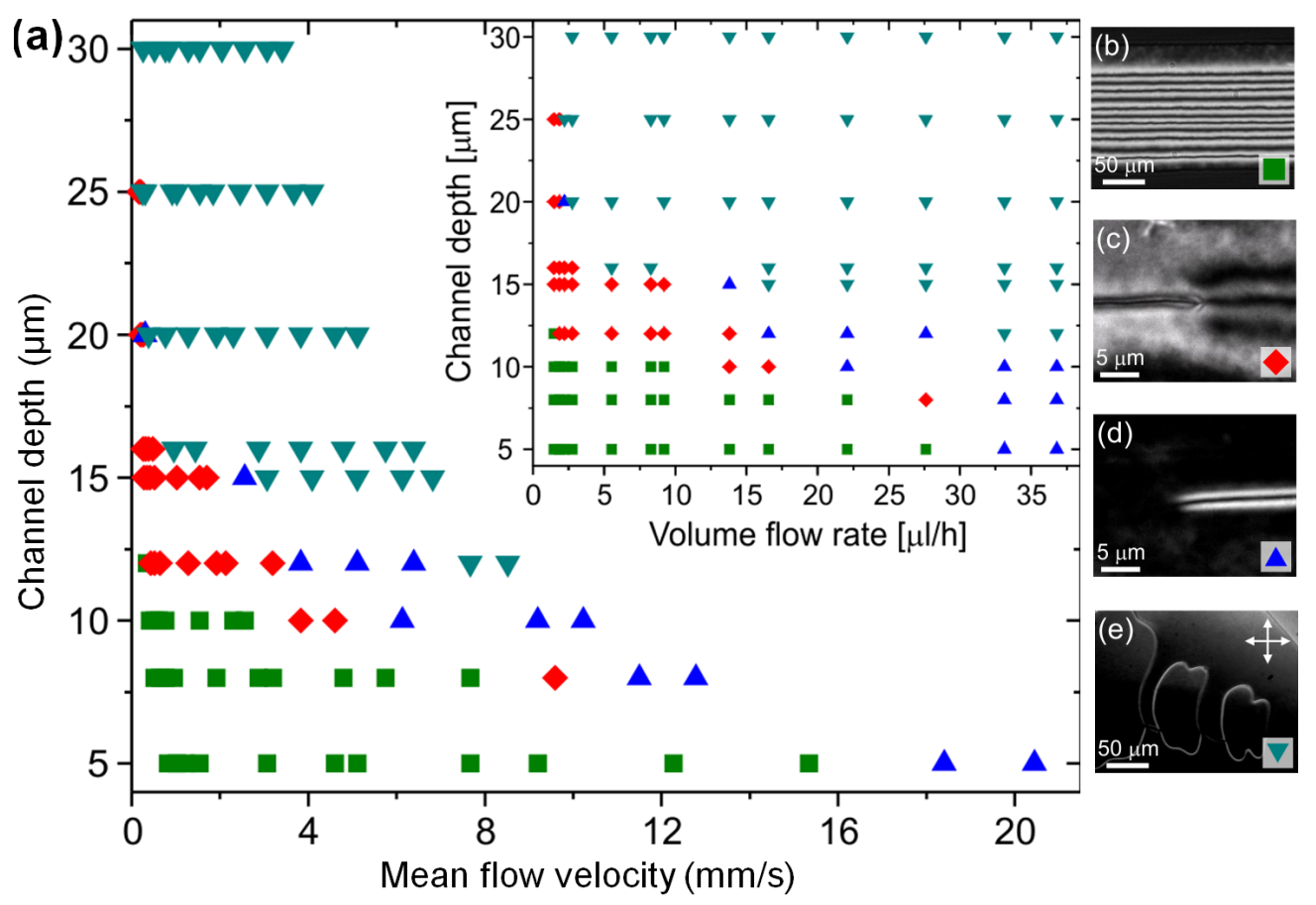

Figure 6.7: Flow-induced textures in parameter space of velocity and channel depth. (a) Occurrence of nematic textures on variation of flow velocity and channel depth: (b) $\pi$-walls, (c) $\pi$-wall with surface-stabilized disclination, (d) flow-stabilized disclination and (e) disclination loops in chaotic regime. A combination of small velocity and low channel depth, i. e., low Ericksen number, results in the creation of $\pi$-walls.

surface, we designate these textures as surface-stabilized disclinations, presented in Fig. 6.7k.

On further increment of $E r>1300$, the surface-stabilized disclinations transform to the third kind of flow-induced defect structures. These are disclination lines pinned only at one end. The other end exists downstream in the channel, stretched freely in the bulk of the flowing NLC. Figure 6.7d shows the polarized micrograph of the flow-stabilized disclination. In contrast to the $\pi$-walls, these structures are distinctly visible also without polarizers. Moreover, the length of these lines depends directly on the mean flow velocity and they completely disappear in the absence of the flow.

If $E r$ is increased further, typically above 1800 , the free end of the disclination oscillates about a mean position, before the system enters a chaotic regime where disclinations flow freely (Fig. 6.7e). The flow velocity required for transition between the textures reduces with the increase of the channel depth. Beyond $d \approx 25 \mu \mathrm{m}$, the textures directly enter the chaotic regime, even at small flow rates $(\approx 25 \mu \mathrm{m} / \mathrm{s})$. The chaotic regime is characterized by numerous 
freely flowing disclinations, both straight lines and loops which vigorously interact through intertwining, annihilating and crossing each other. In Fig. 6.7 $\mathrm{A}$, the occurrence of the discussed textures in the parameter space of channel depth and flow velocity is summarized. The sequence of the nematic textures in Fig. 6.7b - e occurs by increasing the Ericksen number.

For a thin nematic film on a surface possessing degenerate planar surface anchoring, typically the Schlieren textures comprising topological defects of integer $( \pm 1)$ and half-integer $( \pm 1 / 2)$ strengths are created [6]. Depending upon topological charge, either four or two dark brushes are observed between crossed polarizers. Disclination lines connecting the point defects of equal topological charge are generated spontaneously when the confining surfaces are brought in close proximity [134]. The nucleation and subsequent stabilization of such defect lines are additionally assisted by irregularities on the confining surfaces and by the presence of dispersed impurities.

\section{$\pi$-walls}

Under present surface and confinement conditions, the topological defect lines are randomly created when the nematic flow commences and fills up the microchannel. These defects are optically similar to static Schlieren textures in thin nematic films, however, when driven downstream by the flow, they leave behind a parallel set of dark and bright stripes, manifested between crossed polarizers. The generation of the leading disclination is not controlled, however, the number of stripes left behind is determined by the topological charge of the defect. Since the dark stripes span over a certain width, typically $\approx 4 \mu \mathrm{m}$ centre-to-centre, the number of stripes saturates when the entire width of the channel is occupied (see Fig. 6.7b). The velocity of the advancing end of the $\pi$-walls corresponds to $40 \%$ of the local flow velocity. This mechanism leads to formation of parallel $\pi$-walls extending up to several hundred micrometres in length downstream in the microchannel. Similar textures, termed as linear and planar solitons [48, 212, 213], have earlier been studied in superfluid phases of ${ }^{3} \mathrm{He}$ under external magnetic field and in hybrid nematic films [215].

Firstly, we shall focus on $\pi$-walls generated by integer defect lines. They are observed more scarcely, compared to their semi-integer counterparts, and occur only in very shallow channels $(d \approx 5 \mu \mathrm{m})$. Although, integer defects are expected to be energetically unstable, the non-equilibrium situation, and the severe confinement conditions appear to assist in stabilizing them. Stabilized integer disclinations have also been observed in thin $(\leq 5 \mu \mathrm{m})$ LC polymer films [216], where they tend to transform into more stable half-integer disclinations when the 

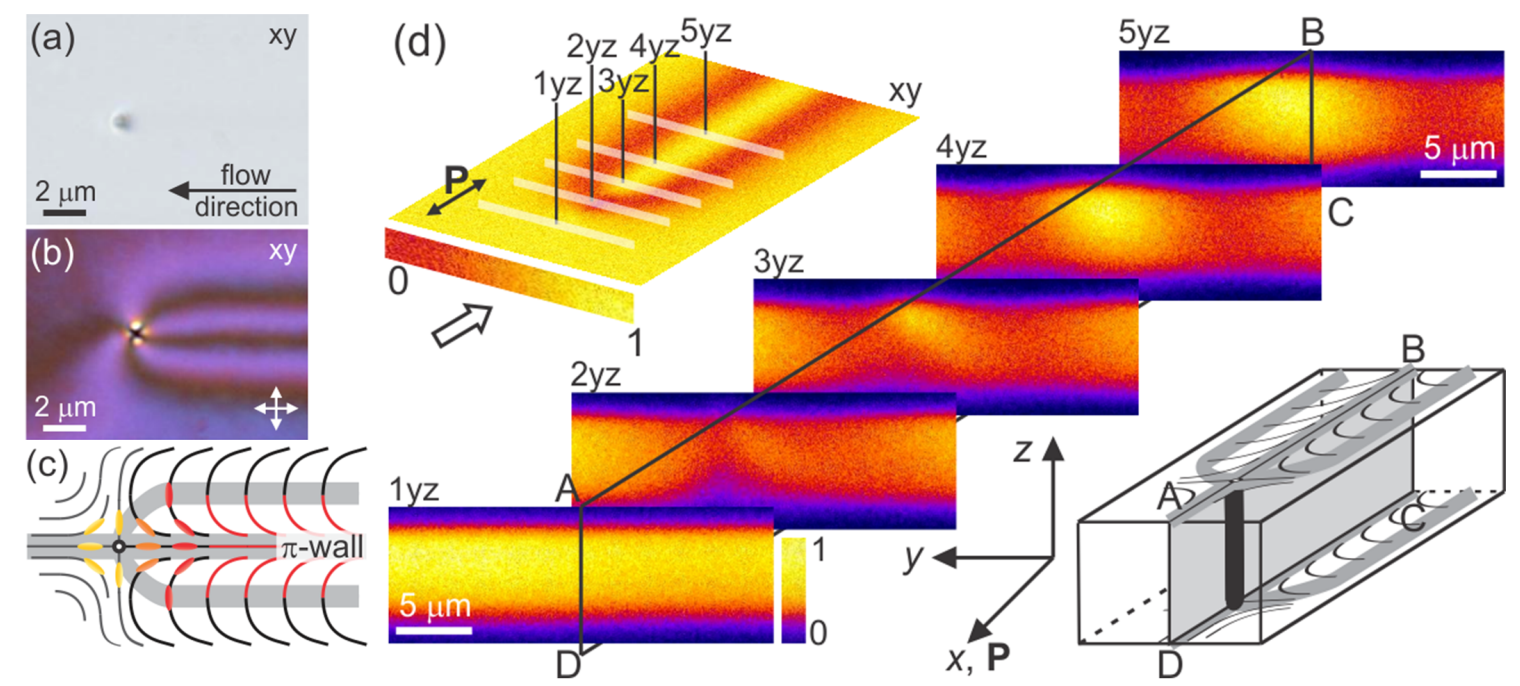

Figure 6.8: Morphology of a $\pi$-wall created from hyperbolic -1 disclination line. (a) Unpolarized micrograph showing the dark core of the defect at the leading end of $\pi$-wall. (b) Polarized optical micrograph shows alternate dark and bright regions which correspond to the molecular orientation in the vicinity of the defect core. (c) Schematic representation of the director profile in $x y$ plane. Red coloured molecules undergo one $\pi$-turn from the top to the bottom dark stripe, constructing the basic segment of the $\pi$-wall. (d) FCPM micrograph in $x y$ plane. FCPM image series in the $y z$ plane on traversing through $\pi$-wall from $1 y z$ plane to $5 y z$ plane. Combination of POM and FCPM micrographs provides the spatial morphology of a $\pi$-wall in two orthogonal planes.

film thickness was increased. These observations qualitatively resemble the ones in the present case. Under unpolarized light, only the leading defect core, which scatters light, is visible (Fig. 6.8a). Between crossed polarizers, four dark stripes originating from the defect core, which correspond to molecular orientations either parallel or perpendicular to the polarizers, are distinguished in Fig. 6.8 b. On rotating the sample between crossed polarizers, the sign of the topological defect at the leading end can be ascertained. Based on the POM micrographs, an in-plane director profile is schematically suggested in Fig. 6.8 $\mathrm{k}$. The molecular alignment behind the defect core, spanning between alternate dark stripes, undergoes an angular turn of $180^{\circ}$, as shown by the red curves in Fig. 6.8k. In analogy to the Néel wall [45], where magnetization undergoes an in-plane rotation by $\pi$, we name this bend deformation of the director field a $\pi$-wall.

In Fig. 6.8d, the $x y$ FCPM scan of the mid-plane is presented. The fluorescence intensity distribution, where the high intensity region is confined between two low intensity regions, 

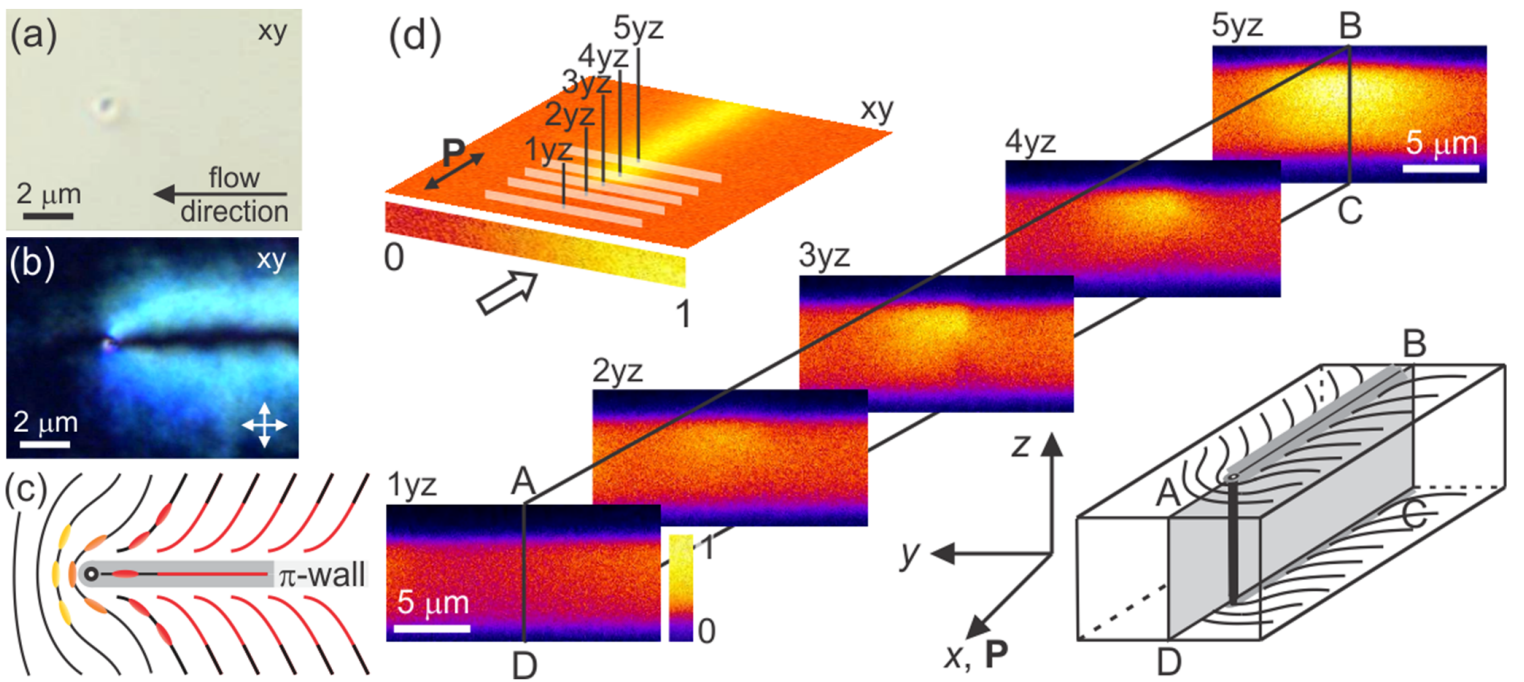

Figure 6.9: Morphology of a $\pi$-wall created from $+1 / 2$ disclination line. (a) Unpolarized micrograph. The dark point indicates the light scattering from the disclination line at the leading end of the $\pi$-wall. (b) Polarized optical micrograph shows alternate dark and bright regions which correspond to the molecular orientation in the vicinity of the defect core. (c) Schematic representation of the director profile in $x y$ plane. Red coloured molecules indicate one $\pi$-turn from top to the bottom dark stripe, constructing the $\pi$-wall. The red curves thereafter indicate the continuous $\pi$-wall structure along the channel length. (d) FCPM micrograph in $x y$ plane. FCPM image series in the $y z$ plane on traversing through $\pi$-wall from $1 y z$ plane to $5 y z$ plane. Combination of POM and FCPM micrographs gives the spatial morphology of $\pi$-wall in two orthogonal planes. The colour bars indicate the angular distribution of molecular orientations with respect to the polarization of the scanning laser beam.

supports the proposed molecular orientations inside the $\pi$-wall texture in Fig. 6.8c. Additional laser scans, performed at different $y z$ planes (1yz to $5 y z$ in Fig. 6.8 d), by traversing along $x$ coordinate, confirm that the $\pi$-wall uniformly stacks up from top to the bottom of the channel. The intermediate intensities (between 0 and 1 in the intensity bar), which indicate a continuous bend deformation of the director, demonstrate that the $5 \mathrm{CB}$ molecules gradually turn from the central flow-aligned region to the peripheral $\pi$-wall areas possessing alignment perpendicular to the flow. It appears that the $\pi$-wall stacks are held together by a leading disclination line, however, the precise structure of this line is beyond the reach of FCPM experiments. Additional numerical calculations are needed to address this detail. Nevertheless, combining the POM and FCPM data, we conclude that the director profile of the $\pi$-wall confined in two orthogonal planes is in agreement with the schematic illustration in Fig. 6.8 d. 
In addition, we observe morphologically similar $\pi$-walls created by the impact of flow on half-integer disclination lines. Such sets of $\pi$-walls, in contrast to the ones arising from integer disclinations, are more abundant, and evolve also in deeper channels (up to $\approx 10 \mu \mathrm{m}$ ). In the presence of flow, both integer and half-integer defect lines exhibit comparable temporal stability. After termination of the flow, the disclinations of half-integer strength are stable for days, while the integer disclination lines collapse sooner, in agreement with their high energetic cost. Figure 6.9 shows an example of a $\pi$-wall created by a $+1 / 2$ disclination line. Between crossed polarizers, one can observe a single extinction stripe arising from the defect core, shown in Fig. 6.9p. On rotating the polarizers by 45 degrees, the single dark stripe is replaced by a bright stripe with two surrounding dark stripes. These observations lead us to the schematic representation of the in-plane director profile, presented in Fig. 6.9 $\mathrm{c}$. Since the molecules undergo a $\pi / 2$ turn which is symmetric about the extinction stripe, another $\pi$-wall texture is recognized and indicated by the red curves. Further, FCPM studies were performed in the $x y$ and $y z$ planes to confirm the suggested director field. The intensity distribution in Fig. $6.9 \mathrm{~d}$ shows clearly a bright region, where molecular orientations coincide with the laser beam polarization, directly in correlation with the extinction stripe in Fig. 6.9 b. The $y z$ crosssections resolve the central region of the texture. Owing to the minuscule diameter of the half-integer defect line $(<100 \mathrm{~nm})$, there is no observable change in the intensity at the point of entry to the $\pi$-wall. However, further into the $\pi$-wall, the intensity contrast enhances as in Fig. 6.8d (5yz plane). The following experiments confirm the existence of $+1 / 2$ disclination line. Therefore, we conclude that the director profile in this case matches with the schematic representation in Fig. $6.9 \mathrm{~d}$.

The disclination lines and the attached $\pi$-walls exhibit significant morphological stability both under flow and long time after the flow termination $(\approx 36 \mathrm{~h})$. This stability can be attributed to the vertical confinement, and to the surface memory effects [217]. The $\pi$-wall system can be generally destabilized at sufficiently high flow velocity. The viscous pull on the disclination depins the leading disclination usually from the glass surface of the microchannel. Both integer and half-integer disclinations can depin from either one or both of the channel surfaces and get displaced by the flow.

When the flow is restarted after the textures were stabilized, the $\pi$-walls' length can be manipulated by varying the flow rate in a specific direction. Choosing between infusion or withdrawal flow, the leading end of the $\pi$-wall was manipulated along the downstream or upstream directions, respectively. Figures 6.10a and 6.10p show manipulation of an existing $\pi$-wall set, formed by an integer and a half-integer disclination line. When the disclination 

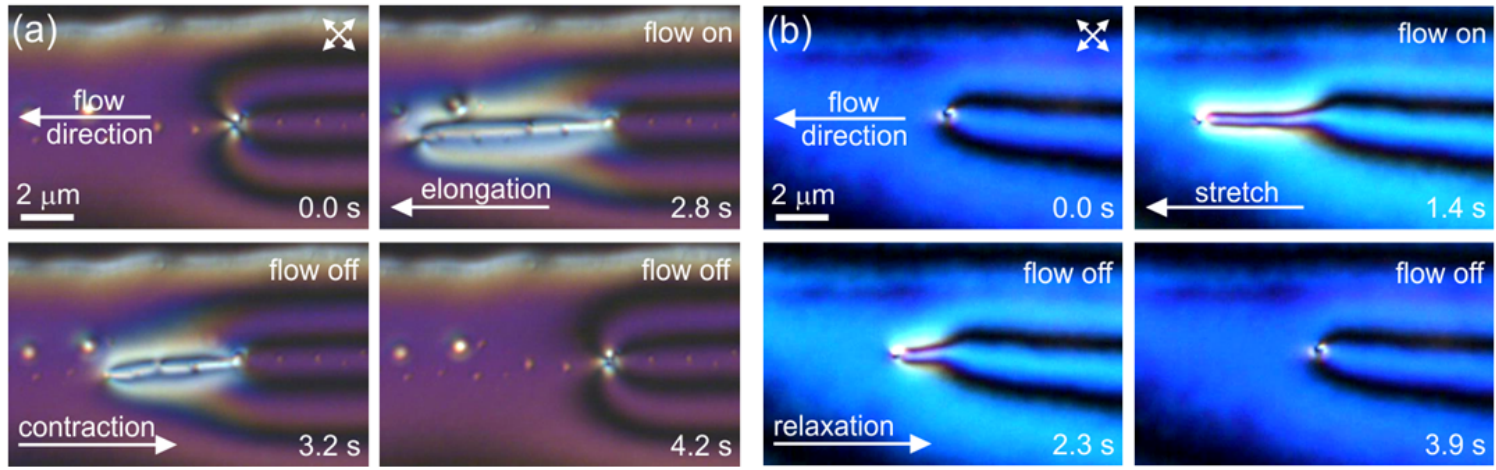

Figure 6.10: Manipulation of stable $\pi$-wall set using an infusion flow field. Local flow velocity corresponds to $480 \mu \mathrm{m} / \mathrm{s}$. (a) Elongation of a hyperbolic -1 disclination line when flow is turned on, with one end of the disclination is depinned and displaced, indicated by the four black stripes at both ends of the elongated line. On turning the flow off, the disclination contracts back to its vertical position. (b) Stretching of $+1 / 2$ defect line along the flow direction. Both ends are detached and displaced, leaving the disclination length constant. This is indicated by the two black stripes which remain attached to the displaced core. On turning the flow off, the disclination relaxes back to its initial position.

depins only from one surface, the disclination line elongates under the flow, as demonstrated by the image sequence in Fig. 6.10, In the first and last images of the Fig. 6.10a, there are four dark stripes around the $(-1)$ disclination line, while in the intermediate images, there are four dark stripes around each end of the line. This indicates that the line stretches while pinned only on one surface. In the other possible outcome, both ends of the disclination depin from the channel surface. This scenario is depicted by the time sequence for a $+1 / 2$ disclination line in Fig. 6.10 p. In contrast to the previous situation, here the two dark stripes are observed throughout the sequence, indicating that the line detached from both ends and gets dragged by the flow. The local pinning strength on the confining surface decides whether one or both ends of the disclination will be detached. The disclinations relaxed back to their original position when the flow was stopped. In the absence of any relative elongation (Fig. 6.10b), surface memory effects help in reinstating the disclination line after the flow is stopped. On the other hand, for disclinations which underwent elongation (Fig. 6.10 $)$, there is an additional contribution from the free energy minimization, owing to the reduction of the disclination length. This is qualitatively evident from time scales shown in the figures $6.10 \mathrm{a}$ and $6.10 \mathrm{p}$. Moreover, such deformations are dynamically preserved in the presence of the flow. 


\section{Surface-stabilized disclinations}

On increasing the Ericksen number $(900<E r<1250)$, the disclination line at the leading end of the $\pi$-wall depins generally from the glass surface and aligns itself horizontally in the flow direction. The depinned disclination, stretched up to hundreds of micrometers by the flow field, repins downstream on the same PDMS surface of the microchannel, thereby reorienting itself (Fig. 6.11). Such horizontally aligned disclinations emanate from the leading end of both types of $\pi$-walls discussed in Section 6.3 . Textural similarity and high morphological stability (with/without flow) suggest that the defect lines from both types of $\pi$-walls are stabilized singular lines, without any direct evidence of director field escape into third dimension. Figure 6.11a shows an unpolarized micrograph of such an integer strength disclination line arising from the end of the $\pi$-wall. In contrast to the $\pi$-wall structure, which can be distinguished only between crossed polarizers (Fig. 6.11b), the surface-stabilized disclinations are clearly visible also in unpolarized light. The overall nematic texture in the $x y$ plane is schematically illustrated in Fig. 6.11k. Detailed information about the spatial molecular distribution is obtained from the FCPM imaging. The confocal $x y$ scan of the $\pi$-wall texture in Fig. 6.11d matches with the one in Fig. 6.8 $\mathrm{d}$. However, the presence of the additional intensity minimum initiating at the leading end of the $\pi$-wall corresponds to the bright texture in Fig. 6.11 b. More detailed information, extracted from $y z$ cross-sections in Fig. 6.11 d, clarify that the director field near the leading end of the $\pi$-wall undergoes a gradual splay-bend deformation. From the polarizing fluorescence intensities, one can conclude that such disclinations stabilize close to the PDMS surface. The resulting director orientation is proposed in Fig. $6.11 \mathrm{~d}$.

The above described creation and behaviour of $\pi$-walls in microfluidic environment appears to be a prominent feature in the case of degenerate planar surface anchoring on the channel walls. Although the generation of the defects in this case is a random process, the structures offer interesting templates in regards to our discussions in Section 6.2. The set of $\pi$-walls represent a spatial variation of the director field at regular intervals. Hence, the influence of the flow-director coupling is expected to leave its imprint on the velocity profile through such a structure. Subsequent experiments have revealed that the development of such diverse textures is generally absent in similar flow and confinement conditions with homogeneous (planar or homeotropic) surface anchoring. However, at very high Ericksen numbers, the chaotic regime can be attained under various anchoring situations (Section 6.7). 

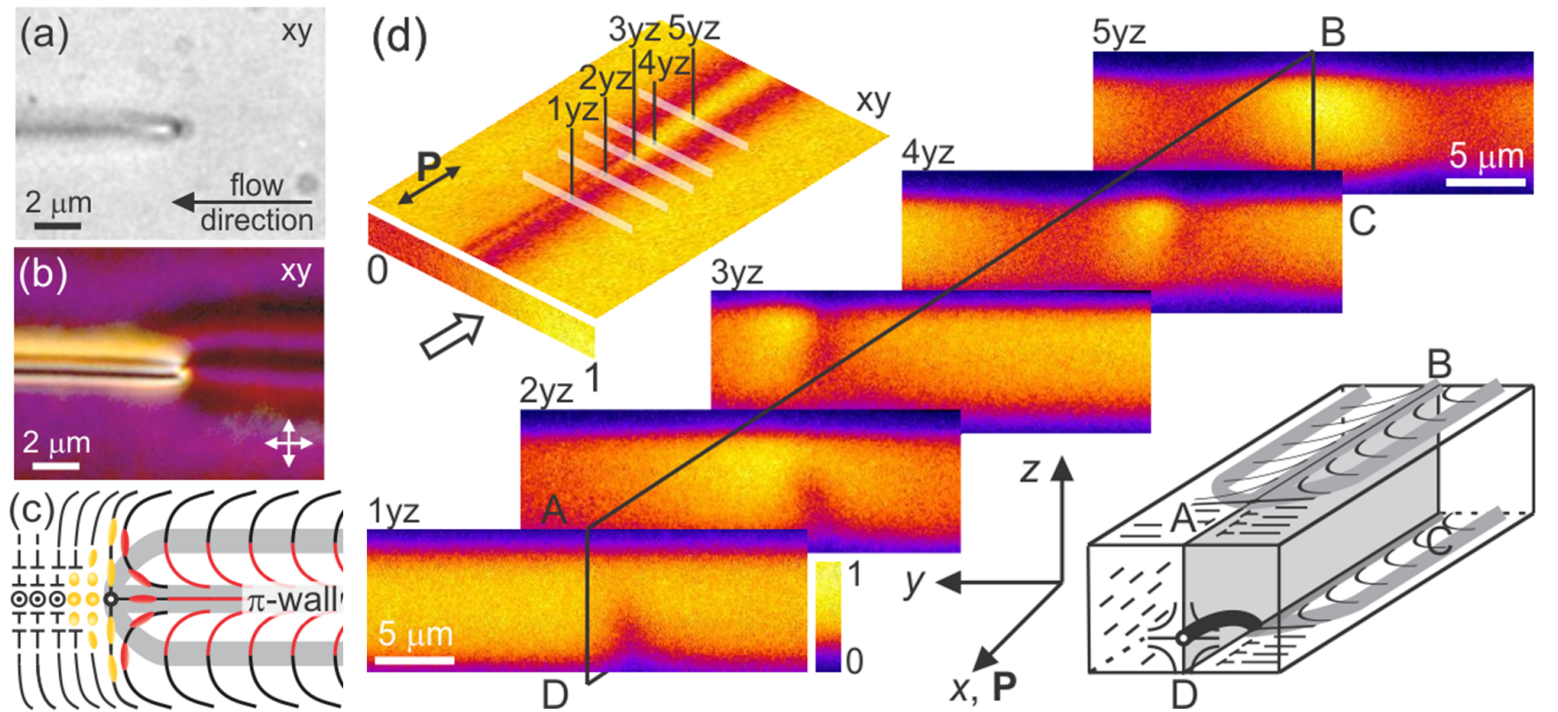

Figure 6.11: Morphology of a surface-stabilized disclination. (a) Unpolarized micrograph. The dark line indicates the light scattering from the disclination line at the leading end of the $\pi$-wall, aligned along the channel. The $\pi$-wall itself is not visible. (b) Polarized optical micrograph shows high contrast disclination line which arises at the end of alternate dark and bright regions corresponding to the $\pi$-wall. (c) Schematic representation of the director profile in $x y$ plane. The red curves indicate the continuous $\pi$-wall along the channel length. (d) FCPM micrograph in $x y$ plane. FCPM image series in the $y z$ plane on traversing through the disclination line and subsequently entering the $\pi$-wall, from $1 y z$ plane to $5 y z$ plane.

\subsection{Nematic flow in a homeotropic microchannel}

Microchannels possessing homeotropic surface anchoring provide one of the more straightforward means to study flow-induced director reorientation under controlled boundary conditions. The steps involved to induce homeotropic boundaries are simpler than the ones required for uniform planar or hybrid alignment. Furthermore, when compared to the case of degenerate planar anchoring, the analyses of these experiments are rather direct, based on the direct observations from optical microscopy (Fig. 6.12). Previously, we have discussed the distinct attribute of NLC hydrodynamics: flow-director coupling, and how this can be explored for modulation of fluid flows. In contrast, flow modulations of isotropic fluids is achieved through innovative lithographic architectures or external fields. This is due to the inherent Poiseuille-like flow profiles [8, 201] of isotropic fluids. Explorations beyond this Poiseuilledetermined regime could open new microfluidic phenomena and applications [218, 219]. Although experimental investigations of complex fluids in microfluidic confinements have been 

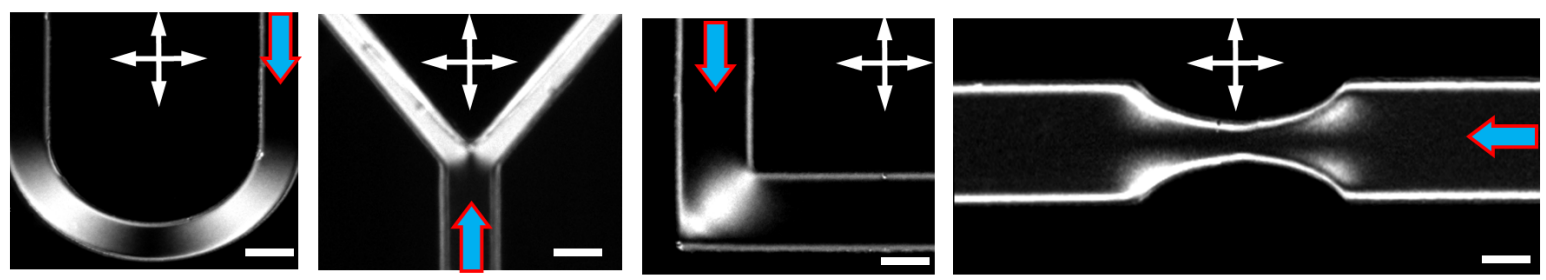

Figure 6.12: Flow alignment in microchannels with homeotropic surfaces. Polarized optical micrographs of nematic $5 \mathrm{CB}$ traversing different curves: $\mathrm{U}, \mathrm{Y}, \mathrm{L}$, and hyperbolic contraction in the flow path. Maximum transmission intensity signifies that the in-plane director is oriented at $\pi / 4$ to the crossed polarizers. Scale bar: $100 \mu \mathrm{m}$.

initiated [31, 38, 39, 220, 221], understanding of their dynamics is mostly limited to numerical studies [222-228]. In particular, investigations on liquid crystals as a natural choice for an anisotropic replacement of the isotropic fluids have been directed towards effects mediated by topological defects [24, 60, 114, 121, 192, 229-231].

Owing to the coupling between the orientational order and the flow, any change in the orientational profile affects the flow, and any change in the flow causes a change in the orientational profile. It is only very recently that experiments on backflow dynamics in microfluidic setups are being carried out: reporting on flow induced transitions [42, 43], effects of molecular pretilt at confining surfaces [44], nematic textures in untreated channels (see (1) on the publication list), and electrically or mechanically modulated microflows [41, 232].

\subsubsection{Tunable flow shaping}

In this section, we shall look into the complex non-Poiseuille multi-stream flow profiles of NLCs in rectangular homeotropic microchannels, offering a direct route to controlled shaping of the flow profile in a microfluidic channel. The non-Poiseuille flow profile can thus be easily and effectively controlled, e. g., by externally applied temperature gradients. Three flow regimes were identified when nematic 5CB flowed through the channels: (i) in the weak flow regime the nematic flow and orientational order were only weakly coupled and were mostly determined by the surface, (ii) in the medium flow regime the largest variability in both flow and nematic profile was observed, caused by the intricate backflow coupling, and (iii) the strong flow regime is determined by flow-alignment of the NLC molecules. The dynamics were characterized by the de Gennes characteristic shear-flow lengths $e_{1}$ and $e_{2}$ [6] which, together with the channel's aspect ratio $w / d$, control the relative stability of the flow regimes. 

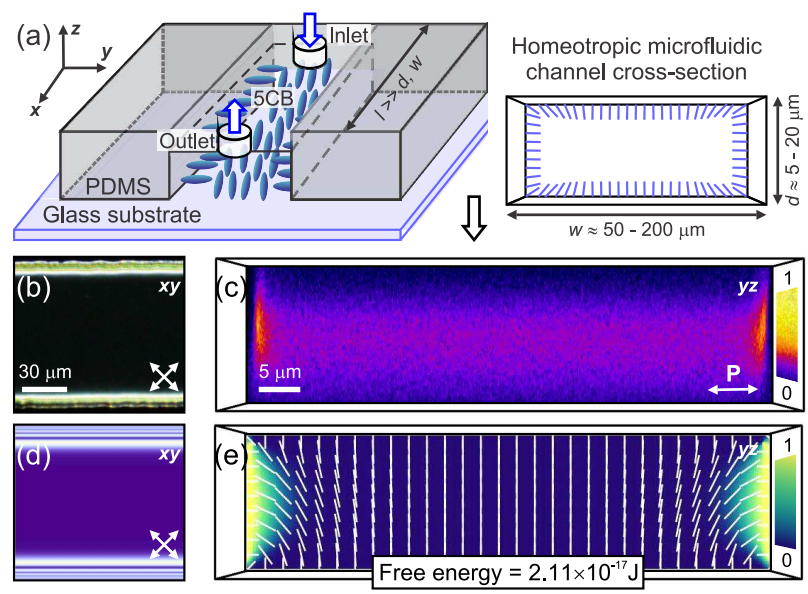

Figure 6.13: No-flow nematic profile in a rectangular homeotropic channel. (a) Scheme of the microfluidic setup. Experimental (b) POM and (c) FCPM micrographs of a $100 \mu \mathrm{m} \times 10 \mu \mathrm{m}$ channel and corresponding numerically modeled (d) POM and (e) FCPM images with director and total free energy. Numerical modeling was carried out by Miha Ravnik.

Finally, by applying local temperature gradients across the channel, the steering of micro-flow streams via mechanisms of nematic anisotropy was realized.

The numerical modeling (carried out by Miha Ravnik) was based on solving the BerisEdwards model of nematofluidics with the hybrid lattice Boltzmann algorithm complements the experiments [223, 224]. Fluorescence confocal signal intensity $I$ was calculated from the local director $n_{i}$ and laser polarization $P_{i}$ as $I \propto\left(n_{i} P_{i}\right)^{4}$, and the POM micrographs were calculated with the Jones $2 \times 2$ matrix formalism [194].

In the absence of flow, homeotropic microchannels support multiple nematic configurations, either stable or metastable, depending on the topological structure of the defects in the corners of the channels (Chapter 5.3). Experimentally, always a single - stable - structure emerged (see Fig 6.13b and c), which indeed has the lowest total free energy, as confirmed by numerical modeling. This equilibrium no-flow structure proves important upon starting the flow, as it registers the initial state for the subsequent dynamics.

Upon starting the flow, three distinct flow regimes are observed, which we categorize into (A) weak, (B) medium, and (C) strong, as presented in Fig. 6.14. In the weak flow regime, the nematic structure remains similar to the no-flow configuration, with the director perpendicular to the flow velocity. The microchannel is dark between crossed polarizers (Fig. 6.14A-a) and gives low FCPM signal (Fig. 6.14A-b) at both $x y$ and $y z$ planes, except close to the walls 


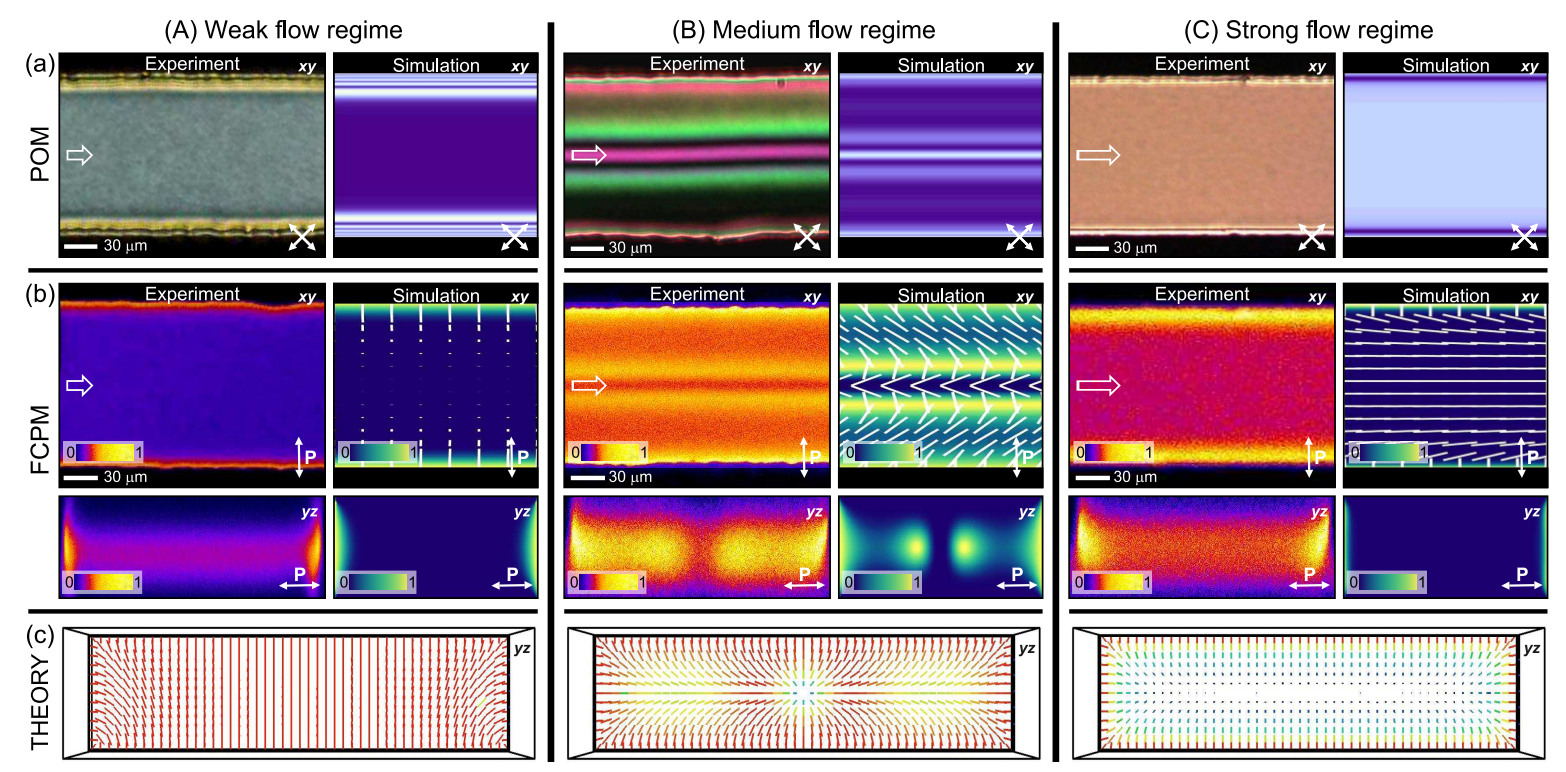

Figure 6.14: Three basic flow regimes in the homeotropic microchannel - (A) weak flow, (B) medium flow, and (C) strong flow. Results show: (a) experimental and calculated POM micrographs in the $x y$ plane, (b) experimental and calculated FCPM micrographs in the $x y$ and $y z$ planes including the director configuration at the half-depth of the channel. 0 and 1 respectively indicate nematic director orthogonal and parallel to the excitation laser polarization. (c) The full director profile calculated across the channel cross-section for the three regimes. Blue colour indicates out-of-plane director orientation, whereas red colour corresponds to inplane director orientation. Experiments were performed in a $16 \mu \mathrm{m}$ deep channel. Numerical modeling was carried out by Miha Ravnik.

due to the conflicting surface anchoring. The corresponding numerical modeling shows good agreement with the experiments, and reveals a distortion of the nematic director field only in the $y z$ plane (Fig. 6.14A-c). Low flow speeds $(\leq 11 \mu \mathrm{m} / \mathrm{s})$ and hence low Ericksen numbers $(E r \approx 1)$ show the dominance of NLC elasticity over the backflow.

In the medium flow regime (flow speeds $v \approx 30 \mu \mathrm{m} / \mathrm{s}$, Fig. 6.14 B), a complex flow rheology evolves, caused by the competition between the nematic elasticity and the backflow. In the centre of the channels, symmetrical changes emerge in the birefringence due to locally high shear rates as imposed by the no-slip at the walls and flow driving in the centres of the channels (Fig. 6.14B-a and b). From numerical simulations, they are found to represent complex bend and splay deformations of the director field, as shown in Fig. 6.14B-c. More specifically, in the central and two symmetric side regions, the backflow realigns the director in the direction along the channel to optimise the coupling between the local shear and the nematic distortions. 


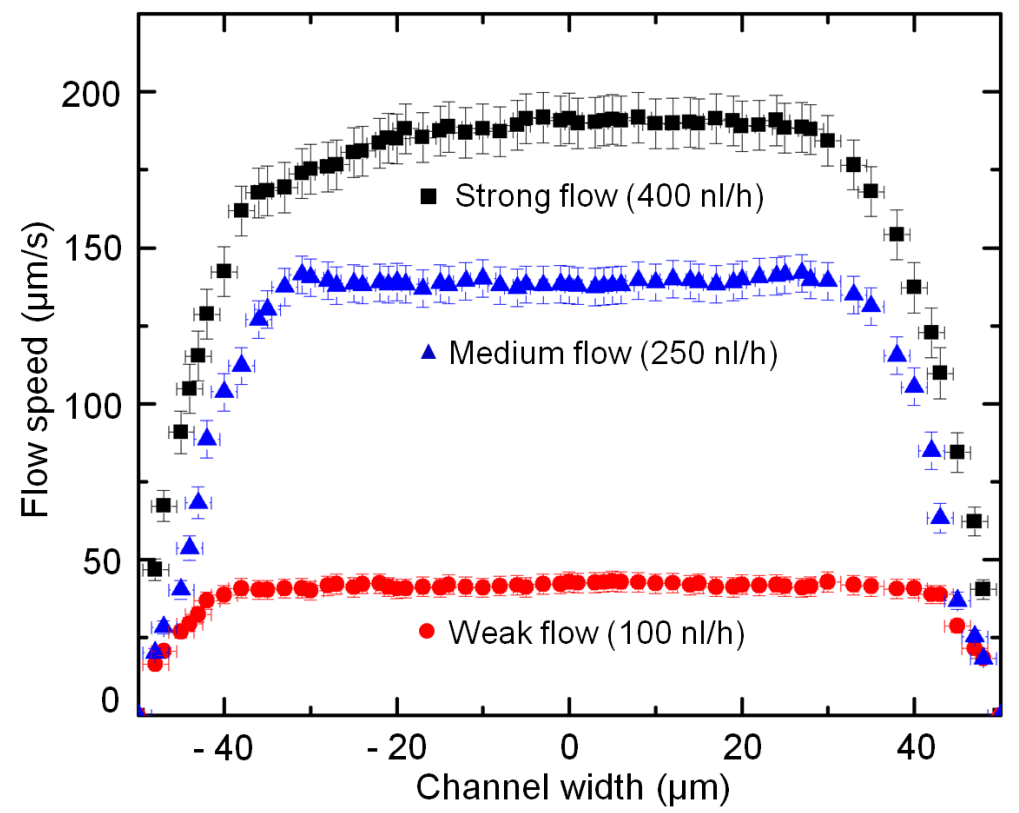

Figure 6.15: Experimental flow profiles at the half-depth of the channel. One stream flow is dominant in the low and high flow regimes. Two stream flow is observed in the medium flow regime.

In the high flow regime (flow speeds $v \approx 100 \mu \mathrm{m} / \mathrm{s}$, Fig. 6.14C), the nematic profile evolves into a flow-aligned state, with the director primarily along the channel direction (Fig. 6.14C-a), as resolved by confocal imaging (Fig. 6.14C-b) and in good qualitative agreement with the numerical calculations shown in Fig. 6.14C-c. Interestingly, this observed highflow-regime nematic profile is analogous to the escaped radial nematic profile, typically observed in nematic-filled capillaries. However, in the present case the bend angle depends on the flow profile.

The corresponding flow profiles of the three regimes are presented in Fig. 6.15 and 6.16, determined by the particle tracking method and numerical modeling. In weak flows, due to weak backflow coupling, the nematic flow profile is similar to those of isotropic fluids, which in rectangular channels can be expressed as a Fourier sum of sin functions along $z$ direction and appropriately weighted cosh functions along the $y$ direction [201]. However, in the medium flow regime, the backflow mechanism emerges significantly. The flow profile evolves into a two-stream flow, identified by a lower velocity region at the channel centre which is surrounded symmetrically by higher velocity regions. Experimentally, the maximum speed in a $7 \mu \mathrm{m}$ deep channel is measured to be $6 \%$ higher than the velocity at the channel 


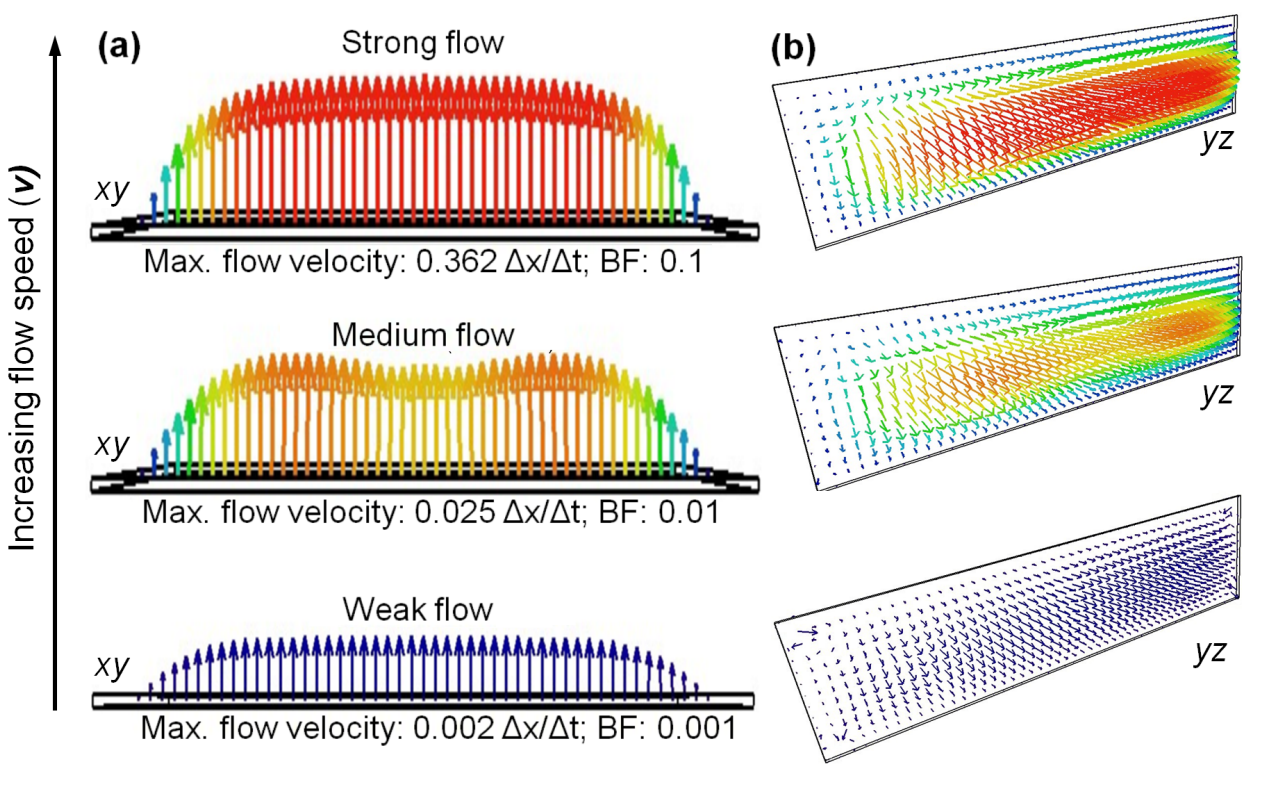

Figure 6.16: Flow profiles of the weak, medium, and strong flow regimes calculated numerically. (a) Flow profiles at half-depth for the three regimes obtained from numerical modeling and (b)the same flow profiles across the full channel cross-section. The body force (BF) quantifies the flow driving force, implemented according to Guo et al. [233]. Numerical modeling by Miha Ravnik.

center. Within deeper channels this difference is more pronounced: Numerical simulations for an aspect ratio of 4:1 yield a speed difference of $12 \%$ (Fig. 6.16). On overlaying the profile to the corresponding director field (Fig. 6.14 B), the lower velocity maps to the splaybend deformation region, whereas the higher velocity regions correspond to regions with the director partially aligned along the flow. Effectively, it is the spatial variation of the apparent viscosity from the backflow mechanism which is responsible for this two-stream flow profile. At strong flows, the flow profile becomes similar to that of isotropic fluids, because the director field is homogeneous except close to the surfaces.

The regimes discussed are stable over a range of flow speeds which depend on the channel depth $d$ (Fig. 6.17). For example, in a $7 \mu \mathrm{m}$ shallow channel, the weak regime persists up to flow speeds of $\approx 40 \mu \mathrm{m} / \mathrm{s}$, whereas in a $35 \mu \mathrm{m}$ deep channel the regime destabilizes at a considerably lower speed of $\approx 10 \mu \mathrm{m} / \mathrm{s}$. Moreover, a flow speed of $\approx 120 \mu \mathrm{m} / \mathrm{s}$ in the deeper channel is sufficient for the director to align parallel with the the flow, whereas only partial alignment is observed in the shallow channel, even at flow speeds $>200 \mu \mathrm{m} / \mathrm{s}$. These differences can be captured by the de Gennes shear flow characteristic lengths $e_{1} \propto \sqrt{K d / 2 \eta v}$ 


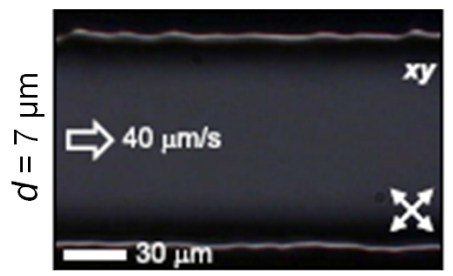

$e_{1}=2.1 \mu \mathrm{m}, e_{2}=3.1 \mu \mathrm{m}$
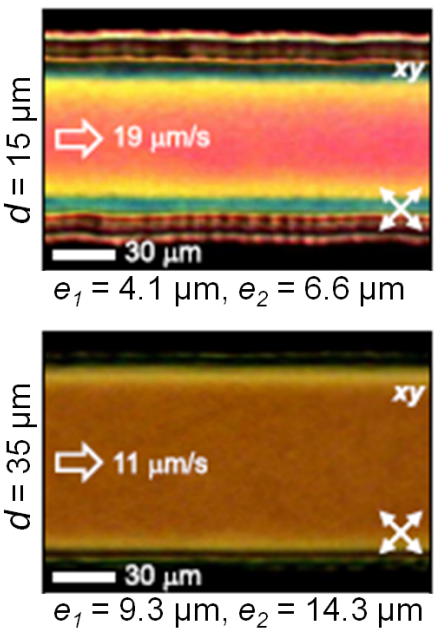

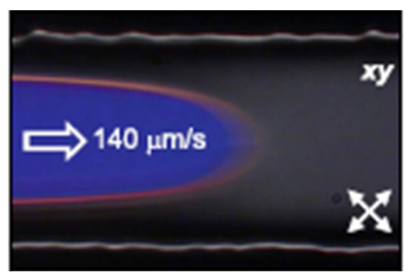

$e_{1}=1.1 \mu \mathrm{m}, e_{2}=2.1 \mu \mathrm{m}$

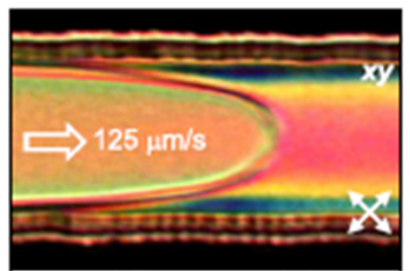

$e_{1}=1.7 \mu \mathrm{m}, e_{2}=3.6 \mu \mathrm{m}$

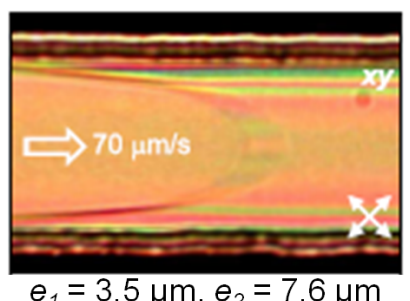

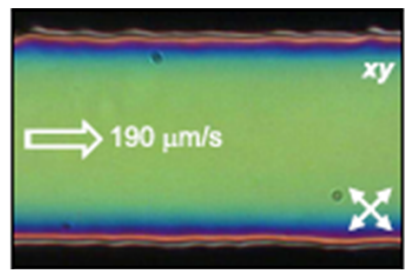

$e_{1}=0.9 \mu \mathrm{m}, e_{2}=1.8 \mu \mathrm{m}$

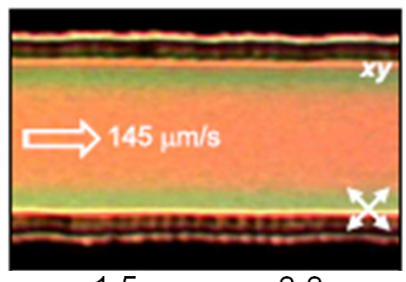

$e_{1}=1.5 \mu \mathrm{m}, e_{2}=3.3 \mu \mathrm{m}$

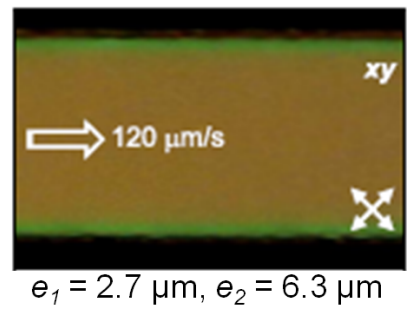

Figure 6.17: POM micrographs representing the evolution of the flow regimes in channels with three different depths. Note the appearance of the parabolic front upon increasing the flow speed. $e_{1}$ and $e_{2}$ values are calculated from experimentally measured average shear rates.

and $e_{2} \propto \sqrt[3]{d e_{1}^{2}}[6]$, expressed in terms of the average elastic constant $(K)$, and the material viscosity $(\eta)$. The characteristic lengths measure the widths of the transition layers - near the walls $\left(e_{1}\right)$ and at the centre of the channel $\left(e_{2}\right)$ - over which the director is reoriented by the local shear flow. For a given channel depth, as the flow speed increases, $e_{1}$ and $e_{2}$ decrease. $2 e_{1}+e_{2} \sim d$ corresponds to the cross-over point, and the transition to the flow aligned state occurs when the flow speed is increased further. Thereafter, one has only the transition layers at the channel walls, whereas at the centre the flow is aligned. Using the estimate $K /(2 \eta)=25 \times 10^{-12} \mathrm{~m}^{2} \mathrm{~s}^{-1}$, in the shallow channel (Fig. 6.17, $\left.d=7 \mu \mathrm{m}\right), e_{1}=2.1 \mu \mathrm{m}$ and $e_{2}=3.1 \mu \mathrm{m}$ are obtained from the measured average shear rate $s=v / 0.5 d$ and maximum flow speed $v=40 \mu \mathrm{m} / \mathrm{s}$. These sum up to the full depth of the channel, $2 e_{1}+e_{2} \sim d$, and mark the transition. In contrast, in the deep channel (Fig. 6.17, $d=35 \mu \mathrm{m}$ ), a similar condition is achieved only at $v \leq 11 \mu \mathrm{m} / \mathrm{s}$. 


\subsubsection{Application of a transverse temperature gradient}

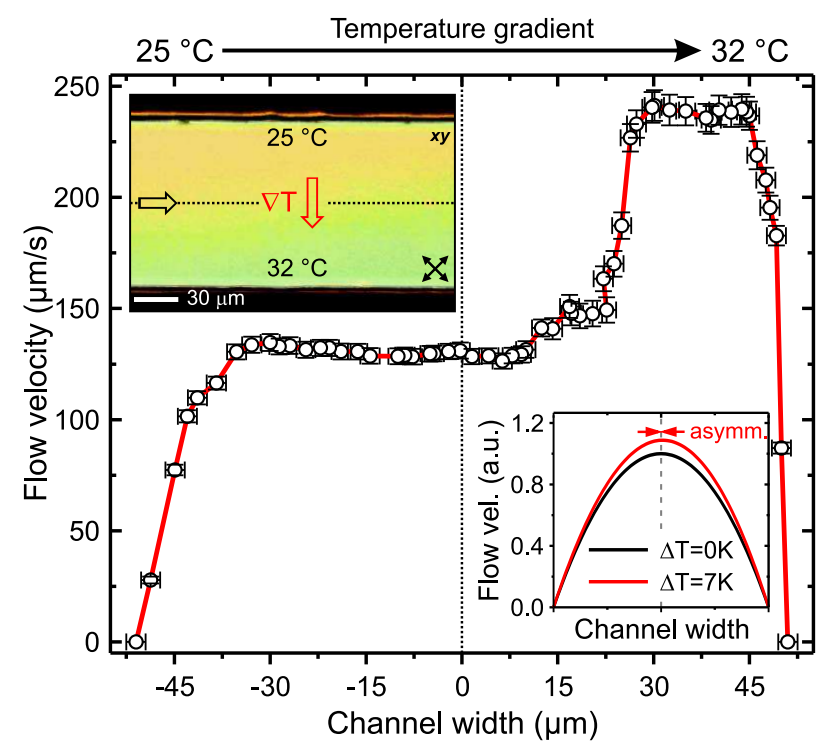

Figure 6.18: Steering of the micro-flow profile by a transverse temperature gradient. The opposite transverse channel walls are temperature stabilized at $25{ }^{\circ} \mathrm{C}$ and $32{ }^{\circ} \mathrm{C}$. The topleft inset shows a top view polarization micrograph of the channel. Channel depth is $10 \mu \mathrm{m}$. For comparison, the bottom-right inset shows the typical change of the Poiseuille flow in an isotropic fluid upon applying an equal temperature gradient, causing changes in the viscosity of $\alpha_{\eta}=\Delta \eta / \eta=-0.175$ [201].

Additionally, the temperature-induced flow modulation can be superposed to that induced by the confinements. A transverse temperature gradient, applied across the microfluidic channel, was used to controllably steer the flow profile. The temperature gradient was set up with the help of a DC voltage applied at the ends of a heating element (wire) placed parallel to one of the side walls of the channel. The other side of the microchannel was left at room temperature. The temperature was controlled by varying the voltage applied across the wire. In isotropic fluids (e. g. water or oil), applying a temperature gradient is a standard way to introduce an asymmetry in the flow profile by affecting primarily the viscosity of the fluid [201]. However, for such fluids, this effect is typically small, and changes mostly the magnitude and only weakly the profile of the flow (see inset in Fig. 6.18). However, in the nematic fluid, the temperature gradient leads to a gradient in the nematic ordering which in turn affects the backflow, and induces a strong bias in the flow profile, as shown in Figure 6.18. A distinct flow stream emerges at the higher temperature side of the channel, which is by a factor of $\sim 2$ 
larger than the flow velocity on the (symmetric) opposite side of the channel. Furthermore, the position and magnitude of this flow stream can be tuned by varying the temperature gradient. A similar flow steering can also be achieved in the medium and weak flow regimes, and indicates interesting possible applications for sorting and colloidal manipulation based on nemato-microfluidics.

The backflow mechanism in nematic fluids thus proves to be a strong route to sensitively tuned complex micro-flows. Three flow regimes -weak, medium, and strong- were observed in rectangular microchannels with uniform homeotropic boundary conditions, each characterized by distinct director and flow profiles. A multi-peak flow distribution is achieved in the channels without any morphological patterning or advanced channel designing. Asymmetric flow streams were generated and precisely steered by transverse temperature gradients, indicating high potential for diverse flow-shaping. The dynamically regulated nematic flow profiles observed indicate interesting applications beyond the capabilities of isotropic microfluidics, including flow mixing, large scale transport, high throughput separation of colloidal inclusions, and active-response optical detection of pressure changes within microfluidic networks.

\subsubsection{Opto-fluidic velocimetry in a diverging channel}

The flow speed within a microchannel can be varied in situ by replacing a linear channel by one whose width varies in the downstream direction. While for a diverging channel the width $w$ increases, for a converging channel the width decreases in the downstream direction. Consequently, the coupling between flow and orientation of nematic liquid crystal molecules could be tuned by employing a microchannel with variable width. In this section, we shall see how a diverging geometry has been utilized to devise a non-intrusive opto-fluidic velocimetry technique on a microfluidic platform (Fig. 6.19a). The flow-induced reorientation of the liquid crystal molecules in a diverging channel possessing homeotropic surface anchoring produced distinct birefringent domains, directly observable through their interference colours, which are characteristic to the local flow velocity. The flow-induced effective birefringence was characterized using polarizing optical microscopy, confocal fluorescence polarizing microscopy, and particle tracking methods. Essentially, the coupling between flow and director orientation was optically assessed, which was then exploited to determine the velocity profiles of the flowing NLC.

Consider a NLC on a plane substrate with homeotropic anchoring condition and let $z$ denote the direction perpendicular to the substrate (Fig. 6.19 b). When a shear flow parallel to the 


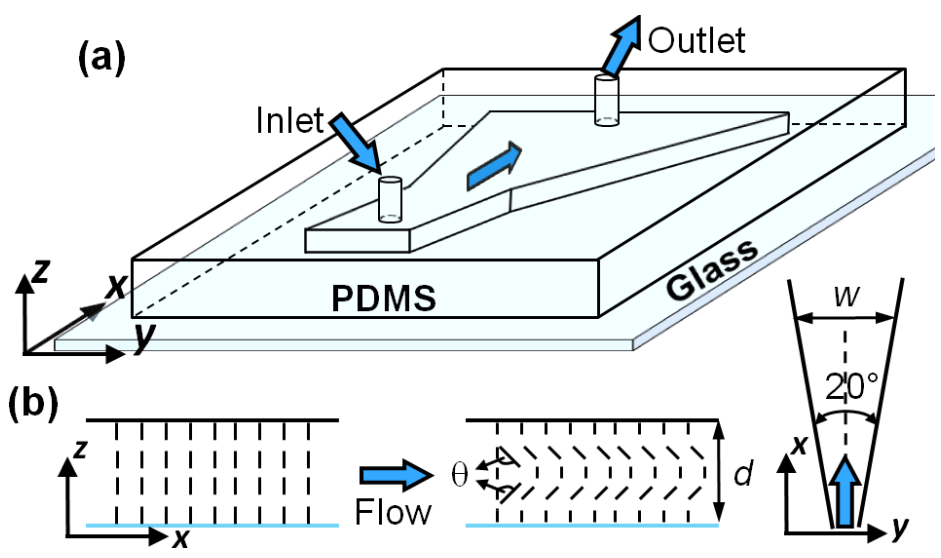

Figure 6.19: Schematic diagram of (a) the PDMS-Glass diverging microchannel and (b) the homeotropic (normal) anchoring of the NLC molecules on the channel surfaces. In presence of flow, the molecules reorient by an angle $\theta$. Plan view of the microfluidic channel indicating the diverging angle and the flow direction.

substrate is initiated, the initial equilibrium director field (in which $\vec{n} \| z$ everywhere) experiences an elastic deformation: Due to the viscous torque induced by the flow, the local director rotates in the direction of flow by an angle $\theta$, relative to the initial orientation. In the present system, with increasing strength of the shear flow, $\theta$ increases until a saturation value

$$
\theta_{\text {sat }}=\pi / 2-\tan ^{-1} \sqrt{\alpha_{3} / \alpha_{2}}
$$

is reached at which the viscous torque on the director vanishes. Eq. 6.2, which is derived from the continuum model proposed by Leslie and Ericksen [12, 14, 15], is applicable for positive values of the ratio of the two Leslie viscosity coefficients $\alpha_{2}$ and $\alpha_{3}$ [16]. For the Poiseuille flow in a microchannel with a small aspect ratio, we can approximate the configuration by considering it as being composed of two halves possessing shear-like flow profiles, leading to a deformation of the director field as sketched in Fig. 6.19p.

The perturbation of the director field can be visually observed in white light microscopy by placing the sample between crossed polarizers. Because of the homeotropic anchoring conditions, the sample exhibits at zero flow apparently no birefringence along the $z$ direction. With increasing flow velocity $v$ (and thus increasing $\theta$ ), the effective birefringence along $z$ increases and, for an appropriate film thickness, one can expect a sequence of interference colours to appear. In a diverging microchannel, a gradient of flow speed exists long the channel length at a constant volumetric flow rate $r_{V}$. For a given value of $r_{V}$, the nematic director 

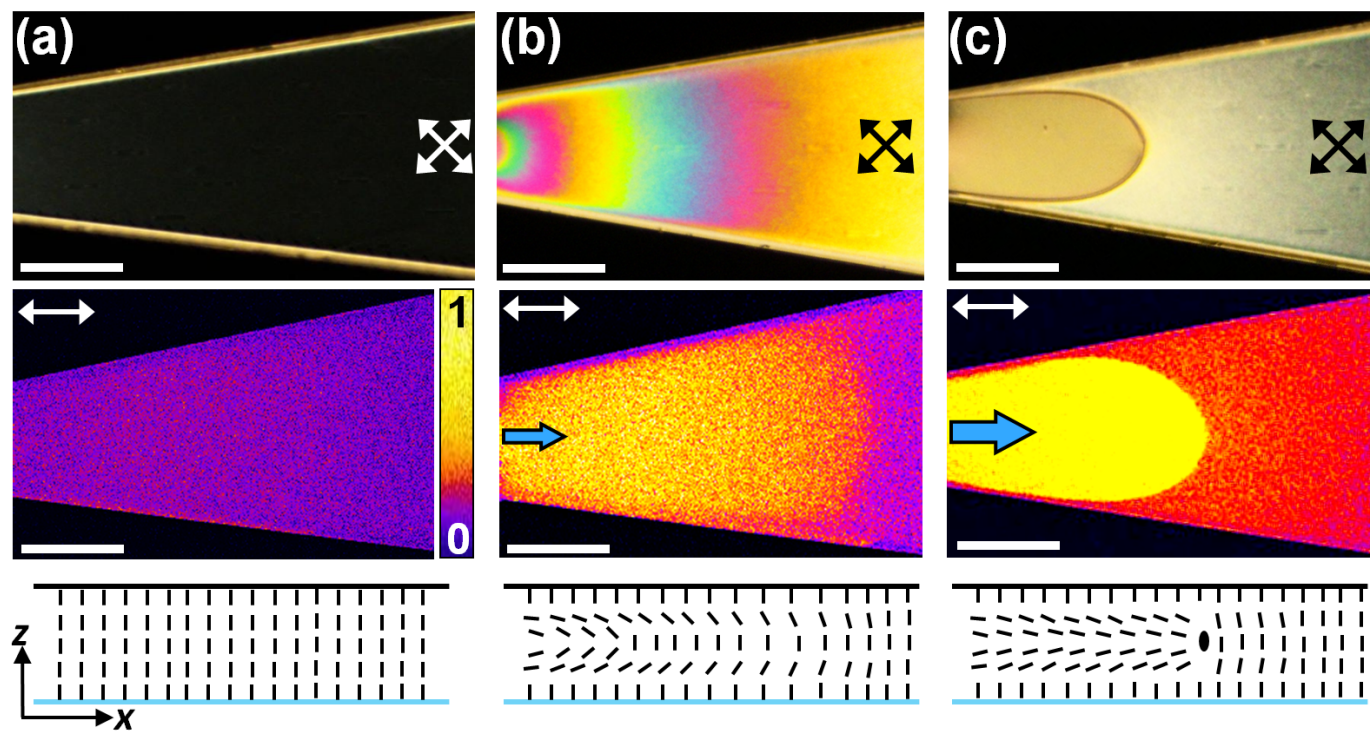

Figure 6.20: Perturbation of the nematic director. (a) POM (top row) and FCPM (middle row) micrographs of a channel filled with 5CB in absence of any flow. (b) At low flow rates, colourful birefringent domains appeared, and corresponding FCPM micrograph with laser polarized along the flow direction. (c) Snapshot during a rapidly increasing flow rate. Two distinct domains are observed. High FCPM intensity indicates strong director reorientation by the flow, compared to the weak distortion in the channel downstream. The domains are separated by a defect structure which moves downstream. The bottom row schematically represents the molecular orientation in each case. Scale bar: $300 \mu \mathrm{m}$. See also Fig. 6.21.

attains different orientations at different regions of the channel, according to the different local values of $v$. Since $v$ decreases from the inlet towards the channel outlet, so does the distortion of the nematic director, resulting in a static sequence of interference colours resembling a nonlinearly stretched Michel-Lévy chart (Chapter 3.4.1). With a single calibration, using the particle tracking method, one can assign to each colour a value of $v$. The relation between the interference colour and $v$ depends, for a given NLC, only on the channel depth, thus enabling the non-intrusive determination of flow velocity profiles in arbitrary channels possessing the same depth.

It might be practical to briefly revisit the concept of birefringence, discussed previously in Chapter 2.5.1. Due to the optical anisotropy inherent in NLCs, the index of refraction parallel to the director, $n_{\|}$, is different from that perpendicular to it, $n_{\perp}$. When light intercepts the optical axis at an angle $\phi$, it splits into ordinary and extraordinary components, travelling at different speeds determined by effective refractive indices $n_{e}$ and $n_{o}$. The phases of the two 


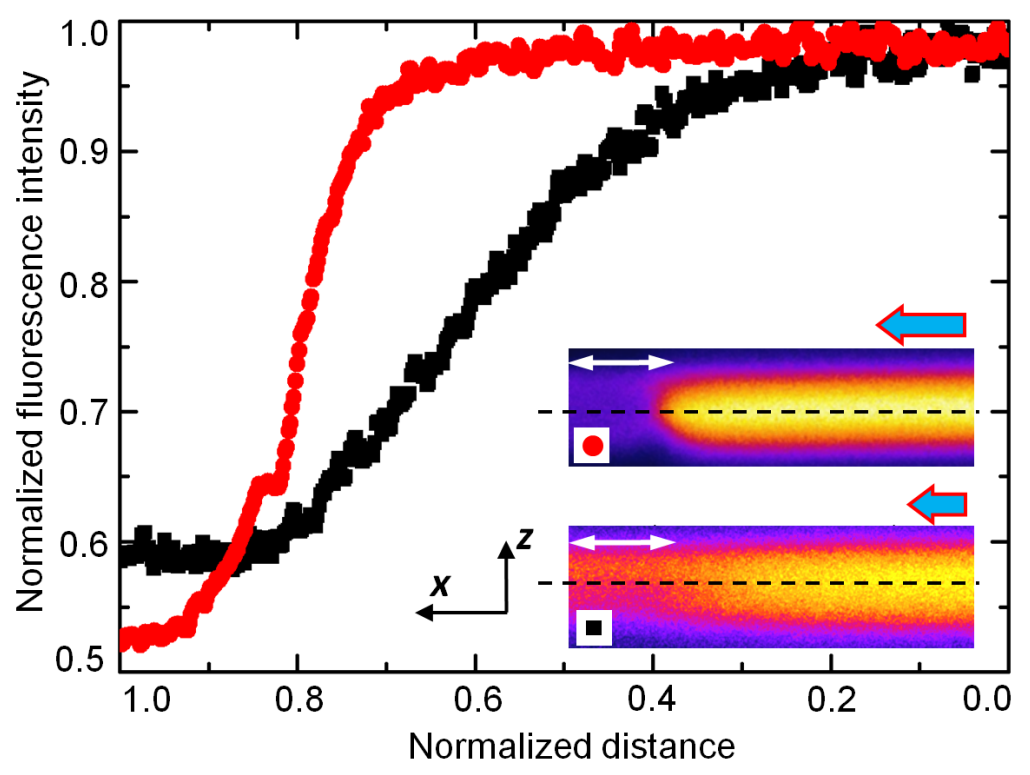

Figure 6.21: Fluorescence intensity distribution about $x z$ mid-plane corresponding to Fig. $6.20 \mathrm{~b}$ and c. Note the steep increase of the fluorescence signal in the rapid flow (red), when compared to the gradual increase at low flow rates (black). The intensities in each case was normalized by the corresponding maximum intensity.

components are equal if

$$
m \lambda=\left(n_{e}-n_{o}\right) d
$$

where

$$
\begin{aligned}
& n_{e}=\frac{n_{\|} n_{\perp}}{\sqrt{n_{\|}^{2} \cos ^{2} \phi+n_{\perp}^{2} \sin ^{2} \phi}} \\
& n_{o}=n_{\perp}
\end{aligned}
$$

and $d$ is the optical path length. The parameters $\Delta n=n_{e}-n_{o}$ and $m$, which denote the effective birefringence and the corresponding order respectively, are decided by the relative orientation of the optical axis to the direction of light propagation.

Figure 6.20a shows POM and FCPM images of the diverging channel, filled with 5CB, at zero flow between crossed polarizers oriented at $45^{\circ}$ with respect to long channel axis. Because of the homeotropic anchoring on the channel walls, the effective birefringence is zero and the sample appears uniformly dark between crossed polarizers. However, close to the side walls the director field experiences a bend deformation, consequently producing al- 
ternating dark and bright parallel lines due to interference of the ordinary and extraordinary components of the transmitted polarized light. The FCPM micrograph in Fig. 6.20a shows a very weak fluorescence signal within the channel, thus confirming the homeotropic alignment. On the contrary, when flow is initiated, a colourful pattern is produced (Fig. 6.20p) in the vicinity of the channel inlet. On gently increasing the flow rate, the pattern travels downstream, eventually occupying the entire length of the channel. Each colour visualized by POM corresponds to a specific angle of interception between the polarized light and the mean orientation of $\vec{n}$. Analogously, the colours signify the extent of the flow mediated reorientation, $\theta$ (Fig. 6.19b), undergone by the director field. The gradual decay of the FCPM signal from the inlet towards the channel outlet further reveals the continuous decrease of $\theta$ in the downstream direction. Figure 6.21 shows the variation of the fluorescence intensity measured along the $x z$ cross-section, with the laser polarization parallel to the flow direction. The intensity distribution measured across the vertical mid-plane passing through the channel (data points black in colour), clearly show the gradual decay of the signal along the downstream direction. In contrast to the high intensity at the inlet (stronger reorientation by flow), the weak intensity downstream suggests smaller reorientation. The appearance of the colours in the polarized micrograph (Fig. 6.20 p), attributed to the director distortion, thus varies with the flow velocity along the channel length. The position of a particular hue (e. g., magenta) is stationary for a given volume flow rate $r_{V}$. However, as $r_{V}$ is increased, the first 'magenta' domain shifts downstream and gives way to the second one through a range of intermediate colours. Evolution of these successive orders ceases with the emergence of a uniform birefringence at higher flow velocities, signifying the attainment of the limiting reorientation angle $\theta_{\text {sat }}$. Fig. 6.20 shows a non-equilibrium situation observed during a rapid increase of $r_{V}$ : In the region around the inlet, the director instantaneously achieves flow-alignment due to the high flow speed, as indicated by the bright FCPM signal ( $\approx 1$ on the intensity scale). Further downstream, the director field is still relatively undisturbed owing to lower velocities, and a contrasting domain of weak distortion is observed. This is confirmed by the FCPM measurements along the vertical mid-plane passing through the channel. In contrast to the previous case, the decay of the fluorescence intensity is steep, as shown in Fig. 6.21 (set of red data points). The two domains are separated by a meta-stable singular defect structure which moves downstream. When $r_{V}$ remains at a large constant value, the flow-aligned region occupies the complete channel within a short time. In the following, let us focus on the case of medium flow rates by which gradual director reorientations are obtained (Fig. 6.20 b).

Figure 6.22 shows POM micrographs demonstrating the development of birefringent pat- 


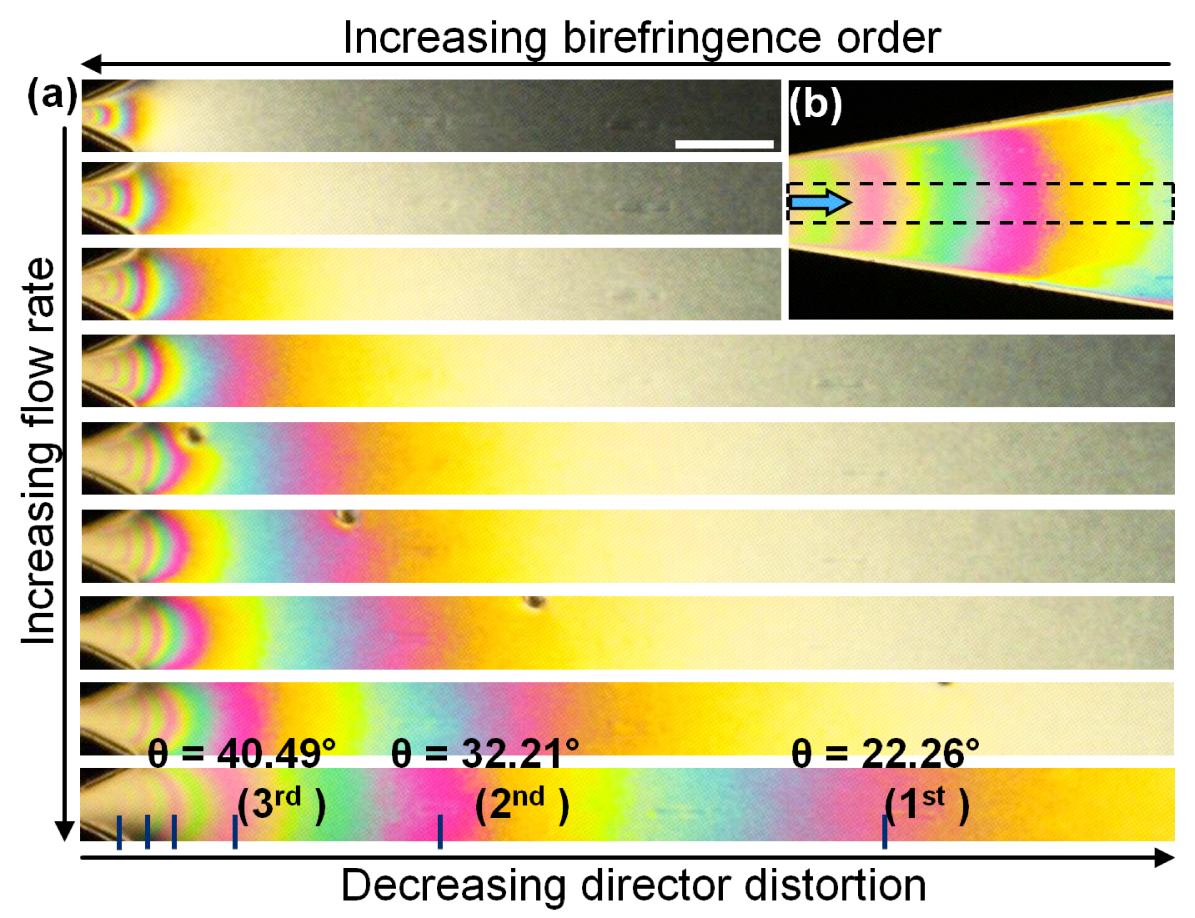

Figure 6.22: (a) POM micrographs showing the evolution of the birefringent domains with gradual increase of the volume flow rate as observed within the region marked in the inset (b).

terns with increasing flow rate (top to bottom) in a $50 \mu \mathrm{m}$ deep channel. At very small flow rates $\left(r_{V}<100 \mathrm{nl} / \mathrm{h}\right)$, no significant birefringence is observed, except near the inlet. When $r_{V}$ is increased above $100 \mathrm{nl} / \mathrm{h}$, the first order magenta appears at a local flow velocity $v \approx 10$ $\mu \mathrm{m} / \mathrm{s}$ (as determined from the speed of tracer particles). The first order magenta indicates an optical path difference $\Delta d=550 \mathrm{~nm}$. Using Eqs. 6.3,6.5 and the refractive index values of 5CB $\left(n_{\|}=1.683\right.$ and $\left.n_{\perp}=1.516\right)$, we calculate a mean director distortion of $\theta=22^{\circ}$. On increasing $r_{V}$ to $\approx 180 \mathrm{nl} / \mathrm{h}$, the first order magenta proceeds downstream and occupies again the region where now $v \approx 10 \mu \mathrm{m} / \mathrm{s}$. The erstwhile position of the first order is now occupied by the second order $\left(\Delta d=1100 \mathrm{~nm}, \theta=32^{\circ}\right)$ and corresponds to a local velocity $v \approx 16 \mu \mathrm{m} / \mathrm{s}$. On further increasing $r_{V}$, higher orders of interference colours continue to appear: Within a 50 $\mu \mathrm{m}$ deep channel, up to seven orders are perceptibly observed. The total number of observed orders is found to depend qualitatively on the channel depth. Although in deeper channels even higher orders could be achieved, the birefringence contrast weakens significantly beyond the fifth order.

In addition to the position of each order within the channel, the spatial extent of each order 


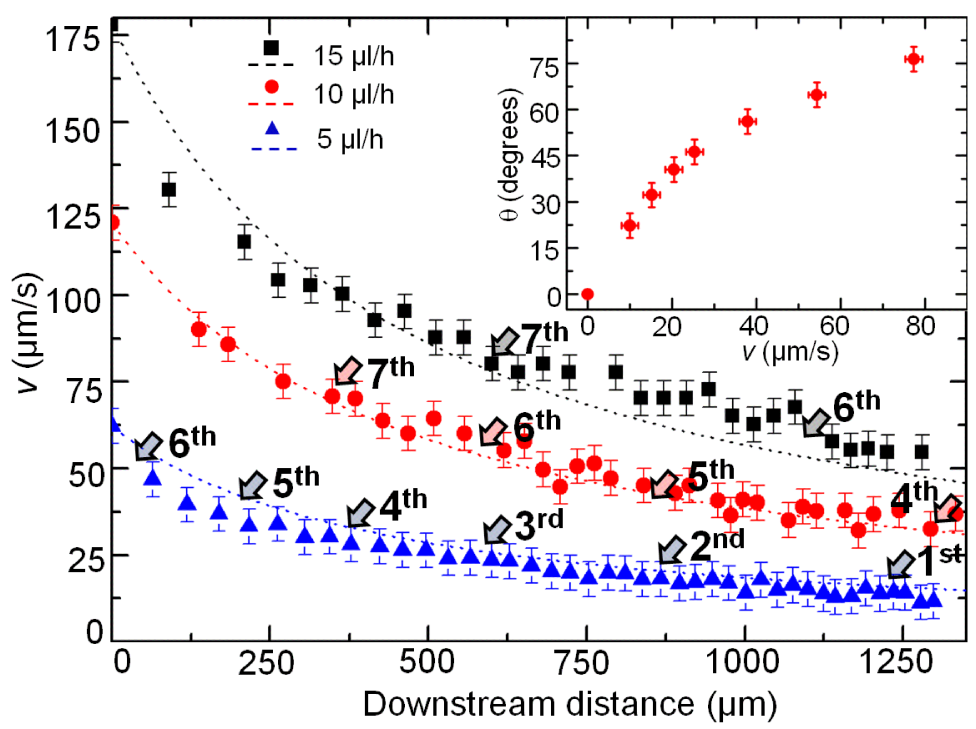

Figure 6.23: Experimental (solid points) and calculated (dotted lines) values of the local flow velocity $v$ as a function of the distance from the inlet along the channel centre for three different volume flow rates in a $50 \mu \mathrm{m}$ deep channel. The position of the different orders of the interference colours are marked on the plots. The inset shows the relation between the distortion angle $\theta$, determined from the interference colours, and the local flow velocity $v$.

is observed to vary along the channel length. As is clearly demonstrated in the micrographs at the bottom of Fig. 6.22, the width of the third order magenta is smaller than that of the second order. The gradient of flow speed in the narrower portion of the channel is higher compared to the wider region. Hence, the local flow velocity and, consequently, the director reorientation decreases more rapidly close to the inlet, in contrast to the wider portions. This variation of the gradient of flow speed was confirmed by the velocity measurement experiments (Fig. 6.23). The width of the birefringent domains could be however tuned by varying the diverging angle of the channel.

Figure 6.23 shows the variation of the flow velocity $v$, determined with the help of tracer particles, along the channel length for three different volume flow rates $r_{V}$ in a $50 \mu \mathrm{m}$ deep channel. On each dataset, the positions of the different orders of interference colours are marked. For $r_{V}=5 \mu \mathrm{l} / \mathrm{h}$, up to six orders of interference colours are observed within the channel. Each order characterizes a distinct value of $v$ and appears reproducibly at a consistent position within the channel. The inset in Fig. 6.23 shows the relation between $v$ and the mean distortion angle $\theta$, determined from the interference colour. With increasing $v, \theta$ increases first linearly with $v$ and approaches then an asymptotic behaviour. From viscosity measurements of 
5CB [138, 139], one expects a saturation angle $\theta_{\text {sat }} \approx 78^{\circ}$ (Eq. 6.2) which is in good agreement with the data presented in the inset of Fig. 6.23. On increasing $r_{V}$ to $10 \mu \mathrm{l} / \mathrm{h}$, the interference colour pattern proceeds downstream. Close to the channel inlet, the director angle attains the limiting value $\theta_{\text {sat }}$ and only four orders $\left(4^{\text {th }}-7^{\text {th }}\right)$ are observed within the same region of interest. At a flow rate $r_{V}=15 \mu \mathrm{l} / \mathrm{h}$, only two orders are observed. The velocity measurements for each flow rate nevertheless reveal the occurrence of a given order at a specific flow speed, with an accuracy of about $10 \%$.

Hence, the relation between the flow velocity and the flow-induced effective birefringence was characterized for the nematic $5 \mathrm{CB}$. By taking into account the appropriate material constants (refractive indices and viscosity), this method can be seamlessly extended to other nematic liquid crystal materials flowing within a diverging microchannel possessing homeotropic surface anchoring. Although the experiment could have been carried out in linear microchannels by varying the pump flow rate, the use of diverging channels produced in situ a range of flow velocities at a fixed volume flow rate, and thus helped to bypass flow-related uncertainties arising from the pump. For a given volume flow rate, multiple orders of interference colours within the channel can be observed and attributed to the magnitude of the local flow velocity. The operating window of this opto-fluidic velocimetry technique can be additionally tuned by varying the channel geometry (depth and diverging angle of the microchannel) and anchoring strength of the liquid crystal on the channel surfaces. Once the relation between the local flow velocity and the interference colour has been calibrated with the help of tracer particles, the technique can be used to determine not just a single flow velocity, but the full (two-dimensional) velocity field in a single step and in real time. Additional applications of the coupling between the flow and the effective birefringence of liquid crystals in microchannels suggest themselves: For instance, the use as an integrated microfluidic tunable optical retarder or as a microfluidic pressure sensing device is conceivable.

\subsection{Nematic flow past a cylindrical micro-pillar}

The study of fluid flow past obstacles is a standard problem encountered frequently in a diverse set of length and time scales: from enhanced oil recovery through porous macroscopic geological structures to flow around colloidal particles and droplets confined within micro or nano confinements. In this section, the flow of a nematic liquid crystal past a micron-sized cylindrical pillar is investigated. The pillar in consideration, fabricated using 
soft lithography (please refer Chapter 3.3.1 for details), was positioned within a microfluidic confinement of rectangular cross-section. The liquid crystal molecules anchored perpendicularly (homeotropic anchoring) to the surface of the pillar and the channel walls. The flow past the cylindrical obstacle generated topological defect structures whose nature, dimension and morphology varied with the flow velocity and channel dimension. On increasing the flow speed, a sequential evolution of topological defects was observed. To begin with, the semiinteger defect loop around the pillar (see Chapter 5.4) was stretched by the flow, which then transformed to an integer hedgehog defect, and finally equilibrated to an extended defect wall. On stopping the flow, the sequence of appearance of the topological defects reversed its order. Additionally, motivated by the controlled creation of the defects, the current experiments were employed for introducing dual-focus fluorescence correlation spectroscopy as a general velocimetry technique for microfluidics of liquid crystal systems, especially ones accompanying topological defects. The dual-focus fluorescence correlation spectroscopy measurements were carried out in the laboratory of Jörg Enderlein with the help of Christoph Pieper.

In the present experiments, the presence of a cylindrical micro-pillar cast in the microchannel induced distinct director reorientations in the vicinity of the obstacle as compared to that farther away from the micro-pillar. Consequently, a sequence of flow-induced topological defects [26] evolved at the pillar. The nature, dimension, and director morphology of the generated topological defects varied with the flow speed and the channel dimensions. In contrast to the observations reported here, numerical studies previously carried out [234, 235] do not report the evolution of topological defects from the obstacles. However, the effect of a flow on topological defects around colloids have been reported by Araki and Tanaka [236]. More recently, the flow of a nematic liquid crystal (NLC) through a homeotropic porous network has shown reconfigurable deformation of defects around the solid portions of the network [193]. The Stokes drag on spherical particles with accompanying topological defects at low Ericksen numbers Er [76] was investigated by Stark et al [237]. Subsequently, the time evolution of flow-induced orientational profiles of defects around colloids [27], and around drops and bubbles rising through a nematic bulk [28, 29] were investigated. Numerically or otherwise, the flow of a nematogen past a cylindrical obstacle and the effect of a high value of $E r$ on the resulting defect morphology have not been reported yet. In addition to studying the flow-induced topological defects and their transformations, dual focus-fluorescence correlation spectroscopy [163] has been used as a technique for measuring the velocity field within the flowing nematogen. Significantly, this technique is suitable for measuring velocities even in a coexisting system of high (nematogen) and low (topological defects) order phases. Con- 
ventional particle tracking methods are unsuitable for the flow field measurements due to the elastic interactions between the dispersed tracers and the topological defects.

In Chapter 5.4, we have discussed the director equilibrium of nematic 5CB in the vicinity of the micro-pillar (and away from it), in absence of any flow. The surface-induced equilibrium of the director configuration is altered as the flow of the nematogen is initiated within the microchannel. The director undergoes a reorientation along the flow direction [76] which increases non-linearly with $v$. In a Poiseuille flow of a homeotropically aligned sample, two different transition layers of director deformation, $e_{1}$ and $e_{2}$ develop (see Section 6.4.1). Due to the difference of the local shear rates close to the surface and at the channel mid-plane $(z=d / 2)$, the thickness over which the nematic director reorients away from the homeotropic alignment, is also different. Consequently, with increasing flow speed and director alignment, the presence of an obstacle leads to the evolution of different kinds of flow-induced defect structures.

The director orientation within the flowing matrix progressively attains the saturation Leslie angle [15], $\theta_{L}=\tan ^{-1} \sqrt{\alpha_{3} / \alpha_{2}}\left(\approx 22^{\circ}\right.$ for nematic 5CB). Here $\alpha_{2}$ and $\alpha_{3}$ are the Leslie viscosities satisfying the Parodi relation $\alpha_{3} / \alpha_{2}>0$, and $\theta_{L}$ is measured relative to the $x y$ plane [16]. Owing to the small dimensions of the channel, the Reynolds number $R e$ is generally low $\left(\approx 10^{-3}\right)$, ensuring a Stokes regime hydrodynamically. However, it is the competing effect between the viscous and elastic parameters of the LC that determines the flow-induced director orientation. The non-dimensional Ericksen number, $E r=\mu \nu d^{*} / K$, captures the relative influence of viscosity $(\mu)$ and nematic elasticity $(K)$ within the flowing matrix. Here $v$, and $d^{*}$, respectively denote the flow velocity and the relevant length scale of the confinement. Ideally at $E r=1$, the viscous and elastic effects balance each other. However, depending upon the boundary conditions, numerical [17] and experimental [22] investigations have shown that the flow-director equilibria could be achieved at $E r>1$ as well.

For a given combination of channel dimensions and boundary condition, $E r$ depends only on the velocity $v$, and determines the flow-induced director orientation. The presence of an obstacle within the microchannel modifies the flow path, and consequently the director configuration. As summarized in Fig. 6.24, a sequence of topological defect structures, generated due to the flow-director interplay, is observed. In absence of flow (see Chapter 5.4], the free-energy barrier between the non-singular and singular director configurations is easily overcome under normal experimental conditions. Therefore, even at a small flow speed of $v \approx 8 \mu \mathrm{m} / \mathrm{s}$, a singular loop stabilizes at the $x y$ mid-plane (Fig. 6.24 $\mathrm{p}$ ), without any significant modification of the initial equilibrium director configuration (Fig. 6.24a). As the speed 


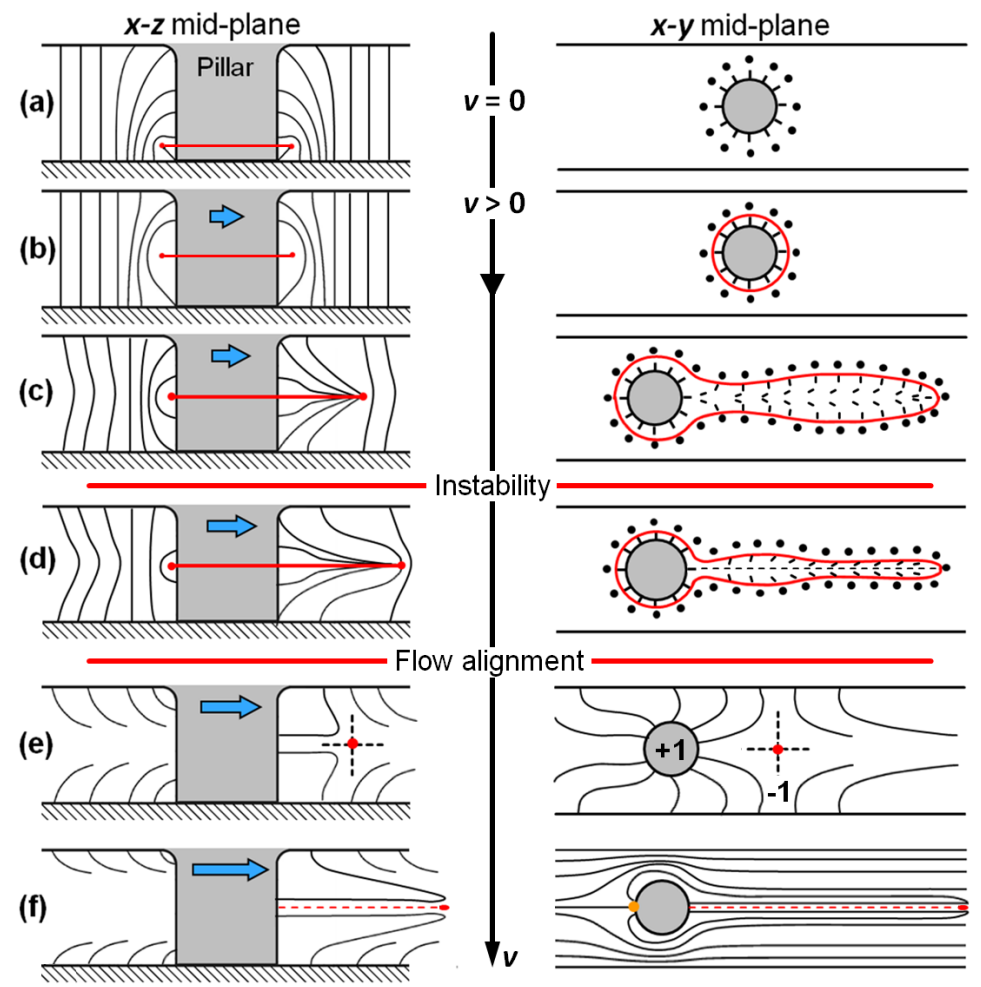

Figure 6.24: Morphological evolution of the defect structures in presence of flow. (a) Semiinteger defect loop around the pillar in absence of flow. (b) Flow or perturbation shifts the loop to the channel mid-plane. (c) At higher speeds, flow-induced director distortion results in extension of the loop along the downstream direction. (d) Instability of the stretched loop sets in just prior to the flow-alignment of the LC director along the $x y$ mid-plane $(z=d / 2)$. (e) The transition to flow-alignment at the mid-plane effects a topological transformation of the loop into a hyperbolic (-1) - radial (+1) hedgehog defect pair. The hyperbolic defect (red dot) downstream to the pillar, and a (virtual) radial defect at the pillar center, form a Néel wall type configuration in between them. (f) The Néel wall structure is stretched downstream on increasing the flow speed further, finally transforming into a diffused Bloch wall.

increases, the director field undergoes a deformation, accompanied by extension of the loop defect (Fig. 6.24c). The extension continues until the extended loop experiences a structural instability at higher speeds (Fig. 6.24d). The development of the instability is marked by the increasing measure of the flow-alignment of the nematic director and the corresponding reduction of $e_{1}$ and $e_{2}$. Ultimately, this results in formation of a hyperbolic hedgehog defect $(-1)$ in the pillar downstream (Fig. 6.24e) while the micron-sized pillar now mimics a virtual radial defect $(+1)$. The hyperbolic hedgehog defect, though observed to be metastable in deeper 
channels $(d>20 \mu \mathrm{m})$, is typically stable within shallow channels, and also on reversing the flow. Further increase of the flow speed results in a defect wall (Fig. 6.24), which jets out and extends over hundreds of $\mu \mathrm{m}$ downstream.

\subsubsection{Semi-integer defect loop}

In the presence of the flow, the defect loop experiences a viscous force and is pulled downstream. Tethered to the micro-pillar, the loop is progressively stretched on increasing the speed (and $E r$ ). The stretch length, $l$, was measured as the distance between the pillar center and the leading end of the defect (Fig. 6.25, inset). As plotted in Fig. 6.25, $l$ increased monotonically with the flow speed, linearly in the initial stages (upto $E r \approx 80$ ), afterwhich a non-linear dependence on $E r$ was observed. Interestingly, at comparable flow speeds, the defect loop within a shallow $((r, d, w)=(30,10,100) \mu \mathrm{m})$ channel clung too close to the pillar for optical detection (Fig. 6.26).

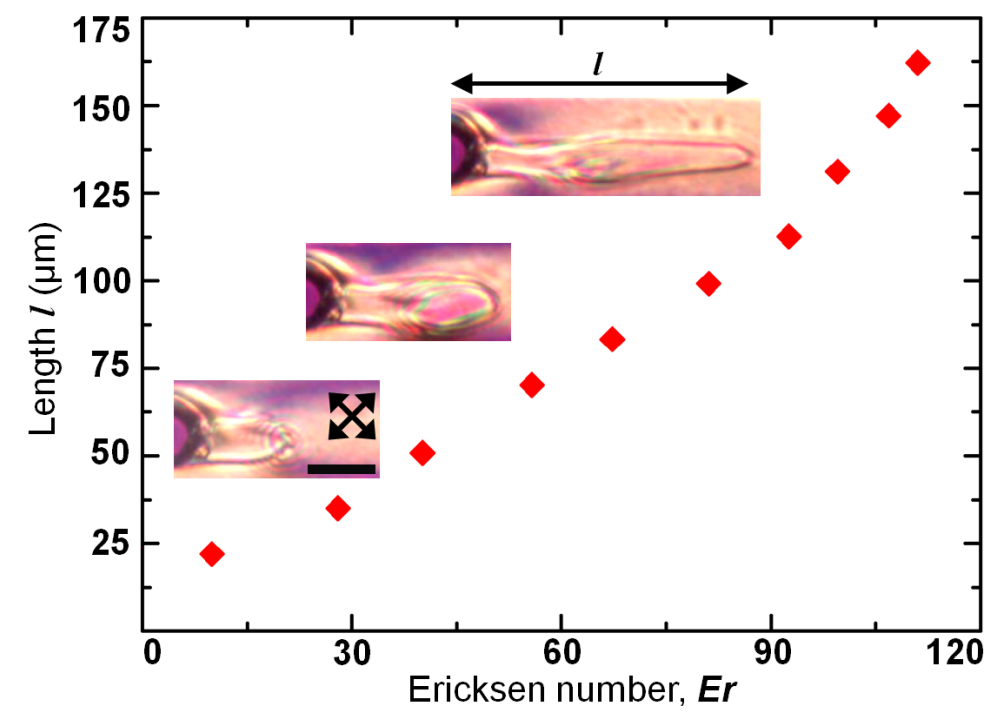

Figure 6.25: Extension of the singular loop in a $(r, d, w)=(20,40,100) \mu \mathrm{m}$ channel. The maximum length (measured between the pillar center and leading end of the defect) shows a non-linear dependence with the Ericksen number, $E r$. Inset: Micrographs show extension of the semi-integer defect loop with increasing flow speed, observed between crossed polarizers. Scale bar: $50 \mu \mathrm{m}$.

The magnitude of the director reorientation, determined by the interplay of viscous and elastic interactions, is different in the vicinity of the micro-pillar and away from it. At the 

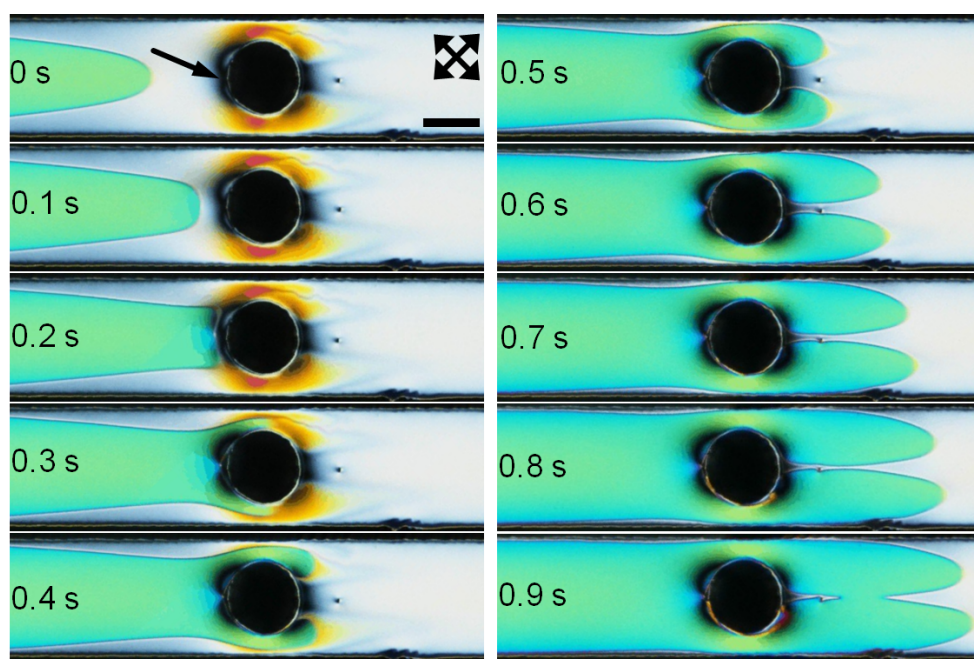

Figure 6.26: Time sequence of polarized micrographs represent the flow-alignment of the nematic director in a $(r, d, w)=(30,10,100) \mu \mathrm{m}$ channel. Arrow-head indicates the hydrodynamic stagnation point $(v=0)$. At $t=0 \mathrm{~s}$, a distinct birefringent domain (green in appearance) with a parabolic boundary is observed upstream of the micro-pillar. On the $x y$ mid-plane $(z=$ $d / 2$ ), the nematic director is oriented along the flow behind the parabolic front, whereas, ahead of it, the director is vertical. As the front moves downstream $(0<t<0.3 \mathrm{~s})$ and encounters the cylindrical pillar $(t=0.3 \mathrm{~s})$, the director around the pillar is oriented along the flow. The front however, travels with reduced speed at the center $(y=w / 2)$, seen in the time sequence $t>0.4 \mathrm{~s}$. Finally, it separates from the pillar $(t=0.9 \mathrm{~s})$ and subsequently regains the parabolic profile further downstream. Scale bar: $50 \mu \mathrm{m}$.

micro-pillar the flow is divided into two symmetric halves on either side of the pillar, each of which travel with higher local speeds. Larger reorientation of the nematic director thus follows in the region between the channel wall and micro-pillar, visualized as a distinct birefringent hue (red/yellow) in Fig. 6.26, $t=0 \mathrm{~s}$. Similarly, the symmetrical division of the flow was directly observed when a flow-aligned front (see (6) on the publication list) arrived at the pillar (Fig. 6.26, $t=0.3 \mathrm{~s}$ ). Depending upon the local shear stress, the director distortion around the pillar was minimum at the hydrodynamic stagnation point ( $v=0$, arrow-head in Fig. 6.26 and maximum at angular separations of $\pm \pi / 2$ relative to the stagnation point. The distortion however goes down once again on moving beyond $\pm \pi / 2$. Consequently, the no-flow director symmetry about the pillar axis is disrupted in the presence of the flow. With increasing speed, a flow-induced symmetry about the azimuthal $(x y)$ plane, cutting the channel into two halves at $d / 2$ (Fig. 6.24 ), begins to evolve. Experimentally, this was resolved by comparing the polarized micrographs at $t=0$ and $t=0.9 \mathrm{~s}$ in Fig. 6.26. In the latter, the director field 


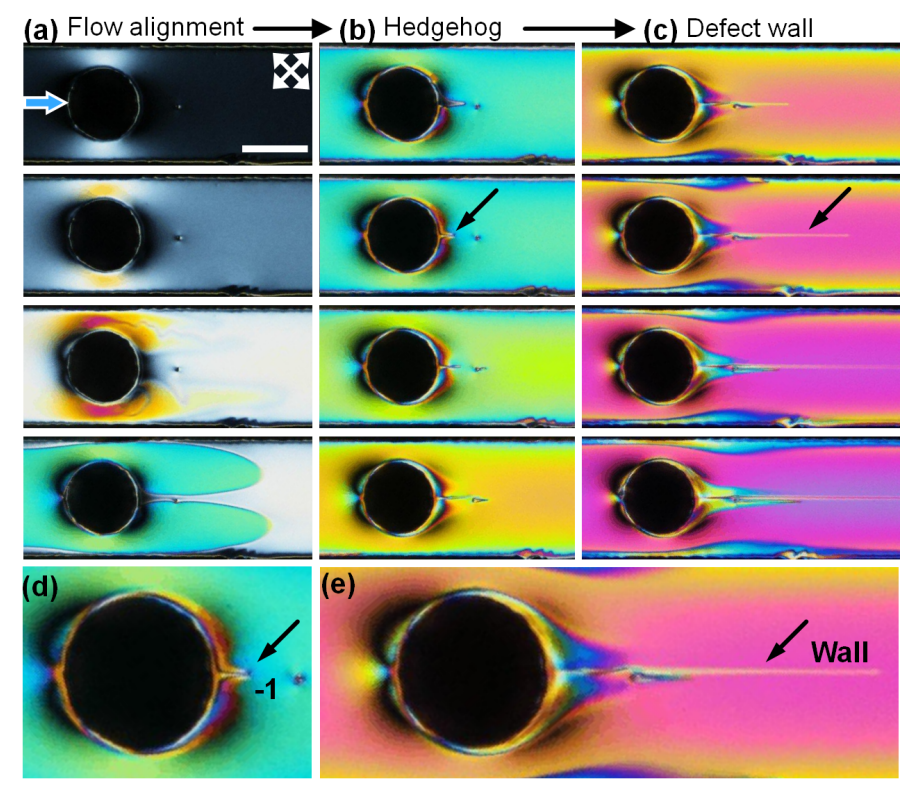

Figure 6.27: POM imaging of the flow-induced director field within a $(r, d, w)=(30,10$, 100) $\mu \mathrm{m}$ channel. (a) Flow alignment: Increasing transmission of the polarized light (top to bottom) indicates progressive reorientation of the nematic director with flow. Note that the birefringence observed between the pillar and channel walls is distinct from rest of the channel due to the high local shear rate. (b) Formation of a -1 hedgehog defect downstream of the pillar (second micrograph from top, indicated by arrow-head). The hedgehog is stretched apart from the pillar as the flow speed increases, finally resulting in the formation of the defect wall (c). Scale bar: $50 \mu \mathrm{m}$. Magnified view of the hedgehog defect (d) and the defect wall (e).

suggests the creation of a virtual +1 radial defect at the pillar center. Within deeper channels, this topological transformation is observed as an instability of the extended defect loop. A quantitative estimation of the director field and its gradient around the pillar and the stretched loop should be possible through numerical studies.

Evolution of the +1 defect is topologically balanced by the spontaneous creation of a -1 hyperbolic hedgehog defect downstream of the pillar, shown in Fig. 6.27b. The hedgehog defect is stable particularly within shallow channels (Fig. 6.27d) due to the strong influence of the anchoring induced by the surfaces. However, within a deeper channel, it is readily convected downstream and transformed into a defect wall (Fig. 6.24 , f). This phenomenon, which sets off at $v \approx 90 \mu \mathrm{m} / \mathrm{s}$ in a $40 \mu \mathrm{m}$ deep channel, qualitatively resembled the viscous jetting in microflows [238]. Similar wall defect structures are generated also within a $10 \mu \mathrm{m}$ (shallow) channel, however only at high flow speeds $v>150 \mu / \mathrm{s}$ (Fig. 6.27c, e). Deeper 
channels were suitable for the morphological investigation of the defect wall (Section 6.5.2): In addition to a better optical access to the director configuration, the defect structure evolved spontaneously even at a low flow speeds.

\subsubsection{Morphology of the wall defect}

The flow-induced director morphology of the wall defect was resolved using a combination of POM and FCPM studies as shown in Fig.6.28. A sequence of polarized micrographs shows the development of the wall defect in Fig. 6.28 a. Using high magnification POM, two distinct planes $z_{1}$ and $z_{2}$, at which the wall was focussed with maximum contrast (Fig. 6.28p), were identified. At the image plane $z_{1}$ two high contrast bright parallel lines, separated by approximately $8 \mu \mathrm{m}$, were observed. These lines meet each other at the anterior end of the defect through an angular curvature. On varying the distance between the sample and the objective, the bright lines moved out of the objective focus continuously. With the reduction of the sharp contrast, the lines now appeared diffused. However, at $z_{2}$, at a vertical separation of $\approx 12 \mu \mathrm{m}$ from $z_{1}$, a second focussed micrograph was obtained (Fig. 6.28p, bottom). The micrograph at $z_{2}$ revealed a texture distinct from the one obtained at $z_{1}$. We observed two closely placed thin parallel lines which terminated also at the anterior end of the defect. These lines, located symmetrically within the ones detected at $z_{1}$, enclosed a narrow dark region of width less than $1 \mu \mathrm{m}$ between them. Further observations carried out in white light (without the polarizers) are shown in Fig. 6.28c. Imaging at the $z_{2}$ plane revealed a bright core running through the entire length of the defect which coincided with the closely placed lines observed between crossed polarizers. The core, surrounded by darker diffused boundaries, disappeared gradually on focussing the sample back to the $z_{1}$ plane. Moreover, the above boundary coincided with the bright parallel lines observed between crossed polarizers at $z_{1}$. The white light micrographs thus suggest a lens-effect through the defect, which arises due to difference in the refractive index between the core and the outer parts of the defect.

Figure 6.28d and e show the $x y$ and $y z$ sections of the defect observed using the FCPM technique. Corresponding to the bright lines $\left(z_{1}\right.$ plane) in Fig. $6.28 \mathrm{~b}$, a parallel set of lowfluorescence-intensity lines, separated by $\approx 8 \mu \mathrm{m}$, were observed. However, a high fluorescence signal, comparable to that of the flowing nematic bulk, was obtained from the region confined between the low intensity lines. While the low fluorescence signal suggests that the LC molecules are orthogonal, the high signal indicates alignment parallel to the laser polarization direction. A cross-sectional micrograph of the defect (Fig. 6.28, additionally shows 


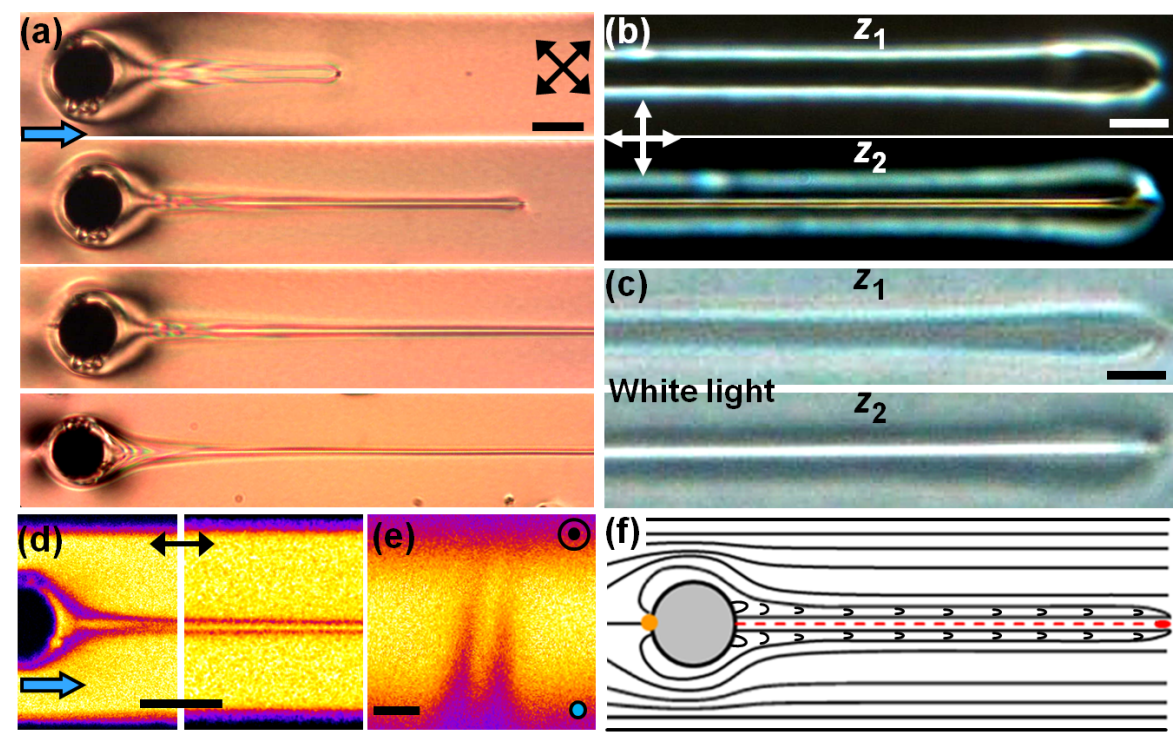

Figure 6.28: Evolution of the wall defect within a $(r, d, w)=(20,40,100) \mu \mathrm{m}$ channel. (a) Polarized micrographs show the development of the wall defect. At $E r \approx 100$ fully developed wall jets out of the pillar and extends over hundred of micrometers downstream. Scale bar: 40 $\mu \mathrm{m}$. (b) High magnification POM micrographs of the wall structure. The images were taken at two different focal planes $z_{1}$ and $z_{2}$, separated vertically by $\approx 11 \mu \mathrm{m}$. (c) Corresponding white light (without polarizers) micrographs. Image taken at $z_{2}$ shows a bright core running through the length of the defect. Scale bar: $10 \mu \mathrm{m}$. (d) Polarized fluorescence micrographs of the defect structure at the point of origin and further downstream. Direction of the laser polarization was along the flow direction in both cases (double-headed arrow). Scale bar: 50 $\mu \mathrm{m}$. (e) Cross-section ( $y z$ plane) FCPM image through the wall defect. The flow and laser polarization directions are normal to the image plane. Scale bar: $10 \mu \mathrm{m}$. (f) Schematic of the director field projection ( $x y$ plane) around the pillar, resolved using POM and FCPM imaging data.

the spread of the structure along the vertical direction. The director at the center of the wall (Fig. 6.28 p, bottom) undergoes an out-of-plane twist deformation that extends along the channel depth, resembling the so-called Bloch wall structure [26]. Combining the POM and FCPM observations, the resulting projection of the nematic director is proposed in Fig. 6.28f. In the case of an obstacle possessing spherical symmetry, the structure is expected to possess a circular cross-section. The overall structure in the present case is analogous to that of a defect stretched from a homeotropic colloid or droplet [27, 29, 236]. However, the absence of the rotational symmetry along the flow imprints a vertical extension on the singular defect arising from the micro-pillar obstacle. 


\subsubsection{Dynamics of the wall defect}
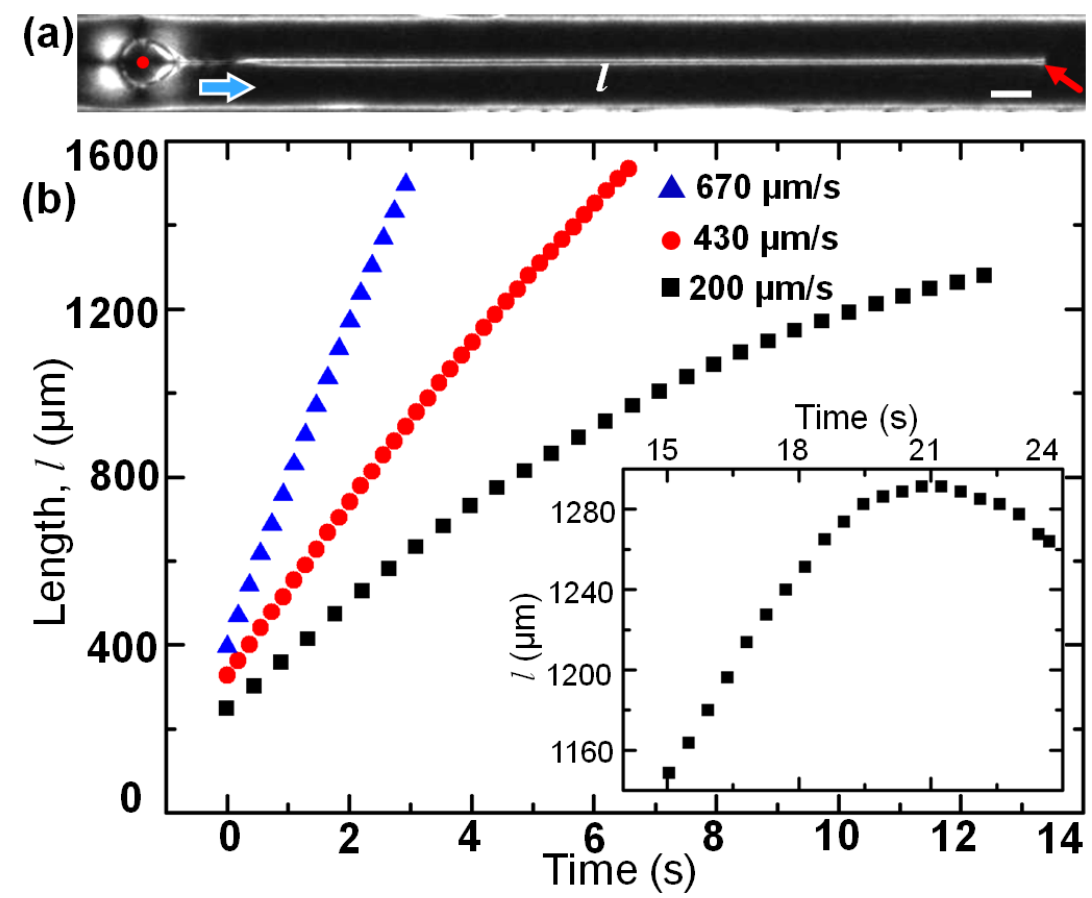

Figure 6.29: Length of the defect wall. (a) POM image of a fully developed wall stretched in the pillar downstream region (crossed polarizers with one polarizer along the flow direction). The defect length $l$ is defined as the distance between the pillar center (red dot) and the leading end of the defect (red arrow head). Scale bar: $50 \mu \mathrm{m}$. (b) The plot shows length of the defect as a function of time for three different flow rates of the nematogen. The inset shows the defect length during the late stages (shown here for $v=200 \mu \mathrm{m} / \mathrm{s}$ ). In this case it reaches a maximum of $l \approx 1300 \mu \mathrm{m}$, before snapping off. At this instance the leading end begins to move backwards.

The life span of a defect wall tethered to the micro-pillar typically lasts over a few tens of seconds. The defect life initiates with its creation, followed by stretching, and finally it terminates when the wall snaps off. Thereafter, the wall regains its length over time and the cycle is repeated. The snapped-off portion constitutes an umbilic defect [239] which is convected downstream by the flow. The posterior and anterior ends of the annihilating structure are constituted of a +1 radial and a -1 hyperbolic hedgehog defect, respectively, forming a classical umbilic defect. The umbilic possesses a rotational symmetry about axis joining the defect pair. While travelling downstream, the umbilic annihilates, as observed from the progressive reduction in its length with time. The annihilation dynamics and its dependence on 


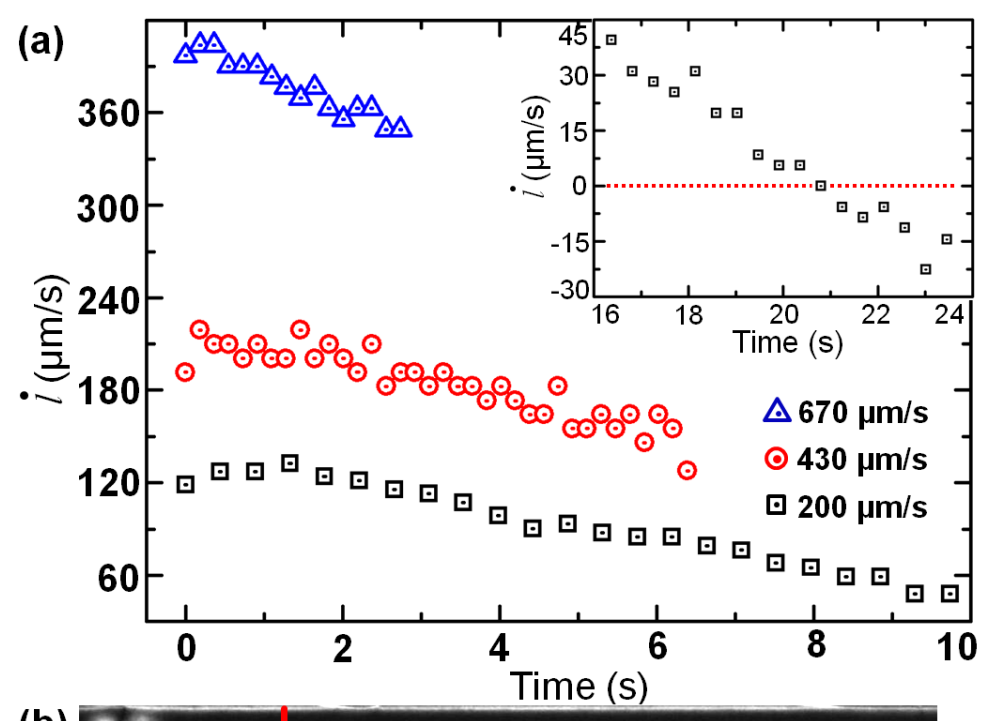

(b)

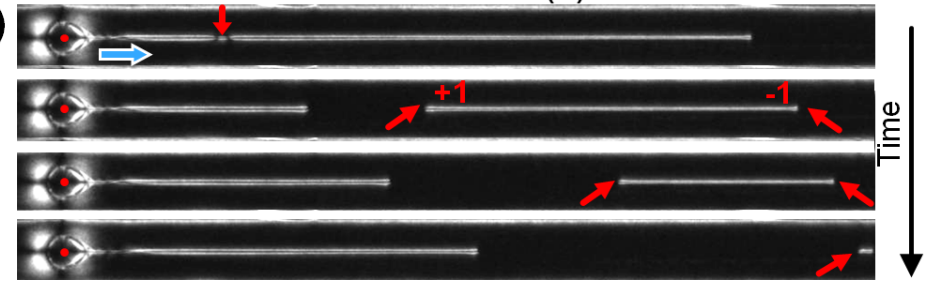

Figure 6.30: Wall velocity. (a) The plot shows the time dependence of the rate of change of the defect length, shown here for three different flow speeds. The inset shows the time dependence towards the later stages $(v=200 \mu \mathrm{m} / \mathrm{s})$. Observe the change of sign at $t \approx 21 \mathrm{~s}$. (b) Time sequence of polarized micrographs represents the process of snap-off of the defect wall. Each micrograph is separated by $1 \mathrm{~s}$, the time increasing from top to bottom. The snapoff produces an integer defect pair, which annihilates while convecting downstream. The wall originating from the pillar regains its length over time.

the flow rates is currently under investigation.

Time dependence of the length of the wall defect, $l$, was measured for three different flow speeds, as shown in Fig. 6.29. The length of the wall increased linearly during the early stages of the life-time: Over a span of $\approx 3 \mathrm{~s}$, the lengths increased by approximately $450 \mu \mathrm{m}$ (black squares), $600 \mu \mathrm{m}$ (red circles), and $1100 \mu \mathrm{m}$ (blue triangles). As time increased, the defect length gradually approached a saturation behaviour, distinctly visible in the plot corresponding to $v \approx 200 \mu \mathrm{m} / \mathrm{s}$ (Fig. 6.29 ). At higher flow rates the wall extended beyond the POM field of view. During the final stages of life, the defect reached a maximum length at $t \approx 21 \mathrm{~s}$, after which the length started to reduce. This is shown in Fig. $6.29 \mathrm{~b}$ (inset) at a flow speed of $v \approx$ $200 \mu \mathrm{m} / \mathrm{s}$. The latter marked the defect snap-off and generation of the umbilic structure. 
Figure 6.30 a plots the corresponding rate of extension of the wall $i$ as a function of time. Irrespective of the flow speed, the defect accelerated right after its creation and attained a maximum speed. The time span of the initial acceleration however shortened with increasing velocity of the nematic flow. After reaching the maximum, the wall retarded and travelled at lower speeds. $l$ decreased monotonically over time and changed its sign $(+\rightarrow-)$ in the final stages of the defect life. As shown in Fig. 6.30 (inset), $\dot{l}$ reduced continuously over $16 \mathrm{~s}<\mathrm{t}<24 \mathrm{~s}$ and changed its sign at $t \approx 21 \mathrm{~s}$. The coordinates of the sign change correspond to the maximum on the plot shown in Fig. $6.29 \mathrm{p}$, inset. Thus, at its maximum extension, the wall defect halts momentarily just prior to the snap-off upstream in the channel, indicated by the arrow-head in Fig. 6.30p (top micrograph). As seen in the sequence of micrographs, the defect wall connected to the pillar regains its length over time, while the umbilic annihilates traveling downstream.

\subsubsection{Flow reversal: Bloch wall to Néel wall transformation}

The above discussed evolution of defect structures reverse their sequence when the flow rate is gradually reduced to zero. However, an abrupt termination or a gradual reversal of the flow results in a transformation of the wall into a well-defined radial-hyperbolic defect pair. This is particularly distinct within deeper channels, as presented by the micrograph sequence in Fig. 6.31a. The transformation leads to a reduction of the wall length, and is accompanied by a modification of the POM texture (see last two micrographs in Fig. 6.31 a). The anterior end of the wall ultimately transforms into a fully-developed -1 hyperbolic hedgehog defect. Figure 6.31b and $\mathrm{c}$ show the final stages of the transformation with the pillar and channel boundaries visible (low magnification was used in this case). The topological sign of the defect was estimated by rotating the polarizers maintaining them always in crossed position. The sense of rotation of the dark extinction brushes around the defect point was found to be opposite to that of the polarizers. Additionally, the brushes around the pillar rotated in the same sense as the polarizers, confirming the +1 topological nature of the pillar center. With the formation of the hyperbolic hedgehog, the two distinct focal planes, discussed in Section 6.5.2, were no more observed. Correspondingly, in white light, the bright defect core observed previously was substituted by a point defect, which appeared dark due to scattering of light. The textural changes show that an out-of-plane director orientation within the Bloch wall transformed into an in-plane ( $x y)$ bend deformation, reminiscent of a Néel wall type configuration. The in-plane director field defining the Néel wall type configuration is schematically shown 

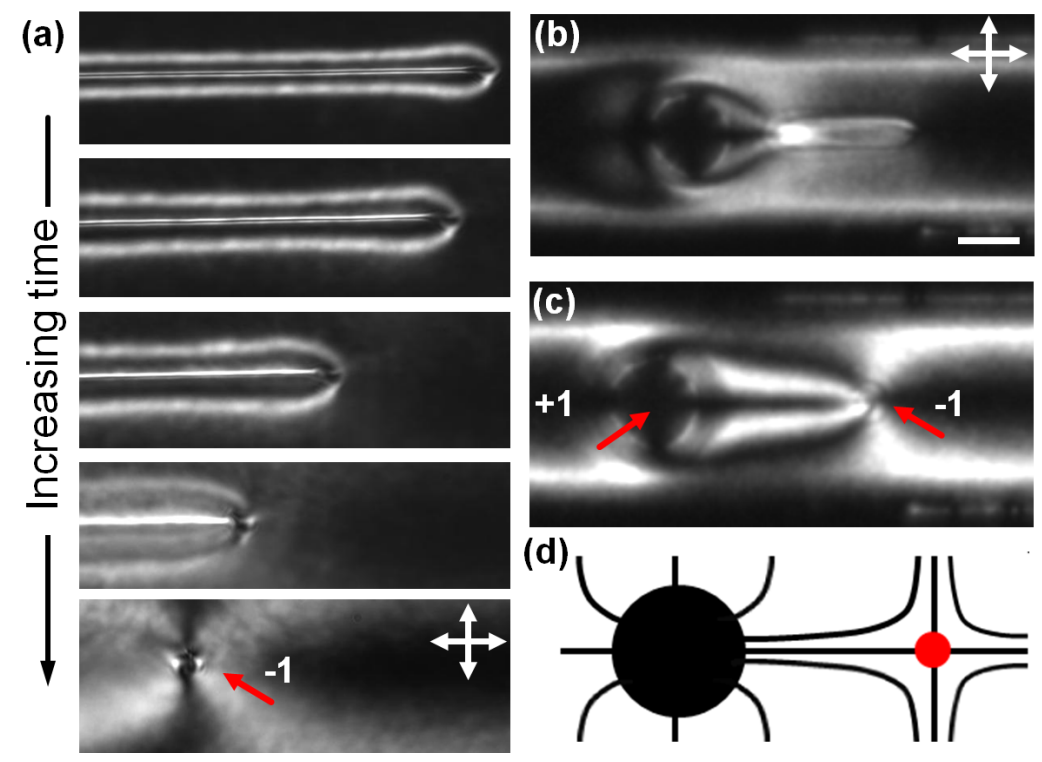

Figure 6.31: Flow reversal. (a) POM image sequence showing transformation of the wall into a hedgehog defect on gradually reversal of the flow direction. Note the accompanying textural change, especially in the last two micrographs. Micrographs in (b) and (c) show the final stages, imaged using lower magnification objective (larger field of view). Scale bar: $50 \mu \mathrm{m}$. (d) The schematic representation corresponding to a Néel wall type director configuration.

in Fig. 6.31d. Similar in-plane director fields were previously discussed in the context of the flow-induced formation of the $\pi$-walls within shallow channels possessing degenerate planar surface anchoring (see Section 6.3).

\subsubsection{Flow velocity distribution}

LCs within micro confinements can be typically described as a system of coexisting low and high molecular order species. The low ordering correspond to different kinds of singular topological defects present within the LC matrix of a relatively higher molecular ordering [26]. The presence of inclusions, e. g. colloids or droplets, within the nematic matrix leads to accompanying defects owing to localized frustration of the nematic order [24]. Consequently, the conventional technique of particle tracking turns out unsuitable for the velocity measurement within such coexisting systems. Not only do the tracer particles dispersed within the LC spontaneously form self-assembled structures [121], they also experience elasticity-mediated interactions with the defects induced by flow (see (7) on the publication list). Consequently, 

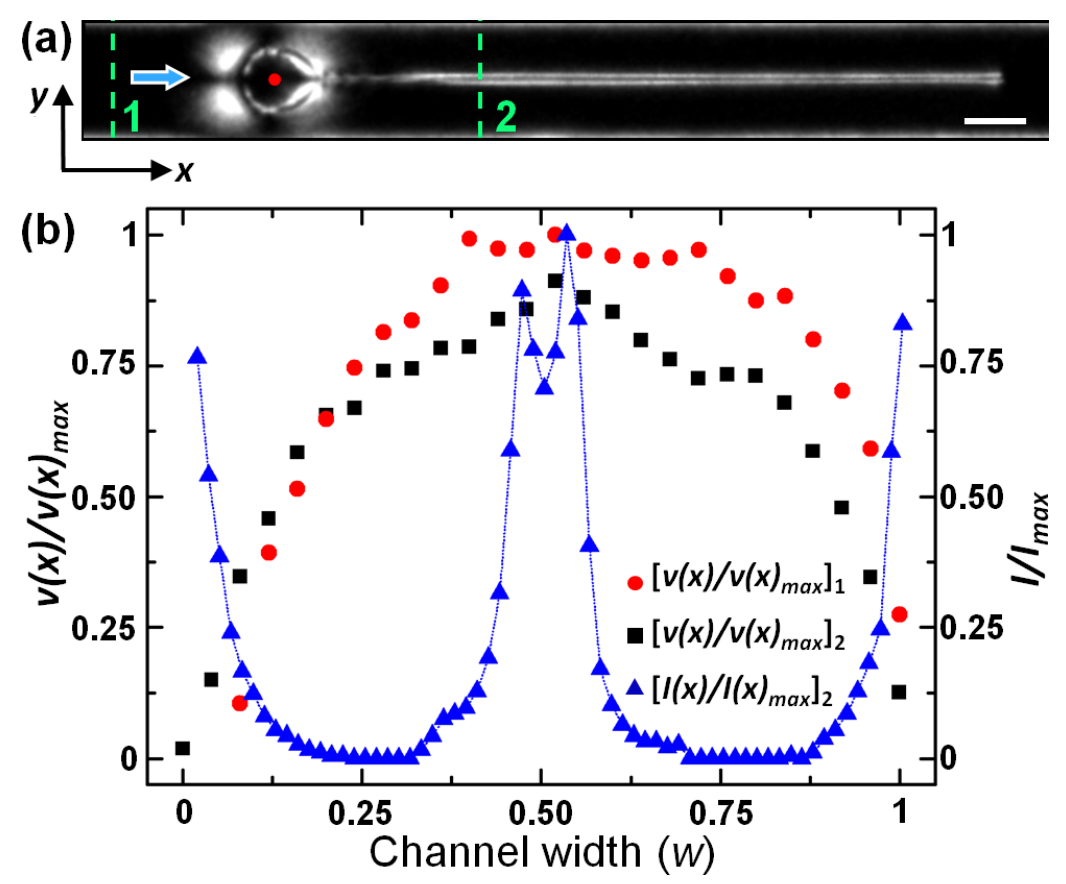

Figure 6.32: Velocity distribution across the wall defect. (a) POM image showing the pillar upstream (section 1) and pillar downstream (section 2) regions. Scale bar: $40 \mu \mathrm{m}$. (b) Normalized velocity distributions (red dot: pillar upstream, black square: pillar downstream) measured at half depth of the channel using dual-focus FCS are plotted as a function the channel width. Position of the defect wall is shown by the normalized intensity distribution across section 2 (blue triangles).

the tracer trajectory in the vicinity of a defect structure does not depict an accurate flow field, and hence is not suitable for velocimetry within coexisting phases of different ordering.

We address this experimental challenge by incorporating the dual-focus fluorescence correlation spectroscopy - 2fFCS (Chapter 3.4.4) - to measure the flow fields even in the vicinity of the defects. The velocity distribution at $z \approx d / 2$ was evaluated across the two lateral sections 1 and 2, shown in Fig. 6.32 a. Relative to the pillar, these were located $100 \mu \mathrm{m}$ behind (pillar upstream) and ahead (pillar downstream) of the pillar, respectively. Figure 6.32b shows the velocity distribution $\left(v / v_{\max }\right)$ through the sections $\mathbf{1}$ (red dots) and $\mathbf{2}$ (black squares). The velocities have been normalized with respect to the maximum velocity, $v_{\max }=456 \mu \mathrm{m} / \mathrm{s}$, obtained experimentally. Additionally, a plot of the normalized intensity distribution through the defect wall (section 2 in Fig. 6.32a) indicates the position of the latter. This is presented by the blue triangles in Fig. 6.32 b. A whole-field velocimetry using $2 \mathrm{fFCS}$ is expected to be a practi- 
cal tool for resolving the local rheological parameters in the vicinity of topological defects in flow. The velocity field measured across the defect line in Fig. 6.32 (black squares) indicates a possible enhancement of the flow speed at the center of the defect wall. Further investigations are underway to resolve the three dimensional velocity field in the vicinity of the defect line.

The above reported observations on the flow of a nematogen past a micro-sized cylindrical obstacle are first steps towards understanding the nematic flow around a fixed obstacle. Though the static director distribution was determined by the obstacle geometry, the flowinduced morphology did not exhibit any significant dependence on geometry of the pillar. The flow-induced defect structures relied strongly on the director reorientation and the consequent variation of the overall topological constraints at different stages of the flow. The fabrication of the microfluidic confinements typically yielded PDMS structures with rounded edges, which resulted in a continuous director field. However, around the pillar, singular configurations were observed at slightly perturbed states, or due to the presence of surface irregularities. The reported observations can be generalized for different combinations of $r, d$, and $w$. Though, within shallow channels $(d<10 \mu \mathrm{m})$, the formation of the singular loop is hindered, the hedgehog defect evolving subsequent to the flow-alignment is observed to be stable. In contrast, within a deeper channel $(d>20 \mu \mathrm{m})$, a well defined singular loop is observed, though the hyperbolic hedgehog structure is typically unstable. Thus, a higher flow speed was necessary to induce the hedgehog-to-wall transformation within shallow channels. Nevertheless, the defect wall was observed within the entire range of channel depth $(7<d<50 \mu \mathrm{m})$ considered in this work. Furthermore, this behaviour is supported by the nematic elasticity relation $K_{2}<1 / 2\left(K_{1}+K_{3}\right)$ [240], where $K_{1}=6.2 \mathrm{pN}$ (splay), $K_{2}=3.9 \mathrm{pN}$ (twist), and $K_{3}=8.2 \mathrm{pN}$ (bend) denote the elastic constants for nematic $5 \mathrm{CB}$. Conversely, the stability of the director transformation on flow reversal also exhibited a qualitative dependence on the channel depth, $d$. Within channels with $d<10 \mu \mathrm{m}$, the reduction of the flow speed readily converted the wall defect to a hedgehog. On the other hand, even small perturbations created within the flow were sufficient to transform the hyperbolic hedgehog configuration to a Bloch wall type structure. The Néel wall configuration was better stabilized within a channel with lower $d$. Homeotropic anchoring on the top and bottom surfaces of a shallow channel stabilized the hedgehog defect point of the Néel wall structure. Since the director easily reorients by flow in a deeper channel, the equilibrium was readily shifted towards the formation of the wall structure. It is thus expected that the flow-director interactions observed within the shallow channels can be analogously simulated even within deeper channels by enhancing the boundary effects, for instance, by increasing the strength of the homeotropic anchoring using an appropriate treatment 
of the confining surfaces. Furthermore, $2 \mathrm{fFCS}$ is demonstrated as a potential technique for velocity measurements in coexisting systems of ordered and disordered phases, thus providing an optimal platform for investigating the rheological aspects of such systems.

\subsection{Nematic flow in a hybrid microchannel}

In recent years, the field of microfluidics has seen major conceptual advances, and, enabled by the progress in methodology and technology, the number of applications has grown substantially [197-200]. In particular, it has been recognized that microfluidic devices can be beneficial - or instrumental - in a much greater range of applications as one moves away from utilizing homogeneous liquids as the transport medium. The use of emulsions, where typically aqueous droplets are embedded in an immiscible carrier fluid such as oil, has become known as droplet-based microfluidics. The approach is now widely used in essentially all fields where microfluidic devices are employed [144]. Several methods have been proposed to control the motion and position of individual droplets or other cargo within the carrier fluid by means of external fields [203-205]. The necessity of applying external fields, however, imposes important limitations in terms of design possibilities and accessible parameter ranges.

Here I propose and demonstrate a conceptually distinct yet complementary approach to guided transport of microfluidic cargo. The long-range ordering and the accompanying anisotropy in liquid crystals is utilized to controllably guide microscopic cargo on 'soft rails'. These rails are formed by topological line defects (disclination lines) within the anisotropic soft matter matrix, nematic 5CB. Consequently, the term 'soft rails' is a natural choice to christen this novel method of microfluidic transport. The general nature and behaviour of such disclination lines have been previously discussed in chapters 2.6, 2.7.3, and 5.5. The experiments presented in this section have been carried out within linear microchannels and channels with flow-bifurcation junctions, e. g. a Y-junction. The channel surfaces have been tailored to orient the LC molecules perpendicular to the PDMS walls, and parallel to the glass surface. Effectively, this offers a standard case of hybrid director alignment (Chapter 5.5). On the glass surface, the orientation of the LC molecules was orthogonal to both the velocity and its gradient along the channel depth. Methods to induce such anchoring conditions were discussed in Chapter 4.3.4. In this section we shall look into three important aspects of the topological soft rails: creation-cum-stabilization, positioning, and navigation at a channel bifurcation. Controlled creation and guidance of the disclination, typically of length $>10^{4} \mu \mathrm{m}$, have been 
achieved by tuning the coupling between the orientation and the flow. In the next chapter (Chapter 7), dedicated to flow of nematic colloids, we shall see these rails in action.

\subsubsection{Creation-cum-stabilization of the topological soft rail}

In Chapter 5.5 we have seen how the director field develops when 5CB LC equilibrated to the nematic phase at room temperature, following the interplay of surface anchoring and long-range ordering [6]. At corners formed by two PDMS walls, the alignment encounters a situation of nonconformity, which is resolved by the elastic deformations of the director field. In topological parlance, the deformations correspond to regions of topological rank $1 / 4$ at each corner. The net topological charge is conserved through the creation of a singular line defect, of opposite strength and rank 1/2 within the nematic bulk. However, in absence of any flow, the defect line collapses towards the channel corners, shown by the image sequence in Fig. 6.33 .
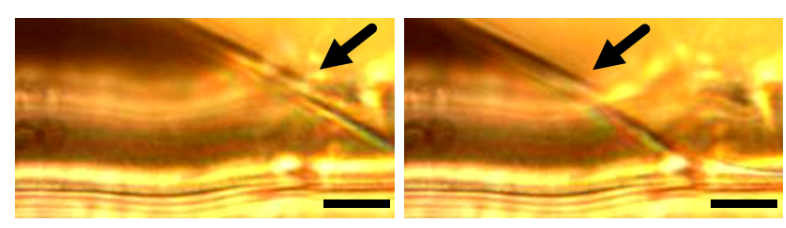

Increasing time
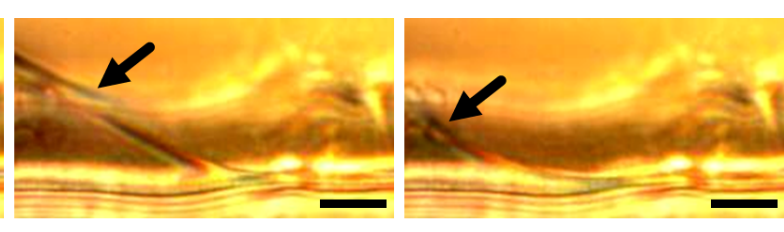

Figure 6.33: Time sequence of optical micrographs show the collapse of a disclination line towards a channel corner (top view). Time separation between images is $2 \mathrm{~s}$. Scale bar: $5 \mu \mathrm{m}$.

As suggested by the time sequence in Fig. 6.33, the bulk disclination is attracted towards a corner of the channel. The tendency of the defects to dwell near the walls can be overcome, however, by using electric fields [184] or, as demonstrated here, by the viscous drag forces resulting from a flow of the LC through the channel: At a local flow velocity of $v=8 \pm 2 \mu \mathrm{m} / \mathrm{s}$, the disclination line detached from the wall and stabilized within the flowing bulk. The elastic force $F_{\text {elastic }}$ (see Chapter 5.5) was effectively outweighed by a force $F_{\text {viscous }}$ exerted on the line by the flowing medium. This is shown in the image sequence in Fig. 6.34a. The strength of the viscous force $F_{\text {viscous }}$ was estimated to be $\approx 4 \mu \mathrm{N} / \mathrm{m}$, derived from the relations by Ryskin-Kremenetsky [116] and Imura-Okano (see Chapter 2.7.3) [241]:

$$
\left|\vec{F}_{v i s}\right|=\pi \gamma_{1} s^{2} \ln \left(\frac{3.6 K}{\gamma_{1} v r_{c}}\right)|\vec{v}|
$$



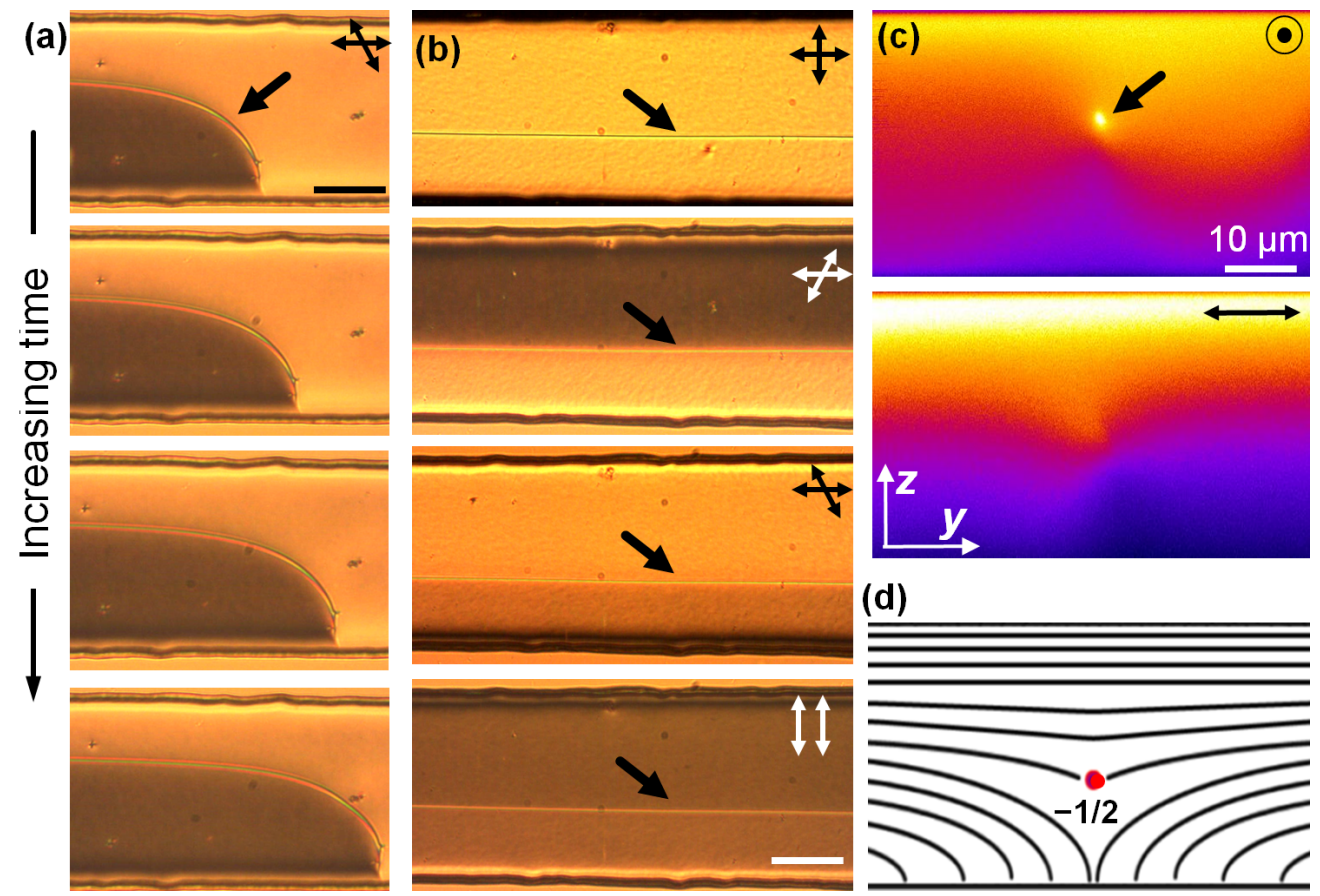

Figure 6.34: Laying down the 'soft rail'. (a) Micrograph time sequence showing a collapsed disclination line separating from the channel wall when flow is started. The black arrow points the disclination. Each image is separated by $2.5 \mathrm{~s}$. (b) POM images of the stable disclination line within the nematic bulk. The relative orientation of the polarizers are shown in each micrograph. The director has complementary angles of reorientation about the defect line. Scale bar: $50 \mu \mathrm{m}$. (c) FCPM images showing the disclination cross-section for two different laser polarizations. (d) Director field cross-section reconstructed using POM and FCPM data.

The value of the rotational viscosity of nematic $5 \mathrm{CB}$ considered for the calculations here is $\gamma_{1}=0.077$ Pas (see Table 3.2). Taking into account the uncertainties in viscosity and elastic constants, as well as the fact that the flow-director coupling was neglected, it can be concluded that the $F_{\text {viscous }}$ was strong enough to shift the static equilibrium position of the disclination line. With increasing flow speed, the disclination line was further shifted away from the wall, and was finally positioned close to the mid-plane, at $v \approx 18 \mu \mathrm{m} / \mathrm{s}$. Such a stabilized defect line is shown in shown in Fig. 6.34 p. The disclination line then stretched along the entire length of the channel (shown in Fig. 6.34a) and was found to be stable for flow velocities of up to $v \approx 200 \mu \mathrm{m} / \mathrm{s}$. The FCPM imaging across the defect cross-section (Fig. 6.34 ) supports the flow-induced director field, stabilizing the disclination line within the nematic bulk (see Fig. 6.34d). 

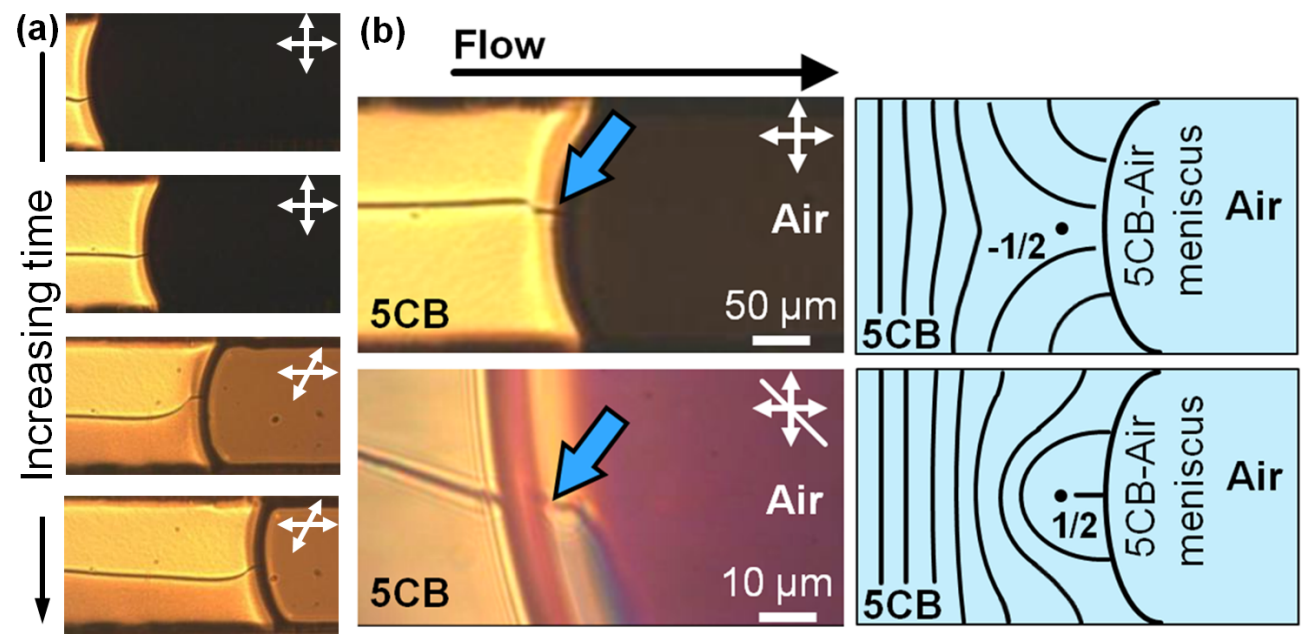

Figure 6.35: Generation of a disclination line while filling the channel. (a) Time sequence of polarized micrographs shows generation of a continuous disclination line, with the origin at the meniscus. (b) Magnified imaging close to the 5CB-air interface confirmed the origin of the disclination close to the glass surface. (c) Schematic of the possible director orientations close to the glass surface. At the 5CB-air interface, the molecules anchor perpendicularly, whereas in the upstream region, glass induces uniform planar anchoring, resulting in nucleation of the $1 / 2$ defect, which is stabilized in the upstream portion due to the prevalent surface anchoring on the channel walls.

Alternatively, the disclination line could be laid by simply filling the channel with 5CB in the nematic phase (image sequence, Fig. 6.35 a). The anchoring constraints on the channel walls and at the air-LC interface strongly support the evolution of a $1 / 2$ disclination line along the channel, as presented in Fig. 6.35 . When the channel is filled up with 5CB in the nematic phase, the LC molecules anchor perpendicularly at the interface of air and 5CB [178]. The glass surface on the other hand induces uniform planar anchoring, orthogonal to the flow direction. This leads to a conflict between the two surface-induced boundary conditions. To accommodate the antagonistic anchoring, a topological defect of rank $1 / 2$ is generated close to the nematic interface and the glass surface (Fig. 6.35b). This scenario was verified by focusing the microscope objective near the glass surface. Within the nematic bulk, sustenance of this spontaneously evolved defect is possible due to the surface anchoring present on the remaining parts of the channel walls (Fig. 6.34 ). Effectively, this leads to a continuous disclination line originating at the leading meniscus and extends through the entire length of the filled channel. Thus, by tailoring the surface and flow parameters appropriately, disclinations of arbitrary lengths can be created and stabilized within a microfluidic device. 

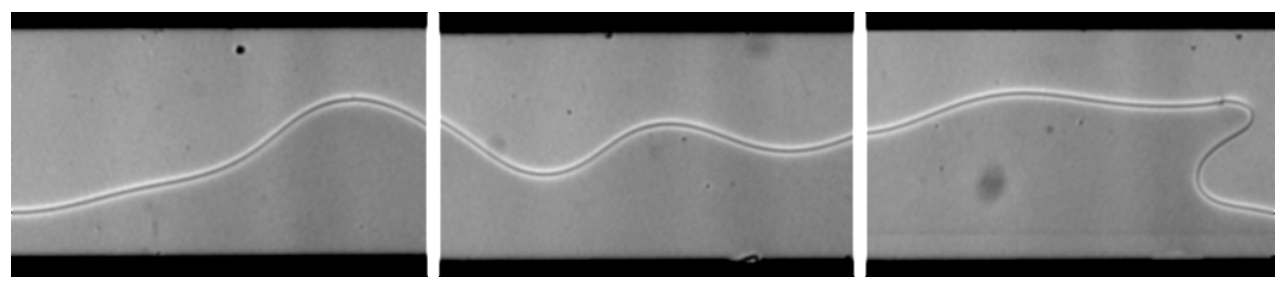

Figure 6.36: Set of polarized micrographs of a continuous disclination line within a wide microchannel $(w=500 \mu \mathrm{m}$.) The defect line is typically unstable, exhibiting a wobbly motion in the transverse direction.

Clearly, the side walls of the microchannels possessing homeotropic anchoring, have a significant contribution in stabilization of the disclination line. Due care needs to be taken for determining the appropriate combination of the flow parameters and the channel dimensions. It is plausible, that in sufficiently wide channels (high aspect ratio), the director stabilization falls short of adequate contribution from the side walls of the channel. This was observed in experiments with wide channels: In channels with $w>1000 \mu \mathrm{m}(d \approx 50 \mu \mathrm{m})$, the disclination line exhibited a wobbly motion in the transverse direction (Fig. 6.36). When similar flow speeds were applied within a channel of $w=200 \mu \mathrm{m}$, the defect line was found to be stable. Furthermore, within extremely wide channels $w>3000 \mu \mathrm{m}$, the defect was rarely observed.

\subsubsection{Navigating the topological defect at a flow bifurcation}

The disclination position in the $x y$ plane depends sensitively on flow speed. Still, the range over which the defect was shifted away from the channel wall towards the center has been rather small ( $\Delta v \approx 10 \mu \mathrm{m} / \mathrm{s}$ for a width of $200 \mu \mathrm{m})$, limiting its usefulness as a practical control parameter for positioning. The lateral position $p$ of the disclination $(0<p<0.5 w)$ can be accurately controlled, however, by varying the relative angle $\varphi(0<\varphi<90$ degree $)$ between the initial director field and the primary flow direction (Chapter 4.3.4). As plotted in Fig. 6.37, the disclination occupied a central position $(0.5 w)$ at high angles and was close to the channel wall at low angles $(\varphi \approx 20$ degree). At even lower angles, the line was too close to the wall to be detected (Fig. 6.37a, inset).

This anchoring-induced placement is crucial for controlling the position of the disclination track and offers a fresh approach to guided transport in microfluidic devices, distinct from conventional railed or field-guided transports. As shown in Fig. 6.38 a, the disclination can be placed specifically in the upper arm of the symmetrical Y-junction, where the relative angle $\varphi$ 


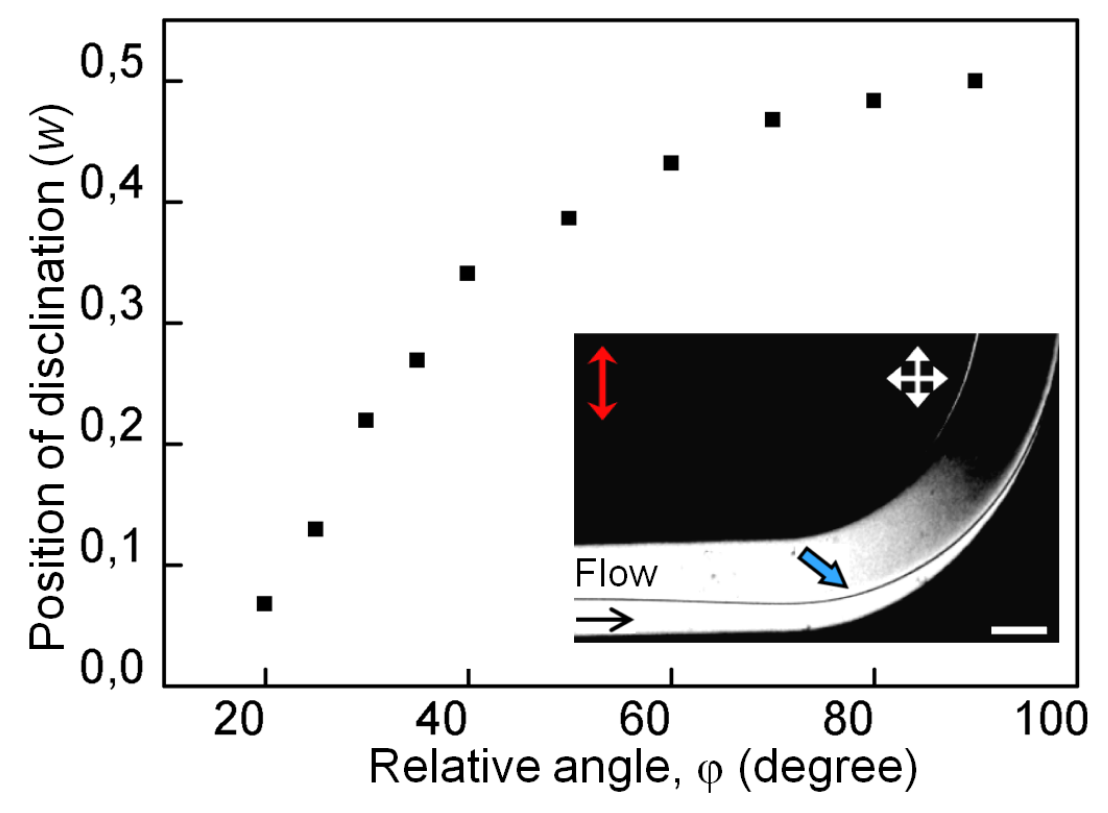

Figure 6.37: Plot representing the dependence of the disclination position on the relative angle $\varphi$ between flow direction and the initial director orientation, referenced to the channel center $(y=0.5 w)$. The polarization micrograph in the inset shows the gradual shift of the defect line (marked by the blue arrow) as $\varphi$ changes, anchoring is along the direction of the double-headed red arrow; scale bar: $100 \mu \mathrm{m}$.

is large compared with the lower arm $\left(\varphi_{\text {upper }} \gg \varphi_{\text {lower }}\right)$. When $\varphi_{\text {upper }} \approx \varphi_{\text {lower }}($ Fig. 6.38b), the line is similarly stable in either of the arms; over a large number of experiments, we observed occupation of either arms with equal likelihood. Moreover, due to the favourable anchoring shown in Fig. 6.38 , a second disclination developed in the lower arm, originating at the junction. Tailoring channel boundaries in this manner provides a route to an in situ selection of a specified target (Fig. 6.38 c). Switching of the defect line from one arm to the other was achieved through a simple flow manipulation. As shown in Fig. 6.38k, the defect line was initially directed to the upper arm. The existence of another disclination, pinned at the junction and extended in the lower arm, suggests however favourable anchoring in both the arms. The flow in the channel was first gradually reversed and then allowed to continue along the initial direction. At the instance of changing the flow direction (reversed relative to the original direction), the outlet pressure at the upper arm was marginally increased to guide the flow - and hence the disclination - to the lower arm. Once the disclination approaches the lower arm, it fuses with the hitherto pinned defect line and stabilizes there. In addition to the dis- 


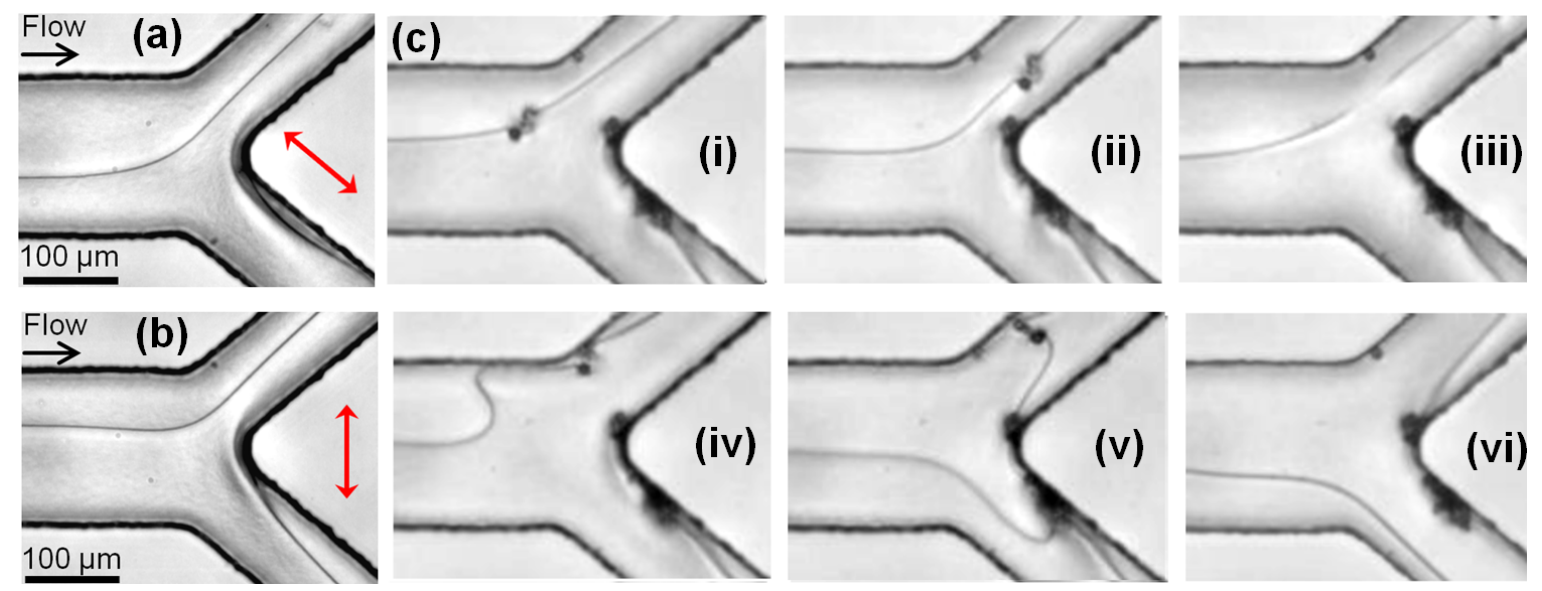

Figure 6.38: Navigating the disclination at a flow bifurcation. (a) The defect line was placed in the upper arm of a Y-junction by appropriate surface anchoring (red arrow). (b) In a confinement with symmetric director configuration, the probability of the line to occupy either arm is equal. This arrangement can be used for switching the line between the arms. (c) Image sequence showing switching of the defect from one arm to the other using a simple flow manipulation.

cussed switching technique, one can design surfaces in a way that different anchoring states are stabilized, e. g., by appropriate coating with grafted azo-benzene compounds showing photo-induced isomerization [165], or by means of micropatterned surfaces [180]. In Chapter 7.1. we shall employ the disclination for transporting colloidal cargo - particles, droplets etc. trapped by the defect line - in a guided manner to one arm of the Y-shaped microchannel. In contrast, micro-cargo transport through the rest of the nematic bulk was determined purely by the shape of the streamlines, which are symmetrically distributed relative to the junction.

The ability to navigate the disclination line is a result of the intricate coupling between flow and nematic director [15]. Close to the walls, the director adapts to the assigned boundary condition and reorients over a transition-boundary layer [6]. At higher speeds, the director undergoes flow-induced reorientation [20], which in turn generates a secondary pressure gradient in the transverse direction, due to the anisotropic viscosity and flow-director coupling [17, 23], explained in Chapter 2.7.2. The disclination line divides the channel into two longitudinal sections characterized by opposite senses of director reorientation (Fig. 6.34 $\mathrm{p}$ and c). In each sector, there is a finite pressure drop from the center towards the channel walls. The symmetry is however broken when $\varphi \neq 90$ degrees and gradients occupy asymmetrical ordinates (Fig. 6.39a). The lateral position of the disclination line is, in essence, determined 

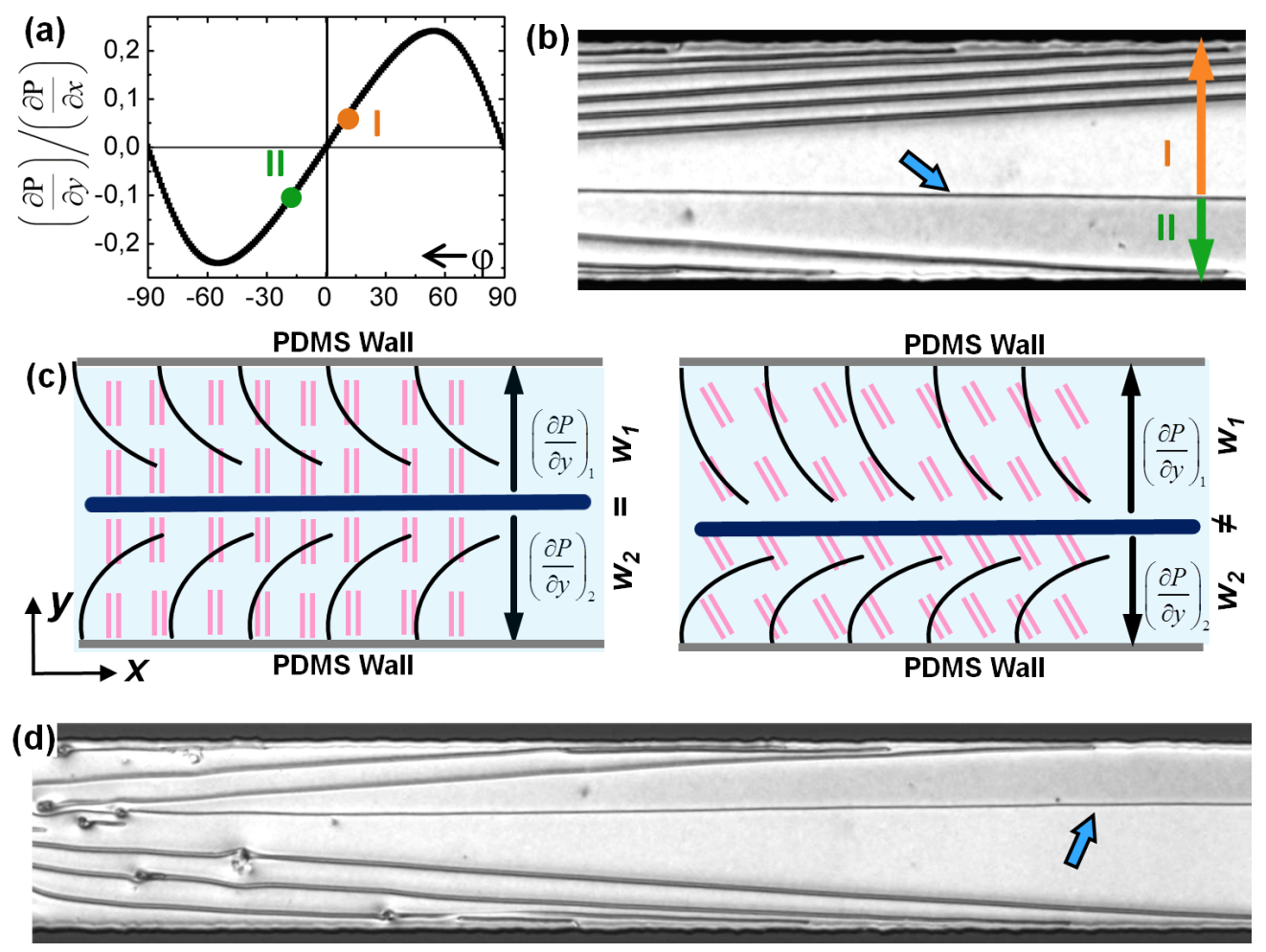

Figure 6.39: Role of secondary pressure gradient in guiding the soft rail. (a) Variation of the transverse pressure gradient with $\varphi$, calculated for 5CB and for each side of the defect line marked by the blue arrow in (b). The gradients corresponding to regions I (width $w_{1}$ ) and II (width $w_{2}$ ) are asymmetrical in this case (green and ochre points). (c) Comparative look at the symmetric and asymmetric director reorientations. (d) Defects stretched from trapped impurities reflect the secondary flow, which is a result of the transverse pressure drop from the disclination to the channel walls.

by the balance of pressure from either side,

$$
w_{1}(\partial P / \partial y)_{1}=w_{2}(\partial P / \partial y)_{2}
$$

where $w_{1}+w_{2}=w$ (Fig. 6.39 $)$.

A visual confirmation of the resulting transverse flow [17, 22] was obtained by observing the defect lines stretched out from particulate impurities trapped in the flow path. They deviated from the primary flow direction (Fig. 6.39p and d), in contrast to our observations in systems that do not favor secondary flow, e. g. within a homeotropic channel. The particulate impurity was substituted by a pillar structure, which was placed asymmetrically relative to the 
channel mid-plane. The creation and subsequent stretching of the defects at the pillar showed no deflection in the transverse direction. In fact, it was very similar to the structures we have discussed in Section 6.5. Figure 6.40 shows the polarization micrograph of a stretched defect from a pillar which was placed asymmetrically. In contrast to Fig. 6.39 b and d, in this case the defects are stretched specifically along the flow direction. This is also in agreement with the existing numerical studies by Stark and co-workers [27].

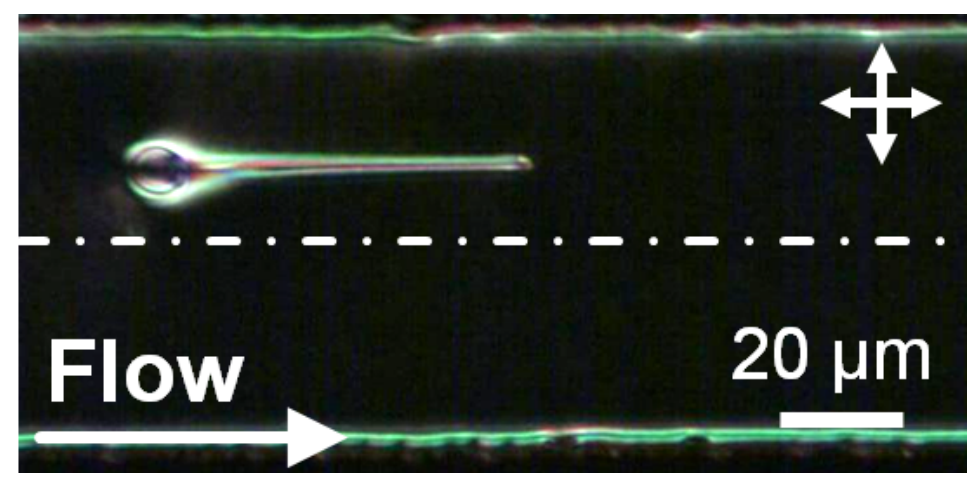

Figure 6.40: Stretching of a defect at an obstacle. Optical micrograph of a defect stretched by flow, observed between crossed polarizers. The defect was created by a micro-pillar introduced in the flow path. 5CB molecules anchor perpendicularly on all relevant surfaces.

Thus, using microfluidics, the delicate coupling between the flow and the director could be exploited to devise a novel approach to controlled creation, stabilization, and navigation of topological defect lines. These lines have been subsequently utilized to guide the transport of microfluidic cargo. On the one hand, this method complements existing techniques of guided transport; on the other hand, it offers several competitive advantages, including the possibility of in situ switching between targets and minimal design challenges. In addition to the in situ switching demonstrated here, there are also established techniques at hand to switch between different states of anchoring at the walls and thereby to navigate the disclination lines by external control [165, 180]. This approach is well suited for guiding a wide range of softmatter entities including colloidal particles and droplets of aqueous solutions, heralding the first steps towards Topological Microfluidics. 


\subsection{Transition to the chaotic regime}

Typically nematic flows at high enough Ericksen numbers lead to defect-mediated chaotic flows. In Section 6.3, the evolution of nematic textures in microfluidic environment was discussed in detail for the channels possessing degenerate planar anchoring. The spontaneous creation of flow-induced textures and defects was found to depend on the channel dimensions and flow rates. However, qualitative observations from the flow experiments suggest that the surface anchoring conditions do contribute to the transition to the defect-mediated chaotic behaviour. To demonstrate the role of surface anchoring, experiments were carried out within microchannels of similar dimensions, but with different anchoring conditions. In general, the population of the topological defects was found to be dependent not only on the flow velocity, but also on the nature of anchoring present on the microfluidic confinement. The Reynolds number, $R e$ for such micro-flows is very low (typically around $10^{-3}$ ) owing to the length scales that are involved. The other characteristic number, the Ericksen number, Er, was chosen to address the phenomena of defect-mediated chaotic behaviour. Although low Re flows are well known for being laminar, significantly chaotic flow regimes were created even in microchannels, when the NLC 5CB was flowed at high Er values. Though the detailed hydrodynamic consequence and the quantitative characterization of the system are still being investigated, in this section we shall qualitatively look at the effect of surface anchoring on the defectmediated chaotic flows. Comparative results are presented for the cases of degenerate planar, homeotropic and uniform planar surface anchoring within microchannels $150 \mu \mathrm{m}$ wide and $\approx 28 \mu \mathrm{m}$ deep.

As discussed previously, degenerate planar surface anchoring spontaneously creates surface and bulk topological defects. This is in contrast to homogeneous anchoring (homeotropic or uniform planar), in which the static equilibrium condition is generally defect free as shown in Figure 6.41a. On increasing the flow velocity and hence the $E r$, the nematic bulk flowing through the microchannel is devoid of topological defects for homogeneous surface conditions (Figure 6.41 $\mathrm{p}$ ). The defects within the channel with degenerate planar anchoring are oriented in the direction of the flow. Occasional depinning contributes to the defect population flowing downstream. With further increase of the $E r$ to $\approx 325$, the population of the defects goes up in the degenerate planar channel. Though not copious amounts, the first sustained appearance of the defects are also observed in the channels with homogeneous anchoring (Figure 6.41). However, on increasing the $E r$ further $(\approx 650)$, the channels have a distinctly different defect

population. While the channel with degenerate planar anchoring has the most, the one with 

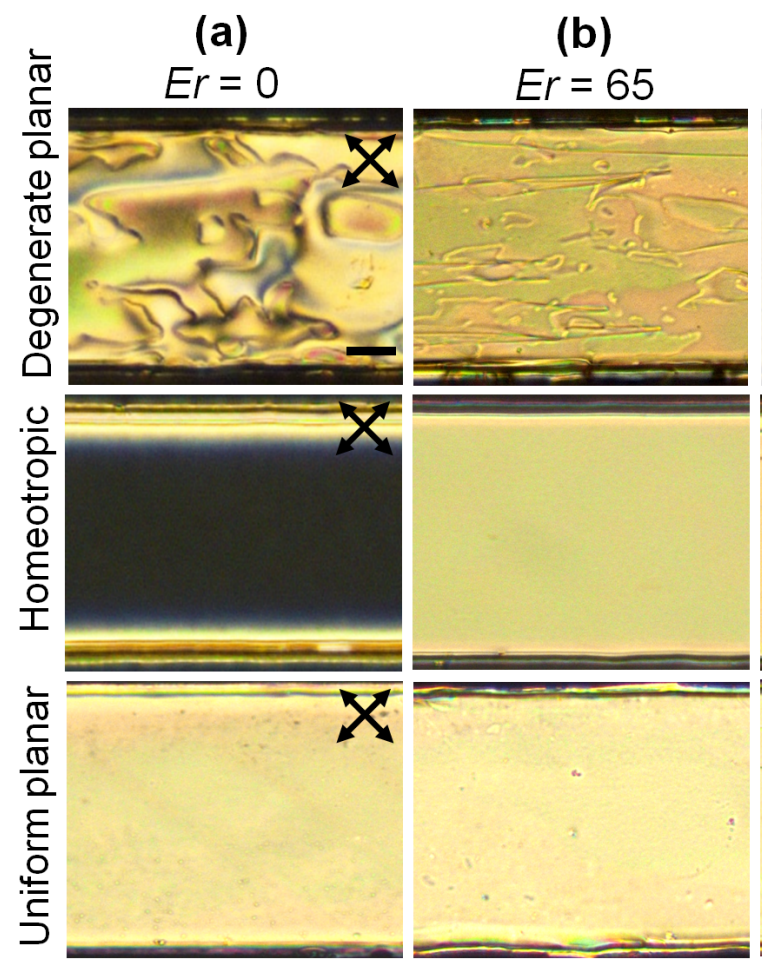

(c)

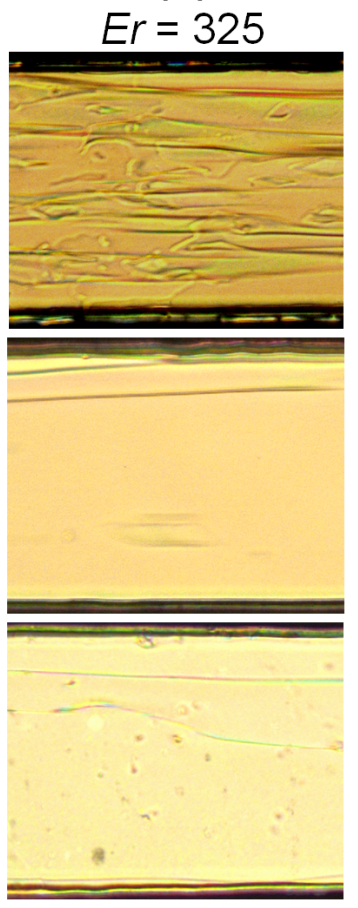

(d)

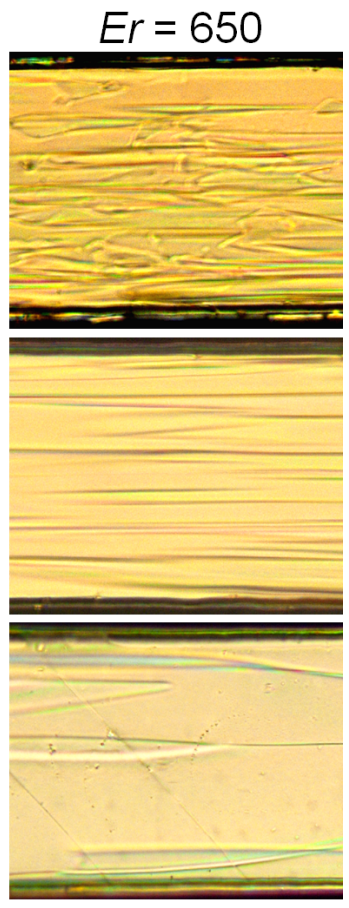

Figure 6.41: Effect of surface anchoring on topological defect population. (a) $E r=0$. Micrographs showing $5 \mathrm{CB}$ confined in degenerate planar (DP), homeotropic $(\mathrm{H})$ and uniform planar (UP) channels. In contrast to H and UP, DP shows presence of topological defects even in absence of flow. (b) $E r=65$. The defect structures pinned on DP channel surfaces are oriented by flow. $\mathrm{H}$ and UP channels do not exhibit presence of any defect structures. (c) $E r$ $=325$. DP shows a high population of defects regime. $\mathrm{H}$ and UP channels show the initial appearance of flow-induced defect structures. (d) $E r=650$. Defect population is observed to be maximum in DP and minimum in UP channel. $\mathrm{H}$ channel has intermediate value. Scale bar in the micrograph corresponds to $30 \mu \mathrm{m}$. The flow and director field are parallel in microchannel with uniform planar anchoring. The flow is from left to right in each micrograph.

uniform planar anchoring has the least defect population as is seen in Figure 6.41 d. Thus, for a given value of $E r$, it was found that the defect population was higher in a degenerate planar channel compared to channels with homogeneous anchoring, for an identical set of flow and confinement values. In shallow microchannels $(d<10 \mu \mathrm{m})$ with degenerate anchoring, the flow-induced defects experience additional stabilization by the confinement (see Section 6.3). Such defect lines, pinned at the top and/or bottom of the channel, align themselves parallel to the flow direction (Figure 6.42). The chaotic regime sets off at very high $\operatorname{Er}(\approx 1800)$ when the viscous drag on the disclinations overcomes the pinning strength, releasing the defects in 
the bulk, and subsequently transporting them downstream. The transition of defect lines from an ordered array (Figure 6.42 b) to the chaotic state shows a high degree of reversibility when the flow velocities are tuned (Figure 6.42 d-f). On heating the sample to the isotropic phase, the topological defects disappear and the stable laminar flow regime reappears.
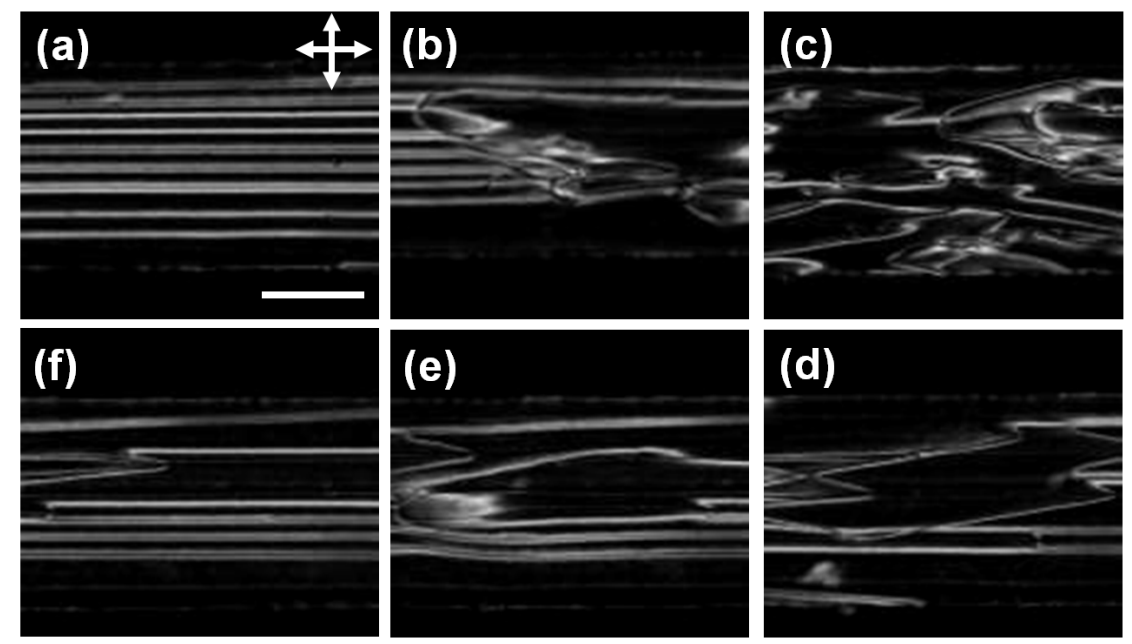

Figure 6.42: Confinement effect on defect structures. (a)-(f) Tuning the chaotic regime by flow in shallow microchannels possessing degenerate planar anchoring. The flow velocity was increased from (a) - (c) and reduced from (c) - (f). POM micrographs show the transition from an ordered array of defects (a) to a chaotic flow (c) with increasing flow velocity. On reducing the velocity the ordered defect array reappears (f). Scale bar in the micrograph corresponds to $50 \mu \mathrm{m}$. The flow is from right to left in each micrograph. 


\section{Chapter 7}

\section{Nematic colloids in microfluidic confinement}

Colloid particles dispersed within a nematic matrix offer numerous directions with regards to novel metamaterial and photonic applications. Although nematic colloids undergo elasticity mediated self-assembly with significant efficiency, additional tools like optical trapping, have enhanced the control over assembling colloidal structures of different forms and dimensions. In this chapter, we shall look into some interesting aspects of nematic colloids in flow. On one hand, we shall harness topological defects for guided transport of microfluidic cargo (colloids and droplets), on the other hand, nematic colloids were applied to extract information about the flow-induced director orientation. This chapter is expected to project some distinct capabilities of LC-based microfluidics, as compared to isotropic fluidics.

\subsection{Guided transport of microfluidic cargo on soft rails}

The growing importance of guided self-assembly, sorting and targeted delivery has led to the development of state-of-the-art droplet-based and railed microfluidics. The methods rely on flow of an isotropic carrier fluid, which is modulated using morphological patterning of the microchannels, or application of external fields. Replacing the typical isotropic fluid with an anisotropic liquid crystal provides a versatile approach to guided transport of microscopic cargo in microfluidic devices. In Chapter 6.6, we have seen that by tuning the coupling between flow and orientation of a nematic liquid crystal, topological defects in the director field could be threaded through microfluidic channels. Furthermore, the position of the defect 

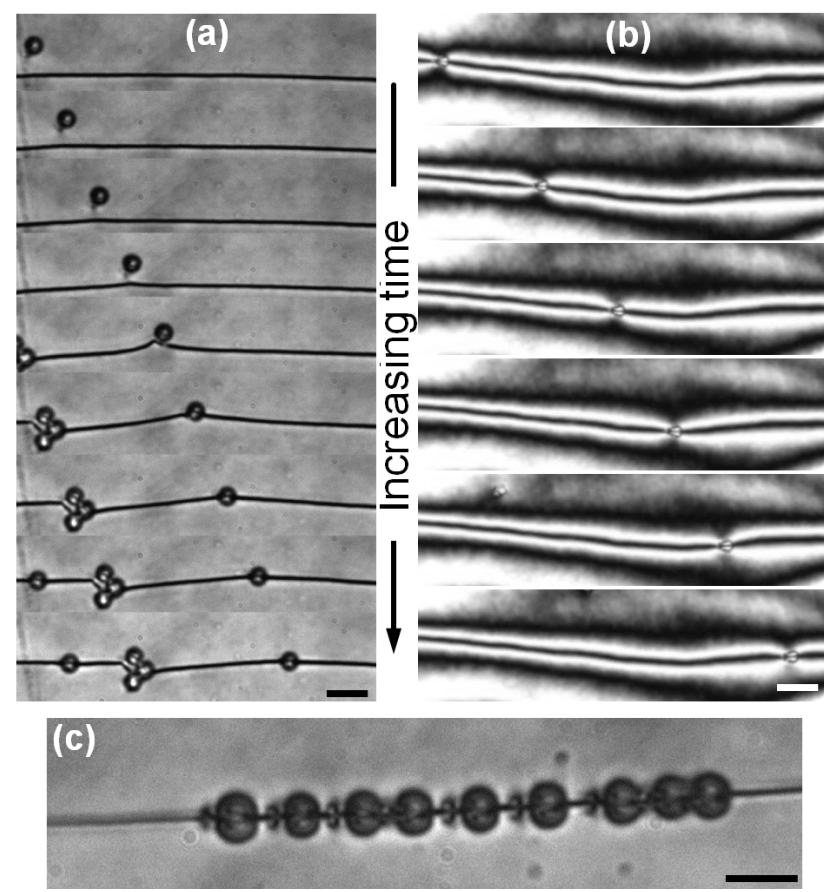

Figure 7.1: Micro-cargo transport in degenerate planar microchannels. (a) Time sequence showing the trapping of a colloidal particle to a defect line. The time difference between the micrographs is $0.1 \mathrm{~s}$. (b) Transport of a colloid particle guided through a $\pi$-wall. The time difference between the micrographs is $1 \mathrm{~s}$. (c) Colloidal chain travelling on a disclination line. Scale bar: $10 \mu \mathrm{m}$. The flow is from left to right in each case.

line was controlled through easily accessible experimental parameters. In this chapter, the defects are employed as 'soft rails' for transport of microfluidic cargo. Colloid particles and small water droplets, the 'working horses' of microfluidics, are trapped by the defect lines and consequently guided through the microfluidic device. Through appropriate surface properties discussed in the last chapter, the microfluidic cargo was successfully guided at a channel bifurcation. This introduces a unique platform for targeted delivery of single particles, droplets, or clusters of such entities, paving the way to flexible micro-cargo concepts in microfluidic settings.

The initial hints for the possible application of topological defect lines as a route to microcargo transport were observed during the flow experiments conducted within channels possessing degenerate planar surface anchoring (Chapter 6.3). Randomly formed topological defect lines were observed to trap colloidal particles or self-assembled chains, which in presence of a flow field followed the line (see Fig. 7.11, c). Even $\pi$-wall structures were observed to 
guide colloidal particles, as shown in Fig. 7.1 b. However, the major challenge encountered at this stage was the controlled generation of the topological defect lines. This was subsequently addressed through appropriate functionalization of the microchannel surfaces (Chapter 4). In chapters 5.5 and 6.6 , we have seen how defect lines can be controllably created, and even navigated at flow bifurcations.

As model cargo elements isolated colloidal particles and self-assembled chains of colloidal particles were employed. The broader applicability of this method was furthermore demonstrated by presenting the transport of aqueous droplets along the disclination lines. The colloid particle and droplets were functionalized to generate perpendicular anchoring of the 5CB molecules (Chapter 3.2). Our model cargo, bearing either a dipolar or a Saturn-ring defect (Chapter 2.8), readily formed self-assembled chains. However, both types of colloids, isolated particles as well as chains, can be transported on the soft rails. In the vicinity of a defect line, the colloids were captured by the line [134]. Following the elasticity-mediated trapping, the surrounding flow field moved the particles with the local flow velocity along the disclination track. The absolute number of dispersed entities that docked to the soft rail was increased by introducing a converging segment along the channel. One such example was shown in Fig. 6.12.

Figures $7.2 \mathrm{a}$ and $7.2 \mathrm{p}$ show a time sequence of the docking process of a colloid with Saturn-ring loop and dipolar defect, respectively. The attractive potential responsible for the capture of colloids results the from free-energy minimization in the disclination-particle system. Merging of the disclination and the Saturn-ring loop reduced the combined defect length by half the loop length. On the other hand, the free energy of the system was lowered by reducing the elastic deformation on conforming to a common director configuration between the disclination line and the dipolar defect. Similarly, colloidal chains were captured by the disclination (Fig. 7.2 c), eventually leading to the desired 'trap-and-transport' phenomenon. Water droplets of sizes similar to the colloid particles exhibit analogous anisotropic colloidal interactions [24] when dispersed within the nematic host. As shown in Fig. 7.2 d, a set of isolated droplets and a droplet chain were transported on the disclination line in a manner equivalent to that of the colloidal particles. In the present study, however, colloidal spheres were employed, because of their higher monodispersity.

The elasticity-mediated interactions exploited here - which are typically on the order of tens of $\mathrm{pN}$ [132, 134] - endow the system with high stability and robustness, as the free energy is minimized. We have also used our setup to estimate the strength of the elastic force between the particle and the disclination line, $F_{\text {disclination }}$, relative to the viscous force on a $5 \mu \mathrm{m}$ 


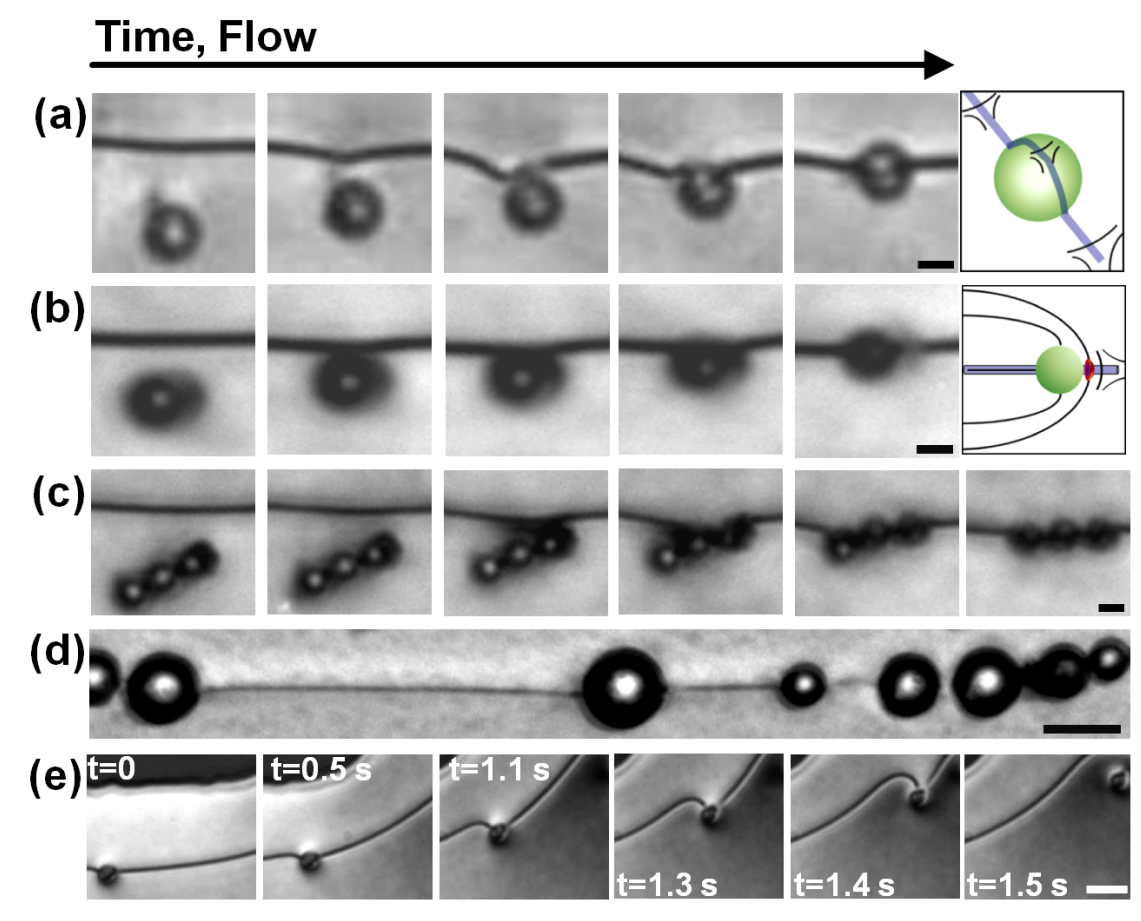

Figure 7.2: Trap-and-transport processes. Time sequence of a colloidal particle docking to the soft rail: (a) Saturn-ring type, (b) Dipolar type and (c) colloidal chain. The time increment between micrographs is $0.3 \mathrm{~s}$; scale bar: $5 \mu \mathrm{m}$. (d) Droplet rail travelling on a disclination track; scale bar: $50 \mu \mathrm{m}$. (e) Derailment of a colloid traversing a curve; scale bar: $20 \mu \mathrm{m}$.

particle (Section 7.2) and found $F_{\text {disclination }} \approx 15 \mathrm{pN}$, which is in fairly good agreement with the existing data. The balance between viscous and elastic forces is essential for the stability of the particle on the disclination track. Viscous forces greater than $F_{\text {disclination }}$ can derail the colloidal particle, for example during transport through a curved segment of the track (see the time sequence in Fig. 7.2.).

The understanding of the flow-ordering coupling has been utilized to develop a novel route to transport and guidance of microfluidic cargo. This is expected to provide an alternative, and yet a versatile platform for guided assembly of colloids, droplets, or other soft matter inclusions dispersed within an anisotropic matrix. The existing techniques of guided assembly rely heavily on guidance via 'railed microfluidics': physical grooves etched on the microchannel surface [204, 205, 242]. As a consequence, the flexibility and active control of the transported entities are severly limited. 'Soft rails' on the other hand, provide a complementary approach with distinct advantages: It is well suited for guiding a wide range of soft-matter entities, and can be easily navigated using the available experimental parameters. 


\subsection{Measurement of the particle-disclination interaction}
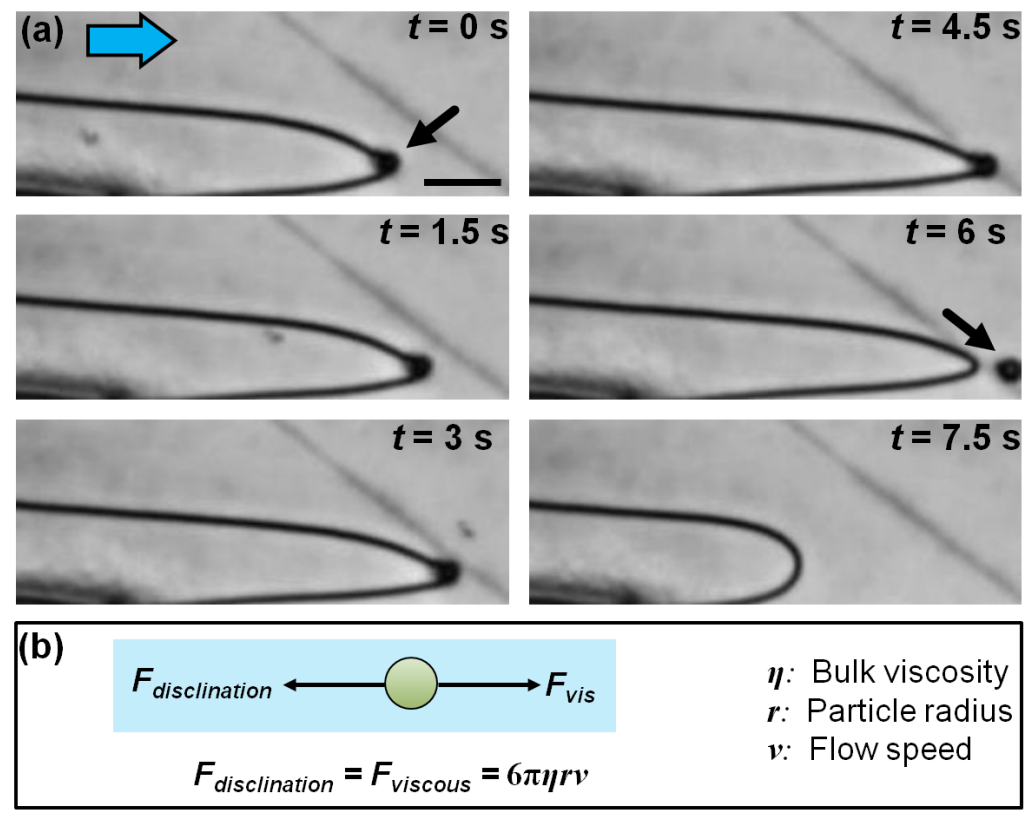

Figure 7.3: Particle-disclination interaction measurement. (a) Time sequence of a colloidal particle pulled out of a disclination line. The flow is from left to right. Balance of the viscous drag and particle-disclination interaction is used to estimate $F_{\text {disclination. }}$. Scale bar: $15 \mu \mathrm{m}$. (b) Force balance on the colloid particle at equilibrium.

The principle of measuring the particle-disclination interaction is based on the balance of the forces experienced by the colloidal particle. In presence of a flow field, the particle, typically in the Stokes regime, experiences a viscous force, $F_{v i s}$, from the flowing nematic. However, the particle is not readily dragged along by the flow, since it is trapped by the disclination line. Consequently, in a controlled experiment, the defect line is stretched by the particle in the flow direction (see image sequence in Fig. 7.3). At the maximum stretch, the particle is ultimately untrapped by the viscous force. By balancing the viscous force (Stokes drag) and strength of the elastic force between the particle and the disclination line, $F_{\text {disclination }}$ was estimated using the relations:

$$
\begin{gathered}
F_{\text {disclination }}-F_{v i s}=0 \\
F_{v i s}=6 \pi \eta r v
\end{gathered}
$$


Here $\eta$ is the bulk viscosity of nematic 5CB. The flow speed, $v$, and the radius of the colloid particle, $r$, were measured experimentally. It is noted here that the transport of colloids on the defect lines along the flow direction experience lesser resistance compared to those on the defect lines which make a finite angle with the flow direction. In the latter, the particles experience competing effects of, on the one hand, a viscous force due to flow and, on the other hand, elastic forces due to the disclination. In the limiting situation, these two effects balance each other. The strength of the particle-disclination interaction was evaluated by determining the viscous force experimentally. This was found to be $F_{\text {disclination }} \approx 15 \mathrm{pN}$ for a $5 \mu \mathrm{m}$ particle. Further investigations are ongoing to validate this technique for particles of different sizes [132].

\subsection{Director field mapping}

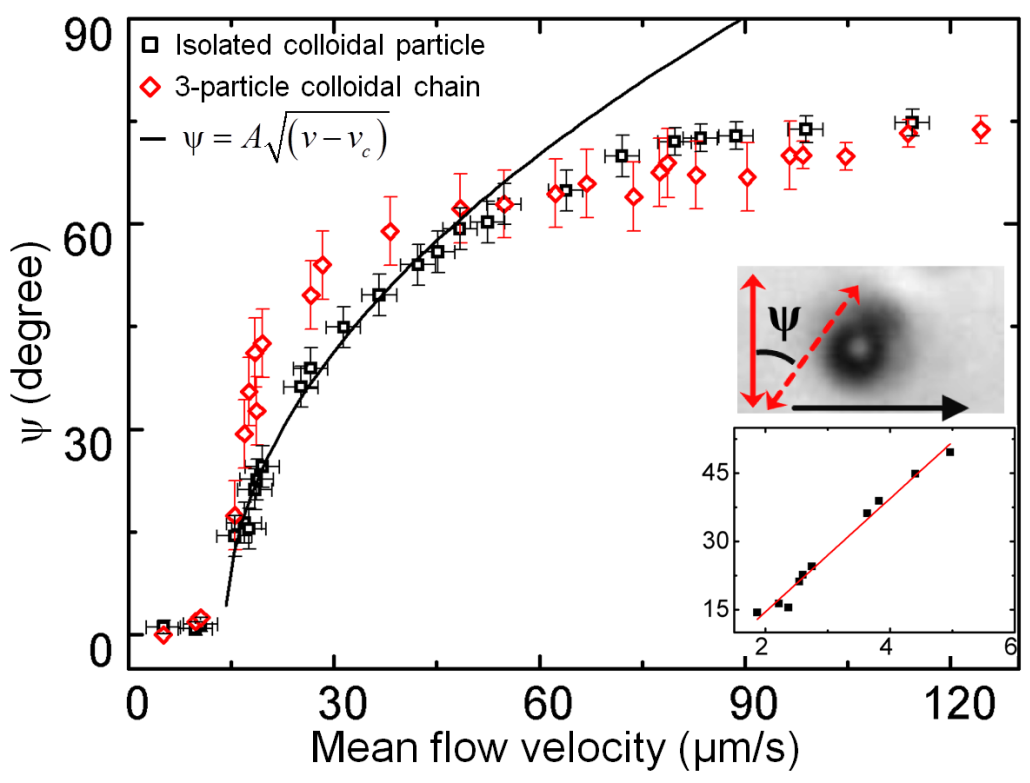

Figure 7.4: Director-field mapping by colloids in flow. Angular deviation of dipolar defects and colloidal chains, relative to the static situation (see the polarized micrograph), plotted as a function of flow speed. The inset plot shows the linear dependence of $\psi$ on $\left(v-v_{c}\right)^{1 / 2}$ (units are degrees on $y$-axis and $(\mu \mathrm{m} / \mathrm{s})^{1 / 2}$ on the $x$-axis).

It is emphasized here that the partial independence of the nematic orientation around the disclination from the direction of the topological defect line can be exploited to indepen- 
dently control the orientation of the transported particles or clusters. The orientation of any colloidal particle bearing a dipolar defect within the nematic bulk depended on the flow speed. The angular deviation relative to its static orientation, $\psi$, is plotted in Fig. 7.4 as a function of flow speed. Below $v \approx 14 \mu \mathrm{m} / \mathrm{s}$, no significant deviation was observed. However, above a threshold speed, $v_{c} \approx 15.5 \mu \mathrm{m} / \mathrm{s}$, and critical Ericksen number, $E r \approx 10$ (for $w=300 \mu \mathrm{m}$, $d=30 \mu \mathrm{m}, \mu \approx 0.06$ Pas), $\psi$ increased in a non-linear fashion and approached $\psi \approx 90$ degree at $v>123 \mu \mathrm{m} / \mathrm{s}$. This behaviour can be qualitatively explained as representing a PieranskyGuyon instability [17, 22], and reflects the usual bifurcation at the threshold speed. For $\psi<60$ degree, $\psi=A\left(v-v_{c}\right)^{1 / 2}$, where $A \approx 9.6$ degree $/(\mu \mathrm{m} / \mathrm{s})^{1 / 2}$ (Fig. 7.4 inset). Within the limits of finite width and anchoring on the channel walls, $E r$ at the threshold is in reasonable agreement with the predicted value, $E r_{c}=12.84$ [17]. Colloid chains exhibited similar dependence of their orientation on flow speed.

Flow experiments with nematic colloids were conducted also within microchannels possessing homeotropic surface anchoring. Interestingly, the colloidal transport was observed with and without flow-induced director reorientation. This is being envisaged as a route to stereo-selective transport of colloids. As shown with the help of a micrograph sequence in Fig. 7.5 a, a nematic colloid particle can be transported without any flow-induced distortion of the surrounding director field. This is confirmed by observing the variation of the typical Maltese cross appearance of the colloidal particle within a homeotropic matrix. No variation is observed in Fig. 7.5a, in which the flow speed corresponds to the weak flow regime discussed in Chapter 6.4.1. Consequently, colloidal chains, assembled parallel to the channel depth, can be transported vertically by utilizing the weak flow regime. However, as the flow speed is gradually increased, the Maltese cross pattern is distorted due to the reorientation of the surrounding director, as shown in Fig. 7.5p. It is worthwhile to mention here that the appearance of a single nematic colloid confined within homeotropic surfaces is similar to that of a colloidal chain. However, at flow speeds corresponding to the strong flow regime, the colloidal chain is oriented along the surrounding flow-aligned field, and the constituent particles can be clearly observed (Fig. 7.5b). Thus, by tuning the flow regime, the colloidal chain can be transported either vertically or horizontally. Such a behaviour is expected to hold good also for colloids with anisotropic shape, e. g. an ellipsoidal particle.

In this chapter, a novel route to guided transport of microfluidic cargo was demonstrated by exploring the combination of microfluidics, topological constraints, and elastic interactions present in nematic dispersions. On one hand, this method complements the existing techniques of guided transport, on the other hand, it offers numerous competitive advantages: 

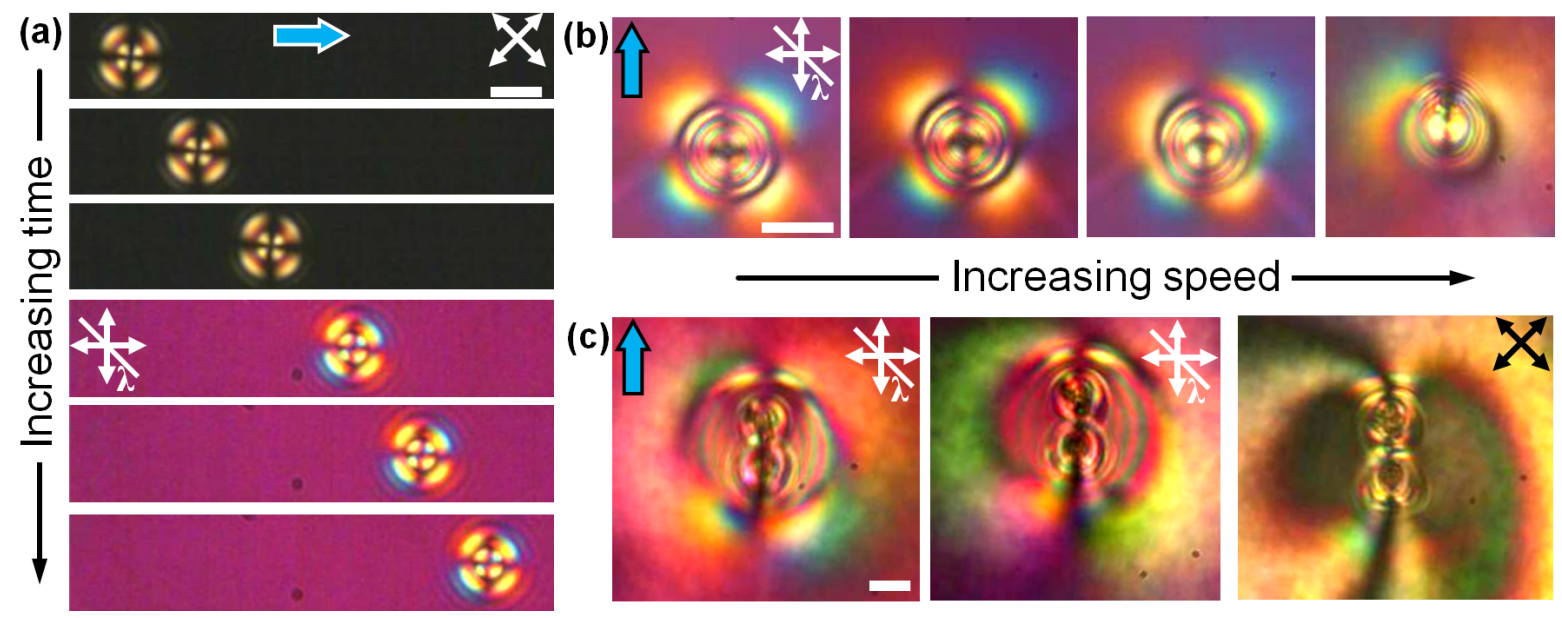

Figure 7.5: Stereo-selective transport of nematic colloids. (a) Transport of colloid in the weak flow regime within a $12 \mu \mathrm{m}$ deep channel. The flow is from left to right. Each micrograph is separated by $2.5 \mathrm{~s}$. (b) Distortion of the Maltese cross pattern as flow speed is gradually increased. The flow direction is shown by the blue arrow. (c) Two-particle chain of nematic colloids in flow. Note the gradual reorientation of the chain by increasing the flow speed (projected length of the colloid chain). In the strong flow regime the chain is horizontally aligned (rightmost micrograph). Observe the large director distortion on either side in the vicinity of the colloid chain, giving rise to a butterfly appearance. Scale bar: $5 \mu \mathrm{m}$.

in situ switching between targets, minimal design challenges, and the absence of external fields. Furthermore, the presented technique qualifies for transport of different types of dispersed phases - irrespective of the shape, size or nature - like colloids, droplets, and their self-assembled chains. The ability of the defect lines to transport droplets demonstrates the broader relevance of the 'soft rail' route, and opens up significant possibilities, especially in the domain of bio-microfluidics. LC based microfluidics was additionally presented as a tool for characterizing the disclination-particle interactions. Controlled creation and positioning of stable disclination lines $\left(>10^{4} \mu \mathrm{m}\right)$ serves as a platform for systematic study of microrheological properties of a coexisting system of low and high molecular order. Furthermore, the presented system can be explored and extended using a lyotropic LC phase, leading to the necessary compatibility with biological applications. Mapping of the director field via orientation of the colloidal defect provides a method to visualize the flow-induced ordering simply in white light microscopy, and opens up possibilities to transport colloids in a stereo-selective fashion. 


\section{Chapter 8}

\section{Ongoing research}

Since the conception of this work, several aspects of nematic micro-flows were explored which have been presented in this thesis. On one hand, I have tried to investigate the fundamental behaviour of the nematic liquid crystals flowing within strict microfluidic confinements, on the other hand, I have focused on developing some potential and practical applications based on liquid crystal microfluidics. However, with time, as the spectrum and the nature of the experiments broadened, new challenges and ideas posed ahead of me. While the challenges raise some pertinent questions for furthering the understanding of the phenomena, the ideas pertain to the exploration of LC based microfluidics as a generic platform of broader appeal and essence. Here I shall briefly present three such instances which I am currently investigating.

\section{Three dimensional velocity distribution in the vicinity of topological defects}

The core of topological defect structures signifies the region, typically of the order of nanometers, where the nematic ordering vanishes due to the strong director fluctuations. Consequently, the rheological properties at the core and its immediate vicinity, are expected to be distinct from that of surrounding defect-free matrix. In Chapter 6.5.5, we have demonstrated the application of 2fFCS (Chapter 3.4.4) as a generic velocity measurement tool for NLC flows, with or without embedded topological defects. Subsequent to the velocity measurements shown in Chapter 6.5.5, a whole-field velocity distribution around the topological defect structures is envisaged. It shall be of particular interest to additionally resolve the rheological attributes of defects of different topological definitions: integer and semi-integer line defects, and wall defect structures. 


\section{Photo-induced in situ director reorientation}

In Chapter 6.6.2, a simple flow manipulation was employed to switch the direction of a topological soft rail at flow bifurcations. Alternatively, the flow manipulation method can be substituted by reorienting the director field using light: Using NLCs doped with photosensitive dyes, the orientation of the nematic director can be precisely controlled. Ongoing experiments are directed towards the use of light to continuously vary the position of the disclination line. However, the relative strength of the flow and the light fields needs to be optimized for effecting the in situ switching by light. An alternative method of navigating the disclination line using light is to induce anchoring modifications at the surface, i. e. by tuning the surface-induced director orientation by light.

\section{Transition to defect-mediated chaotic flow}

In Chapter 6.7 we have seen the qualitative dependence of defect population on two experimetal parameters: (i) Ericksen number, and (ii) surface anchoring. Experiments and analyses are underway to extract the quanititative dependence of the defect population on the above mentioned parameters. The first question of expected relevance is the Ericksen number at which the flow becomes chaotic for each case of surface anchoring. Preliminary observations suggest that the critical Ericksen number is lower for flows within channels possessing random surface anchoring, as compared to those in channels with homogeneous anchoring (homeotropic or uniform planar). Furthermore, such chaotic behaviour may exhibit possible indications of mixing at low Reynolds numbers. 


\section{Chapter 9}

\section{Conclusion}

The work presented in this doctoral thesis spans several original findings about nematic liquid crystals and their dispersions in microfluidic environment. Microfluidic studies of ordered liquid crystalline materials have hardly been explored hitherto - either for fundamental investigations, or for applications based on LC flows. Most of the existing literature focuses on the flow of nematic LCs within very wide channels - or slits. Such investigations, in the opinion of the author, are ideal for characterizing the flow-director coupling. However, in most of the practical circumstances, we encounter channel flows: The width and depth of the channels are of similar order of magnitude. Consequently, the flow of nematogens within strict microfluidic confinements offers two interacting length scales, which can be tuned conveniently using soft lithography techniques. The present research was thus motivated by the absence of systematic investigations on nematic micro-flows in strict microfluidic confinements. The accessibility to soft lithography techniques provided a wide range of possibilities, both in terms of channel dimensions and channel geometries. Combined to it, the sensitive dependence of nematic liquid crystal $(5 \mathrm{CB})$ on surface properties and temperature, essentially provided the different sets of experimental parameters considered in this research work. The experimental observations have been analyzed by a combination of polarization optical microscopy and fluorescence confocal polarization microscopy. While particle tracking methods have been employed for flow speed measurements, dual-focus fluorescence correlation spectroscopy has been introduced in this thesis as a generic velocimetry tool for LC flows.

Controlling the surface properties is generally the first, and arguably, the most important step involved in any study of liquid crystal behaviour. Consequently, the intial challenges involved in this work were those of generating well-defined surface properties on the mi- 
crochannel surfaces. The existing techniques for inducing the desired surface properties were relevant only for macroscopic substrates. However, new methods have been devised during the course of this research to overcome this experimental challenge. Typically, freshly prepared microchannels supported degenerate planar anchoring of the nematic molecules on the channel walls. This was due to the influence of air/oxygen plasma employed for fabrication of microfluidic devices by surface-bonding of the PDMS cast and the glass substrate. Exposure to plasma resulted in a reduction of the wetting angle of $5 \mathrm{CB}$ on the confining surfaces, and generated degenerate planar anchoring. However, the channels fabricated using standard soft lithography techniques could be functionalized to produce controlled anchoring properties by using different physico-chemical treatments. For homeotropic surface anchoring, an aqueous solution of a long chain silane (DMOAP) was employed. The method generated strong and very stable homeotropic anchoring of the nematic molecules on the channel walls. Uniform planar surface anchoring was achieved by flowing a photo-sensitive polymer (PVCN-F) through the channels. The polymer chains deposited on the channel walls were subsequently exposed to polarized UV light. The exposure to the UV light oriented the chains perpendicular to the direction of the UV polarization. The resulting polymer orientation generated uniform planar surface anchoring along the UV-oriented polymer chains. Additionally, hybrid alignment of nematic LCs were achieved by inducing uniform planar anchoring on the glass (using PVA), and homeotropic surface anchoring on PDMS walls. The surface anchoring resulting from the physico-chemical treatments was generally observed to be homogeneous within the entire length of the channels. Furthermore, the techniques induced stable orientations of the nematic molecules on the surfaces, lasting over months.

Typically, the microchannels were first filled up with $5 \mathrm{CB}$ in the isotropic phase, and then allowed to cool down to the nematic phase. This registered the initial conditions for the subsequent flow experiments. Different combinations of channel geometries, dimensions, and surface anchoring conditions have been considered in this work. Rheologically, a channel with homeotropic surface anchoring offered higher hydrodynamic resistance, than one possessing uniform planar anchoring. Correspondingly, between similar pressure difference, the flow of nematic 5CB was slower in a homeotropic channel, than in a uniform planar channel. While microchannels possessing degenerate planar surface anchoring produced flow-induced defect structures, the ones possessing well-defined boundary conditions (homeotropic or uniform planar) were generally defect-free. The topological defects observed within the channels having degenerate surface anchoring were categorized into four different types: Depending upon the range of channel depths and flow speeds (hence the Ericksen number), the nature of these 
defects varied. At low Ericksen numbers, the defects comprised $\pi$-wall structures, whereas, at high Ericksen numbers, the flow was chaotic in nature due to the vigorous interaction between the topological defect structures.

Within microchannels with homeotropic anchoring, it is interesting to note that different initial equilibrium states, separated by their free energy values, were observed. The individual states could be stabilized by varying the channel aspect ratio or the temperature of the nematic phase. Although the equilibrium states were generally free of topological defects, channels possessing sharp curvatures at the corners formed by the homeotropic surfaces could lead to the spontaneous evolution singular defect lines running parallel to the channel length. Similarly, topological defect loops were observed around a cylindrical micro-pillar placed within the homeotropic channel. The intial director configuration, when perturbed by a flow field, gave way to three different flow regimes: weak, medium, and strong. The regimes are determined by the flow-director coupling, or the backflow mechanism, a key element involved in the nematodynamic analyses. The weak flow regime is characterized by minimal director distortion: The director field nearly retains the intial static orientation. On the contrary, the strong flow regime refers to complete realignment of the nematic director along the flow direction. Both the regimes lead to Poiseuille-like flow profiles. However, the medium flow regime represents a complex director configuration, resulting in a two-stream flow profile. Thus, using microchannels with appropriate aspect ratios, the Poiseuille like flow profiles were reshaped into two-stream profiles. Additionally, a temperature gradient across the channel width was used to fully steer the flow profile along the transverse direction of the microchannels. An opto-fluidic velocimetry technique was devised by replacing the linear microchannels by diverging ones. The flow of a nematic liquid crystal within the diverging channel experienced a gradient of flow speed. Consequently, a gradient of director distortion resulted, characterized by distinct birefringent colours. Using particle tracking methods, the flow speed, director distortion, and the birefringent colours were correlated. In the context of homeotropic channels, the effect of the flow-director coupling was also investigated by placing a cylindrical micropillar in the flow path. This led to a sequence of topological defects evolving at the cylindrical obstacle, each of which was distinct in nature and structure. Beginning from the static equilibrium, which led to a topological defect loop around the pillar, increasing flow speeds resulted in the director reorientation and stretching of the loop by the flow. On further increment of the flow speed, a radial-hyperbolic defect pair evolved, which then transformed into a defect wall. The sequence in which the defects evolved, reversed on gradually reducing the flow speed.

Microfluidic experiments within channels supporting hybrid director alignment provided 
a rich gamut of coexisting phenomena. In static equilibrium, a stable disclination line, extending along the channel, eventually crossed over from one side of the channel to the other. The defect line, however, could be stabilized within the nematic matrix by the presence of a flow field. Such a stable disclination line, extending over $10^{4} \mu \mathrm{m}$, was guided at a flow bifurcation. The creation, stabilization, and navigation of the disclination line, involved an intricate interplay between the viscous, elastic, and surface forces within the microfluidic confinement. Such interactions, in contrast to isotropic fluids, are unique to micro-flows of anisotropic nematic liquid crystals. The long-range ordering and consequent anisotropy in liquid crystals was then utilized to devise a novel route to guided transport of colloidal inclusions within microchannels. Isolated and self-assembled colloidal chains were transported over large distances $\left(>10^{4} \mu \mathrm{m}\right)$ with directional specificity on the 'soft rail' - the topological defect line which was created and positioned controllably within the LC matrix. This novel technique on one hand provides a generic platform for targeted delivery for different kinds of soft matter inclusions: colloids, droplets, or self-assembled chains of such entities, on the other hand it offers distinct advantages compared to microfluidics based on isotropic fluids. In comparison to the conventional 'railed microfluidics', the 'soft rail' technique is devoid of design complications and offers in situ control of the desired target. Transport of colloids through the LC bulk exhibited orientation anisotropy, which was utilized to extract information about the flow-induced director field, and propose a stereo-selective transport of colloids.

Three years of my research time went by rather quickly. I have tried to realize into experiments as many original thoughts as possible, sometimes with success, and at times without much. Numerous ideas are still confined to my note books, or awaiting experimental realization. For instance, currently I am working on a logical extension of the soft rail technique: to controllably generate the topological defects within lyotropic and/or bio-based liquid crystalline systems. Based on the suggestions from my colleagues in Stuttgart, investigations are ongoing to explore the applicability of cholesteric phase as a flowing matrix for sorting of chiral entities. However, with increasing experimental complexities, I might have cherished a little more support from the numerical community, for two reasons: (i) It provides a credible support to the experimental findings, and (ii) often numerical simulations give the experimentalists some sense of direction. Nevertheless, I am optimistic that the experts shall derive adequate inputs from this work, and thereby provide deeper numerical insights into this field than what my experiments possibly could. With the conviction that the field of liquid crystal microfluidics is at its early stages, I conclude here with the hope that this dissertation paves a way towards the highly colourful field of anisotropic fluid flows. 


\section{References}

[1] R. G. Larson, The Structure and Rheology of Complex Fluids, Oxford University Press Inc., New York, 1999.

[2] R. B. Bird, R. C. Armstrong, and O. Hassager, Dynamics of Polymeric Liquids, Vols. I, II, John Wiley and Sons, New York, 1987.

[3] A. Groisman and V. Steinberg, Nature 405, 53 (2000).

[4] A. Groisman and V. Steinberg, Nature 410, 905 (2001).

[5] Lecture Manuscripts of the $33^{\text {rd. }}$ IFF Winter School, 2002, Edited by J. K. G. Dhont, G. Gompper, and D. Richter, Forschungszentrum Jülich GmbH, Jülich, 2002.

[6] P. G. de Gennes and J. Prost, The Physics of Liquid Crystals, Oxford University Press, Oxford, 1995.

[7] P. Tabeling, Introduction to Microfluidics, Oxford University Press Inc., New York, 2005.

[8] T. M. Squires and S. R. Quake, Rev. Mod. Phys. 77, 977 (2005).

[9] Microfluidics and Microfabrication, Edited by S. Chakraborty, Springer Science+Media, New York, 2010.

[10] N-T Nguyen and S. T. Wereley, Fundamentals and Applications of Microfluidics, Artech House, Inc., MA, 2002.

[11] J. Melin and S. R. Quake, Annu. Rev. Biophys. Biomol. Struct. 36, 213 (2007). 
[12] J. L. Ericksen, Arc. Ration. Mech. Anal. 4, 231 (1960).

[13] J. L. Ericksen, Liquid Crystals and Ordered Fluids, Plenum, New York, 1970.

[14] F. M. Leslie, Q. J. Mech. Appl. Math. 19, 357 (1966).

[15] F. M. Leslie, Ration. Mech. Anal. 28, 265 (1968).

[16] O. J. Parodi, J. Phys. (Paris) 31, 581 (1970).

[17] P. Manneville and E. Dubois-Violette, J. Phys. (France) 37, 1115 (1976).

[18] P. Manneville and E. Dubois-Violette, J. Phys. (France) 37, 285 (1976).

[19] P. Manneville, J. Phys. (France) 40, 713 (1979).

[20] P. Pieranski and E. Guyon, Solid St. Comm. 13, 435 (1973).

[21] P. Pieranski and E. Guyon, Phys. Lett. 49A, 237 (1974).

[22] I. Jánossy, P. Pieranski, and E. Guyon, J. Phys. (France) 37, 1105 (1976).

[23] P. Pieranski and E. Guyon, Phys. Lett. 49A, 237 (1974).

[24] P. Poulin, H. Stark, T. C. Lubensky, and D. A. Weitz, Science 275, 1770 (1997).

[25] H. Stark, Phys. Rep. 351, 387 (2001).

[26] M. Kléman, Points, Lines and Walls: In liquid crystals, magnetic systems and various ordered media, Wiley-Interscience Publication, New York, 1983.

[27] M. Yoneya, J. Fukuda, H. Yokoyama, and H. Stark, Mol. Cryst. Liq. Cryst. 435, 75 (2005).

[28] C. Zhou, P. Yue, and J. J. Feng, J. Fluid Mech. 593, 385 (2007).

[29] S. Khullar, C. Zhou, and J. J. Feng, Phys. Rev. Lett. 99, 237802 (2007).

[30] D. Rudhardt, A. Fernandez-Nieves, D. R. Link, and D. A. Weitz, Appl. Phys. Lett. 82, 2610 (2003).

[31] B. D. Hamlington, B. Steinhaus, J. J. Feng, D. Link, M. J. Shelly, and A. Q. Shen, Liq. Cryst. 34, 861 (2007). 
[32] A. Fernandez-Nieves, D. R. Link, D. Rudhardt, and D. A. Weitz, Phys. Rev. Lett. 92, 105503 (2004).

[33] A. Fernandez-Nieves, D. R. Link, M. Marquez, and D. A. Weitz, Phys. Rev. Lett. 98, 087801 (2007).

[34] A. Fernandez-Nieves, V. Vitelli, A. S. Utada, D. R. Link, M. Marquez, D. R. Nelson, and D. A. Weitz, Phys. Rev. Lett. 99, 157801 (2007).

[35] T. Lopez-Leon and A. Fernandez-Nieves, Phys. Rev. E 79, 021707 (2009).

[36] T. Lopez-Leon, V. Koning, K. B. S. Devaiah, V. Vitelli, and A. Fernandez-Nieves, Nature Phys. 7, 391 (2011).

[37] T. Pfohl, J. H. Kim, M. Yasa, H. P. Miller, G. C. L. Wong, F. Bringezu, Z. Wen, L. Wilson, M. W. Kim, Y. Li, and C. R. Safinya, Langmuir 17, 5343 (2001).

[38] M. C. Choi, T. Pfohl, Z. Wen, Y. Li, M. W. Kim, J. N. Israelachvili, and C. R. Safinya, Proc. Natl. Acad. Sci. U.S.A. 101, 17340 (2004).

[39] S. Shojaei-Zadeh and S. L. Anna, Langmuir 22, 9986 (2006).

[40] S. Chatterjee and S. L. Anna, Phys. Rev. E 85, 011701 (2012).

[41] J. G. Cuennet, A. E. Vasdekis, L. De Sio, and D. Psaltis, Nature Phot. 5, 234 (2011).

[42] C. J. Holmes, S. L. Cornford and J. R. Sambles, Appl. Phys. Lett. 95, 171114 (2009).

[43] S. A. Jewell, S. L. Cornford, F. Yang, P. S. Cann, and J. R. Sambles, Phys. Rev. E 80, 041706 (2009).

[44] C. J. Holmes, S. L. Cornford, and J. R. Sambles, Phys. Rev. Lett. 104, 248301 (2010).

[45] P. M. Chaikin and T. C. Lubensky, Principles of Condensed Matter Physics, Cambridge University Press, Cambridge, 2003.

[46] M. Kléman and O. D. Lavrentovich, Soft Matter Physics: An Introduction, Springer, New York, 2003.

[47] L. Onsager, Annals of the New York Academy of Sciences, 51, 627 (1949). 
[48] S. Chandrasekhar, Liquid Crystals, Cambridge University Press, Cambridge, 1992.

[49] W. L. McMillan, Phys. Rev. A, 4, 1238 (1971).

[50] T. J. Sluckin, D. A. Dunmur, and H. Stegemeyer, Crystals That Flow: Classic Papers from the History of Liquid Crystals, Taylor \& Francis, London, 2004.

[51] O. Lehmann, Zeitschrift für Physikalische Chemie, 4, 462 (1889).

[52] P. Palffy-Muhoray, Physics Today, 54, September (2007).

[53] D. K. Yang and S. T. Wu, Fundamentals of Liquid Crystal Devices, John Wiley and Sons, West Sussex, 2006.

[54] I. C. Khoo, Liquid Crystals, John Wiley and Sons, New Jersey, 2007.

[55] W. Maier and A. Saupe, Zeitschrift für Naturforschung A, 15, 287 (1960).

[56] P. G. de Gennes, Phys. Lett. A, 30, 454 (1969).

[57] P. Oswald and P. Pieranski, Nematic and Cholesteric Liquid Crystals: Concepts and Physical Properties Illustrated by Experiments, Taylor \& Francis, Boca Raton, 2005, chapter B. I.

[58] U. Tkaleč, Doctoral Dissertation, Jožef Stefan International Postgraduate School, Ljubljana, 2010.

[59] P. Oswald and P. Pieranski, Nematic and Cholesteric Liquid Crystals: Concepts and Physical Properties Illustrated by Experiments, Taylor \& Francis, Boca Raton, 2005, chapter B. II.

[60] C. Blanc, D. Svenšek, S. Žumer, and M. Nobili, Phys. Rev. Lett. 95, 097802 (2005).

[61] G.P. Crawford, D. W. Allender, and J. W. Doane, Phys. Rev. A 45, 8693 (1992).

[62] G. Barbero and G. Durand, Phys. Rev. E 48, 1942 (1993).

[63] B. Jérôme, Rep. Prog. Phys. 54, 391 (1991).

[64] C. Mauguin, Comptes Rendus de lâ€TM Académie des sciences 156, 1246 (1913). 
[65] P. Oswald and P. Pieranski, Nematic and Cholesteric Liquid Crystals: Concepts and Physical Properties Illustrated by Experiments, Taylor \& Francis, Boca Raton, 2005, chapter B. V.

[66] A. A. Sonin, The Surface Physics of Liquid Crystals, Gordon and Breach, Luxembourg, 1995.

[67] A. Rapini, L. and M. Papoular, J. Phys. (Paris) Colloquium 30, C4 (1969).

[68] M. Kléman, and C. Williams, Phil. Mag. 28, 725 (1973).

[69] G. Barbero, and R. Barberi, J. Phys. (France), 44, 609 (1983).

[70] J. C. Loudet, P. Hanusse, and P. Poulin, Science 306, 1525 (2004).

[71] M. Born and E. Wolf, Principles of Optics, Sixth Edition, Pergamon Press, Oxford, 1980.

[72] I. Dierking, Textures of Liquid Crystals, Wiley-VCH Verlag, Weinheim, 2003.

[73] M. Miesowicz, Nature 158, 27 (1946).

[74] Ch. Gähwiller, Mol. Cryst. Liq. Cryst. 20, 301 (1973).

[75] I. W. Stewart, The static and dynamic continuum theory of liquid crystals, Taylor and Francis, London and New York, 2004.

[76] P. Oswald and P. Pieranski, Nematic and Cholesteric Liquid Crystals: Concepts and Physical Properties Illustrated by Experiments, Taylor \& Francis, Boca Raton, 2005, chapter B. III.

[77] G. P. Alexander, B. G. Chen, E. A. Matsumoto, and R. D. Kamien, Rev. Mod. Phys. 84, 497 (2012).

[78] Y. Bouligand, J. Phys. (Paris) 35, 215 (1974).

[79] N. D. Mermin, Rev. Mod. Phys. 51, 591 (1979).

[80] H. R. Trebin, Adv. Phys. 31, 195 (1982). 
[81] G. E. Volovik, The Universe in a Helium Droplet, Oxford University Press, Oxford, 2003.

[82] A. J. Bray, Adv. Phys. 51, 481 (2002).

[83] S. Chandrasekhar and G. Ranganath, Adv. Phys. 35, 507 (1986).

[84] S. D. Sarma and A. Pinczuk, Perspectives in Quantum Hall Effects, Wiley-Interscience, Oxford, New York, 1996.

[85] I. Chuang, R. Durrer, N. Turok, and B. Yurke, Science 251, 1336 (1991).

[86] I. Chuang, N. Turok, and B. Yurke, Phys. Rev. Lett. 66, 2472 (1991).

[87] O. Lehmann, Flüssige Kriistalle, Verlag von Wilhelm Engelmann, Leipzig, 1904.

[88] O. Lehmann, J. Phys. 8, 713 (1909).

[89] O. Lehmann, Bull. Soc. Franc. Miner. 33, 300 (1910).

[90] F. Grandjean, Bull. Soc. Franc. Miner. 29, 164 (1916).

[91] F. Grandjean, C. R. Acad. Sci. (Paris) 172, 71 (1921).

[92] G. Friedel, Ann. Phys. (Paris) 18, 273 (1922).

[93] O. Lehmann, Die Scheinbar lebenden Kristalle, Esslingen, 1907.

[94] F. C Frank, Disc. Faraday Soc. 25, 19 (1958).

[95] G. S. Ranganath, Mol. Cryst. Liq. Cryst. 87, 187 (1982).

[96] C. Fan, Phys. Lett. 34A, 335 (1971).

[97] N. V. Madhusudana and R. Pratibha, Mol. Cryst. Liq. Cryst. 89, 249 (1982).

[98] P. Oswald and P. Pieranski, Nematic and Cholesteric Liquid Crystals: Concepts and Physical Properties Illustrated by Experiments, Taylor \& Francis, Boca Raton, 2005, chapter B. IV.

[99] R. B. Meyer, Phil. Mag. 27, 405 (1973).

[100] G. G. Peroli and E. G. Virga, Phys. Rev. E 54, 5235 (1996). 
[101] V. Freedericksz and V. Zolina, Trans. Faraday Soc. 29, 919 (1933).

[102] V. Freedericksz and V. Tsvetkov, Phys. Z. Soviet Union 6, 490 (1934).

[103] R. B. Meyer, Phys. Rev. Lett. 22, 918 (1969).

[104] W. J. A. Goossens, Liq. Cryst. 5, 1083 (1989).

[105] B. Ya Zel'dovich, N. V. Tabiryan and Yu S. Chilmgaryan, Sov. Phys. JETP 54, 32 (1981).

[106] S. D. Durbin, S. M. Arakelian, and Y. R. Shen, Phys. Rev. Lett. 47, 1411 (1981).

[107] H. L. Ong, Phys. Rev. A 28, 2393 (1983).

[108] J. L. Ericksen, Phys. Fluids 9, 1205 (1966).

[109] D. Foster, T. Lubensky, P. Martin, J. Swift, and P. Pershan, Phys. Rev. Lett. 26, 1016 (1971).

[110] P. Martin, O. Parodi, and P. Pershan, Phys. Rev. A 6, 2401 (1972).

[111] F. Jähnig and M. Schmidt, Ann. Phys. 71, 129 (1971).

[112] D. Svenšek, Doctoral Dissertation, Faculty of Mathematics and Physics, University of Ljubljana, Ljubljana, 2003.

[113] F. M. White, Fluid Mechanics, McGraw-Hill, New York, 2006.

[114] G. Toth, C. Denniston, and J. M. Yeomans, Phys. Rev. Lett. 88, 105504 (2002).

[115] D. Svenšek and S. Žumer, Phys. Rev. E 66, 021712 (2002).

[116] G. Ryskin and M. Kremenetsky, Phys. Rev. Lett. 67, 1574 (1991).

[117] D. Svenšek and S. Žumer, Continuum Mech. Thermodyn 14, 231 (2002).

[118] I. Dierking, O. Marshall, J. Wright, and N. Bulleid, Phys. Rev. E 71, 061709 (2005).

[119] I. Dierking, M. Ravnik, E. Lark, J. Healey, G. P. Alexandar, and J. M. Yeomans, Phys. Rev. E 85, 021703 (2012).

[120] R. W. Ruhwandl and E. M. Terentjev, Phys. Rev. E 55, 2958 (1997). 
[121] I. Muševič, M. Škarabot, U. Tkalec, M. Ravnik, and S. Žumer, Science 313, 954 (2006).

[122] O. V. Kuksenok, R. W. Ruhwandl, S. V. Shiyanovskii, and E. M. Terentjev, Phys. Rev. E 54, 5198 (1996).

[123] M. Zapotocky, L. Ramos, P. Poulin, T. C. Lubensky, and D. A. Weitz, Science 283, 209 (1999).

[124] C. P. Lapointe, T. G. Mason and I. I. Smalyukh, Science 326, 1083 (2009).

[125] M. Humar, M. Ravnik, S. Pajk, and I. Musevic, Nature Phot. 3, 595 (2009).

[126] J-B. Fleury, D. Pires, and Y. Galerne, Phys. Rev. Lett. 103, 267801 (2009).

[127] G. M. Koenig Jr., I- H. Lin, and N. L. Abbott, Proc. Natl. Acad. Sci. U.S.A. 107, 3998 (2010).

[128] M. Ravnik, G. P. Alexandar, J. M. Yeomans, and S. Žumer, Proc. Natl. Acad. Sci. U.S.A. 108, 5188 (2011).

[129] P. E. Cladis, M. Kleman, and P. Pieranski, C. R. Acad. Sci. (Paris) 273B, 275 (1971).

[130] E. M. Terentjev, Phys. Rev. E 51, 1330 (1995).

[131] Y. Gu and N. L. Abbott, Phys. Rev. Lett. 85, 4719 (2000).

[132] P. Poulin, V. Cabuil, and D. A. Weitz, Phys. Rev. Lett. 79, 4862 (1997).

[133] I. Muševič, M. Škarabot, and M. Humar, J. Phys. Condens. Matter 23, 284112 (2011).

[134] D. Pires, J-B. Fleury, and Y. Galerne, Phys. Rev. Lett. 98, 247801 (2007).

[135] A. Martinez,H. C. Mireles, and I. I, Smalyukh, Proc. Natl. Acad. Sci. U.S.A. 108, 20891 (2011).

[136] The Landolt-Börnstein Database, Springer Materials VIII/5A, DOI: 10.1007/10694796-63 (2003).

[137] P. P. Karat and N. V. Madhusudana, Mol. Cryst. Liq. Cryst. 36, 51 (1976).

[138] K. Skarp, S. T. Lagerwall, and B. Stebler, Mol. Cryst. Liq. Cryst. 60, 215 (1980). 
[139] A. G. Chmielewski, Mol. Cryst. Liq. Cryst. 132, 339 (1986).

[140] M. Cui and J. R. Kelly, Mol. Cryst. Liq. Cryst. 331, 49 (1999).

[141] Material properties available on the website of microparticles GmbH, www.microparticles.de.

[142] F. Kahn, G. N. Taylor, and H. Schonhorn, Proc. IEEE. 61, 823 (1973).

[143] J. M. Brake, A. D. Mezera, and N. L. Abbott, Langmuir 19, 6436 (2003).

[144] R. Seemann, M. Brinkmann, T. Pfohl, and S. Herminghaus, Rep. Prog. Phys. 75, 016601 (2011).

[145] G. M. Whitesides, Nature 442, 368 (2006).

[146] J. C. McDonald and G. M. Whitesides, Acc. Chem. Res. 35, 491 (2002).

[147] J. N. Lee, C. Park, and G. M. Whitesides, Anal. Chem. 75, 6544 (2003).

[148] Microscopy Resource Center, available on the website of Olympus, www.olympusmicro.com.

[149] W. D. Nesse, Introduction to Optical Minerology, Oxford University Press Inc., New York, 2004.

[150] B. L. Van Horn and H. H. Winter, Appl. Opt. 40, 2089 (2001).

[151] P.G. de Gennes, The Physics of Liquid Crystals, Clarendon Press, Oxford 1974.

[152] O. D. Lavrentovich, Pramana 61, 373 (2003).

[153] M. Minsky, Memoir on inventing the confocal microscope, Scanning 10, 128 (1988).

[154] M. Böhmer and J. Enderlein, Single-Molecule Detection in Solution - Methods and Applications, VCH-Wiley, Berlin/New York, 2002.

[155] R. D. Goldman and D. L. Spector, Live Cell Imaging: A Laboratory Manual, Cold Spring Harbor Press, New York, 2005. 
[156] D. Semwogerere and E. R. Weeks, Encyclopedia of Biomaterials and Biomedical Engineering, Chapter on Confocal Microscopy, Taylor and Francis, DOI: 10.1081/E-EBBE$120024153,2005$.

[157] I. I. Smalyukh, S. V. Shiyanovskii, and O. D. Lavrentovich, Chem. Phys. Lett. 336, 88 (2001).

[158] Fluorescence Confocal Polarizing Microscopy, available at www.lci.kent.edu/Lavrentovich/Group.html.

[159] I. Dierking, Chem. Phys. Chem. 2, 663 (2001).

[160] I. Jánossy, Phys. Rev. E 49, 2957 (1994).

[161] H. Tajalli, A. G. Gilani, M. S. Zakerhamidi, and P. Tajalli, Dyes and Pigments 78, 15 (2008).

[162] I. F. Sbalzarini and P. Koumoutsakos, J. Struct. Biol. 151, 182 (2005).

[163] T. J. Arbour and J. Enderlein, Lab Chip 10, 1286 (2010).

[164] H. Yokoyama, Mol. Cryst. Liq. Cryst. 165, 265 (1988).

[165] O. Yaroshchuk and Y. Reznikov, J. Mater. Chem. 22, 286 (2012).

[166] A. Rastegar and M. Škarabot, B. Blij, and Th. Rasing, J. Appl. Phys. 89, 960 (2001).

[167] Th. Rasing and I. Muševic, Surfaces and Interfaces of Liquid Crystals, Springer-Verlag Berlin Heidelberg, 2004.

[168] A. D. Price and D. K. Schwartz, Langmuir 22, 9753 (2006).

[169] I. Gerus, A. Glushchenko, SB. Kwon, V. Reshetnyak, and Y. Reznikov, Liq. Cryst. 28, 1709 (2001).

[170] J. Kim, M. K. Chaudhury, M. J. Owen and T. Orbeck, J. Coll. Int. Sci. 244, 200 (2001).

[171] J. C. McDonald, D. C. Duffy, J. R. Anderson, D. T. Chlu, H. Wu, O. J. A. Schueller, and G. M. Whitesides, Electrophoresis 21, 27 (2000).

[172] L. Yu, C. M. Li, Q. Zhou, and J. H. Luong, Bioconjug. Chem. 18, 281 (2007). 
[173] I. Gvozdovskyy, Y. Kurioz, and Y. Reznikov, Opt. Elect. Rev. 17, 116 (2009).

[174] B. Kim, E. T. K. Peterson, and I. Papautsky, Proc. IEEE Eng. Med. Biol. Soc. 7, 5013 (2004).

[175] T. R. N. Kutty and A. D. Fischer, Mol. Cryst. Liq. Cryst. 99, 301 (1983).

[176] J. M. Geary, J. W. Goodby, A. R. Kmetz and, J. S. Patel, J. Appl. Phys. 62, 4100 (1987).

[177] M. P. Valignat, S. Villette, J. Li, R. Barberi, R. Bartolino, E. Dubois-Violette, and A. M. Cazabat, Phys. Rev. Lett. 77, 1994 (1996).

[178] J. Ignés-Mullol, J. Baudry, P. Oswald, Phys. Rev. E 63, 031701 (2001).

[179] T. Ohzono and J. Fukuda, Nature Comm. 3, 701 (2012).

[180] J.-H. Kim, M. Yoneya, and H. Yokoyama, Nature 420, 159 (2002).

[181] A. de Lózar, W. Schöpf, I. Rehberg, D. Svensek, and L. Kramer, Phys. Rev. E 72, 051713 ( 2005).

[182] G. S. Ranganath, J. Phys. 40, Colloq. C3, 87 (1979).

[183] A. M. J. Spruijt, Solid St. Comm. 13, 1919 (1973).

[184] P.E. Cladis, W. van Saarloos, P. L. Finn, and A. R. Kortan, Phys. Rev. Lett. 58, 222 (1987).

[185] R. Holyst and P. Oswald, Phys. Rev. E 65, 041711 (2002).

[186] P. Tadapatri and K. S. Krishnamurthy, J. Phys. Chem. B 112, 13509 (2008).

[187] C. Williams and Y. Bouligand, J. Phys. (Paris) 35, 589 (1974).

[188] G. de Luca and A. D. Rey, J. Chem. Phys. 127, 104902 (2007).

[189] Y. Yi, J. E. Maclennan, and N. A. Clark, Phys. Rev. E 83, 051708 (2011).

[190] V. Tomar, S. I. Hernández, N. L. Abbott, J. P. Hernández-Ortiz, and J. J. de Pablo, Soft Matter 8, 8679 (2012). 
[191] H. Matthias, S. L. Schweizer, R. B. Wehrspohn, and H. S. Kitzerow, J. Opt. A 9, S389 (2007).

[192] T. Araki, M. Buscaglia, T. Bellini, and H. Tanaka, Nature Mater. 10, 303 (2011).

[193] T. Araki, private communication.

[194] R. Ondris-Crawford, E. P. Boyko, B. G. Wagner, J. H. Erdmann, S. Žumer, and J. W. Doane, J. Appl. Phys. 69, 6380 (1991).

[195] M. Ravnik, private communication.

[196] C. M. Dafermos, Quart. J. Mech. Appl. Math. 23, 549 (1970).

[197] D. R. Meldrum and M. R. Holl, Science 297, 1197 (2002).

[198] J. Glückstad, Nature Mater. 3, 9 (2004).

[199] A. B. Subramaniam, M. Abkarian, and H. A. Stone, Nature Mater. 4, 553 (2005).

[200] A. Terray, J. Oakey, and D. W. M. Marl, Science 296, 1841 (2002).

[201] H. Bruus, Theoretical Microfluidics, Oxford University Press, Oxford, 2008.

[202] A. D. Stroock, S. K. W. Dertinger, A. Ajdari, I. Mezic, H. A. Stone, and G. M. Whitesides, Science 295, 647 (2002).

[203] J. C. Baret, O. J. Miller, V. Taly, M. Ryckelynck, A. El-Harrak, L. Frenz, C. Rick, M. L. Samuels, J. B. Hutchison, J. J. Agresti, D. R. Link, D. A. Weitz, and A. D. Griffiths, Lab Chip 9, 1850 (2009).

[204] S. E. Chung, W. Park, S. Shin, S. A. Lee, and S. Kwon, Nature Mater. 7, 587 (2008).

[205] P. Abbyad, R. Dangla, A. Alexandrou, and C. N. Baroud, Lab Chip 11, 813 (2011).

[206] A. A. Sonin, A. Yethiraj, J. Bechhoefer, and B. J. Frisken, Phys. Rev. E 52, 6260 (1995).

[207] Ch. Gähwiller, Phys. Rev. Lett. 28, 1554 (1972).

[208] W. Helfrich, J. Chem. Phys. 50, 100 (1969).

[209] W. Helfrich, J. Chem. Phys. 56, 3187 (1972). 
[210] J. Frenkel, Kinetic Theory of Liquids , Dover, New York,1955.

[211] K. Negita, J. Chem. Phys. 105, 17 (1996).

[212] G. Toulouse and M. Kleman, J. Phys. Lett. 37, L149 (1976).

[213] V. P. Mineyev and G. E. Volovik, Phys. Rev. B: Solid State 18, 3197 (1978).

[214] O. D. Lavrentovich and S. S. Rozhkov, Pis'ma. Zh. Eksp. Teor. Fiz. 47, 210 (1988).

[215] D. R. Link, M. Nakata, Y. Takanishi, K. Ishikawa and H. Takezoe, Phys. Rev. Lett. 87, 195507 (2001).

[216] S. Zhang, E. M. Terentjev, and A. M. Donald, J. Phys. Chem. B 109, 13195 (2005).

[217] A. B. Nych, D. Y. Reznikov, O. P. Boiko, V. G. Nazarenko, V. M. Pergamenshchik, and P. Bos, Eur. Phys. Lett. 81, 16001 (2008).

[218] D. Psaltis, S. R. Quake, and C. Yang, Nature 442, 381 (2006).

[219] J. Leach, H. Mushfique, R. di Leonardo, M. Padgett, and J. Cooper, Lab Chip 6, 735 (2006)

[220] T. Pfohl, F. Mugele, R. Seemann, and S. Herminghaus, Chem. Phys. Chem. 4, 1291 (2003).

[221] P. Nghe, E. Terriac, M. Schneider, Z. Z. Li, M. Cloitre, B. Abecassis, and P. Tabeling, Lab Chip 11, 788 (2011).

[222] J. Feng and L. G. Leal, Phys. Fluids 11, 28121 (1999).

[223] C. Denniston, E. Orlandini, and J. M. Yeomans, Comput. Theor. Polym. Sci. 11, 389 (2001).

[224] C. Denniston, D. Marenduzzo, E. Orlandini, and J. M. Yeomans, Phil. Trans. R. Soc. Lond. A 362, 1745 (2004).

[225] J. Quintas Carou, B. R. Duffy, N. J. Mottram, and L. G. Leal, Phys. Fluids 18, 027105 (2006). 
[226] J. P. Hernandez-Ortiz, B. T. Gettelfinger, J. Moreno-Razo, and J. J. de Pablo, J. Chem. Phys. 134, 134905 (2011).

[227] L. Giomi, L. Mahadevan, B. Chakraborty, and M. F. Hagan, Phys. Rev. Lett. 106, 218101 (2011).

[228] L. Giomi and M. C. Marchetti, Soft Matter 8, 129 (2012).

[229] O. D. Lavrentovich, I. Lazo, and O. P. Pishnyak, Nature 467, 947 (2010).

[230] O. P. Pishnyak, S. V. Shiyanovskii, and O. D. Lavrentovich, Phys. Rev. Lett. 106, 047801 (2011).

[231] J. S. Lintuvuori, K. Stratford, M. E. Cates, and D. Marenduzzo, Phys. Rev. Lett. 105, 178302 (2010).

[232] Y.-J. Na, T-Y Yoon, S. Park, B. Lee, and S-D Lee, Chem. Phys. Chem. 11, 101 (2010).

[233] Z. Guo, C. Zheng, and B. Shi, Phys. Rev. E 65, 046308 (2002).

[234] H. Heuer, H. Kneppe, and F. Schneider, Mol. Cryst. Liq. Cryst. 214, 43 (1992).

[235] S. Chono and T. Tsuji, Mol. Cryst. Liq. Cryst. 309, 217 (1998).

[236] T. Araki and H. Tanaka, J. Phys.: Condens. Matter 18, 193 (2006).

[237] H. Stark and D. Ventzki, Phys. Rev. E 64, 031711 (2001).

[238] A. S. Utada, A. Fernandez-Nieves, H. A. Stone, and D. A. Weitz, Phys. Rev. Lett. 99, 094502 (2007).

[239] A. Rapini, L. Léger, and A. Martinet, J. Phys. Colloques 36, C1-189 (1975).

[240] R. Turner, Phil. Mag. 30, 13 (1974).

[241] H. Imura and K. Okano, Phys. Lett. A 42, 403 (1973).

[242] S. H. Lee, S-E Choi, A. J. Heinz, W. Park, S. Han, Y. Jung, and S. Kwon, Small 6, 2668 (2010). 


\section{Acknowledgements}

The work has been financially supported by the EC Marie Curie ITN project Hierarchy (PITN-CA-2008-215851) and the Max Planck Gesellschaft.

As I started my doctoral research in the summer of 2009, I was excited, enthused, and apprehensive. Excited that I had entered the hallowed portals of the 'Max-Planck-Institüt für Dynamik und Selbstorganization' (MPIDS); enthused, since I was formally stepping into the realms of Physics; and apprehensive, as I was embarking upon a research direction, which till then lacked any systematic investigation.

Thus, my first thanks are due to Dr. Christian Bahr, who as an advisor exuded generous conviction and trust, which helped me to think independently. Sincere thanks to Christian for introducing me to the colourful and marvelous world of liquid crystals. Christian's timely scientific inputs have contributed significantly in making my doctoral stint a highly enriching one. He has been ever-ready in any and every kind of support I ever required.

I would like to express my sincere gratitude to Prof. Dr. Stephan Herminghaus. My proximity to Stephan during the final stages of my research has left an indelible mark on my general philosophy of life and work. In spite of his professional obligations, Stephan always had time for me: be it even on a sunny spring Sunday. I consider myself to be rather privileged to have received so much attention from him. Furthermore, the overall ambiance that he has successfully managed to instill within our department of Dynamics of Complex Fluids $(D C F)$, has contributed not only to our professional skills, but also to our personal demeanour. Stephan's direct and indirect contributions to my overall upbringing in Göttingen can hardly be overemphasized.

And when I mention Stephan, can Monika be far away? The day I arrived in Göttingen to hold my interview-talk at the Bunsenstraße, I felt perfectly at home. My heartfelt thanks to Monika Teuteberg for making that partly possible. Over the years, Monika has incessantly 
helped make my stay in Göttingen smooth and easy, at times even without my knowledge. $D C F$ without Monika is incomprehensible.

I am thankful to Prof. Dr. Jörg Enderlein for his consent to be a member of the doctoral thesis committee. I was fortunate to collaborate with his group, especially with Christoph Pieper, during my thesis. Prof. Enderlein's constant encouragement and eagerness in the present work is highly appreciated. My thanks are also due to Prof. Dr. Julia Yeomans (Oxford) and Dr. Miha Ravnik (Oxford/Ljubljana) for providing me with the much needed numerical support. I thank Dr. Elena Ouskova (Aalto, Finland) for introducing me to the field of photo-induced reorientation in liquid crystals and providing the light-sensitive samples for my experiments.

I wish to express my thanks to all my colleagues and visiting scholars at the MPIDS, who have supported me in different capacities at different points of this doctoral thesis. Particularly, I thank Prof. Dr. Thomas Pfohl (Basel), Prof. Dr. Jürgen Vollmer, Dr. Jean-Christophe Baret, Dr. Björn Hof, Dr. Matthias Schröter, Dr. Martin Brinkmann, Dr. Marco G Mazza, Prof. Dr. Ralf Stannarius (Magdeburg), Prof. Dr. Karen E Daniels (North Carolina), and Prof. Dr. Clemens Bechinger (Stuttgart). I extend my thanks also to Sibylle Nägle, Thomas Eggers (and his sense of humour !), Markus Benderoth, Wolf Keiderling and Udo Krafft for providing the key support systems on which this research could be conducted. Special thanks to the members of the Liquid Crystal group, and to all my colleagues from DCF, MPIDS. And of course, to $M r$. Trümper for the rides to the university campus and the wonderful discussions en route.

I am thankful to the Bandol Summer School on Liquid Crystals-2009 for broadening my view on liquid cystals. My special thanks also to Prof. Dr. Igor Muševič, Prof. Dr. Slobodan Žumer, Dr. Miha Škarabot, Dr. Andriy Nych, Dr. Miha Ravnik, Dr. Matjaž Humar, Jampani V. S. Rao, and especially to Dr. Uroš Tkaleč for making my trips to Slovenia not only productive, but highly enjoyable. My thanks also goes to Prof. Dr. Franz Durst and Susanne Braun for the support during my intial phase in Erlangen, and to Prof. Dr. Suman Chakraborty for being the wonderful friend and philosopher to me.

It is an incomplete chapter if I fail to mention the people who took due care of my life beyond the scientific boundaries. Though words shall fail to convey my feelings, I am grateful to all my friends for making my stay in Göttingen so very special. Especially, thanks to Ben for enlightening me that weekends are normally not for working; to Paul for showing me that life has more to it than what appears; to Eric for being the hard nut with indeed a soft-within; to Kris for being perfectly normal in spite of his tremendous looks; to Quentin for making me a part of his finesse; to Anne for all her strength; to Marta for filling me up with positive energy everytime we met; to Ciro for the games he taught me; to Mihaela for her company; 
to Domi and Antoinne for joining in, though a little belated; and to Ivi and Alex for making the half-yearly Marie Curie meetings a little more eventful.

My heartfelt thanks to Melanie for her never-ending support, cooperation and patience. I am grateful to her for blending me into the life and culture of this country which has now become a part of my being. I owe her all the vacations which were either postponed (indefinitely) or cancelled in pursuance of this work.

Last, but certainly far from being the least, I am indebted to my mother, father and brother for their blessings and good wishes. Had it not been for their sacrifices, often at the expense of their own comfort, this day would have been far from reality. The strength and ability to dream, and to work towards realizing them, are solely due to the upbringing I had since my childhood. Each little step that I take forward makes me only a humbler man.

Anupam Sengupta

Göttingen

October 2012 



\section{Erklärung}

1. Ich habe diese Arbeit selbständig und ohne unerlaubte Hilfe angefertigt und dabei nur die angegebenen Quellen benutzt.

2. Eine entsprechende Promotion wurde an keiner anderen Hochschule im In- oder Ausland beantragt; die eingereichte Dissertation oder Teile von ihr wurden nicht für ein anderes Promotionsvorhaben verwendet.

Göttingen, den 31 Oktober 2012.

Anupam Sengupta 



\section{Scientific Papers Published :}

(1) A. Sengupta, U. Tkalec, and C. Bahr, 'Nematic textures in microfluidic environment', Soft Matter, 7, 6542 (2011).

(2) A. Sengupta, S. Herminghaus, and C. Bahr, 'Nematic liquid crystals and nematic colloids in microfluidic environment', Mol. Cryst. Liq. Cryst., 547, 203 (2011).

(3) A. Sengupta, B. Schulz, E. Ouskova, and C. Bahr, 'Functionalization of microfluidic devices for investigation of liquid crystal flows', Microfluidics and Nanofluidics, 13, 941, (2012).

(4) A. Sengupta, S. Herminghaus, and C. Bahr, 'Opto-fluidic velocimetry using liquid crystal microfluidics', Appl. Phys. Lett., 101, 164101 (2012).

(5) A. Sengupta, U. Tkalec, M. Ravnik, J. Yeomans, C. Bahr, and S. Herminghaus, 'Liquid crystal microfluidics for tunable flow shaping', Phys. Rev. Lett., 110, 048303 (2013).

(6) A. Sengupta, C. Pieper, C. Bahr, J. Enderlein, and S. Herminghaus, 'Flow of a nematogen past a micro-pillar', Soft Matter, 9, 1937 (2013).

(7) A. Sengupta, C. Bahr, and S. Herminghaus, 'Topological microfluidics for flexible microcargo concepts', Nat. Commun. (under review).

(8) A. Sengupta, S. Herminghaus, and C. Bahr, 'Spatio-temporal modulation of nematic micro-flows', 2012 (in preparation).

(9) A. Sengupta, S. Herminghaus, and C. Bahr, 'Microfluidics of liquid crystals and their dispersions', Liquid Crystal Reviews, 2012 (invited review article, in preparation). 



\section{Invited and contributed talks :}

Invited Talks:

1. Max Planck Institute Campus Seminar Series, Göttingen, 2012-2013 (12.09.2012)

Title: Topological soft rails for transport of microfluidic cargo

2. 24 $^{\text {th }}$ International Liquid Crystal Conference, Mainz (20.08.2012)

Title: Nemato-microfluidics: Interplay between flow, confinement and surface anchoring on a microfluidic platform

3. 76 $^{\text {th }}$ German Physical Society Spring Meeting, Berlin (30.03.2012)

Title: Guided and spatio-selective transport via anisotropic fluid flows

4. Invited seminars at the Department of Physics, University of Stuttgart and Faculty of Physics, University of Ljubljana (2012)

Selected Conference Presentations:

1. American Chemical Society: Colloids and Surface Science Symposium, Baltimore, June, 2012

2. 39 $^{\text {th }}$ German Liquid Crystal Conference, Hamburg, March, 2011

3. 75 $^{\text {th }}$ German Physical Society Spring Meeting, Dresden, March, 2011

4. 23 $^{\text {rd }}$ International Liquid Crystal Conference, Krakow, July, 2010

5. Gordon Research Conference on Liquid Crystals, Mount Holyoke, June, 2011 (Poster presentation) 



\section{Curriculum Vitae}

\section{Personal Details}

Name : $\quad$ Sengupta Anupam

Date of birth : $\quad 31.05 .1983$

Place of birth : Burnpur, West Bengal, India

Address : $\quad$ Christophorusweg 12, 37075 Göttingen

Nationality : Indian

\section{Education}

\begin{tabular}{|c|c|}
\hline $06 / 2009-10 / 2012$ & $\begin{array}{l}\text { Doctoral research at the Max Planck Institute for Dynamics } \\
\text { and Self Organization, Göttingen, Germany }\end{array}$ \\
\hline & Supervisors: Dr. Christian Bahr and \\
\hline & Prof. Dr. Stephan Herminghaus \\
\hline $2008-2009$ & Application Engineer, FMP Technology GmbH, \\
\hline & Erlangen, Germany \\
\hline & Supervisor: Prof. Dr. Franz Durst \\
\hline $2003-2008$ & Bachelor of Technology in Mechanical Engineering and \\
\hline & Master of Technology in Thermal and Fluids Engineering, \\
\hline & Indian Institute of Technology, Bombay, India \\
\hline & Supervisor: Prof. A. W. Date \\
\hline
\end{tabular}

